University of Louisville ThinkIR: The University of Louisville's Institutional Repository

Electronic Theses and Dissertations

$5-2016$

\title{
A comparison of younger and older couples coping with breast cancer : examining the relationship among mutuality, dyadic coping, and quality of life of patients and partners.
}

Chiara Acquati

University of Louisville

Follow this and additional works at: https://ir.library.louisville.edu/etd

Part of the Social Work Commons

\section{Recommended Citation}

Acquati, Chiara, "A comparison of younger and older couples coping with breast cancer : examining the relationship among mutuality, dyadic coping, and quality of life of patients and partners." (2016). Electronic Theses and Dissertations. Paper 2392.

https://doi.org/10.18297/etd/2392

This Doctoral Dissertation is brought to you for free and open access by ThinkIR: The University of Louisville's Institutional Repository. It has been accepted for inclusion in Electronic Theses and Dissertations by an authorized administrator of ThinkIR: The University of Louisville's Institutional Repository. This title appears here courtesy of the author, who has retained all other copyrights. For more information, please contact thinkir@louisville.edu. 
A COMPARISON OF YOUNGER

AND OLDER COUPLES COPING WITH BREAST CANCER:

EXAMINING THE RELATIONSHIP AMONG MUTUALITY, DYADIC COPING, AND QUALITY OF LIFE OF PATIENTS AND PARTNERS

By

Chiara Acquati

B.A. Psychology, Catholic University of the Sacred Heart, Milan, 2005

M.A. Clinical Psychology, Catholic University of the Sacred Heart, Milan, 2007

M.S.W., Boston College, 2011

A Dissertation

Submitted to the Faculty of the

Kent School of Social Work of the University of Louisville

In Partial Fulfillment of the Requirements

For the Degree of

Doctor of Philosophy

in Social Work

Kent School of Social Work

University of Louisville

Louisville, Kentucky

And

College of Social Work

University of Kentucky

Lexington, Kentucky

May 2016 
Copyright 2016 by Chiara Acquati

All rights reserved 

A COMPARISON OF YOUNGER

AND OLDER COUPLES COPING WITH BREAST CANCER:

EXAMINING THE RELATIONSHIP AMONG MUTUALITY, DYADIC COPING, AND QUALITY OF LIFE OF PATIENTS AND PARTNERS

\section{By}

\section{Chiara Acquati}

B.A. Psychology, Catholic University of the Sacred Heart, Milan, 2005

M.A. Clinical Psychology, Catholic University of the Sacred Heart, Milan, 2007 M.S.W., Boston College, 2011

A Dissertation Approved on

April 6, 2016

By the following Dissertation Committee:

Dr. Karen Kayser, Chair

Dr. Wanda Lott Collins

Dr. Eli Karam

Dr. Kristen Mark

Dr. Heehyul Moon 


\section{DEDICATION}

This dissertation is dedicated to my husband,

Lorenzo Alessandro Stermieri, and to my daughter,

Ginevra Alessandra Acquati Stermieri.

Everything I hold dear in my life you have given me. 


\section{ACKNOWLEDGEMENTS}

This dissertation represents the culmination of the work conducted during the years of the doctoral program and my previous research experiences in psychosocial oncology. I owe my deepest gratitude to many who have provided ongoing support, guidance and encouragement.

First and foremost I offer my sincerest gratitude to my mentor and my supervisor, Dr. Karen Kayser. You have provided me with countless learning opportunities, participating in research projects, developing courses, and collaborating on publications and presentations. Over the years your modeling of scholarly social work research, teaching and service to the profession has had a tremendous impact on me, guiding and shaping my professional development and my desire to continue to serve and advocate for those affected by cancer for many years to come.

I would also like to thank the other committee members for their support, encouragement and very attentive comments. Dr. Heehyul Moon and Dr. Kristen Mark, you have been my continuous reference for methodology and data analysis questions, offering numerous insights and suggestions about the best strategies to analyze this dataset and the implications of this work. You have been also great role models of young successful women in academia. Dr. Wanda Lott Collins, since our first class together you have been an inspiration for your ability to be a passionate and kind teacher, and a very 
rigorous researcher. You have always found the right words to support me and you have always believed in me. I am very grateful. Dr. Eli Karam, working with you on this project and other research endeavors, I have always admired your calming and supportive presence, and your ability to provide meaningful insights and comments.

Many funders helped make my doctoral training and research possible. My highest gratitude goes to The American Cancer Society for awarding me with three years of funding. Through this experience I have had the opportunity to attend conferences, institutes, and to work with the brightest minds in our field. These experiences have been instrumental for my professional development. As part of the grant I was able to work with Dr. Deborah Kashy. I will always cherish the conversations we had about methodology and data analysis. You have provided me with a powerful lesson: that my understanding of relationships and dyadic data analysis is a long-term process, and that this research project was just the first step. The University of Louisville's School of Interdisciplinary and Graduate Studies, the Kent School of Social Work, supported me tremendously as well. My whole-hearted thank you to the faculty and to the members of my cohort.

A very special thank you goes to Dr. Emanuela Saita. It was under her tutelage that I became interested in couples and cancer when completing my first master's degree. Over the years she became a mentor and a friend, sharing advices and suggestions about my academic career, personal life, and everything else.

Words cannot describe the gratitude and love I feel for my parents. They have always valued my education above all, making huge sacrifices to make it possible for me to attend the best schools and to benefit from every opportunity. They have been the 
foundation of my life and shared my enthusiasm for the decision to pursue a doctoral program abroad, even if it meant I would have been away from them.

To my husband Lorenzo, for the love, dedication and support you have given me through these years. This wasn't your aspiration, yet you have moved to another country to allow me to pursue my dream and to do what I love. These years have been extremely challenging for us, but our relationship has grown and has been strengthen during this time. I look forward to see where our journey together will take us. 


\begin{abstract}
A COMPARISON OF YOUNGER

AND OLDER COUPLES COPING WITH BREAST CANCER: EXAMINING THE RELATIONSHIP AMONG MUTUALITY, DYADIC COPING, AND QUALITY OF LIFE OF PATIENTS AND PARTNERS
\end{abstract}

\title{
Chiara Acquati
}

April 6, 2016

Background Despite the extensive literature available about the psychosocial adaptation to breast cancer, the experience of younger women and their partners has been mostly neglected by the research. While younger couples have been identified at higher risk because of less collaborative behaviors, higher vulnerability to distress, and poorer quality of life, in most contributions couples are treated as a homogeneous group. This study was then designed to examine the differential impact of the illness on younger dyads, by comparing them to a group of older couples. A significant gap in the literature about dyadic coping was also addressed, which is the understanding of how dyadic coping originates and it is associated with higher quality of life.

Methods Participants have been recruited among newly diagnosed women with earlystage breast cancer, for a total of 86 couples. Patients below the age of 45 and their partners were considered younger couples and compared to the remaining 51 older couples. The Actor Partner Interdependence Model (APIM) was used to examine actor 
and partner effects of mutuality on dyadic coping. A simple mediation model tested whether dyadic coping mediates the relationship between mutuality and quality of life. Summary of Findings Younger women reported significantly worse quality of life, with worse functioning in physical, social and emotional domains than older patients. Similarly, younger partners experienced higher illness intrusiveness, worse emotional well-being, and maladaptive dyadic coping. Younger couples' positive and negative coping styles were the result of both actor and partner effects of mutuality, indicating higher interdependence in their relationship. Finally, negative dyadic coping mediated the relationship between mutuality and quality of life for younger patients and older partners. Conclusions Younger couples' adaptation to breast cancer is significantly compromised not only because of the more negative impact of the illness on the individual's wellbeing, but also as a result of higher reciprocal influence of mutuality in predicting both adaptive and maladaptive dyadic coping behaviors. Future studies should continue to examine the developmental trajectory of dyadic coping over the life-span and psychosocial interventions should be developed to promote positive adaptation to cancer in a time of higher vulnerability. 


\section{TABLE OF CONTENTS}

DEDICATION

PAGE

ACKNOWLEDGEMENTS

iii

ABSTRACT

LIST OF TABLES

LIST OF FIGURES

iv

vii

xii

$\mathrm{XV}$

\section{CHAPTER I: INTRODUCTION TO PROBLEM}

Coping With Breast Cancer at a Younger Age

- Quality of Life and Psychological Well-being 4

- Body Image and Sexual Functioning

- Impact of Breast Cancer on the Couple Relationship of Younger Patients

Cancer as a Relational Illness: The Interdependence of Patients and Partners

The Impact of Cancer on the Couple Relationship

- Quality of Life and Psychological Well-being

- Communication

- Sexuality and Intimacy

- Adjustment and Benefit Finding

\section{CHAPTER II: LITERATURE REVIEW}

Dyadic Coping and its Relevance for the Marital Relationship in a Time of Crisis

- Relationship-focused coping

- Congruence Model

- Systemic-Transactional Model

- Relational-Cultural Model

- Developmental-Contextual Model

Toward a Conceptualization of Dyadic Coping to study Couples' Adjustment to Cancer

- Conceptual Framework

- Theoretical Framework 
ii. $\quad$ Transactional Theory of Stress and Coping 54

iii. $\quad$ Relational-Cultural Theory 59

iv. Life Course Developmental Framework 63

Summary

\section{CHAPTER III: METHODOLOGY}

Research Goals and Hypotheses

- $\quad$ Specific Aim 1

- $\quad$ Specific Aim 2

- $\quad$ Specific Aim 3

- $\quad$ Specific Aim 4

Research Design

Data Source and Sampling

Operationalization of Variables

Statistical Analysis

- $\quad$ Specific Aim 1

- $\quad$ Specific Aim 2

90

- $\quad$ Specific Aim 3

91

- $\quad$ Specific Aim 4

95

Summary

\section{CHAPTER IV: RESULTS}

Data Preparation and Preliminary Data Analysis 102

Description of Sample

- Socio-Demographics, Relational and Clinical Characteristics

- Variables of Interest

- Younger and Older Couples

Correlations

- Bivariate Analyses: Patients

- Bivariate Analyses: Patients

Specific Aim 1

Specific Aim 2

\section{CHAPTER V: DISCUSSION}

Discussion

Limitations

Future Directions

Conclusion 
APPENDIX A

Survey

332

IRB Approval Letter

354

APPENDIX B

Bivariate Analyses: Patients

355

Bivariate Analyses Partners

360

APIM Calculations

364

CURRICULUM VITAE

368 


\section{LIST OF TABLES}

TABLE

PAGE

1. Overview of Dyadic Coping Models 27

2. Description of Positive and Negative Dyadic Coping Styles 35

3. Operationalization of Variables for Patients and Partners 87

4. Score calculation and Reliability of Instruments 88

5. Statistical Analysis Overview 97

6. Socio-Demographic, Relational and Clinical Characteristics of the 105 Sample

7. Sample Characteristics of the Major Study Variables 108

8. Socio-Demographic, Relational and Clinical Characteristics of 113 Younger Couples

9. Sample Characteristics of the Major Study Variables for Younger 115 Couples

10. Socio-Demographic, Relational and Clinical Characteristics of Older Couples

11. Sample Characteristics of the Major Study Variables for Older Couples

12. Independent Samples t-Test comparing Dyadic Coping, Mutuality, Communication, and Quality of Life among Younger and Older breast cancer patients

13. Independent Samples t-Test comparing Dyadic Coping, Mutuality, Communication, and Quality of Life among Younger and Older Partners

14. Bivariate Correlations of Quality of Life Scores of Younger Patients and Partners

15. Bivariate Correlations of Quality of Life Scores of Older Patients and Partners

16. Bivariate Correlations of Dyadic Coping Scores of Younger

Patients and Partners 
17. Bivariate Correlations of Dyadic Coping Scores of Older Patients and Partners

18. Bivariate Correlations for Younger Breast Cancer Patients Scores on Quality of Life and Dyadic Coping

19. Bivariate Correlations for Younger Partners Scores on Quality of Life and Dyadic Coping

20. Multiple Regression Analysis Predicting Social Well-being Score for Breast Cancer Patients

21. Multiple Regression Analysis Predicting Emotional Well-being Score for Breast Cancer Patients

22. Multiple Regression Analysis Predicting FACT-G Score for Breast Cancer Patients

23. Multiple Regression Analysis Predicting Breast Cancer Symptoms Score for Breast Cancer Patients

24. Multiple Regression Analysis Predicting FACT-B Score of Breast Cancer Patients

25. Multiple Regression Analysis Predicting Emotional Well-being of Partners

26. Multiple Regression Analysis Predicting Illness Intrusiveness of Partners

27. Multilevel regression coefficients estimating actor and partner effects of Mutuality on Stress Communication

28. Multilevel regression coefficients estimating actor and partner effects of Mutuality on Common Dyadic Coping

29. Multilevel regression coefficients estimating actor and partner effects of Mutuality on Positive Dyadic Coping

30. Simple slope regression coefficients estimating separate Mutuality actor and partner effects for patients and caregivers on Positive Dyadic Coping

31. Multilevel regression coefficients estimating actor and partner 162 effects of Mutuality on Hostile Dyadic Coping

32. Simple slope regression coefficients estimating separate mutuality 162 actor and partner effects for patients and caregivers on Hostile Dyadic Coping

33. Multilevel regression coefficients estimating actor and partner effects of Mutuality on Avoidance of Dyadic Coping

34. Multilevel regression coefficients estimating actor and partner effects of Mutuality on Stress Communication of Younger Couples 
35. Multilevel regression coefficients estimating actor and partner effects of Mutuality on Stress Communication in Older Couples

36. Multilevel regression coefficients estimating Actor and Partner effects of Mutuality on Common Dyadic Coping scores of Younger Couples

37. Multilevel regression coefficients estimating actor and partner effects of Mutuality on Common Dyadic Coping in Older Couples

38. Multilevel regression coefficients estimating actor and partner effects of Mutuality on Positive Dyadic Coping in Younger Couples

39. Multilevel regression coefficients estimating actor and partner

175 effects of Mutuality on Positive Dyadic Coping in Older Couples

40. Simple slope regression coefficients estimating separate mutuality actor and partner effects for patients and caregivers on Positive Dyadic Coping in Older Couples

41. Multilevel regression coefficients estimating Actor and Partner effects of Mutuality on Hostile Dyadic Coping in Younger Couples

42. Simple slope regression coefficients estimating separate mutuality actor and partner effects for patients and caregivers on Hostile Dyadic Coping in Younger Couples

43. Multilevel regression coefficients estimating actor and partner effects of Mutuality on Hostile Dyadic Coping in Older Couples

44. Multilevel regression coefficients estimating Actor and Partner effects of Mutuality on Avoidance of Dyadic Coping in Younger Couples

45. Multilevel regression coefficients estimating actor and partner effects of Mutuality on Avoidance of Dyadic Coping in Older Couples

46. Model coefficients of the Simple Mediation Model between

Mutuality, Avoidance of Dyadic Coping and Social Well-being for Younger Patients

47. Model coefficients of the Simple Mediation Model between Mutuality, Hostile Dyadic Coping and Illness Intrusiveness for Older Partners 


\section{LIST OF FIGURES}

1. Overview of Positive and Negative Forms of Dyadic Coping 35

2. Relational-Cultural Coping Process 43

3. Conceptual Framework of the Study 52

4. $\quad$ Proposed APIM Model of Mutuality and Dyadic Coping 99

5. Conceptual Diagram of the proposed Simple Mediation Model with 99 Dyadic Coping mediating the relationship between Mutuality and Quality of Life of Patients and Partners

6. The actor and partner effects of Mutuality as predictors of Stress Communication

7. The actor and partner effects of Mutuality as predictors of Common Dyadic Coping

8. The actor and partner effects of Mutuality as predictors of Positive Dyadic Coping

9. The actor and partner effects of Mutuality as predictors of Hostile Dyadic Coping

10. The actor and partner effects of Mutuality as predictors of Avoidance of Dyadic Coping.

11. The actor and partner effects of Mutuality as predictors of Stress Communication in Younger Couples

12. The actor and partner effects of Mutuality as predictors of Stress Communication in Older Couples

13. The actor and partner effects of Mutuality as predictors of Common Dyadic Coping in Younger Couples

14. The actor and partner effects of Mutuality as predictors of Common Dyadic Coping in Older Couples

15. The actor and partner effects of Mutuality as predictors of Positive Dyadic Coping in Younger Couples 
16. The actor and partner effects of Mutuality as predictors of Positive Dyadic Coping in Older Couples

17. The actor and partner effects of Mutuality as predictors of Hostile Dyadic Coping in Younger Couples

18. The actor and partner effects of Mutuality as predictors of Hostile Dyadic Coping in Older Couples

19. The actor and partner effects of Mutuality as predictors of Avoidance of Dyadic Coping in Younger Couples

20. The actor and partner effects of Mutuality as predictors of Avoidance of Dyadic Coping in Older Couples

21. Statistical diagram of the Simple Mediation Model 185

22. Simple Mediation Model for Younger Patients

186

23. Simple Mediation Model for Older Partners

191 


\section{CHAPTER I: INTRODUCTION TO PROBLEM}

The chapter presents the experience of women coping with breast cancer at a younger age and the unique impact the illness has on quality of life, psychological distress, body image, sexuality and close relationships. The limited literature addressing the experience of younger couples coping with breast cancer is reviewed in detail. Then, the chapter offers an overview of the psychosocial consequences of cancer on the couple relationship, with a specific focus on quality of life, communication, sexual functioning and benefit finding to support the significance of assuming a relational perspective when addressing couples coping with cancer.

\section{Coping with Breast Cancer at a Younger Age}

The adaptation to a diagnosis of breast cancer continues to be one of the most studied areas of psychosocial oncology research (Rowland \& Massie, 2010). This is due in large measure to its prevalence, as recent data from the American Cancer Society indicate that it represents the most frequently diagnosed cancer in women, accounting for 29\% of all new female cancers (American Cancer Society, 2015: 2016; Siegel, Miller \& Jemal, 2015). But the current interest for breast cancer also reflects the fact that it involves an area of the body with great symbolic meaning for women and their partners, 
and it requires complex decision-making in terms of treatment and care. The ability of the individual to cope with breast cancer is influenced by the existing socio-cultural context, psychological characteristics of the woman, and clinical factors (Rowland \& Massie, 2010). More recently, the literature has given increasing attention to the quality of close relationships and the couple's coping abilities as essential resources to promote better adjustment (Kayser, Watson \& Andrade, 2007; Revenson, Kayser \& Bodenmann, 2005; Saita, 2009; Traa, DeVries, Bodenmann \& Den Oudsten, 2015b).

Although younger women constitute a minority among breast cancer patients, with approximately just $20 \%$ of new cases registered among women younger than the age of 50 (American Cancer Society, 2014), epidemiological and clinical indications isolate them from the rest. Not only breast cancer in younger women is associated to more aggressive biological characteristics such as higher-grade tumors and advanced stage of disease, but worse prognosis and higher relapse rate are common (Ademuyiwa et al., 2015; Beadle, Woodward, \& Buchholz, 2011). At the moment, in fact, breast cancer represents the leading cause of cancer death for women between 20 to 39 years of age in the USA, and the trend is maintained also when considering the group between 40 and 59 years of age (Siegel et al., 2015).

Younger women with breast cancer represent a distinct group of patients not only in vitue of medical characteristics, but especially because of the unique challenges and psychosocial needs they are confronted with ${ }^{1}$ (Armuand, Wettergren, RodriguezWallberg \& Lampic, 2014; Bell, Ziner \& Champion, 2009; Fergus et al., 2014; Gorman

\footnotetext{
${ }^{1}$ Since a clear definition of a "younger woman with cancer" is still elusive in the current literature, in the present chapter findings from articles addressing women with breast cancer from the early 30 s to the $50 \mathrm{~s}$ are presented as reflective of the experience of younger women.
} 
et al., 2011; Howard-Anderson, Ganz, Bower \& Stanton, 2012; Koch et al., 2013; Morrow et al., 2014). From a developmental perspective, younger women are in a stage of their lives when they are invested in self-realization and accomplishing social milestones. Those are the years when most women are married or involved in a committed relationship, having children and focusing on their professional careers and aspirations (Ahmad, Fergus, \& McCarthy, 2015; Fergus et al., 2015). The cancer diagnosis introduces not only an "out of time" confrontation with mortality, but it creates abrupt and premature changes in their quality of life. Younger women with breast cancer face reproductive concerns about fertility and childbearing, changes in their relationships with children and family members, multiple role demands, career concerns related to job security and financial difficulties, and greater concerns about body image and sexuality (Avis, Crawford, \& Manuel, 2004; Avis, Crawford, \& Manuel, 2005; Dunn \& Steginga, 2000; Ganz et al. 2003; Howard-Anderson et al., 2012; Ruddy et al., 2013). Breast cancer in younger women significantly affects the woman's family and social network, with concerns reported especially for the relationship with the partner and children (Ahmad et al., 2015; Baucom, Porter, Kirby, \& Gremore, 2005; Borstelmann et al., 2015; Ruddy et al., 2013).

As a consequence of these greater disruptions to developmental goals and tasks, several domains of functioning are extensively affected by breast cancer and its treatment. Often these effects can only be completely understood and witnessed over time. In the next paragraphs issues related to quality of life and psychological well-being, body image, sexual functioning and the relationship with the partner are reviewed. 


\section{Quality of Life and Psychological Well-being}

The literature has consistently revealed that younger women experience significantly higher levels of psychological distress and poorer quality of life compared to patients diagnosed later in life, with particularly worse emotional and social functioning registered both during active treatment and survivorship (Bantema-Joppe et al., 2015; Champion et al., 2014; Cataldo et al., 2013; Hartl et al, 2010; Hau et al., 2013; Luutonen et al., 2011; So et al., 2011).

A recent systematic review has demonstrated that younger women experience more difficulties adjusting to the cancer diagnosis because of its pervasive impact of numerous domains of quality of life (Howard-Anderson et al., 2012). Younger patients lament higher physical symptoms associated with the treatment and the perceived body disfigurement, like pain, lymphedema, sleep problems, and weight gain. They are also more vulnerable to the limitations caused by the illness to their social and intimate relationships, reporting higher concerns for their relationship with partners, difficulties disclosing the diagnosis, and higher feelings of isolation (Ahmad et al., 2015; Ruddy et al., 2013). Finally, younger women diagnosed with breast cancer present higher psychological symptoms, including higher anxiety, depression as well as persistent and intrusive worries that the cancer will return (Thewes et al., 2013). Worse physical wellbeing has been associated to younger age, absence of a significant relationship, and more aggressive treatment; while an increased risk for psychological difficulties has been identified among younger, unmarried, and low income patients (Antoine et al, 2008; Armer, 2005; Charif et al., 2015; Hopwood et al., 2007; Hoyer et al., 2011; Mertz et al., 2012; So et al., 2011, Tuinman, Van Nuenen, Hagedoorn, \& Hoekstra-Weebers, 2015). 
When compared to older survivors and age-matched controls, younger breast cancer survivors present more cognitive problems and fatigue (Champion et al., 2014). Longitudinal studies have highlighted functional declines in physical, social and psychological domains over time (Avis et al., 2004, Bloom et al., 2012; DiSipio, Hayes, Newman, \& Janda, 2008; Kroenke et al., 2004). Bloom et al. (2012) examined the persistence of problems at 5 and 10 years into survivorship. Again, younger women reported poorer general health, low physical well-being, reduced sexual activity and more chronic conditions. Younger survivors also experience greater psychological morbidity: higher level of depression, anxiety and fear of recurrence are consistently reported within five years post diagnosis (Champion et al., 2014; Crist \& Grunfield, 2013; Hopwood et al., 2007; Howard-Anderson et al., 2012; Ganz et al. 2003; Kroenke et al., 2004). In particular, anxiety and depressive symptoms have been found among those who had received chemotherapy and reported low level of support from closed ones and partners (Borstelmann et al., 2015; Gold et al., 2015). The co-occurrence of anxiety and depressive symptoms has a significant impact on the quality life of the individual, with lower levels of physical, psychological and social well-being documented for these women. Furthermore, researchers have noticed that anxiety symptoms influence the ability of the patient to adjust to the disease, leading to increased hopelessness, uncertainty, loss of control and low life satisfaction (Gold et al., 2015).

\section{Body Image and Sexual Functioning}

Body image and sexual difficulties acquire a significant meaning among younger women, as they often represent the most challenging consequences of treatment and the long term legacy of the illness on individual well-being and close relationships (Bantema- 
Joppe et al., 2015; Burwell et al., 2008; Champion et al., 2014; Fobair et al., 2006; Geue, Schmidt, Sender, Sauter, \& Friedrich, 2015; Hill et al., 2011; Jankowska, 2013).

The loss of a breast, surgical scarring, alopecia, weight changes and lymphedema negatively impact the body image of a younger woman. Furthermore, as treatment is often more aggressive than for older patients, younger women who undergo mastectomy and receive radiation present more numerous complaints compared to patients receiving breast-conserving surgery (Avis et al., 2005; Biglia et al., 2010; Paterson, Lengacher, Donovan, Kip, \& Tofthagen, 2016). However, the possibility of reconstruction mitigates the risk for body image concerns (Rosenberg et al., 2013) and seems to promote the ability to enjoy intimacy with a partner, as $80 \%$ of younger survivors who were not offered reconstruction continued to covered their body during intimate contacts (Andrzejczak, Markocka-Maczka, \& Lewandowski, 2013). The importance of body image for this group of patients has been confirmed by studies that examined its association with physical and psychological distress, impaired sexual functioning, and intimacy (Paterson et al., 2016). Very concerning is the fact that body image issues do not improve with time, with more negative indications in the treatment phase and again after one year (Bloom, Stewart, Chnag \& Banks, 2004; Biglia et al., 2010).

A negative body image has often been associated with greater sexual issues (Paterson et al., 2016). Sexual problems of younger breast cancer patients include vaginal dryness, reduced frequency of sexual activity and reduced breast sensitivity (Baucom et al., 2005; Carr, 2013; Cebeci, Yangin \& Tekeli, 2010). Treatments like surgery, radiation and chemotherapy can induce premature menopause, and reduced libido. Not only these symptoms directly affect sexual desire and comfort during intercourse, but the resulting 
fatigue can contribute to less interest in sexual activity (Avis et al., 2005; Bantema-Joppe et al., 2015; Baucom et al., 2005; Biglia et al., 2010; Champion et al., 2014; Fobair et al., 2006; Burwell et al., 2008; Jankowska, 2013). For example, a recent study revealed that more than $60 \%$ of young survivors experience reduced frequency of sexual intercourse since the time of diagnosis (Geue et al., 2015). This is often influenced by the feeling of being less attractive and by higher concerns about physical appearance (Paterson et al., 2016). Sexual difficulties are not short-term for this group. Sexual problems begin immediately after surgery, and although for some women they gradually decrease over time, they are still problematic one year and five year post-treatment (Baucom et al., 2005; Bloom et al., 2012; Burwell, Case \& Kaelin, 2006; Fobair et al., 2006).

Compared to their healthy peers, younger breast cancer survivors tend to report reduced sexual interest, decreased arousal, lower frequency of orgasm and less ability to relax (Herbenick, Reece, Hollub, Satinsky \& Dodge, 2008). Furthermore, they experience worse sexual relationships across the cancer continuum (Champion et al., 2014; Morrow et al, 2014; Paterson et al., 2016). However, greater involvement from the partner has been associated with better sexual adjustment (Wimberly, Carver, Laurenceau, Harris \& Antoni, 2005). As highlighted by Bakewell and Volker (2005), a woman's perceived degree of social support and quality of intimate relations are the strongest predictors of long-term sexual adjustment for this group of patients.

\section{Impact of Breast Cancer on the Couple Relationship of Younger Patients}

Despite the information reviewed in the previous paragraphs and the literature that supports the positive effect of close relationships in the adaptation to illness (Badr \& Krebs, 2013; Martire et al., 2010, Regan et al., 2012; Revenson \& DeLongis, 2011; Traa 
et al., 2015b), only a limited number of studies investigated the experience of younger patients and their partners while coping with breast cancer, with the majority of the contributions including only self-reported data from the patient (Borstelmann et al., 2015; Antoine et al, 2012; Burwell et al., 2008; Champion et al., 2014; Walsh et al., 2005).

Current data indicate that the presence of close relationships and social support has a protective role for the physical and psychological well-being of this group, with greater quality of life and lower levels of depression and anxiety for participants involved in satisfactory relationships (Borstelman et al., 2015; Christophe et al., 2015a; Christophe et al., 2015b; Hoyer et al., 2011; Gold et al., 2015; Vanlemmens et al., 2012a; Vanlemmens et al., 2012b; Wyatt, Beckrow, Gardiner, \& Pathak, 2008). However, the literature suggests that not all couples cope effectively with the stress of cancer. In a large prospective cohort study of women diagnosed with breast cancer at age 40 or younger, approximately $20 \%$ perceived the partner as unsupportive. For them, an increased likelihood to report anxiety symptoms existed (Borstelmann et al., 2015). Similarly, Avis, Crawford, and Manuel (2004) found that young women with higher levels of marital problems tend to report lower global, physical, emotional, and breast cancer-specific quality of life. This finding extends to survivorship, as younger survivors perceive less intimate or partner support than the older group, more social constraints and lower marital satisfaction (Champion et al., 2014; Stava, Lopez, \& Vassilopoulou-Sellin, 2006). For those experiencing difficulties in their relationship, younger survivors of breast cancer showed reduced perceived benefit from the cancer experience and higher negative impact on their well-being and quality of life (Champion et al., 2014). Finally, Walsh, Manuel, and Avis (2005) documented that although most women experienced higher 
closeness with their partners, 1 in 4 participants reported increased relational strain which ended in separation or end of the relationship $12 \%$ of the time.

Numerous authors affirm that male partners of younger women can be overwhelmed with multiple and competing caregiving demands, higher depressive symptoms, and worse quality of life (Antoine et al., 2012; Baucom et al., 2005;

Duggleby, Doell, Cooper, Thomas, \& Ghosh, 2014; Fergus et al., 2015; Hasson-Ohayon, Goldzweig, Dorfman, \& Uziely, 2014). Only three studies were identified to include both younger breast cancer patients and their partners (Antoine et al., 2012; Vanlemmens et al., 2012a; Vanlemmens et al., 2012b). In a qualitative analysis, Antoine et al. (2012) found that partners tended to be very close and supportive at the beginning of the cancer experience, providing high levels of intimacy and mutual support, while over time they wished for the couple to resume a sense of normalcy. In two other studies investigators tried to measure the impact of breast cancer on the quality of life of younger women and their partners' (Vanlemmns et al., 2012a; Vanlemmens et al., 2012b). Sixty-nine couples were interviewed. Results stressed the impact of the disease on psychological, physical, relational, social, sexual, domestic, professional and economic dimensions. These were mostly negative and still impacting the dyad even after treatment. Couple relationship and psychological well-being were the two dimensions addressed most of the time, with differences reported between the two genders. The analysis of the principal factors in the two resulting questionnaires confirmed these initial findings: in the patients' questionnaires the factor "feeling of couple cohesion" explained $19.8 \%$ of the total variance, while in the partners' questionnaires greater relevance was given to the management of the children and of the family's everyday life, the negative affectivity 
originated by the disease, and the following apprehension about the future (Christophe et al., 2015a; Christophe et al., 2015b). It is then possible to affirm that, for younger couples coping with breast cancer, the experiences tend to diverge among the two partners, with relationships taking a central role for patients while caregiving concerns and worries about the future become more relevant for the younger partners.

These indications are also confirmed by the literature examining coping and marital functioning among younger dyads and previous findings in the dyadic coping literature. Younger couples' relationships present higher vulnerability to distress, poorer quality of life, negative adaptation and coping abilities (Avis et al., 2005; Baucom et al., 2005; Berg \& Upchurch, 2007; Fobair et al., 2006; Iafrate, Bertoni, Donato \& Finkenauer, 2012; Revenson, 2003; Revenson \& Pranikoff, 2005; Thornton \& Perez, 2006). However, they have been largely overlooked by the empirical literature and limited knowledge is available about their psychsocial adaptation to cancer. While the development and evaluation of psychosocial interventions for this group have been recommended since the last decade (Baucom et al., 2005; Harden, Northouse, Cimprich, Pohl, Liang \& Kershaw, 2009; Regan et al., 2012), only two psycho-educational programs have been established to target their distinctive needs and the most recent one is currently under evaluation (Bloom et al., 1999; Fergus et al., 2014; Fergus et al., 2015).

Hence, the present work addresses a significant gap in the literature about couples coping with cancer, which is the attention to the developmental trajectory of dyadic coping over the life-span of the dyad and its impact on health and well-being (Aldwin, Skinner, Taylor, \& Zimmer-Gembeck, 2011; Berg \& Upchurch, 2007; Fingerman, Berg, Smith, \& Antonucci, 2001; Revenson \& Lepore 2012). This study will contribute to 
understand how younger patients and partners cope with a diagnosis of breast cancer, if they are more negatively affected than older couples, and how the individual's quality of life is influenced by relational characteristics and dyadic coping styles at different stages of life.

\section{Cancer as a Relational Illness: The Interdependence of Patients and Partners}

The importance of assuming a relational perspective in psychosocial oncology research is supported by the fact that one of the unique aspects of the cancer experience is the interdependence of partners' responses and coping strategies. As patients' adjustment is greatly influenced by interpersonal closeness and the quality of significant relationships, partners have an essential role in providing physical, emotional, spiritual or practical support (Lewis, 2010; Northouse \& McCorkle, 2010; Schover et al., 1997; Scott \& Kayser, 2009b; Varner, 2015). Similarly, patients’ reaction to cancer influences partners' physical and psychological well-being (Götze, Brähler, Gansera, Polze \& Köhler, 2014; Kim, Carver, Shaffer, Gansler, \& Cannady, 2015; Varner, 2015).

The literature about caregiving and interpersonal relationships in the context of cancer indicates that caregivers experience numerous types of burden including physical, psychosocial and financial difficulties (Deshields et al., 2012; Fletcher et al., 2008;

Grunfeld et al., 2004; Osse et al., 2006; Song et al., 2011; Stenberg, Ruland, \& Miaskowski, 2010; Williams \& McCorckle, 2011). Higher risks for stress and burnout have been reported in numerous contributions, with spouses' emotional distress higher or as high as the levels reported by the patients (Fitzell \& Pakenham, 2010; Hagedoorn et al., 2008; Manne et al, 2008; Thornton, Perez \& Meyerowitz, 2004; Wagner, Tanmoy, 
Bigatti \& Storniolo, 2011). Moreover, caregivers experience high levels of anxiety and depression, impaired sleep and other health-related issues, as evidenced by an increased use of primary care physicians especially in the context of breast cancer (Götze et al., 2014; Heins, Schellevis, Rijken, van der Hoek, \& Korevaar, 2013). The responsibilities associated with caregiving affect also health-promoting behaviors, with less than optimal levels of physical activity attributable to the role and its demands (Beesley et al., 2011; Mazanec et al., 2011).

The bond between patients and their significant ones is particularly evident as caregivers are called to adjust to the impact of the diagnosis on the individual's wellbeing, participate in the decision-making about cancer treatment, and cope with changes in occupation and family organization (Fitch \& Allard, 2007; Houldin, 2007; Lewis, 2010; Mellon, Northouse, \& Weiss, 2006; Northouse et al., 2000; Varner, 2015). While managing these issues, partners have to cope with the potential loss of their loved one and face the resulting sense of helplessness (Lewis et al., 2008). Although the emotional and social needs of informal cancer caregivers have been overlooked in the past (Institute of Medicine, 2008), the presence of supportive partners is crucial for the psychosocial wellbeing of patients and dyads over time (Carver et al., 2006; Kim et al., 2015; Neuling \& Winefield, 1988; Northouse, 1988; Roberts, Lepore \& Helgeson, 2006), and it will be examined in the next paragraphs. 


\section{The Impact of Cancer on the Couple Relationship \\ Quality of Life and Psychological Well-being}

The quality of life of both partners is negatively impacted by the illness, with patients and partners' scores being significantly lower than the healthy population (Bergelt, Koch \& Petersen, 2008; Dumont et al., 2006; Hinnen, Hagedoorn, Ranchor, \& Sanderman, 2008; Lewis, 2010; McClure, Nezu, Nezu, O’Hea, \& McMahon, 2012; Northouse \& McCorkle, 2010; Nakaya et al., 2010; Zenger et al., 2010). Impairments in emotional, physical and social areas have been documented for patients, while partners are at higher risk for worse health-related quality of life, anxiety and illness intrusiveness over time (Bergelt et al., 2008; Gotze et al., 2014; Kuenzler, Hodgkinson, Zindel, Bargetzi \& Znoj, 2011; Leung, Pachana \& McLaughlin, 2014).

A pattern of interaction, although moderate, has been identified between the distress of the members of the dyad (Baucom et al., 2012; Gotze et al., 2014; Kim et al., 2008; Mertz et al., 2011; Wahwa et al., 2013), suggesting that the stress associated with cancer is interpersonal in nature. These results have been confirmed also in longitudinal studies, where an over-time association between partners' distress and similar trajectories of functioning within the couple have emerged (Kim, van Ryn, Jenses, Griffin, Potosky, \& Rowland, 2015; Kraemer, Stanton, Meyerowitz, Rowland \& Ganz, 2011; Traa et al., 2014; Traa et al., 2015a; Segrin et al., 2005; Segrin \& Badger, 2014). Distress scores of patients and partners are not only associated, but some studies have identified that distress is the strongest predictor of quality of life (Kim et al., 2008). Hence, it is possible to affirm that the couple reacts to cancer as an emotional system, with a reciprocal influence on each other's well-being and interdependent emotional responses (Hagedoorn 
et al., 2008; Northouse \& McCorkle, 2010; Varner, 2015). Psychological distress is an important element of risk for the couple coping with cancer, because of its association with negative communication, reduced intimacy and worse emotional well-being (Manne, Badr, \& Kashy, 2012). It has also been established in bio-behavioral health research that difficulties in marital or intimate functioning have consequences on physiological indicators of distress, like endocrine and cellular immunity (Dorros, Card, Segrin, \& Badger, 2010; Heffner, Kiecolt-Glaser, Loving, Glaser, \& Mlarkey, 2004; Kiecolt-Glaser, Bane, Glaser \& Malarkey, 2003; Kiecolt-Glaser \& Newton, 2001). The interdependence of the partners' quality of life has been supported also when depression, anxiety, physical and mental health have been investigated. Graca Pereira, Figuereido, and Fincham (2012) found that higher scores on depressive symptoms were registered among partners of depressed cancer patients, while in a more recent study by Drabe,Wittman, Zwahlen, Buchi, and Jenewein approximately $40 \%$ of variance in female patients' depression was explained by partners' stress and coping resources (Drabe, Wittman, Zwahlen, Buchi \& Jenewein, 2013). Low level of functioning in general life domain had a spillover effect on the marital and sexual quality of the relationship (Traa et al., 2015a). Better quality of life of caregivers was associated with patients' higher mental health and better physical well-being (Wadhwa, Burman, Swami, Rodin, Lo \& Zimmerman, 2011). This association is confirmed by a more recent contribution (Litzelman \& Yabroff, 2015) where cancer survivors whose spouses reported depressed mood at time of diagnosis were more likely to report depression again eight years later. In the same study better mental and physical health-related quality of life of partners at baseline were associated to a $30 \%$ reduction in survivors' depressed mood risk, indicating 
that depression and poor health quality of life in partners may increase risk of depression for cancer patients, especially for women (Litzelman \& Yabroff, 2015).

In terms of predictors or correlates of distress, the role of cancer-related variables, demographic and psychological factors has been well-established by the literature. More recently, the focus of attention has shifted also toward relational factors (McLean \& Jones, 2007). Poor conflict resolution skills, pre-illness marital dissatisfaction, lower quality of family functioning, higher conflict rate, low social support, and different perceptions and expectations about the disease are associated with worse psychological well-being for both partners, and higher physical symptoms in the patient (Giannousi, Karademas, \& Dimitraki, 2016; Leung, Pachana \& McLaughlin, 2014; Mertz et al., 2011). Relationship quality was also predictive of better quality of life in partners of women with breast cancer, while higher mental functioning of the patient significantly contributed to greater physical and mental well-being in the spouse (Bergelt, Koch \& Petersen, 2008). Among partner's characteristics associated with patients' worse quality of life, there are critical and avoidant behaviors, reduced communication, and emotional withdrawal (Walsh et al., 2005). On the contrary, partner's distress has been associated to older age, lower level of education, increased concerns for the patient's well-being, caregiving burden and reduced marital satisfaction (Braun, Mikulincer, Rydall, Walsh, \& Rodin, 2007; Braun, Hales, Gilad, Mikulicer, Rydall, \& Rodin , 2012; Lewis et al., 2008; Lewis, 2010).

\section{Communication}

The ability of the couple to communicate about the illness and its consequences has been investigated widely in the literature because of its implications for adjustment and 
positive meaning-making. It has been proved that cancer significantly changes the communication patterns of the dyad, with modifications in communication abilities that have been associated with increased marital dissolution, reduced satisfaction and lack of social support (Badr, Acitelli, \& Taylor, 2008: Caughlin, 2002; Reynolds \& Perrin, 2004).

Despite these aspects of risk, a large body of evidence has demonstrated that open communication constitutes a resource for the couple (Lewis, 2010; Northouse et al., 2007; Porter, Keefe, Hurwitz \& Faber, 2005). Mutual constructive communication, selfdisclosure and more frequent relationship-talk have been consistently associated with higher levels of social support, quality of life, better psychological adjustment and relationship functioning for both cancer patients and partners (Badr et al., 2008; Langer, Brown, \& Syrjala, 2009, Manne et al., 2007; Manne et al., 2012; Porter et al., 2005; Song et al., 2012). Sharing feelings and concerns has a protective effect on the psychological well-being of cancer patients even when they are experiencing multiple physical symptoms (Manne, Badr, Zaider, Nelson, \& Kissane, 2010; Manne et al., 2012; Manne et al, 2015; Robbins, Lopez, Weihs \& Mehl, 2014).

However, differences in communication outcomes have been registered between patients and partners. Over time greater levels of communication about the couple relationship were associated to less distress in the partner (Badr, Acitelli \& Taylor, 2008), while for breast cancer patients the ability to display positive support-receiving behaviors was negatively impacted by the emotional arousal of the partner (Fischer et al., 2015). The inability of the couple to express their cancer-related concerns and emotions leads to increased psychological distress, strained marital relationships and compromised 
psychological adjustment (Manne et al., 2010; Julkunen, Gustavsson-Lilius \& Hietanen, 2009; Porter et al., 2005; Song et al. 2012). The underlying relationship between communication, individual and relational outcomes has been investigated in crosssectional and longitudinal studies, which highlighted that more positive spousal communication contributes to higher satisfaction through the reciprocal reinforcement of the two partners' intimacy (Manne et al., 2010; Manne et al., 2012; Manne et al., 2015).

However, disclosure of thoughts and feelings can be harmful under certain circumstances. For example dissimilar communication patterns among the two partners, like demand-withdraw communication, mutual avoidance and the provision of solutions from the healthy partner, have been associated with higher distress, depression, anxiety, and lower relationship satisfaction (Manne et al., 2010; Pasipanodya et al., 2012; Yu \& Sherman, 2015). For example some investigators found that emotional disclosure among partners did not attenuate their depressive symptoms. On the contrary, higher depression scores were identied at follow-up when members of the dyad divege in their amount of disclosure (Regan, Lambert, Kelly, Falconier, Kissane, \& Levesque, 2015a). The negative effect on the couple relationship was identified even when protective buffering was implemented with the goal to prevent negative emotions (Langer et al., 2009; Manne et al., 2007).

\section{Sexuality and Intimacy}

Although this area of the couple relationship has been previously neglected by the literature, a growing body of evidence has established that cancer dramatically impacts sexual functioning of patients and partners across a variety of cancer types ${ }^{2}$. Data show

\footnotetext{
${ }^{2}$ For a review please see the following publications: Badr \& Taylor, 2009; Charif et al., 2015; Den Oudsten et al., 2012; Den Oudsten, Van Heck, Van der Steeg, Roukema \& Vries, 2010; Flynn et al., 2011; Gilbert,
} 
that approximately half of all patients will encounter a sexual problem during their experience with cancer (Huyghe, Sui, Odensky, \& Schover, 2009), and that among breast cancer patients this percentage approaches $85 \%$ (Ussher, Perz, Gilbert, Hawkins, \& Wong, 2012).

Changes in sexual frequency and sexual satisfaction in women with breast cancer have been associated with physical consequences of cancer treatment, psychological factors, body image concerns and relationship characteristics (Carr, 2013; Manganiello, Hoga, Reberte, Miranda, \& Rocha, 2010; Moreira \& Canavarro, 2013; Ussher et al., 2015). Sex specific difficulties are often associated with isolation, anxiety, depression and sense of inadequacy (Anllo, 20007; Germino, Fife \& Funk, 1995; Hawkins et al., 2009; Rolland, 1994). Furthermore, for women who already present personality traits characterized by high levels of anxiety, this personality characteristic represents an independent predictor of worse quality of sexual life, sexual functioning and sexual enjoyment while coping with cancer (Den Oudtsen et al., 2010).

The impact of the disease on the partner is also considerable (DeGroot et al., 2005; Perez, Skinner \& Meyerwitiz, 2002; Reichers, 2004): fear of initiating sex and difficulties re-building a sense of normality in the sexual relationship with the patient have been reported (Maughan, Heyman, \& Matthews, 2003; Sanders et al., 2006). The dynamics of the caregiving relationship often interfere with the couple's intimate relationship: as the male partner is often providing physical care for the female patient, it becomes challenging to consider the woman as a sexual partner (Carr, 2013; D'Ardenne, 2004;

Ussher \& Perz, 2010; Hawkins, Ussher, Gilbert, Perz, Sandoval \& Dundquist, 2009; Kim et al., 2015; Lindau et al., 2011; Loaring, Larkin, Shaw \& Flowers, 2015; Milbury \& Badr, 2012; O’Brien, Roe, Low, Deyn \& Rogers, 2012; O’Shaughnessy, Ireland, Pelentsov, Thomas \& Esterman, 2013; Panjari et al., 2012 ; Ussher, Perz, Gilbert \& Australian Cancer and Sexuality Study Team, 2015; Wittman et al., 2015. 
Hawkins et al., 2009). The presence of sexual dysfunctions has been associated with poorer psychological adjustment (Levin et al., 2010), worse quality of life and increased risks of depression in partners (Nakaya et al., 2010). In a more recent study, Moreira and Canavarro (2013) confirmed the presence of impaired psychological adjustment of partners of women with breast cancer. However, the authors also highlighted that higher levels of intimacy were predictive of reduced depression and greater quality of life for both members of the couple.

Impaired sexual functioning has negative effects on the couple relationship (Carr, 2013; Badr \& Taylor, 2009; Galbraith et al., 2008; Ussher et al., 2015). Most couples experience cessation or decreased frequency of sex and intimacy, with only a small group of partners able to renegotiate new forms of sexual activity (Hawkins et al., 2009). The inability of the couple to recover significantly affects the display of other forms of intimate physical contact because these behaviors are perceived as leading to sex (Hughes, 2000; Kuyper \& Wester, 1998). On the contrary, couples able to negotiate new forms of intimacy reported increased communication, satisfaction and closeness in their relationship (Gilbert et al., 2010; Gilbert et al., 2011; Loaring, Larkin, Shaw, \& Flowers, 2015).

\section{Adjustment and Benefit Finding}

As reviewed in the previous paragraphs, most studies have focused on the negative effect the cancer diagnosis has on the individual and the couple relationship. However, couples also may find benefit from the cancer experience, a phenomenon called "posttraumatic growth". Defined by Tedeschi et al. (1996) as the "positive psychological change experienced as a result of the struggle with highly challenging life circumstances" 
(Tedeschi \& Calhoum, 2004, p. 1), post traumatic growth is associated with increased satisfaction with interpersonal relationships, closeness and empathy (Cordova, Cunningham, Carlson, \& Andrykowski, 2001; Weiss, 2002; 2004).

When measured in dyads, growth scores in cross sectional and longitudinal studies are not only associated between the partners (Manne et al., 2004; Thornton \& Perez, 2006; Weiss, 2004; Zwahlen et al., 2010), but growth reported by the female cancer patient is among the predictors of the husband's growth (Weiss, 2004). It is interesting to report that mixed results have been presented about levels of personal growth. While Kunzler, Nussbeck, Moser, Bodenmann, \& Kayser (2014) indicate higher scores for women (either as patients and partners), in a cross-sectional study by Cormio et al. (2014), caregivers showed significantly higher levels of improvements. This result was explained by the researchers with the positive effect of providing care to the patient. This is consistent with other studies where investigators have found that assuming the role of caregiver produced numerous benefits like increased positive self-view, higher empathy and appreciation, changes in priorities and values (Cassidy, McLaughlin \& Giles, 2014; Kim, Schultz \& Carver, 2007; Lin, Fee, \& Wu, 2012). Researchers were also interested in examining if differences in growth and meaning-making were influenced by gender, role or dyad factors. Findings indicate that intra-couple characteristics influence the positive identification of benefits and that partners may experience comparable growth (Zwahlen et al., 2010), with variability between couples being larger than the variability associated to gender or role.

It is then possible to state that couples share not only the burden of the illness, but also the possibility to grow as individuals and to enhance their relationship (Fergus \& 
Gray, 2009; Traa et al., 2015b; Regan et al., 2015a). A significant association between coping styles, partners' well-being and marital satisfaction has been extensively confirmed in the literature across populations and disciplines (Traa et al., 2015b; Regan et al., 2015a; Iafrate \& Donato, 2012). Positive adjustment to a variety of stressors is associated with active engagement and problem-focused coping strategies, while protective buffering, denial and wishful thinking lead to adverse psychosocial outcomes, like reduced relationship satisfaction and worse mental well-being (Berg et al., 2008; Kraemer et al., 2011; Langer et al., 2009; Merz et al., 2011; Romero et al., 2008).

The process of adaptation to the illness is complex and affects both members of the couple (Fekete et al., 2007; Kayser \& Sormanti, 2002; Kayser, Sormanti, \& Strainchamps, 1999; Langer et al., 2009; Regan et al., 2015a; Skerret, 1998; Traa et al., 2015b). From several contributions, it clearly emerges that the ability of the couple to share this experience leads to enhanced couple functioning (Kayser et al., 2007; Skerret, 1998). It is now well-established in the literature that cancer patients report better psychological adjustment to illness, physical well-being and emotional adjustment if they experience their partners as being emotionally supportive. The spouse or the significant other is often the primary supportive figure during the cancer experience and, despite the availability of other sources of support, none of these relationships can compensate for his/her absence (Cutrona, 1996a; 1996b; Northouse \& McCorkle, 2010; Varner, 2015). Coping strategies used by each partner significantly influence the overall adaptation of the dyad over time. Better adjustment is related to the display of problem-focused coping strategies, collaborative coping and active engagement (Llewellyn, Horney, Gurk, Weinman, Herold, Altman \& Smith, 2013; Regan et al., 2015a; Romero et al., 2008; Traa 
et al., 2015b). On the contrary, higher level of distress, illness intrusiveness and lower psychosocial adjustment are registered when patients experienced partners as overprotective or dismissive of their needs (Donato, 2012; Feldman \& Broussard, 2005; 2006; Kuijer, 2000). It is therefore possible to conclude that strong evidence from the literature supports that assuming a relational perspective is necessary when exploring the impact of cancer on patients and partners.

\section{Summary}

The present chapter offered a review of the literature related to the impact of breast cancer on the quality of life of younger women and partners. Additionally, the chapter provides an empirical justification for assuming a relational perspective when working

with couples coping with cancer by examining the literature about quality of life, distress, communication, sexuality and benefit finding. 


\section{CHAPTER II: LITERATURE REVIEW}

This chapter explores the foundational literature of dyadic coping and introduces the theoretical framework of the proposed research study. In the first part, current models of dyadic coping are reviewed and the conceptual framework that forms the basis for the proposed research is presented. In the second section of the chapter the relevance, strengths, and limitations of Systems Theory, Stress and Coping Theory, RelationalCultural Theory and Life Course Developmental Framework to study dyadic coping and the experience of younger couples are discussed.

\section{Dyadic Coping and its Relevance for the Marital Relationship in a Time of Crisis}

In the last twenty years a new attention towards interpersonal aspects of coping has emerged (Donato, 2012; Iafrate \& Donato, 2012; Kayser \& Scott, 2008; Saita, 2009; Regan et al., 2015a; Revenson, Kayser \& Bodenmann, 2005; Traa et al., 2015b). This new interest has created the condition for the theoretical frameworks of close relationships and stress and coping to converge with the goal to examine how coping develops within the context of significant relationships (Iafrate \& Donato, 2012; Revenson et al., 2005). As a consequence, couples' coping has started to be conceptualized no longer at the individual level -referring to the separate perspectives of 
the two partners- but as a dyadic process involving the partners' mutual influence (Bodenmann, 1997).

The literature supports the idea that dyadic coping should be conceptualized as a process shaped by the context of close relationships (Bodenmann, 2005; Peterson \& Bush, 2013; Revenson et al., 2005). It is described as "the interplay between the stress signals of one partner and the coping reactions of the other, a genuine act of shared coping” (Revenson et al., 2005; p. 4). Through a series of interactions, dyadic coping contributes to a sense of we-ness and promotes the conjoint creation of strategies to respond to the stressful event (Bodenmann, 1997; Kayser et al., 2007; Revenson, 1994; Scott, Halford, \& Ward, 2004). As a circular and bi-directional process, it is based on the assumption that both partners are equally involved in the coping process: they are providing and receiving support from each other while engaging in joint problem-solving activities and shared emotion regulation (Bodenmann, 1995a; Revenson \& DeLongis, 2011; Bodenmann, 2005; Revenson \& Lepore, 2012).

The association between dyadic coping and marital functioning, better psychological and physical well-being, and lower stress has been established across different populations and couples coping with a variety of stressors (chronic illnesses, depression, anxiety, anger and verbal aggression) (Badr, Carmack, Kashy, Cristofanilli, \& Revenson, 2010; Bodenmann, Atkins, Schar, \& Poffet, 2010; Bodenmann, Charvoz, Widmer, \& Bradbury, 2004; Hinnen et al., 2008; Randall \& Bodenmann, 2009; Sullivan, Pasch, Johnson, \& Bradbury, 2010; Vilchinsky \& al., 2010). Ambivalent or hostile coping styles are more frequent among distressed couples or in the context of mental disorders and chronic illness (Bodenmann, 1995; 2000; Bodenmann et al., 2004). On the 
contrary, positive dyadic coping can reduce the negative impact of stress on a relationship (Martin, Peter-Wight, Braun, Hornung, \& Scholz, 2009). The role of dyadic coping as protective factor for couples' functioning over time has been confirmed by longitudinal studies, with higher relational satisfaction and reduced odds of being divorced for couples reporting common positive coping (Bodenmann \& Cina; 2005; 2006; Bodenmann, Pihet, \& Kayser, 2006). More recently, the most comprehensive meta-analysis to date provided stronger evidence for the fact that dyadic coping significantly predicts relationship satisfaction and that aggregated positive forms of dyadic coping were stronger predictors of this outcome than negative forms (Falconier, Jackson, Hilpert, \& Bodenmann, 2015).

As anticipated in the previous chapter, when considering the stress of a cancer diagnosis the ability of the couple to face the illness as a unit contributes to higher relationship quality and cohesion (Badr et al., 2010; Fergus \& Gray, 2009; Picard, Dumont, Gagnon, \& Lessard, 2005; Traa et al., 2015b). Similarly, relationship maintenance behaviors, social support exchanges, mutual constructive communication and joint dyadic coping have been associated to better relationship functioning and quality of life (Badr \& Taylor, 2009; Hagedoorn et al., 2011; Langer et al., 2009; Lavery \& Clarke, 2009; Manne et al., 2015; Norton \& Manne, 2007; Pasipanodya et al., 2012).

However, it would be inaccurate to address dyadic coping as a unitary phenomenon, since over the years several models have been developed in the literature (Bodenmann, Meuwly, \& Kayser, 2011; Donato 2012; Folkman, 2011; Kayser, Acquati, Saita \& Costa, 2011; Hubbard et al., 2013; Iafrate \& Donato, 2012; Levesque et al, 2014b; Regan et al., 2015a; Revenson \& Lepore, 2012; Traa et al., 2015b). Although they all share the same view of dyadic coping as a process through which partners cope with the stress they 
encounter in their life as a couple, each offers unique insight on what dyadic coping is and how it is associated with relational and individual well-being (Donato 2012; Iafrate \& Donato, 2012; Kayser et al., 2011; Scott \& Kayser, 2009b; Regan et al., 2015a; Traa et al., 2015b). The most recent literature has tried to explore and compare these theoretical frameworks and to clarify the relationship between dyadic coping and couple relationship in the context of cancer (Hubbard et al., 2013; Regan et al., 2015a; Traa et al., 2015; Zimmerman, 2015). However, the contemporary lack of consensus in the conceptualization and assessment of dyadic coping confirms the complexity of the study of human relations during a time of crisis, such as the experience of a serious and debilitating illness (Table 1). Despite this complexity, the current debate about what dyadic coping is, its relationship with relational outcomes, daily stressors and social support as well as the theories that have shaped its conceptualization is extremely needed and promising for psychosocial oncology. Furthermore, an ongoing critical reflection on dyadic coping is crucial to completely understand the relationship between stress and health (Revenson, 2003; Revenson \& DeLongis, 2011; Revenson \& Lepore, 2012). 
Table 1. Overview of Dyadic Coping Models

\begin{tabular}{|c|c|c|c|}
\hline Models & Authors & Definition of Dyadic Coping & Theoretical Framework \\
\hline $\begin{array}{l}\text { Relationship-Focused } \\
\text { Coping }\end{array}$ & Coyne \& DeLongis & $\begin{array}{l}\text { Relational-focused or relational coping refers to cognitive and } \\
\text { behavioral efforts to maintain and protect social relationships. } \\
\text { Coyne: active engagement and protective buffering. } \\
\text { DeLongis: empathic and interpersonal-withdrawal coping }\end{array}$ & $\begin{array}{l}\text { Stress \& Coping Theory } \\
\text { Systems Theories } \\
\text { Social Learning Theory }\end{array}$ \\
\hline Congruence Model & $\begin{array}{l}\text { Barbarin, Hughes, \& } \\
\text { Chesler } \\
\text { Revenson }\end{array}$ & $\begin{array}{l}\text { Focus is on congruency of problem-focused and emotion-focused } \\
\text { individual coping strategies among partners. Importance of } \\
\text { ecological/contextual perspective. Congruence can involve similar or } \\
\text { complementary coping styles. }\end{array}$ & $\begin{array}{l}\text { Person-Environment Fit } \\
\text { Theory } \\
\text { Family Systems Theories } \\
\text { Earlier work on } \\
\text { Relational Coping }\end{array}$ \\
\hline $\begin{array}{l}\text { Systemic-Transactional } \\
\text { Model }\end{array}$ & Bodenmann & $\begin{array}{l}\text { Dyadic coping is viewed as a circular process activated in response of a } \\
\text { dyadic stress. Both positive (supportive, common, and delegated) and } \\
\text { negative (hostile, ambivalent, superficial) forms of dyadic coping exist. }\end{array}$ & $\begin{array}{l}\text { Stress \& Coping Theory } \\
\text { Systems Theory }\end{array}$ \\
\hline $\begin{array}{l}\text { Relational-Cultural } \\
\text { Model }\end{array}$ & $\begin{array}{l}\text { Kayser, Watson \& } \\
\text { Andrade }\end{array}$ & $\begin{array}{l}\text { Dyadic coping is a process that occurs multiple times during the cancer } \\
\text { experience. Relational characteristics (relationship awareness, } \\
\text { authenticity, and mutuality) determine the pattern of coping the couple } \\
\text { develops. Two patterns of relational coping: mutual responsiveness or } \\
\text { disengaged avoidance. }\end{array}$ & $\begin{array}{l}\text { Relational-Cultural } \\
\text { Theory } \\
\text { Systems Theory } \\
\text { Systemic-Transactional } \\
\text { Model of Dyadic Coping }\end{array}$ \\
\hline $\begin{array}{l}\text { Developmental- } \\
\text { Contextual Model }\end{array}$ & Berg \& Upchurch & $\begin{array}{l}\text { Dyadic coping changes through the life-span as a consequence of } \\
\text { developmental, temporal and contextual factors (culture and gender; } \\
\text { quality of the marital relationship and the demands of the chronic } \\
\text { illness). Collaborative coping, uninvolved strategies, supportive } \\
\text { strategies and control strategies. Functions of collaborative coping are } \\
\text { resource and relationship enhancement }\end{array}$ & $\begin{array}{l}\text { Social Development } \\
\text { Theory }\end{array}$ \\
\hline
\end{tabular}




\section{Relationship-focused coping}

The first step in the development of a dyadic conceptualization of coping is due to the work of James Coyne and Anita DeLongis (1986). While emotion-focused and problem-focused coping were initially introduced by Lazarus and Folkman in 1984, Coyne and DeLongis identified a third coping function: relational-focused or relational coping. With this term the authors emphasize the cognitive and behavioral efforts to maintain and protect social relationships while coping with stressful events (O’Brien \& DeLongis, 1997; Revenson \& DeLongis, 2011).

Two major frameworks have inspired the work of Coyne, Stress and Coping Theory (Lazarus \& Folkman, 1984) and Bandura’s Social Learning Theory (Bandura, 1986). While the Transactional Model of Stress and Coping has been the theory of reference for the study of coping strategies for more than thirty years, Coyne recognized that this approach was not able to capture processes that occur in couples coping with health problems (Coyne \& Friske, 1992). As a consequence, from his perspective Social Learning Theory appeared to be the approach better able to highlight the centrality of people's beliefs about their capacity to execute behaviors (self-efficacy) and their perseverance in the face of significant challenges (Bandura, 1986). Coyne and colleagues identified two broad categories of relationship-focused coping: active engagement and protective buffering (Coyne, Ellard \& Smith; 1990; Coyne \& Friske, 1992; Coyne \& Smith, 1991; Coyne, Wortman, \& Lehman, 1988). Active engagement refers to the active involvement of one partner in discussion, constructive problem-solving and attention to feelings (Coyne \& Smith, 1994). Protective buffering, on the contrary, describes the behavior of a partner that hides concerns, denies worries and tries to avoid disagreement 
with the other (Coyne \& Smith, 1991). Evidence from research studies applying this framework to couples coping with diverse stressors indicate that active engagement is positively associated to reduced psychological distress, higher self-efficacy, and higher relationship functioning (Hagedoorn et al., 2011; Kuijer et al., 2000). On the contrary protective buffering has been linked to negative relational and psychosocial outcomes (Coyne \& Smith; 1991; Hagedoorn et al., 2011; Kuijer et al., 2000; Manne et al., 2007).

Similarly, DeLongis and O'Brien (1990) identified dyadic coping as an interpersonal and regulatory process characterized by two opposite strategies, empathic and interpersonal-withdrawal coping (DeLongis \& O’Brien, 1990; Revenson \& DeLongis, 2011). Based on the assumption that maintaining relations is a fundamental human need that influences cognitions and emotions (Baumeister \& Leary, 1995; Bolger, DeLongis, Kessler, \& Schilling, 1989), empathic coping is described as the attempts of one partner to explore the emotional experience of the other and to behaviorally respond in a supportive and caring manner. Interpersonal-withdrawal coping, on the contrary, refers to behaviors and strategies enacted to prevent the partner from dealing with problems or experiencing the feelings of the other member of the dyad (DeLongis \& O'Brien, 1990; O’Brien \& DeLongis, 1996; O’Brien \& DeLongis, 1997; O’Brien, DeLongis, Pomaki, Puterman, \& Zwicker, 2009).

These authors have contributed to raise awareness of the fact that coping processes among partners involve the couple relationship and affect the quality of this relationship. Despite its popularity ${ }^{3}$, a little relationship-focused coping explains about the

\footnotetext{
${ }^{3}$ For a review if it possible to refer to these publications: Coyne, Wortman, \& Lehman, 1988; Coyne, Ellard \& Smith; 1990; Coyne \& Friske, 1992; Coyne \& Smith, 1991; Coyne \& Smith, 1994 DeLongis \& O’Brien, 1990; O’Brien \& DeLongis, 1996; O’Brien \& DeLongis, 1997; O’Brien, DeLongis, Pomaki, Puterman, \& Zwicker, 2009; Hagedoorn et al., 2011; Kuijer et al., 2000; Manne et al., 2007
} 
interdependence of the partners' coping processes. The model focuses on individual strategies, rather than on relationship characteristics and processes that promote partners' adjustment and well-being.

\section{Congruence Model}

The Congruence Model of dyadic coping is represented by the work of Barbarin, Hughes, and Chesler (1985) and Revenson (1994) who explored the congruency of problem-focused and emotion-focused individual coping strategies among partners (Peterson \& Bush, 2013). The theoretical reference for the work of these authors are Person-Environment Fit Theory (French, Rodgers, \& Cobb, 1974), Family Systems Theories (Patterson \& Garwick, 1994) and the earlier work on relationship-focused coping (Coyne \& Friske, 1992; Coyne \& Smith, 1991; DeLongis \& O’Brien, 1990). The model emphasizes that the couple constitutes a system in relation with outside forces and the importance of studying couples coping with an ecological and contextual perspective. Revenson (2003) highlights that her perspective describes psychosocial adaptation as a "function of the ecological niche the couple occupies" (Revenson, 2003; p. 535). Hence, the focus of attention should be broadened to include socio-cultural, interpersonal, situational and temporal contexts, with the ultimate goal to expose all the factors that influence couple's coping. By assuming an ecological approach, the model is then perceived as capable of addressing the reciprocal behavioral and psychological interdependence that characterizes the marital relationship (Revenson, 2005; 2003).

According to this view, congruency and discrepancy of individual coping strategies are connected to marital satisfaction and personal well-being (Revenson \& DeLongis, 2011). Revenson and colleagues affirm that couples coping has the objective to 
“maximize the congruence or fit between partners' coping styles in order to cope most effectively as a couple” (Revenson, Abraido-Lanza, Majerovitz, \& Jordan, 2005; p.141). Congruence can involve similar or complementary coping styles: both partners can use emotional or problem-solving coping, while partners may use emotional coping style in response to the problem-solving approach of the other spouse (Revenson 1994; 2003). When partners' strategies are similar, their coping efforts are coordinated and mutually reinforcing. However, this does not mean that complementary approaches are not congruent: the author highlights that complementarity can be effective as it provides a broader "coping repertoire" to the couple (Revenson, 2003; p. 540). Non-congruent strategies, on the contrary, occur when the partners' strategies are mutually opposite, aimed at "cancelling each other out" (Iafrate \& Donato, 2012; p. 8), and are associated with worse psychosocial outcomes.

Empirical evidence for the model has been provided by several studies conducted on healthy couples (Iafrate, Bertoni, Margola, Cigoli, \& Acitelli, 2011; Iafrate \& Donato, 2012) and couples coping with chronic illnesses ${ }^{4}$. The application of this conceptualization of dyadic coping to chronic illnesses like rheumatic disease (Revenson et al., 2005), multiple sclerosis (Pakenham, 1998; Upchurch at al., 2003), and COPD (Snippe, Maters, Wempe, Hagedoorn, \& Sanderman, 2012) has produced evidence for higher distress and lower levels of marital satisfaction for dyads presenting incongruent coping styles. More specifically, Revenson et al. (2005) identified four clusters of couples' coping (effortful partnership, problem solvers with emotion coping, minimalist

\footnotetext{
${ }^{4}$ For a review it is possible to refer to these publications: Ben-Zur et al., 2001; Kaptein \& al., 2007; Kienle, Luszczynska, Pfüller, \& Knoll, 2009; Revenson, 2003; Revenson et al., 2005; Sterba \& al., 2008;

Verhofstad, Ickes, \& Buysse, 2010; Watts et al., 2011; Yoshimoto et al., 2006
} 
copers and couples where the patient copes alone). Although individuals from the first cluster reported higher level of distress, they also rated higher in personal growth. Similarly, Snippe, Maters, Wempe, Hagedoorn, and Sanderman (2012) identified the emotional distress was independently associated with self-reported perceptions of COPD patients' use of protective buffering and discrepancies in the partners' overprotection.

Six studies of couples coping with cancer were inspired by the congruence model (Regan et al., 2015a). Overall, results indicate that congruent coping styles are associated with greater patient and partner adjustment to illness and marital satisfaction, while increased distress and reduced quality of life were reported otherwise (Banthia et al., 2003; Barnoy, Bar-Tal, \& Zisser, 2006; Ben-Zur, Gilbar \& Lev, 2004; Fagundes, Berg \& Wiebe, 2012; Norton \& Manne, 2007; Taylor et al., 2008). A more recent study examined couples' coping strategies as predictors of adjustment to breast cancer at 10 and 20 months post diagnosis. Within the 139 couples who participated in the study, women's use of approach-oriented coping strategies predicted improvement in their vitality and depressive symptoms, while men's avoidant coping predicted declining marital satisfaction for wives. As anticipated, the implementation of approach-oriented strategies among male partners was predictive of increased perception of cancer-related benefits in breast cancer patients. Among these couples, coping strategies also interacted to predict adjustment, with better adaptation originated by congruent coping as opposed to dissimilar styles (Kraemer et al., 2011).

Although researchers assuming this perspective believe that each partners' coping style is related to the other, the model still considers coping an individual phenomenon (Pakenham, 1998; Revenson, 1994; 2003; Revenson et al., 2005; Revenson \& DeLongis, 
2011). Hence, it is measured separately among the members of the dyad and the analyses tend to compare individual scores or to utilize the sum of the scores from the two partners (Pakenham, 1998; Revenson, 1994), without accounting for intrinsic interdependence of scores from individuals involved in a close relationship. Furthermore, despite the evidence presented in this paragraph and the model's heuristic value, inconclusive data have been collected about "whether congruent coping is better and what congruent coping is" (Revenson \& DeLongis, 2011; p.110; Regan et al., 2015a).

\section{Systemic-Transactional Model}

The Systemic-Transactional Model introduced by Guy Bodenmann in the late '90s distinguishes individual coping efforts, where the stress is managed independently, from dyadic coping processes in which both partners are involved (Bodenmann, 1995; 1997; 2000; 2005). In his approach, dyadic stress entails "any form of emotional or problemcentered stress directly concerning the couple as a unit” (Bodenmann, 1997, p. 138), which elicits their conjoint coping efforts (Bodenmann, 2005). Areas affected by dyadic forms of stress are partners' common concerns, their emotional intimacy and the continuity of their relationship (Bodenmann, 1994; 1997). Dyadic stressors can be classified along three dimensions: the way partners are affected by the event (directly or indirectly), the origin of stress (internal vs. external), and the time sequence (Bodenmann, 1995; 1997; 2005).

Dyadic coping is regarded as a circular process: through a stress communication exchange, the individual appraisal is communicated to the partner, whom then interprets and decodes the partner's stress signals and responds with a form of dyadic coping (Bodenmann, 1994; 1997). The function of dyadic coping is twofold: stress reduction and 
enhancement of the couple relationship (Bodenmann, 2005; Donato, 2012; Traa et al., 2015b). As the well-being of each member of the couple depends on the well-being of the other and the interaction with the larger social environment, dyadic coping becomes a strategy to manage the stress of both partners. Second, the ability of the partners to use positive forms of dyadic coping promotes a sense of we-ness, defined as "mutual trust, reliability, commitment and the perception that the relationship is a supportive resource" (Bodenmann, 2005, p. 41), which increases marital quality and stability (Bodenmann, 2005; Cutrona, 1996a; Cutrona, 1996b; Traa et al., 2015b, Regan et al., 2015a).

The author distinguishes between negative and positive forms of dyadic coping (Figure 1), resulting from event characteristics, individual and dyadic appraisal, and partners' competences (Bodenmann, 1995; 1997; 2005; Iafrate \& Donato, 2012). Common, supportive and delegated dyadic coping represent positive and adaptive dyadic coping styles, while negative dyadic coping is classified in hostile, ambivalent and superficial (Bodenmann, 1995; 1997; 2005). An overview is presented in Figure 1 and Table 2. 
Figure 1. Overview of Positive and Negative Forms of Dyadic Coping.

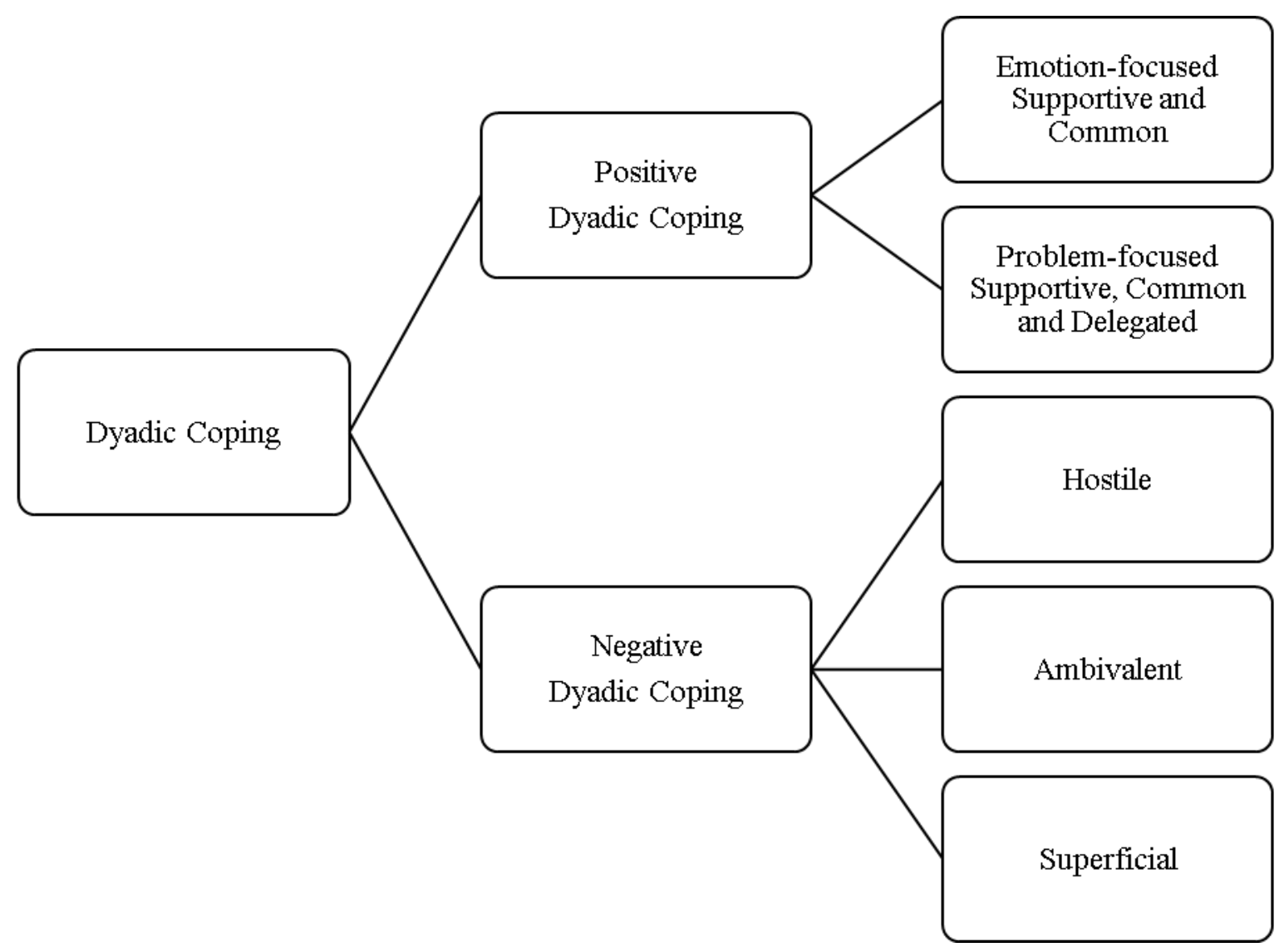

Table 2. Description of Positive and Negative Dyadic Coping Styles

\begin{tabular}{|c|c|}
\hline $\begin{array}{c}\text { Forms of } \\
\text { Dyadic Coping }\end{array}$ & $\begin{array}{c}\text { Description of } \\
\text { Dyadic Coping Styles }\end{array}$ \\
\hline \multirow{3}{*}{$\begin{array}{l}\text { Positive Forms of } \\
\text { Dyadic Coping }\end{array}$} & $\begin{array}{l}\text { Supportive Dyadic Coping: one partner assists the other with the } \\
\text { secondary goal to reduce his/her own distress. Examples of } \\
\text { supportive coping include practical activities, empathic } \\
\text { understanding and expressing solidarity. }\end{array}$ \\
\hline & $\begin{array}{l}\text { Common Dyadic Coping: both members of the couple } \\
\text { experience the stressful event and they participate in the coping } \\
\text { process in a symmetric or complementary way. They use } \\
\text { strategies like joint problem solving, information seeking, and } \\
\text { mutual commitment. }\end{array}$ \\
\hline & $\begin{array}{l}\text { Delegated Dyadic Coping: one partner is explicitly asked to give } \\
\text { support or to take over responsibilities in order to reduce the } \\
\text { stress of the other member of the couple. }\end{array}$ \\
\hline
\end{tabular}




\section{Negative Forms of Dyadic Coping}

Hostile Dyadic Coping: involves the provision of support which is accompanied by disinterest or minimizing the seriousness of the partner's stress.

Ambivalent Dyadic Coping: one partner supports the other but unwillingly or with the belief that his or her involvement is unnecessary.

Superficial Dyadic Coping refers to support which is insincere, distant or cold.

Four assumptions underlie the Systemic-Transactional Model. First, dyadic stress and coping are conceptualized from a systemic perspective, as one partner's stress appraisal influences and it is influenced by the other partner's and the relationship. Second, dyadic coping represents only one form of coping reported by the partners, as each of them still displays individual coping and supportive interactions with the larger social network. Third, from Bodenmann's perspective dyadic coping is a resource used by couples after individual coping styles have failed. Finally, the author differentiates dyadic coping from social support. The support provided by a partner is significantly different from and has different meanings from the support provided by other people. Dyadic coping is a conjoint commitment of both partners, while social support is only one form of dyadic coping among the multiple possible (Bodenmann, 2005).

This model has greatly contributed to the advancement of our understanding of dyadic coping and its role in marital functioning (Falconier et al., 2015; Revenson \& DeLongis, 2011; Traa et al., 2015b). Extensive literature through the years has supported the relationship between dyadic coping and greater marital satisfaction, better psychological and physical well-being, and reduced stress ${ }^{5}$-with dyadic coping

\footnotetext{
${ }^{5}$ For an overview it is possible to refer to the the following contributions Baas \& Schmitt, 2004; Badr et al., 2010; Bodenmann, 2000; 2005; Bodenmann, Pihet \& Kayser., 2006; Hochfilzer, 2010; Koch, Hauschild, \& Schmidt-Denter, 2001; Kunzler, Zindel, Bargetzi, \& Znoj, 2005; Schneewind \& Kruse, 2002; Schwarzer \&
} 
representing a stronger predictor of relationship functioning than individual coping strategies and communication- (Bodenmann, Bradbury \& Pihet, 2008; Papp \& Witt, 2010). These results have been confirmed also in longitudinal studies (Bodenmann \& Cina, 2006; Ruffieux, Nussbeck, \& Bodenmann, 2014).

Furthermore, dyadic coping attenuates the association between stress, anger and verbal aggression (Bodenmann, Meuwly, Bradbury, Gmelch \& Ledermann, 2010), and individuals tend to recover faster from an experimentally induced stress the more positive dyadic coping receive from the partner (Meuwly, Bodenmann, Germann, Bradbury, Ditzen \& Heinrichs, 2012). Support comes also from studies conducted on chronic illness (Acitelli \& Badr, 2005; Hagedoorn et al., 2008, Manne et al., 2007; Naumann, 2004; Rohrbaugh et al., 2002; Meier et al., 2012), immigration stress (Falconier, Nussbeck \& Bodenmann, 2013; Austin \& Falconier, 2013), sexual behavior (Bodenmann et al., 2010), and in cross-cultural samples (Bertoni et al., 2007; Ledermann et al., 2010) .

More recently, the mechanism through which dyadic coping protects the couple relationship has become the focus of attention. Among the alternatives presented in the literature, investigators have identified that we-ness partially mediates the relationship between coping and relationship satisfaction especially for women (Vedes, Nussbeck, Bodenmann, Lind, \& Randall, 2013), as well as the presence of a significant correlation between commitment and higher levels of common dyadic coping (Landis et al., 2014). Although the relationship between stress, dyadic coping and well-being has been

Bush, 2005; Wunderer \& Schneewind, 2008; Bodenmann. Meuwly \& Kayser, 2011; Meier, Bodenmann, Morgeli, \& Jenewein, 2011; Kramer, Ceschi, Van der Linden \& Bodenmann, 2005; Dehle, Larsen, \& Landers, 2001; and Walen \& Lachman, 2000. 
confirmed in studies inclusive of couples at different moments of their life-span ${ }^{6}$, questions remain about challenges experienced by different cohorts of couples (Vedes, Nussbeck \& Bodenmann, 2015; Landis, Peter-Wight, Martin \& Bodenmann, 2013).

Although this model has been extensively used by the close relationtioships literature, in both the reviews conducted by Traa et al. (2015b) and Regan et al. (2015a) only a few studies were identified to have applied the STM to cancer. Badr, Carmack, Kashy, Cristofanilli and Revenson (2010) conducted a longitudinal study of metastatic breast cancer patients and partners. Their findings indicated, as expected, that common positive dyadic coping was associated to more positive adjustment for both members of the dyad, while negative dyadic coping was associated to higher distress and reduced levels of adjustment. The association between negative dyadic coping and distress was higher for patients than for partners. Another study conducted on the same sample confirmed that dyadic adjustment was associated to better psychological well-being, as indicated by fewer depressive symptoms (Badr \& Shen, 2014). Similar findings were reported by Rottman et al. (2015). Their longitudinal examination of the relationship between dyadic coping, depression and relationship quality revealed in a large sample of dayds coping with breast cancer that negative dyadic coping affects patients and partners' individual and relational outcomes equally. Self-reported perceptions of engaging in common dyadic coping were related to higher relational quality and reduced number of depressive symptoms for both. However, differences occurred based on the type of coping style provided: while partners reported fewer depressive symptoms the more delegated coping they provided to the patient, the opposite trend was registered for

\footnotetext{
${ }^{6}$ Studies investigating dyadic coping have been conducted on late adolescent couples, middle-age and older-age couples.
} 
patients. Furthermore, if the patient provided partner with supportive coping, partners experienced higher levels of depression (Rottman et al., 2015).

Investigators have also applied the Systemic Transactional Model to prostate cancer. In a cross-sectional study of 42 pairs of patients and caregivers, Regan et al. (2014) found that the utilization of positive and negative dyadic coping was significantly associated to higher relationship satisfaction. On the contrary, anxiety and depression were associated to partners' self-reported perceptions of negative dyadic coping. In the same year, Lafaye, Petit, Richaud, Houede, Baguet, and Cousson-Gelie (2014) investigated the effects of coping strategies on emotional well-being and quality of life of prostate cancer patients and partners. Problem-focused coping strategies or social-support strategies were associated to reduced depression and anxiety for both partners, while the opposite was reported for emotion-focused coping (Lafaye et al., 2014). Finally, the dyadic adjustment of couples undergoing bone-marrow transplantation for cancer was examined by Fife, Weaver, Cook, and Stump (2013). Again, authors identified that dyadic coping styles aimed at promoting the relationship had the highest positive impact on adjustment over a 12-month period.

Since dyadic coping is considered a competence that can be increased, several programs have been developed to teach relational skills with the long-term goal to enhance couples' ability to learn and apply these behaviors (Schaer, Bodenmann, \& Klink, 2008; Bodenmann, Bradbury \& Pihet, 2008; Ledermann, Bodenmann, \& Cina, 2007; Bodenmann \& Randall, 2012). Significant improvements on communication and psychological well-being have been reported across diverse samples and cultures 
(Bodenmann \& Bertoni, 2004; Bodenmann, Bradbury \& Pihet, 2009; Bodenmann, Hilpert, Bradbury \& Nussbeck, 2014).

An aspect of limitation is represented by the fact that this approach still considers dyadic coping as a resource the couple utilizes when individual's coping mechanisms have failed. Also, it seems that the model focuses on the individual coping efforts, since it does not clarify whether and how the communication of the cognitive appraisal of the individual initiates a conjoint or shared reaction. Since this conceptualization has been developed within the close relationships literature, little attention has been given to forms of stress that change over time (i.e. stress that changes in intensity and requires the couple to develop new forms of management/adjustment), with major attention given to daily stressors. However, the contribution this perspective has brought to the study and measurement of dyadic coping is so relevant that the instrument developed from the Bodenmann's model will be utilized in the present study.

\section{Relational-Cultural Model}

A few years later, the Relational-Cultural Model of dyadic coping was introduced (Kayser et al., 2007). Inspired by the previous work of Kayser and colleagues in the context of breast cancer (Kayser \& Sormanti, 2002; Kayser, Sormanti, \& Strainchamps, 1999), this conceptualization focuses on the multiple stressors encountered at each stage of the cancer experience (diagnosis, treatment, and end of treatment/survivorship). For this reason the authors represent the coping process in the form of a wheel (Figure 2), to underline the constant adjustment required to the dyad (Kayser \& Scott, 2008).

The first step of the coping process is the stress appraisal, as the cancer diagnosis can be considered as a source of stress that affects both the patient and the dyad. When this 
occurs, partners tend to engage in a stress communication which has the potential to offer a form of validation to the members of the couple. After appraisal and response, the third step of the model refers to the coping behaviors of the partners. When partners are able to coordinate problem and emotion-focused coping behaviors, then the outcome is relational and individual growth (Kayser \& Scott, 2008).

Within this framework, different appraisals and responses to cancer are shaped by relational characteristics. Relationship awareness, authenticity and mutuality are identified by the authors as the relationship qualities that will determine the pattern of coping couples will develop, and for this reason they are symbolically placed in the center of the hub. Relationship awareness refers to the partners' awareness that the stressor, namely the illness, is affecting both partners in their relationship (Kayser et al., 2007; Kayser \& Scott, 2008). Authenticity describes partners' ability to disclose genuine feelings to each other in a sensitive and appropriate way (Kayser et al., 2007; Kayser \& Scott, 2008; Kayser \& Scott, 2009b). Finally, mutuality is defined as the ability to be empathic with the partner and to participate in a shared experience (Feldman \& Broussard, 2005; Feldman \& Broussard, 2006; Jordan, 1997; Kayser et al., 2007; Kayser \& Scott, 2008). In a close relationship, mutuality is conceptualized as the "bidirectional movement of feelings, thoughts, and activity between persons in relationship" (Genero, Baker-Miller, \& Surrey, 1990, p.1). While it is often confused with social exchange, by assuming a relational perspective, mutuality becomes a set of actions that encompasses "diverse modes of social interaction that facilitate participation in and growth through relationships" (Genero et al., 1992, p. 36). 
Depending on the presence of these characteristics, two different patterns of relational coping are enacted: mutual responsiveness and disengaged avoidance (Kayser et al., 2007, Kayser \& Scott, 2008). Couples characterized by mutual responsiveness appraise the stress of cancer as affecting both members of the couple and they initiate a stress communication process that leads to mutually coordinated coping behaviors (Kayser et al., 2007; Kayser et al., 2011). It is important to clarify that these couples do not necessarily report the same issues or complaints. On the contrary, they are able to tolerate differences in their experience with cancer. As partners are able to listen and validate the stress of each other, their coping behaviors become coordinated (Kayser \& Scott, 2008). Mutually responsive couples are in fact able to implement either problemfocused or emotion-focused strategies, which promote a sense of growth as individuals and as a couple. On the contrary, a disengaged avoidant pattern of coping is characterized by the persistent appraisal of cancer as an individual stressor, and partners' communication lacks expression of emotions and feelings. Other couples are not able to identify that a change has, indeed, happened or, on the contrary, to recover from the sense of loss experienced. Hence, at least one partner copes by avoiding or denying the effect of cancer or the coping strategies are limited to problem-solving, with a cascade effect on other significant relationships. The outcome is that partners cannot find any benefit from the experience (Kayser et al. 2007; Kayser \& Scott, 2008; Kayser et al., 2011). 
Figure 2. Relational-Cultural Coping Process

(Edited from Kayser \& Scott, 2008)

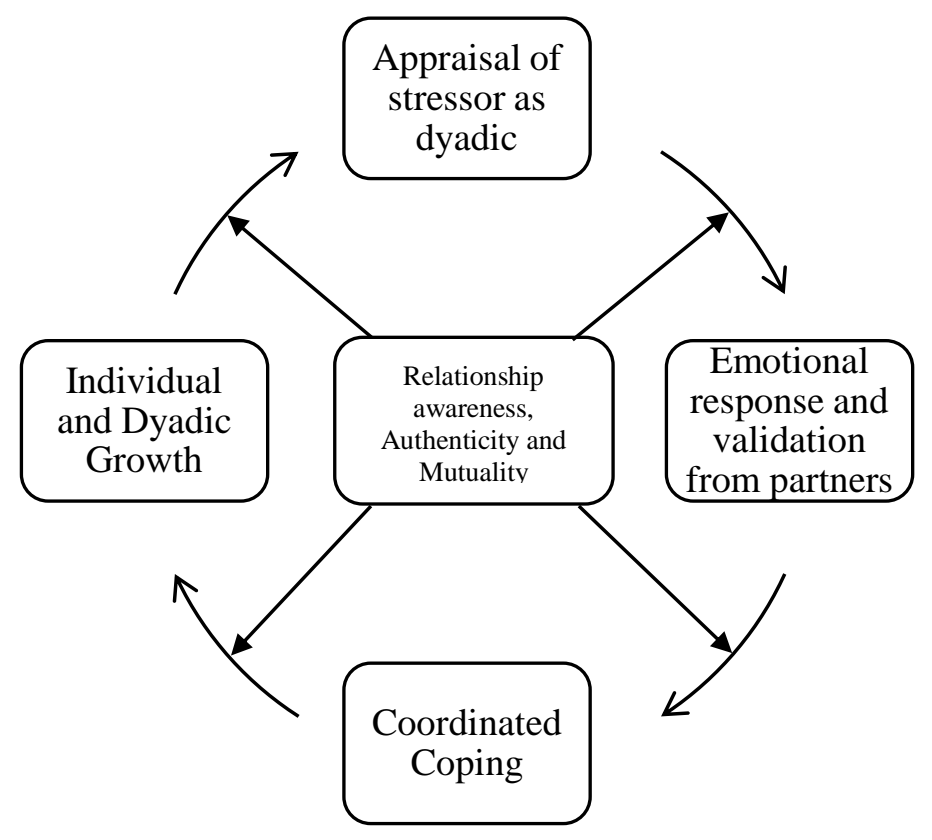

Influenced by the Systemic-Transactional Model of dyadic coping (Bodenmann 1995; 2005), and by the Stress and Coping Theory (Lazarus \& Folkman, 1984), a unique aspect of this conceptualization is the reliance on the Relational-Cultural Theory, a perspective that explains the individual sense of self as being in relation (Jordan, Kaplan, Miller, Stiver, \& Surrey, 1991; Miller, 1984). According to this framework, the goal of human development is the acquisition of relational competence, which can be achieved by engaging in growth-fostering relationships. The theory has been used in previous works (Kayser, et al., 1999; Bekteshi \& Kayser, 2013) investigating breast cancer patients' adaptation to illness and the impact of cancer on the relationship between mothers and daughters. Results from these studies highlight that coping abilities are deeply influenced by the quality of ongoing close relationships (Kayser et al., 1999). When the researchers have investigated dyads of mothers and daughters, qualitative 
analyses have shown that relational growth in the cancer aftermath was associated with interpersonal skills like anticipatory support, authenticity, mutual empathy and empowerment (Bekteshi \& Kayser, 2013). Therefore, it follows that the model places the ability to cope with cancer-related stress within the relational competencies of the dyad, and not just as the result of individual coping strategies.

However, this conceptualization presents some limitations. First, as its use in the literature is limited, these initial results have not being confirmed in quantitative studies and a revision of the Stress and Coping Theory that includes couple relational qualities is in process but not yet completed (Kayser \& Scott, 2009b; Saita, 2011). Second, the model does not address the importance of developmental and contextual aspects; which have been investigated in more recent works (Berg \& Upchurch, 2007).

\section{Developmental-Contextual Model}

The most recent contribution to the theoretical reflection about dyadic coping is the Developmental-Contextual Model (Berg \& Upchurch, 2007). Starting from a critique of previous conceptualizations, Berg and Upchurch introduced the idea that dyadic coping changes across the adult life-span, historical times, daily interactions with dyadic stressors and it is influenced by the context (Berg \& Upchurch, 2007). Authors emphasize that appraisal and adjustment can be influenced by normative modifications in self-development of the two partners, their emotion regulation abilities, and marital processes. All of these may vary as a result of sociological and historical events. In addition, appraisal and coping efforts take place over time as the couple moves along the different stages of the chronic illness (Berg \& Upchurch, 2007; Revenson \& DeLongis, 2011). 
Developmental differences are anticipated between younger and older couples based on the literature that indicates the presence of higher satisfaction and intimacy in long-term marriages (Carstensen, Graff, Levenson \& Gottman, 1996; Lauer, Lauer, \& Kerr, 1990). Better competence in the ability to regulate emotions and appraisal of stress are also reported in older individuals, which tend to show greater mutuality and less maladaptive coping (Aldwin, 1994; Diehl, Coyle, \& Labouvie-Vief, 1996; Folkman, Lazarus, Pimley \& Novacek, 1987; Labouvie-Vief, 2003). The second developmental aspect is the temporal process of dyadic coping. In their reflection the authors cite results from studies that have highlighted the changing nature of dyadic coping over time, especially in the case of an illness (Fang, Manne, \& Pape, 2001; Helgeson, Snyder \& Seltman, 2004; Martire, Stephens, Druley, \& Wojno, 2002; Schulz \& Schwarzer, 2004). Again, age-related differences have been identified because younger individuals report greater distress and reduced ability to perform collaborative coping (Helgeson et al., 2004; Revenson \& Pranikoff, 2005). In contrast, older adults become better able to cope effectively (Revenson, 2003). Finally, among the contextual variables that affect interdependent appraisal and dyadic coping it is possible to list cultural and gender differences, the quality of the marital relationship, and the type of chronic illness (Berg \& Upchurch, 2007).

Consistent with the social contextual perspective (Rogoff, 1998; Vygotsky, 1987), coping strategies are viewed in relation to those enacted by the other partner, and vice versa. In their approach to dyadic coping Berg and Upchurch (2007) examine illness representations, illness ownership and stressor appraisal of both members of the dyad. Four configurations of dyadic coping are identified, ranging from un-involvement to 
over-involvement (Berg, Meegan, \& Deviney, 1998; Meegan \& Berg, 2002). The construct of collaborative coping is defined as "the active engagement of spouses in pooling resources and in joint problem-solving and coping” (Berg et al., 2008, p. 506) ${ }^{7}$. Collaborative strategies are characterized by partner's equal involvement and shared decision-making (Meegan \& Berg, 2002); in contrast to uninvolved ${ }^{8}$, supportive $^{9}$, and control strategies ${ }^{10}$. Collaborative coping serves two major functions: resource and relationship enhancement (Iafrate \& Donato, 2012).

In recent years the model has started to be applied to the study of couples' experience with cancer. Collaborative coping was associated with positive mood not only for patients coping with prostate cancer, but also for their partners. This relationship was mediated by patients' and partners' effectiveness of coping; a result that suggests that the perceptions to engage in behaviors that promote the couple relationship may be as relevant as the actual behaviors (Berg et al., 2008; Regan et al., 2015a). Changes in physical health of the patient and depressive symptoms in couples coping with lung cancer were examined by Lyons et al. (2014). The interdependence of the participants' well-being over time was confirmed: pain severity was significantly associated with depression in both partners, while physical function of patients was negatively associated with depressive symptoms (Lyons et al., 2014). More recently, a cross-sectional study by Magsamen-Conrad, Checton, Venetis and Greene (2015) examined communication efficacy and couple's cancer management. In this group patients' confidence in the

\footnotetext{
${ }^{7}$ This definition of collaborative dyadic coping presents similarities with the concept of common dyadic coping introduced by Bodenmann (2005), yet the authors haven't addressed how collaborative coping diverges from previous conceptualization of dyadic coping and from similar coping styles presented in the literature.

${ }^{8}$ Individual coping strategies in which partners act on their own.

${ }^{9}$ Instrumental or emotional supportive responses.

${ }^{10}$ Coping behaviors characterized byover-involvement and behavior control.
} 
ability to communicate about cancer predicted their ability to manage their needs, while for partners their confidence predicted their own and their patients' ability to cope with the illness.

Although these results provide initial support for the model, it is necessary to comment on the fact that none of the publications currently available in the literature have compared the experience of couples across the life trajectory, nor cohort or historical/contextual variables have been included in the analysis ${ }^{11}$. Also, the authors appear to have created the model "ad hoc" integrating findings from the literature and other conceptualizations, while a practical application of the model appears to be difficult. Further research is needed to understand how couples' appraisal, coping and adjustment are shaped by developmental, temporal and contextual factors.

\footnotetext{
${ }^{11}$ Referring to the articles identified by Regan et al. (2015a) as well as to a independent search, it is possible to identify that four articles currently have applied the Developmental- Contextual Model: Checton, Magsamen-Conrad, Venetis, and Greene (2015); Berg et al., (2008); Lyons et al., (2014), and Magsament-Conrad, Checton, Venetis, \& Greene (2015). Checton et al. (2015) measured support as proxy for dyadic coping; Berg et al. (2008) utilized a non-standardized 12-item questionnaire developed by the first author; Lyons et al (2014) did not includ coping in their design; while Magsamen-Conrad et al. (2015) included communication efficacy as a proxy for patient and partner coping. No indication is provided about the inclusion of age in the statistical analysis.
} 


\section{Toward a Conceptualization of Dyadic Coping to study Couples' Adjustment to}

\section{Cancer}

In the process to identify an appropriate conceptualization of dyadic coping to study couple's adjustment to cancer, a researcher is then confronted with the number of models currently available in the literature. Although each of them emphasizes different features, they share some conceptual similarities. First, they all converge on the fact that dyadic coping involves the reciprocal engagement in strategies to support the coping effort of the partners. Hence, both members of the couple are involved in conjoint coping processes as the result of a shared appraisal. Second, these behaviours - which can range from active engagement to disclosure of emotions- contribute to increased communication, higher satisfaction, and reduced distress. Finally, another similarity is that negative style of dyadic coping, avoidance and protective buffering have a detrimental effect on the individual's well-being and the couple relationship (Regan et al., 2015a; Traa et al., 2015b).

However, some of the models fail to address the relevance of the relationship and its characteristics in determining the type of appraisal, coping strategies and outcomes associated with experiencing the stressful event. Recent data support the use of a systemic model of dyadic coping versus a congruent/discrepant approach (Bodenmann et al., 2011; Regan et al., 2015a). Bodenmann, Meuwly and Kayser (2011) compared the congruence model proposed by Barbarin et al. (1985) and Revenson (1994; 2003), with a systemic model of dyadic coping (Systemic-Transactional Model). Results indicate that the latter is a stronger predictor of relationship quality and health outcomes (Bodenmann et al., 2011). More recently, Regan et al. (2015a), have tried to identify the main 
theoretical frameworks in the context of dyadic coping and cancer. Based on their results, the authors affirm that models able to "incorporate the interdependence of emotional responses and coping behaviors" (ibidem, p.1) present better support. Despite the scarcity of this type of studies, it is possible to affirm that the different models represent also an evolution in the understanding of dyadic coping, and that over time conceptualizations that are truly relational and dyadic are emerging in the literature.

The model proposed by Kayser et al. (2007) appears to be appropriate to address couple's coping with cancer for three reasons. First, it integrates significant contributions of previous models while maintaining its unique relational perspective. For example, clear are the influences from the Lazarus and Folkman (1984) model of stress and coping and Bodenmann's theory of dyadic coping as systemic-transactional (Bodenmann, 1995; 1997). Similarly, this conceptualization validates emotional and problem-solving strategies. Moreover, the model appears to be able to capture the relevance of the relationship in shaping coping efforts. In this sense, the model places the ability to cope with cancer as a product of the relational characteristics of the couple, not the individual. It is critical that the couple is able to coordinate their resources, not that both members have those specific resources. On the contrary, other models and conceptualizations have been focusing on the individual coping strategy, without acknowledging that those strategies are placed within a story and a context. Furthermore, the authors place dyadic coping within the cultural context, highlighting that relational qualities and coping approaches are "embedded in culture" (Jordan, 2009). In this sense the model is able to include the recommendation of Revenson (Revenson et al., 2005; Revenson \& DeLongis, 2011) and Berg and Upchurch (2007) about the relevance of the environment and 
addresses the power of socioeconomic status, gender and race in influencing couples' experience. Finally, the model is also the only one developed from a sample of couples coping with cancer and to identify different and unique challenges associated with the course of the illness. The other models have been developed with the goal to examine dyads coping with chronic illnesses and everyday stressors. Couples coping with cancer experience a unique set of stressors (medical, instrumental, social, emotional and existential) that are significantly different from the ones of everyday life (Kayser, 2005). Hence, a model that focuses on the specific demands and needs the illness originates in the couple appears to be promising. The Relational-Cultural Model represents an encouraging direction for future work as it is able to integrate temporal and contextual dimension, next to the focus on the role of relationships in determining individual's wellbeing and growth. In the following paragraph the conceptual framework of the study is presented, and the theoretical framework that supports this model is reviewed in the second part of the chapter.

\section{Conceptual Framework}

A strong body of evidence has been collected about the role dyadic coping has in promoting quality of life of both members of the dyad. However, despite the extensive knowledge about couples coping with illness developed in the last twenty years, several gaps can be identified in the literature. First of all, a clear understanding of the mechanism through which dyadic coping originates and contributes to both relational and individual outcomes is still elusive. Second, the attention to the developmental trajectory of dyadic coping over the life-span is a significant gap in the current literature and a paucity of studies have specifically investigated these areas. The present research study 
therefore is aimed at examining how younger couples cope with breast cancer, by comparing the experience of couples at different developmental moments. By doing that, the study tests the Relational-Cultural Model of dyadic coping (Kayser et al., 2007). According to this conceptualization, appraisals and responses to cancer originates from relational characteristics of the partners. In particular, mutuality is defined as the ability to be empathic with the partner and to participate in a shared experience (Jordan, 1997; Kayser et al., 2007; Kayser \& Scott, 2008). The presence of this relational ability is associated in this conceptual framework to coordinated forms of dyadic coping, which ultimately lead to individual's quality of life (Figure 3). Hence, it is hypothesized that dyadic coping mediates the relationship between mutuality and quality of life of each member of the dyad, with positive forms of dyadic coping contributing to higher quality of life, while maladaptive coping strategies are expected to affect the beneficial impact of mutuality on well-being ${ }^{12}$. Initially, it was planned to control for socio-demographic variables, in particular age and length of relationship. However, given the small sample size and the requirements of the statistical analyses implemented, it was not possible to adhere to the original plan and for this reason this indication is not shown in the figure.

\footnotetext{
${ }^{12}$ Since this was the first empirical testing for the model it was preferred to formulate non-directional hypotheses to explore the relationship among these constructs.
} 
Figure 3. Conceptual Framework of the study

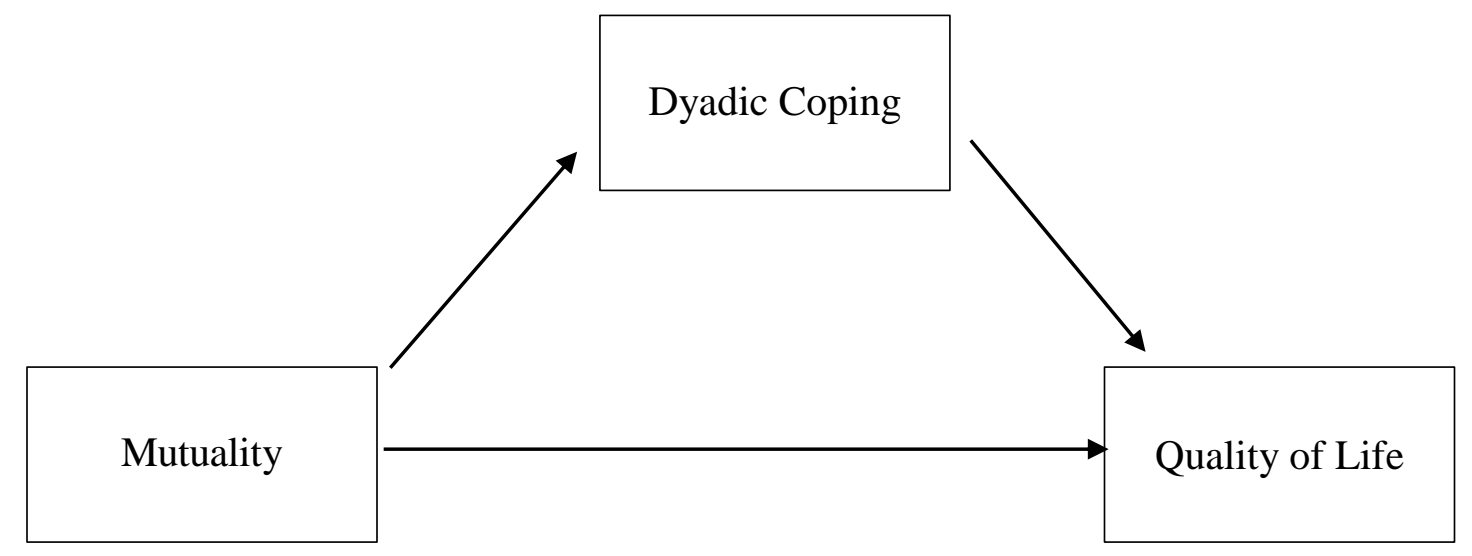

\section{Systems Theory}

\section{Review of Systems Theory}

Contemporary theories about family functioning and models of family resilience derive from General System Theory (GST) (Von Bertalanffy, 1969). It is organized around the concept of system, whose definition as a "set[s] of elements standing in interrelation among themselves and with the environment" (Von Bertalanffy, 1975; p. 159) already summarizes some key features. First, the theory states that all elements of a system are interconnected (interdependence) and that the behavior of each component is characterized by mutual influence (White \& Klein, 2008). Another core assumption is that of holism: a system can only be understood as a whole and cannot be comprehended by analyzing its composing parts in isolation from each other (wholeness or nonsummativity) (Whitchurch \& Constantine, 1993; White \& Klein, 2008). Human systems are self-reflexive. Self-reflexivity allows human beings to examine themselves, their behavior and establish goals. Communication is what allows self-reflexivity to occur because it facilitates the creation of meaning and the simultaneous exchange of symbolic 
content (Whitchurch \& Constantine, 1993). Despite a natural tendency to reach and to maintain a balance (homeostasis), a system constantly exchanges and interacts with its environment and restructures its internal functioning to more effectively answer to the changes in the environment (morphogenesis) (Whitchurch \& Constantine, 1993; Gurman \& Kniskern; 2003). As the concept of boundary defines who is included and who is not, systems are usually classified as open or closed. However, boundaries may have different permeability which limits the amount of information in and out of the system (Whitchurch \& Constantine, 1993). Finally, systems are characterized by internal rules of transformation and variety. Rules of transformation describe the relationship existing between two elements of the system while variety indicates the resources available to the system to adapt to changes or to meet the demands of the environment (White \& Klein, 2008). Systems with more variety are more able to adapt to a constantly changing environment, while for systems with limited variety adaptation to changes appear to be more rigid (White \& Klein, 2008).

\section{Relevance of Systems Theory for the study of Dyadic Coping}

Systems Theory contributes to our understanding of dyadic coping by highlighting the fact that both partners are affected by the cancer experience, and their reactions are actually interdependent. As reviewed in the first part of the chapter, several studies have confirmed that the well-being of patients and partners is associated with the reaction of the other member of the dyad. It is then possible to affirm that partners act as an emotional system. As a consequence, by conceptualizing the couple as a unit that influences the functioning of each person (Bowen, 1978), Systems Theory also indicates that the couple system can only be understood as a whole. This has far-reaching 
consequences for researchers interested in studying dyadic coping, since it implies that couple assessment and measure of dyadic coping have to be conducted on the dyad rather than on individuals. Third, Systems Theory indicates that a system's behavior affects the environment and vice versa. This is critical for dyadic coping, because the theory already highlights that the couple is influenced by the larger social environment. Dyadic coping is then understood as a product not only of partner's characteristics and their relationship, but also of the influence of outside forces. Similarly, the concept of boundary is helpful to explain the relation between the couple and the environment. Because there are not completely closed or open systems, every dyad has different "degree of permeability" (White \& Klein, 2002; 2008) to the influences of the outside.

\section{Limitations}

Despite the strengths highlighted earlier, Systems Theory has some limitations. The theory has been criticized for being an abstraction because concepts can be difficult to operationalize for research purposes. It is then considered more a philosophical perspective, as it provides universal explanations and a general understanding of family and couple interactions (Bowen, 1978), but not the details that are necessary for empirical testing (White \& Klein, 2002; 2008; Whitchurch \& Constantine, 1993).

\section{Transactional Theory of Stress and Coping}

\section{Review of the Transactional Theory of Stress and Coping}

The Transactional Theory of Stress and Coping, originally developed by Lazarus and Folkman (1984), has been extensively used to investigate the individual adaptation to stressful events for more than thirty years (Folkman \& Moskowitz, 2004; Folkman, 2011; 
Stanton, Revenson, \& Tennen, 2007). The theory integrates Behaviorism (Dember, 1974; Lazarus, 1966) and Cognitive Theory (Beck, Rush, Shaw, \& Emery 1979; Ellis, 1957) to examine personal, environmental and situational factors as antecedents of psychological outcomes.

Different from other authors, Lazarus's view of stress was not associated with a single event, but rather it was the result of a transaction (process) between the individual and the environment. While Cannon's "fight or flight" responses to stress (Cannon, 1932), and the General Adaptation Syndrome research by Selye (1956) focused on the individual's automatic reaction to the environment, the theory developed by Lazarus and Folkman moved the attention to psychological factors and the concept of appraisal. Therefore, the most emphasized assumption is that stress results from the individual's response to the event (Lazarus, 1990; 1993; Lazarus \& Folkman, 1984). Hence, the impact of an external stressor is mediated by people's appraisal, which then influences the emotional arousal (Folkman \& Lazarus, 1988). In this sense, it is possible to affirm that appraisal represents "the primary mediator of the person-environment transactions" (Lyon, 2012; p. 9). The authors identify three types of appraisals: primary, secondary and reappraisal. When confronted with a stressor, individuals evaluate the threat, loss or challenge posed by the situation (primary appraisal) as well as the resources available to cope with it (secondary appraisal). The event is then considered stressful based on the individual perception of availability of resources. Appraisal is a complex evaluative process, as the introduction of the concept of reappraisal indicates. People continually evaluate, change or re-label primary and secondary appraisals while the situation evolves over time (Lazarus \& Folkman, 1984). 
Within the cognitive-transactional model, coping is defined as the "constantly changing cognitive and behavioral efforts used to manage specific external and/or internal demands that exceed the resources of the individual" (Lazarus \& Folkman, 1984; p.141). Two forms of coping have been identified by the authors and in the subsequent literature: problem-focused coping and emotion-focused coping ${ }^{13}$. While in its original formulation coping was considered a personality trait, more recent contributions define coping as a process that changes over time and is influenced by situational factors. It is now considered a multidimensional construct, where personal, contextual and social factors are critical (Alberisio e Viterbori, 2002; Solano, 2001; Zani e Cicognani, 2002).

\section{Relevance of the Stress and Coping Theory for the study of Dyadic Coping}

The Stress and Coping Theory has provided a strong conceptual base to evaluate and understand the individual's adaptation to stressful events, becoming the theory of reference for researchers interested in coping. It is therefore necessary to reflect on its contribution to our understanding of dyadic coping.

First, the model places strong emphasis on the importance of appraisal. According to Stress and Coping Theory, individuals' emotional and behavioral responses to health threats are influenced by subjective interpretations, which are cognitive and emotional. In this sense, when focusing on dyadic coping couples evaluate the stress they have to face and that they can perceive it as impacting both members of the couples or only the individual. If the members of the couple perceive the stress as affecting the both of them,

\footnotetext{
${ }^{13}$ Emotion-focused coping strategies are aimed at reducing the emotional responses caused by the event and include wishful thinking, minimization, avoidance, or denial. On the contrary, problem-focused coping is mainly used to alter the stressor by action, using problem-solving skills (information seeking, evaluating the pros and cons, taking control). Problem-focused coping strategies are most adaptive for stressors that are changeable, whereas emotion-focused strategies are appropriate when the stressor is appraised as uncontrollable or when problem-focused attempts have been exhausted (Folkman \& Greer, 2000; Lazarus \& Folkman, 1984; Wenzel, Glanz, \& Lerman, 2002).
} 
then they will engage in a process of conjoint coping. As our understanding of the process that leads to dyadic coping is still limited, this process is unclear. However, the core element from the Lazarus and Folkman model (1984) is the idea of coping as a transaction between the two partners, and between the couple and the environment. Hence, dyadic coping takes place within the couple relationship and within the multiple social systems the partners are part of.

Another critical aspect is the fact that dyadic coping must be studied as a process, as it changes over time. Research has shown that couples often face different challenges at different phases of the illness (i.e., before diagnosis, during and after treatment, and approaching survivorship), which forces them to master different coping skills (Gray et al., 2000). Thus, the Stress and Coping Model provides a theoretical basis for studying the dynamic coping process over time. Finally, it supports our understanding of the coping strategies used by couples, as the distinction between problem-focused and emotion-focused coping strategies has been applied also to dyadic forms of coping.

\section{Limitations}

Despite its relevance, the Stress and Coping Theory has some limitations that impact its use in studying the experience of couples coping with cancer. First, the model has been developed to explain individual's reaction to general stress. It therefore fails to address the specific aspects of a stressor that affects the dyad. Second, despite the theory highlights the relationship between the individual and the environment, little attention has been given to the context. Third, the most relevant limitation is its highly individualistic perspective which focuses on the relationship between stress, appraisal and coping and minimizes the influence that others may have on adjustment process. Individuals are 
perceived as functioning independently, without addressing the role of significant relationships for human development and well-being. The model states that individuals independently choose coping strategies and devote coping efforts to manage the stressor (Lazarus \& Folkman, 1984; Wenzel, et al., 2002). Such an individualistic perspective has concealed individuals' need for relationship and for interdependence, and thus, has given insufficient attention to the importance of other social, situational and environmental factors (Lyons, Mickelson, Sullivan, \& Coyne, 1998; Iafrate \& Donato, 2012). This limitation is particularly relevant when focusing on couple's adjustment to illness, since it has been demonstrated that cancer patients and their partners are interdependent with one another, and function as system (Hagedoorn et al., 2008).

However, this theoretical perspective is so central to the understanding of dyadic coping that researchers have tried to modify it rather than developing a new one. For example, Northouse, Kershaw, Mood, and Shaughenacker (2005) modified the Transactional Model of Stress and Coping to study coping efforts and outcomes of both cancer patients and family caregivers. Two major changes were introduced. First, Systems Theory was integrated to reflect the interdependence in coping responses. Second, illness-related factors that may influence how patients and their family members appraise and cope with an illness were included. Similarly, Scott \& Kayser highlighted (2009b) that the Transactional Theory of Stress and Coping should be reviewed to examine the role of meaning-based coping. They proposed a revision of the model able to capture the relational qualities of the couple and the connection between dyadic coping, individual coping and relational satisfaction. While these efforts are still ongoing, the theory maintains a central role in our understanding of couples coping. 


\section{Relational-Cultural Theory \\ Review of Relational-Cultural Theory}

Relational-Cultural Theory views connections as the primary organizer and source of motivation for the individual (Jordan, 2009). A reaction to developmental and clinical theories organized around the importance of the separate self -which praises autonomy, self-boundaries, and individuation (Jordan, Kaplan, Miller, Stiver, \& Surrey, 1991)- the theory states that human development is based on the ability to develop connections and being interdependent (Jordan, 2009). Core concepts are that human beings grow through and toward relationships across the whole life-span and that mature functioning is characterized by mutuality (Comstrock, Hammer, Strentzsch, Cannon, Parsons, \& Salazar, 2008; Freedberg, 2015; Jordan, 2000; 2009; Jordan \& Hartling, 2002). This movement toward mutuality is essential for successful coping and healthy development, because in relationships characterized by mutuality individuals are genuinely interested in the other as a whole, complex person and are aware of the other's subjective experience (Jordan, 1991).

Theorists assuming this perspective believe that the goal of development is the creation of increased relational competence, which can be achieved by engaging in growth-fostering relationships characterized by empathy and authenticity (Comstrock et al., 2008; Freedberg, 2015; Jordan \& Hartling, 2002). Empathy is defined as a cognitive and affective capacity for "resonance and responsiveness to others" (Jordan, 2009, p. 4) and mutual empathy is the process that allows for growth in relationships, as individuals feel better able to to participate in the shared experience (Jordan, 1997). Authenticity is defined as the ability to disclose feelings (Spencer, 2005). According to Jean Baker 
Miller (Miller, 1976; 1989; Miller \& Stiver, 1997) growth-fostering relationships have five outcomes: a sense of energy, better understanding of the self, increased capacity for action, increased sense of worth and desire for connection (Jordan \& Hartling, 2002; Miller, 1976). Problematic on the contrary are disconnections that cannot be addressed and reworked which lead to rigid relational images. These are internal constructions and expectations originated earlier in life (Miller \& Stiver, 1997). When they are fixed and cannot be changed, the individual experiences a condition of isolation (Miller, 1989). However, healing can occur in the context of growth-fostering relationships (Comstock et al., 2008).

A second specific characteristic is its being "cultural". Relational-Cultural Theory recognizes that marginalization, privilege and cultural forces are central for the psychological development (Comstock et al., 2008). In this sense, culture is an active element that shapes relational processes, as relationships take place in contexts influenced by factors like race, gender, class, physical ability and religion (Walker, 2005). This attention to increasingly complex cultural, social and relational environments mirrors the evolution of the theory. Relational-Cultural Theory was initially developed within a feminist framework, which criticizes the way traditional theories have misrepresented women's experiences. Inspired by the seminal work of Carol Gilligan (1982), who highlighted that women make decisions based on sense of responsibility and care, Miller developed the first version of the theory. In her ground-breaking book (1976) she argued that role, function and social structuring of gender are connected with understanding women's identity development. As a consequence, the "self-in-relation" theory emphasized the fact that for women "the core sense of self is organized, developed and 
maintained in the context of important relationships through life" (Surrey, 1991 cited in Freedberg, 2009, pg. 24). In the early 1990s the theory was renamed the RelationalCultural model to emphasize relational processes among individual and contemporary factors that impact relationships (gender, class, ethnicity and sexual preference) and contribute to a politics of dominance (Miller \& Stiver, 1997).

\section{Relevance of the Relational-Cultural Theory for the study of Dyadic Coping}

While previous theories contribute to the definition of dyadic coping, RelationalCultural Theory (Jordan, 2009) is essential to understand the function of dyadic coping. Through these theoretical lenses, dyadic coping is aimed at protecting patients and partners' ability to be in a relationship with a significant one. At the same time, the theory highlights the importance of mutuality, authenticity and empathy. In this sense dyadic coping enhances the development of the individual by engaging in growth-promoting relationships. It is particularly interesting to think that dyadic coping represents not only a reaction to the stressful event, but that through the protection of the relationship with the other it contributes to define, preserve, and promote the individual's sense of Self.

The theory contributes to understand adaptive or maladaptive coping strategies, as they are influenced by the nature of the relationship. Relational-Cultural Theory states that when "one person misunderstands, invalidates, excludes, or humiliates" the other (Jordan, 2009, p. 25), then partners will learn to suppress their needs or will engage in a series of attempts to try to fit in (Comstrock et al., 2008: Freedberg, 2015; Jordan, 2009). This then creates a condition of inauthenticity, isolation and disempowerment. Maladaptive coping strategies characterize couples where a partner is perceived unavailable to accept and support the needs of the other. Furthermore, the theory attempts 
to identify culture as an active agent in relational processes (Freedberg, 2015; Walker, 2005). Hence, dyadic coping takes place within the larger social context and under the influence of factors like race, class, and gender.

Finally, Relational-Cultural Theory includes aspects of the work of Melanie Klein and Karen Horney, next to interpersonal psychoanalysis (Sullivan, 1940), object relations (Bowlby, 1988; Fairbain, 1952, Winnicott, 1960), and self-psychology (Kohut, 1978). These approaches, which have found more fertile ground in the European literature, emphasize the importance of the individual's sense of Self and the interactions between the individual and the object, highlighting that nature and quality of the interaction with others ultimately determine the individual's well-being (Freedberg, 2009). More recently, support for the relevance of human connection for the individual's well-being has been found in interpersonal neurobiology and Social Brain Theory, since for both Siegel and Cozolino human connections shape the structure and function of the brain (Cozolino, 2006; Siegel, 2012).

\section{Limitations}

One possible critique to the use of this theory as theoretical foundation for the study of dyadic coping is that the Relational-Cultural Theory was initially developed from a feminist perspective. However, it has undergone several revisions in the last thirty years and it now represents a psychological theory applicable to both men and women. The recognition of differences among the developmental experiences of the two genders is actually helpful, as we have seen that men and women report different coping mechanisms in response to the cancer experience, as well as diverse reactions to the partner's coping behavior. In this sense, different coping strategies will be seen as the 
result of different developmental processes: through attachment and affiliation for women and through autonomy and separation for men (Gilligan, 1982; Miller, 1976). A second limitation of the present theory is its utilization in Social Work, while it was originally developed in Psychology. However, it appears fully compatible with the principles and values of the discipline. Not only it provides a contextual relational view of the Self consistent with the person-in-environment view of the field, but it is also in line with the ethical principles of importance of relationship, dignity and worth of person, and social justice (Freeman, 2000).

\section{Life Course Developmental Framework}

\section{Review of the Life Course Developmental Framework}

The Life Course Developmental Framework originates from the integration of three complementary theoretical approaches: Individual Life Span Theory, Family Development Theory, and Life Course Theory (White \& Klein, 2008; White, Klein \& Martin, 2015). Although each theory's contribution is unique, they have been combined in a single framework because of existing similarities. First, they focus on family factors that can affect the ontogenetic development of the individual. Second, all three theories consider the effect of time on individual and group change. Finally, individual and family change is considered to be part of the larger framework of birth cohort, historical period, and individual age factors (White \& Klein, 2008; White et al., 2015).

Individual Life Span Theory is organized around the study of the ontogenetic development of the individual and of the factors that affect it (White \& Klein, 2008: White et al., 2015), attempting to extend models developed for children development to 
adults. Introduced in the early ' 60 s, Family Development Theory focus on the concept of stage (Duvall, 1957; Duvall \& Hill, 1948; Smith \& Hamon, 2012; White, 1991). Stages are periods of "relative equilibrium in which consensus about the allocation of roles and rules of procedure is high" (Hill, 1986, p. 21). Qualitatively different from each other, the passage from a stage to another is associated to normative events like marriage, childbirth, developmental and educational milestones. Families undergo change and development through time, both in terms of roles of the members and stages, which occur through transitions ${ }^{14}$ (Hagestad, 1988). Once considered similar and predictable over the life course, transitions from one stage to another have now more variation among family systems (Laszloffy, 2002). Tasks are associated with each stage of development, and the inability to move across stages may limit the family's optimal level of functioning. Family's behavior is regulated by institutional norms that control "which events are permitted, required, and forbidden; the order in which families should sequence stages; and the duration of those stages" (White, 1991, p.57).

For the purpose of the present work, the last theory included in the framework assumes a significant relevance. In its original version, Life Course Theory integrates psychological developmental theories and sociology to identify agents of change across the life of the individuals (Elder, Johnson \& Crosnoe, 2006). It proposes that in order to understand peoples' growth and adaptation to major life events, the focus of analysis

\footnotetext{
${ }^{14}$ The original eight-stage model includes married couple, childbearing, preschool and school age, teenage years, middle aged parents and aging family members (Duvall \& Miller, 1985). More recently, Laszloffy (2002) proposed a new model that integrates Family Systems Theory, Family Stress Theory and a multigenerational perspective to support that the developmental pattern of each family is unique. From her perspective each family can be described as a cake, where each layer represents a generation experiencing a different stage of development. As a consequence, the investigator has to study the relations between the layers and the challenges associated with each new stressor the family encounters to account for between and within variations (Smith \& Hamon, 2012).
} 
should be the lived experiences of individuals in contexts (Elder, 1998; 2000; 2002). According to this theoretical perspective, events are combined in trajectories that are compared across persons or groups, focusing on differences in timing, duration and rates of change within a given social milieu (Elder, 1998:2000; 2002; Elder \& Shanahan, 2006). Social norms or shared expectations about the timing of events and role transitions will dictate the classification as either "off-time" (unexpected) or "on-time" (expected) events (Hutchison, 2008). Life Course Theory includes five paradigmatic principles: 1) human development and aging are lifelong processes; 2) individuals build their own life course through choices and actions that are influenced by historical and social circumstances; 3) the life course is embedded by historical context and place; 4) the meaning of life transitions, events and behavioral patterns can change based on their timing in the life experience of the individual; and 5) socio-historical influences can manifest themselves through the impact on interdependent relationships. These principles promote awareness of the larger social context and history and about the different timing of events and role change associated with a specific cohort (Elder, Johnson \& Crosnoe, 2006; Elder \& Shanahan, 2006). Furthermore, the life course perspective has been successfully applied to the study of families (Smith \& Hamon, 2012; White et al., 2015).

\section{Relevance of Life Course Developmental Framework for the study of Younger Couples}

\section{Coping with Cancer}

A limited number of studies have explicitly examined age differences in coping during adulthood and trajectories of dyadic coping over the life course (Folkman, 2011; Revenson \& Lepore, 2012). However, a developmental approach offers multiple elements of reflection for the study of couples coping in cancer. 
First, if we consider the development of coping abilities through the life-span, evidence from the literature presents mixed results. While some studies show no differences, others suggest that an individual's coping competences increase with age (Aldwin, 2010; Aldwin, Skinner, Taylor, \& Zimmer-Gembeck, 2011; Blanchard-Fields, Sulsky, \& Robison -Whelen, 1991; Felton \& Revenson, 1987; Irion \& Blanchard-Fields, 1987; Verhaeghen \& Hertzog, 2014). In one of the first cohort studies, younger individuals reported more confrontative coping and social support than the older cohort. The younger cohort also experienced higher levels of daily stressors in the domains of finance, work, personal life and family relations (Folkman, Lazarus, Pimley, \& Novacek 1987). Over time older adults use fewer hostile and avoidant strategies (Aldwin, 1991; 2010; 2011; Aldwin, Sutton, \& Lachman, 1996; McCrae, 1989), less rumination, emotional numbing, and wishful thinking (Wadsworth et al., 2004). Both cross-sectional and longitudinal studies suggest that the use of mature defense mechanisms increases with age (Bond et al., 1983; Diehl et al., 1996; Vaillant, 1977; 1993). It then appears likely that coping changes with age during adulthood and that, with experience, individuals develop better emotion regulation and more effective collaborative coping skills (Aldwin et al., 1996; Aldwin, 2010; Rothermund \& Brandstadter, 2003).

Second, coping may be more difficult for younger couples because of the stage in their family development and for the specific sources of stress experienced by Millennials (18-35 y.o.) and generation Xers (36-49 y.o.). As described by the American Psychological Association (2015), younger generations of Americans report higher levels of stress, often associated to financial insecurity and un-healthy life-styles (APA, 2015) when compared to earlier generations. However, not only cohort effects are to be 
considered, as both developmental and contextual interpretations have been supported (Folkman et al., 1987). Hence, the developmental framework contributes to our understanding of how younger and mature couples differ by examining not only the individual mastery of coping skills through the life-span, but also focusing on couple relations through time. While there is little disagreement that marital satisfaction decreases with time (Bradbury \& Karney, 2014), the literature about close relationships has provided over the last decades evidence for more numerous relational and psychological difficulties for newlyweds and younger families (Amato \& HohmannMarriott, 2007; Bradbury \& Karney, 2014; Carra' Mittini, 1999; Carstensen et al, 2011; Carstensen, Fung, \& Charles, 2003; Chalmers \& Milan, 2005; Luong, Charles \& Fingermann, 2011; Scabini \& Cigoli, 2000; Scabini \& Iafrate, 2003; Scabini \& Rossi, 2012; van Steenbergen, Kluwer, \& Karney, 2011; Woszidlio \& Segrin, 2013). Researchers have recently discovered the existence of different trajectories within the first years of marriage which are associated to marital dissolution. The most significant reduction in marital satisfaction is reported in couples who had low satisfaction at baseline (Lavner, Bradbury, \& Karney, 2012) or greatest expectations about the quality of their relationships (Lavner, Karney \& Bradbury, 2013). Increased rates of divorce after 4 and 10 years of marriage were associated to personality traits, stress, aggression and poor communicative behaviors (Lavner \& Bradbury, 2010). Investigations of the effects of personality traits and stressful events on marital satisfaction in recently married couples confirmed that partners' occupation, work interference, and family stress were negatively associated with marital satisfaction in younger couples (van Steenbergen, Kluwer, \& Karney, 2011; Woszidlio \& Segrin, 2013). Furthermore, economic hardship 
has been associated with higher rate of conflict (Halliday Hardie \& Lucas, 2010), lower life satisfaction, higher pessimism (Haid \& Seiffge-Krenke, 2013) and negative communication (Williamson, Karney, \& Bradbury, 2013) for this group. In constrast, a study by Neff and Broady (2011) highlighted that adaptation to moderately stressful events early in the marriage is associated to reduced stress spillover effect, greater selfefficacy and marital adjustment; suggesting that practicing stress adaptation strategies in the early years of marriage can lead to increased ability to cope with stress at a later stage of the couple's life. It is then possible to affirm that the early years of marriage represent a crucial time for the long-term fate of the relationship and the likelihood for marital dissolution.

When dyadic coping has been investigated among different age groups and cohorts, very interesting results emerged. Initial studies showed that older couples perform consistently better than younger ones (Berg and Upchurch, 2007; Blanchard-Fields \& Coats, 2008; Hoppman, Coats \& Blanchard-Fields, 2008; Revenson 2003; Revenson \& Pranikoff, 2005). More recent data highlighted unique aspects of dyadic coping across younger and older dyads. Iafrate et al. (2012) examined perceived similarity and understanding (i.e., the other partner's dyadic coping responses vs. self-report perception) in young and mature couples. Stereotypical similarity was higher in younger couples, while understanding was higher in mature couple relationships. The authors conclude that for younger partners unique understanding was negatively associated with relationship quality, probably because they experience a higher need of idealization of the relationship. Furthermore, dyadic coping in younger couples seems to be influenced by the example of parental figures and the perception of positive family heritage (Donato et 
al., 2012; Donato, Iafrate, \& Barni, 2013; Iafrate, Donato, \& Bertoni, 2013). However, in a study by Bertoni et al. (2007) younger couples (3 -10 years) engaged in dyadic coping more frequently than couples who have been together for more than 20 years, showing also better performances in stress communication and marital satisfaction; a result clearly in contrast with the literature.

A recent study analyzed the moderating role of dyadic coping on the association between stress and partners' well-being in three age cohorts (Cohort 1: 20-35y.o.; Cohort 2: 40-55y.o.; Cohort 3 65-80 y.o.). Researchers revealed that couple in middle adulthood present the higher values of stress, followed by the younger group. Surprisingly, among older couples both partners reported higher negative support (Vedes, Nussbeck \& Bodenmann, 2015). For younger women their well-being was affected by stress and dyadic coping-especially by negative supportive behaviors- while males' quality of life was mostly influenced by individual coping and contextual factors. Among middle-age couples the well-being of both partners was influenced by stress and dyadic coping. While for women investigators found an actor effect, the well-being of male partners appeared to be more dependent on the dyadic coping of the wife. These differences disappeared in the late-adulthood group. It is therefore possible to conclude that the relationship among stress, dyadic coping and well-being changes across the life course experience especially for men: while in the younger cohort their well-being was influenced by other variables, in middle-age the relationship between the dyadic coping style of the partner and the individual's well-being become more relevant. The authors suggest that probably men between $40-55$ years of age experience difficulties managing 
their roles and integrating traditional and equalitarian gender roles in the couple relationship.

For the purpose of our investigation it becomes clear that we are moving away from considerations associated only to the presentation of positive or negative dyadic coping styles in the dyad in favor of theoretical reflections that address the complexity associated with each stage of the couple experience. In conclusion, the relationship between age, stress and coping processes is highly complex, reflecting a combination of age, cohort and contextual effects that future studies need to acknowledge and account for.

\section{Limitations}

Although recent evidence suggests that this framework is widely used among family researchers (i.e. Hill, Erickson, Fellows, Martinengo \& Allen, 2012; Kamp Dush \& Taylor, 2012; Sassler, 2010; Thomson, Winkler-Dworak \& Kennedy, 2013), two types of limitations interfere with the application of the Life Course Developmental Framework to the study of dyadic coping with cancer. The first one is more theoretical and involves the fact that Family Development Theory did not take into account divorce, death of a spouse, stepfamilies, childless couples, cohabitating or gay and lesbian couples. The theory normalizes just one type of family, disregarding the variety in family constellations and the influence of culture on the couple relationship and its associated coping behavior. On the contrary it is well know that nowadays couples are getting married later in their life, with the consequence that some stages are postponed (Qualls, 1997), while other typologies of couples will never undergo the same developmental process. Similarly, a large percentage of marriages in the USA end in divorce, with the literature estimating a range between 40\% and 50\% (Birditt, Brown, Orbuch, \& 
McIlvane, 2010; Bramlett \& Mosher, 2002; Rogers, 2004). It is possible to theorize that younger couples are already dealing with more difficulties in their relationships even before the diagnosis. Another key issue is the definition of the process of development. Mattessich and Hill's (1987) view of family development was influenced by child development and relied on ontogenetic developmental perspectives. Furthermore, the model used behaviors to infer norms (White, 1991; White et al., 2015). Changes in Family Development Theory are seen as discrete jumps, while more realistically they should be conceptualized as gradual, continuous changes.

The second order of limitations is more applicative. The framework has been criticized for being only descriptive and not heuristic, affected by little predictive power. Early studies failed to include family characteristics like race, socio-economic status, ethnicity, and family structure (Karney, Kreitz, \& Sweeney, 2004). On the contrary, researchers who have examined differences in coping and marital satisfaction among diverse racial groups highlight how Black americans, compare to Whites, begin their marriages with more numerous risk factors and stressors which may spill over to their marital satisfaction (LaVeist, 2005; Orbuch, Veroff, \& Hunter, 1999; Veroff, 1999). For example, black couples reported lower income, were more likely to have children before marriage, and to have cohabited (Orbuch, Veroff, Hassan, \& Horrocks, 2002). These stressors, coupled with the quality of interpersonal relationships, may influence couples' coping mechanism and the long term risk of divorce (Orbuch et al., 2002).

Finally, another consideration pertains the use of the theory in the study of couples coping with cancer. From the review of the literature presented earlier, it clearly emerges that age and length of relationships are the two variables used to identify younger and 
older couples. However they are often confounded with each other and the literature hasn't been able to identify all the factors that contribute to the identification of a "younger couple". As beautifully described by Folkman et al. (1987) "the question is whether these findings are the result of differences in what the two age groups were coping with (the contextual interpretation), developmental stages over the life course, or cohort effects"(ibidem, p.182). Future investigations are needed to clarify which interpretation or which combination of factors contributes to explain the experience of dyadic coping with cancer across the life trajectory.

\section{Summary}

This chapter has provided a review of the foundational theoretical literature of dyadic coping. This review revealed that dyadic coping is essential to understand the experience of couples coping with a diagnosis of cancer, as it promotes adjustment, marital satisfaction, and well-being for both patients and partners. Among the models emerging through the years, systemic perspectives have received more empirical support. Among them, the Relational-Cultural model has been identified as the guiding framework for the present study because of its ability to see the dyadic coping efforts as the result of existing relational competencies, and because of its application in the context of close

relationships and cancer. Hence, the resulting conceptual model of the study examining the relationship among mutuality, dyadic coping and quality of life has been presented. In the second part of the chapter theories of reference (Systems Theory, Transactional Theory of Stress and Coping and Relational Cultural Theory) have been reviewed and critiqued for their ability to contribute to the study of dyadic coping. As the study focuses 
on a comparison of younger and older couples, the Life Course Developmental

Framework has been introduced and discussed to highlight the relevance of individual and couple development in coping. 


\section{CHAPTER III: METHODOLOGY}

The third chapter presents the current study and its methodology. In particular, research goals and hypotheses, research design, data source, sampling, operationalization of variables and data analysis plan are outlined.

\section{Research Goal and Hypotheses}

The purpose of the study was to investigate younger couples' adaptation and coping with the diagnosis of breast cancer, by comparing them to a group of older dyads. More specifically, given the increasing interest in the psychosocial literature about the function dyadic coping has for marital quality and individual's well-being in the context of a chronic illness, the relationship among mutuality, dyadic coping and quality of life of patients and partners has been examined between these two groups to test for empirical evidence a conceptual framework inspired by the Relational-Cultural Model of dyadic coping (Kayser et al., 2007).

The following overarching research questions guided the study:

1. Does a diagnosis of breast cancer affect younger patients and their partners'quality of life, dyadic coping, communication and mutuality more negatively than older couples? 
2. How does dyadic coping affect the quality of life of younger patients and their partners?

3. How does mutuality affect dyadic coping styles of breast cancer patients and their partners?

4. Does dyadic coping mediate the relationship between mutuality and quality of life of patients and their partners?

Based on the questions stated above, the following specific aims and hypotheses were formulated:

Specific Aim 1: To describe differences between younger and older couples on quality of life, dyadic coping, communication and mutuality

Hypothesis 1.1: Younger patients with breast cancer will report lower quality of life, mutuality, communication, and higher negative dyadic coping styles compared to older breast cancer patients

Hypothesis 1.2: Younger partners will report lower quality of life, mutuality, communication, and higher negative dyadic coping styles compared to older partners of breast cancer patients

Hypothesis 1.3: The association of quality of life scores between patients and partners in older couples will be higher than the association of quality of life scores of patients and partners in younger couples

Hypothesis 1.4: The association of dyadic coping scores between patients and partners in older couples will be higher than the association of dyadic coping scores of patients and partners in younger couples

Specific Aim 2: To examine the relationship between dyadic coping and quality of life for younger breast cancer patients and partners

Hypothesis 2.1: Positive forms of dyadic coping (Stress Communication, Common Dyadic Coping, Positive Dyadic Coping), will be associated to higher levels of quality of life for younger breast cancer patients 
Hypothesis 2.2: Positive forms of dyadic coping (Stress Communication, Common Dyadic Coping, Positive Dyadic Coping), will be associated to higher levels of quality of life for partners of younger breast cancer patients

Hypothesis 2.3: The relationship between dyadic coping styles and quality of life for patients and partners will be influenced by socio-demographic, clinical, relational and mental health variables

Specific Aim 3: To assess the actor and partner effects of mutuality on dyadic coping among breast cancer patients and partners and to identify differences by age group

Hypothesis 3.1: Patients' perceived mutuality will influence their own dyadic coping style and their partners' dyadic coping style

Hypothesis 3.2: Partners' perceived mutuality will influence their own dyadic coping style and the patients' dyadic coping style

Hypothesis 3.3: Differences in actor and partner effects of mutuality on dyadic coping exist by age group, between younger and older dyads

Specific Aim 4: To test the mediating role of dyadic coping on the relationship between mutuality and quality of life of patients and partners and to identify differences by age group

Hypothesis 4.1: Self-reported dyadic coping score of patients will mediate the relationship between mutuality and quality of life of breast cancer patients

Hypothesis 4.2: Self-reported dyadic coping score of partners will mediate the relationship between mutuality and quality of life of partners of breast cancer patients

Hypothesis 4.3: Differences exist by age group, between younger and older dyads

\section{Research Design}

The study is a secondary data analysis of the baseline data of a more extensive randomized clinical trial (RCT) aimed at evaluating the effectiveness of a couple-based psychosocial intervention for breast cancer patients and their partners. The design is a cross-sectional survey of socio-demographic, clinical and psychosocial measures. 


\section{Data Source and Sampling}

Participants have been recruited among adult patients newly diagnosed with earlystage non metastatic breast cancer in the hematology/oncology clinics of two medical centers in the northeast of the United States. Inclusion criteria were:

(1) having received a diagnosis of primary non metastatic breast cancer within the last 3 months;

(2) being currently involved in a close relationship with a partner;

(3) being older than 18 years of age;

(4) receiving routine clinical care at the hematology/oncology clinics of the participating sites;

(5) being able to understand English.

Individuals whose diagnosis was rated as Stage 0 were excluded from the study because the participating institutions had different clinical definitions about this condition. Similarly, women with Stage 4 diagnosis of breast cancer were excluded because of the advanced stage of the disease. The study was inclusive of heterosexual and same-sex relationships, however only one same-sex couple was enrolled. Two research coordinators employed at the two participating institutions identified and referred potential participants, according to the IRB-approved protocol. Recruiters met with potential participants at the clinic to present the study or letters were sent to them at their home address, including a study brochure, a decline card, and pre-stamped return envelopes. If interested, participants completed informed consent forms and received a survey questionnaire. Patients and partners completed their questionnaires and returned them in two separate pre-stamped envelopes. Ninety-four patients and ninety partners 
returned their baseline questionnaires to the research team, with data available for 86 dyads.

An agreed definition of who is a "younger woman" with cancer is still elusive and multiple approaches have been identified in the literature, with authors classifying as "younger" women below 40 years of age ${ }^{15}, 45^{16}, 50^{17}$ or even 60 years of age ${ }^{18}$. The literature about breast cancer usually refers to "younger women" as those in their reproductive years (Hulvat \& Jeruss, 2009). According to the National Institute on Aging (2015) the mean age of menopause is at 51 for American women with a peri-menopause stage between 47 and 51 . Hence, in the present study younger couples were identified as those where the patient was $\leq 45$ y.o. at time of diagnosis. The decision to use this age as cut-off to identify the two groups in the present study is also supported by the fact that, when survivorship has been investigated in younger women with breast cancer, researchers have usually enrolled women from the age of 50, suggesting that 45 years of age would have been the time of diagnosis (Champion et al., 2014). A total of 35 dyads met this indication, and they were compared with the remaining 51 couples considered as “older couples".

\footnotetext{
${ }^{15}$ See: Anderson et al., 2011; Kruger et al., 2009; Pinto, 2013; Rosenberg et al., 2013; Salsman et al., 2014; Sanford et al., 2014; Thewes et al., 2005; Wenzel et al., 1999.

${ }^{16}$ See: Antoine et al., 2012; Bifulco et al., 2012; Champion et al., 2014; Christophe et al., 2015b; Stava, Lopez \& Vassilopoulou-Sellin, 2006; Vanlemmens et al., 2012a; Vanlemmens et al., 2012b.

${ }^{17}$ See: Bantema-Joppe et al., 2015; Bloom, Stewart, Oakley-Girvan, Banks \& Shema, 2012; Burwell et al., 2006; Herbenick et al., 2008; Manuel et al., 2007; Ventura et al., 2013.

${ }^{18}$ See: Phillips et al., 2008; So et al., 2011.
} 


\section{Operationalization of Variables}

Socio-Demographic Variables were measured with individual items of age, race, number of children, education, income, occupation, and religious affiliation.

Relationship Characteristics were measured by items assessing length of relationship, marital status, and cohabitation.

Quality of Life - Patients. Quality of Life has been defined as the "individuals" perception of their position in life in the context of culture and value systems in which they live and in relation to their goals, expectations, standards and concerns. It is a broadranging concept affected in a complex way by the person's physical health, psychological state, level of independence, social relationships personal beliefs and their relationship to salient features of their environment"(WHO, 1993). When working within the context of health, quality of life is often referred to as health-related quality of life (HRQOL), which indicates the impact a medical condition or its treatment has on the expected physical, emotional, and social well-being (Salsman, Pearman \& Cella, 2013; Cella, 1994; Cella, 1995).

The quality of life of women diagnosed with breast cancer was measured by the Functional Assessment of Cancer Therapy-Breast (FACT-B) Scale (Brady et al., 1997; Cella et al., 1993). The FACT-B (Version 4) is a 37-item measure that contains four general subscales assessing the physical, social/family, emotional, and functional wellbeing of the individual, along with the breast cancer-specific subscale that assesses concerns of particular relevance to breast cancer patients (e.g., body image, arm swelling and tenderness). Patients were invited to indicate how true each statement has been for 
them in the previous seven days, and items are rated on a 5 point Likert scale ranging from "Not at All" (0) to "Very Much" (4).

The FACT-B consists of five subscale scores: physical well-being (PWB), social/family well-being (SWB), emotional well-being (EWB), functional well-being (FWB) and additional concerns (BCS), with higher scores indicating higher quality of life. From these subscale scores, two assessment total scores were calculated: the FACTB total score, and the FACT-G score. The FACT-B total score is calculated by summing all five un-weighted subscale scores, with total scores in the range of 0-136. The FACTG score is calculated by summing PWB, SWB, EWB, and FWB scores (i.e., excluding the Breast Cancer Specific subscale), with scores in the range of 0-108. Administration and scoring guidelines are available on the website http://www.facit.org/FACITOrg, and subscales and total scores were calculated using the SPSS syntax provided by the authors of the instrument. Recently, general population and cancer patients' normative data for the FACT-G were released (Brucker, Yost, Cashy, Webster, \& Cella, 2005).

The FACT-B has been extensively used in psychosocial oncology research and has demonstrated to have high validity and internal consistency (Brady et al., 1997; Cella et al., 1993; Overcash, Extermann, Parr, Perry, \& Balducci, 2001; Webster, Cella \& Yost, 2003; Webster, Odom, Peterman, Lent, \& Cella, 1999; Winstead-Fry \& Schultz, 1997). In its validation study, Cronbach's alpha for the total score was .90 , with subscale alpha coefficients ranging from .63 to .86 (Brady et al., 1997). Evidence supported test-retest reliability, as well as convergent and divergent validity (Brady et al., 1997; Cella et al., 1993; Overcash et al., 2001; Webster et al., 2003; Webster et al., 1999; Winstead-Fry \& Schultz, 1997). For the purpose of the present investigation, the five subscales and the 
FACT-G and FACT-B total scores were used. Similarly to the data available in the literature, very high internal consistency has been registered in this sample (FACT-G $\alpha=.90$, FACT-B total score $\alpha=.90, \operatorname{PWB} \alpha=.88, \operatorname{SWB}=.81, \mathrm{EWB} \alpha=.83$, FWB $\alpha=.85$, $\operatorname{BCS} \alpha=.81)$.

Quality of Life - Partners. The quality of life of partners was measured with two instruments: the Emotional Functioning subscale from the Quality of Life Questionnaire for Spouses (QL-SP) (Ebbesen, Guyatt, McCartney, \& Oldridge, 1990) and the Illness Intrusiveness Ratings Scale (IIRS) (Binik, Chowanec, \& Devins, 1990).

The Quality of Life Questionnaire for Spouses (QL-SP) consists of a total of 26 items measuring the emotional, physical and social function of spouses of a patient with chronic illness. In particular, the Emotional Function Dimension (14 items) examines the well-being of the individual in the previous two weeks by rating on a 7 point Likert scale anxiety, depression, concerns, frustration and helplessness (Ebbesen et al., 1990). Total scores on the scale range from 7 to 98 , with higher scores indicating better level of functioning. Construct validity was rated as good and the instrument has correlated highly with anxiety and depression based on other valid scales (Feldman \& Broussard, 2006). The scale demonstrated high internal consistency in previous studies (Feldman \& Broussard, 2006; Iafrate et al., 2012), and this evidence was supported by a Cronbach's alpha value of .91 in the present sample.

The Illness Intrusiveness Ratings Scale measures the amount of disruption or interference of the partner's illness and treatment on 13 dimensions of the respondent's life (Devins. 1994; Devins et al., 1983). These dimensions include health, work, recreation, financial situation, sex life, and other social relations. Each domain is rated on 
a 7 point Likert Scale, from "Not very much" (1) to "Very Much" (7). When respondents consider an item not to be applicable, a score of one is entered, indicating that the illness and/or its treatment do not interfere very much with this life domain. The total score ranges from 13 to 91, with higher scores indicating greater impact of the patient's illness on the partner. Several studies support the reliability and validity of the instrument (Binik et al., 1990; Devins, 1994; Devins et al., 1990). Previous findings indicate that there is a significant association between perceived intrusiveness and negative mood, self-efficacy, marital adjustment and life satisfaction (Devins, Hunsley, Mandin, Taub, \& Paul, 1997; Devins, 1994). Internal consistency of the scale is high: a recent systematic review conducted on the application of the IIRS on 36 chronic disease groups highlighted that Cronbach's alpha scores ranged from the .80 's to the .90 's (Devins, 2010). In the present sample the Cronbach's alpha was .88 .

Dyadic Coping. Dyadic coping was measured by the Dyadic Coping Scale (Bodenmann, 2000). This self-report questionnaire assesses stress communication and dyadic coping as perceived by each partner about his or her own coping ("what I do when I am stressed" and "what I do when my partner is stressed"), each partner's perception of the other's coping ("what my partner does when I am stressed"), and each partner's view of how they cope as a couple ("what we do when we are stressed as a couple").

In this version each item (for a total of 61) is measured on a 6-point Likert scale, with responses ranging from "Very rarely" (1) to "Always" (6). The Dyadic Coping Scale contains five subscales: Stress Communication, Common, Positive, Hostile and Avoidance of Dyadic Coping. Plus, a total dyadic coping score can be calculated as the sum of the single items included in the subscales. 
1. Stress Communication was measured as the mean of 5 items which assess the partners' ability to communicate emotion- and problem-focused stress, with higher scores indicating better communication. Examples are "I ask my partner to do things for me when I have too much to do", "I try to hide my stress from my partner so that he/she does not notice it", and "I tell my partner openly how I feel and that I would appreciate his/her support".

2. Common Dyadic Coping was examined by 13 items which measure both partners' ability to participate in the coping process in a coordinated and symmetrical manner. Examples of problem focused Common Dyadic Coping include joint problem solving, equal division of tasks and joint information seeking, while among emotion-focused strategies the questionnaire list activities that promote the perception of unity as a couple, like spiritual activities and engagement in joint relaxation exercises. Examples of items of this subscale are "We are supportive of each other and help one another out", "We help one another to put the problem in perspective and see it in a new light", and "We caress one another and make love".

3. Positive Dyadic Coping refers to the use of supportive dyadic coping strategies like the provision of practical help, giving relevant information, advice, understanding and helping to relieve tension. Traditionally, positive coping strategies are used in an attempt to assist the member of the dyad who is perceived "generally or presently less equipped with the coping resources required to achieve the maintenance or restoration of an adaptive state" (Bodenmann, 1997, p. 138). Examples of items included in this subscale are: 
"My partner gives me feeling that he/she understands me"; "My partner listens to me and gives me the opportunity to communicate the entire situation", and "My partner takes on things that I normally do in order to help me out".

4. Hostile Dyadic Coping occurs when the stress signals of one partner originate a hostile behavior by the other. Responses or behaviors that can be considered hostile include distancing, ridicule, sarcasm, clear disinterest and minimizing the emotional experience of stress of the other. Scale items that are included in this subscale are: "I make fun of my partner's stress", "I let my partner know that I do not want to be bothered with his/her problems", and "Although my partner makes time for me, his/her thoughts are somewhere else".

5. Avoidance of Dyadic Coping describes ambivalent and superficial coping responses, where authentic engagement is absent (Bodenmann, 1997;

Bodenmann, 2005). In the present scale three items measure avoidance of dyadic coping, with higher scores indicating that these behaviors are used as a response to the stress perceived by the partner. Examples of such items are "When my partner is stressed, I tend to get out of his/her way", and "When my partner is stressed I tend to withdraw".

Satisfactory psychometrics of the questionnaire have been reported. A previous work using the same version of the Dyadic Coping Scale with a sample of male partners reported acceptable alphas for each subscale: stress communication $(\alpha=.63)$, common dyadic coping $(\alpha=.84)$, positive dyadic coping ( $\alpha=.95)$, hostile dyadic coping $(\alpha=.76)$, and avoidance of dyadic coping ( $\alpha=.73$ ) (Feldman \& Broussard, 2006). In this study, reliability scores ranged from .68 to .96 for patients, and from .68 to .95 for partners. 
Mutuality. Mutuality refers to a "bidirectional movement of feelings, thoughts, and activity between persons in relationship" (Genero, Baker-Miller, Surrey, \& Baldwin 1992; p.1). From a relational perspective (Jordan, 1986), mutuality is described as a series of interactions that contribute to the well-being of the relationships (Genero et al., 1992). In the present study it is measured by the Mutual Psychological Development Questionnaire (MPDQ) (Genero et al., 1992). The instrument consists of 22 items assessing six conceptual dimensions of mutuality: empathy, engagement, authenticity, zest, diversity and empowerment on a 6 point scale ranging from "Never"(1) to "All the time" (6). The scale is organized in two subscales: one is about how the respondent perceives his or her own empathic responsiveness and the other subscale is the respondent's perception of the partner's empathic responsiveness. Hence, both subscales need to be totaled together in order to compute the level of mutuality reported by the individual about the close relationship investigated.

Results of an initial validation study (Genero et al., 1992) conducted on more than 300 subjects recruited through introductory psychology courses, continuing education programs and community health care centers indicated high inter-item reliability (alpha coefficients ranged from .89 to .94$)$. Construct and concurrent validity were demonstrated with a high correlation between the MPDQ and measures of social support, relationship satisfaction and relationship cohesion. There was a large negative correlation between the MPDQ and depression as measured by the Center for Epidemiological Studies Depression Scale (CES-D). Test-retest reliability of the scale was satisfactory, and high inter-item consistency was replicated (Genero et al., 1992). The scale has been used extensively in the literature about close relationships and its psychometrics confirmed 
(i.e.: Coan, Kasle, Jackson, Schaefer, \& Davidson, 2013; Kruger, 2005; Liang et al., 2002), with Cronbach's alpha between .89 and .94 . In the present sample reliability scores were high for both patients $(\alpha=.93)$ and partners $(\alpha=.91)$.

Communication. The Couple Communication Scale by Barnes and Olson (1986) was administered to participants to assess their communication. The scale, which is composed by 10 items ranging from "Definitely False" (1) to "Definitely True" (7), examines individual's feelings, beliefs, and attitudes about the communication in the relationship with the partner. Items focus on the level of comfort felt by both partners in being able to share important emotions and beliefs with each other, the perception of a partner's way of giving and receiving information, and the respondent's perception of the quality of the communication between the two members of the dyad. As part of the ENRICH Marital Inventory (Olson, Fournier, \& Druckman, 1986), the scale has been extensively utilized in psychosocial research and its psychometrics confirmed in numerous research studies. The total communication score, ranging from 10 to 50 , has been used in the study. In the present sample Cronbach's alpha was .79 for patients and .86 for partners, while in the original validation study a reliability score of .68 was registered.

Clinical Variables. Patients were asked to indicate if they were currently taking medications when the questionnaire was administered. In particular, a variable indicates if the patient has received chemotherapy or not as part of her cancer treatment.

Mental Health History and Treatment for Depression. Mental health of patients and partners was assessed through questions about history and treatment for mental health diagnosis in the family and for the individual participant. Breast cancer patients were also asked if they have received treatment before or after the diagnosis. 
Table 3. Operationalization of Variables for Patients and Partners

\begin{tabular}{|c|c|c|}
\hline $\begin{array}{l}\text { Operationalization of } \\
\text { Variables }\end{array}$ & Patient & Partner \\
\hline Socio-Demographic & Age & Age \\
\hline \multirow[t]{6}{*}{ Variables } & Race & Race \\
\hline & Number of Children & Number of Children \\
\hline & Education & Education \\
\hline & Income & Income \\
\hline & Occupation & Occupation \\
\hline & Religious Affiliation & Religious Affiliation \\
\hline Clinical & Medications (Y/N) & \\
\hline Variables & Chemotherapy (Y/N) & \\
\hline Relational & Length of relationship & Length of relationship \\
\hline \multirow{2}{*}{ Characteristics } & Marital status & Marital status \\
\hline & Cohabitation & Cohabitation \\
\hline \multirow[t]{2}{*}{ Quality of Life } & $\begin{array}{l}\text { Functional Assessment of } \\
\text { Cancer Therapy-Breast } \\
\text { (FACT-B) Scale }\end{array}$ & $\begin{array}{l}\text { Quality of Life Questionnaire } \\
\text { for Spouses (QL-SP) }\end{array}$ \\
\hline & & $\begin{array}{l}\text { Illness Intrusiveness Ratings } \\
\text { Scale (IIRS) }\end{array}$ \\
\hline \multirow[t]{2}{*}{ Mutuality } & Mutual Psychological & Mutual Psychological \\
\hline & $\begin{array}{l}\text { Development Questionnaire } \\
\text { (MPDQ) }\end{array}$ & $\begin{array}{l}\text { Development Questionnaire } \\
\text { (MPDQ) }\end{array}$ \\
\hline Dyadic Coping & Dyadic Coping Scale & Dyadic Coping Scale \\
\hline Communication & Couple Communication Scale & Couple Communication Scale \\
\hline Mental Health & History of Mental Health & History of Mental Health \\
\hline \multirow{3}{*}{ History } & Issues in the Family & Issues in the Family \\
\hline & Family Member with Mental & Family Member with Mental \\
\hline & Health Diagnosis & Health Diagnosis \\
\hline \multirow{3}{*}{$\begin{array}{l}\text { Treatment for } \\
\text { Depression }\end{array}$} & Previous Diagnosis of & Previous Diagnosis of \\
\hline & Depression $(\mathrm{Y} / \mathrm{N})$ & Depression $(\mathrm{Y} / \mathrm{N})$ \\
\hline & $\begin{array}{l}\text { Timing of Diagnosis } \\
\text { (Before/After Diagnosis) }\end{array}$ & \\
\hline
\end{tabular}


Table 4. Score calculation and Reliability of Instruments

\begin{tabular}{|c|c|c|c|}
\hline Instrument & Score calculation & $\begin{array}{l}\text { Cronbach's } \alpha \\
\text { Patients }\end{array}$ & $\begin{array}{l}\text { Cronbach's } \alpha \\
\text { Partners }\end{array}$ \\
\hline \multicolumn{4}{|l|}{ Quality of Life - } \\
\hline $\begin{array}{l}\text { Physical Well-being } \\
\text { (PWB) }\end{array}$ & Sum items $1-7$ & .88 & - \\
\hline $\begin{array}{l}\text { Social/Family Well- } \\
\text { being (SWB), }\end{array}$ & Sum items 8-14 & .81 & - \\
\hline $\begin{array}{l}\text { Emotional Well-being } \\
\text { (EWB), }\end{array}$ & Sum items $15-20$ & .83 & - \\
\hline $\begin{array}{l}\text { Functional Well-being } \\
\text { (FWB) }\end{array}$ & Sum items 21-27 & .85 & - \\
\hline $\begin{array}{l}\text { Additional Concerns } \\
\text { (BCS). }\end{array}$ & $\begin{array}{l}\text { Sum items } 28-36 \text { (Item } 37 \text { not } \\
\text { currently scored) }\end{array}$ & .81 & - \\
\hline FACT-B & $\begin{array}{l}\text { Sum of PWB, SWB, EWB, FWB, } \\
\text { and BCS }\end{array}$ & .90 & - \\
\hline FACT-G & $\begin{array}{l}\text { Sum of PWB, SWB, EWB, and } \\
\text { FWB }\end{array}$ & .91 & - \\
\hline \multicolumn{4}{|l|}{ Quality of Life - } \\
\hline $\begin{array}{l}\text { Quality of Life } \\
\text { Questionnaire for } \\
\text { Spouses (QL-SP) }\end{array}$ & Sum items 1-14 & - & .91 \\
\hline $\begin{array}{l}\text { Illness Intrusiveness } \\
\text { Scale (IIRS) }\end{array}$ & Sum Items 1-13 & - & .88 \\
\hline \multicolumn{4}{|l|}{ Dyadic Coping } \\
\hline Stress Communication & Mean Item 1, 2, 3, 4 and 5Reversed & .68 & .68 \\
\hline $\begin{array}{l}\text { Common Dyadic } \\
\text { Coping }\end{array}$ & $\begin{array}{l}\text { Mean Item } 49,50,51,52,53,54,55 \text {, } \\
56,57,58,59,60,61\end{array}$ & .86 & .83 \\
\hline Positive Dyadic Coping & $\begin{array}{l}\text { Mean Item } 6,7,9,10,12,13,15,17 \\
18,21,22,26,27,29,30,32,34,36 \\
39,43,44,49,50,52,53,54,55,56 \\
57,59\end{array}$ & .96 & .95 \\
\hline Hostile Dyadic Coping & $\begin{array}{l}\text { Mean Item 8, 11, 14, 16, 28, 31, 35, } \\
37,42\end{array}$ & .80 & .70 \\
\hline $\begin{array}{l}\text { Avoidance of Dyadic } \\
\text { Coping }\end{array}$ & Mean Item 33, 46, 58 & .68 & .68 \\
\hline Total Dyadic Coping & $\begin{array}{l}\text { Sum Items } 1,2,3,4,5,6,7,9,10, \\
11,12,13,14,15,16,17,18,21,22, \\
26,27,28,29,30,31,32,33,34,35, \\
36,37,39,42,43,44,46,49,50,52, \\
53,54,55,56,57,58,59\end{array}$ & .91 & .90 \\
\hline Mutuality & & & \\
\hline $\begin{array}{l}\text { Mutual Psychological } \\
\text { Development } \\
\text { Questionnaire (MPDQ) }\end{array}$ & $\begin{array}{l}\text { Mean of Items } 1-22 \text {, with items } 2,4 \text {, } \\
6,8,10,12,14,16,18,20,22 \\
\text { reversed }\end{array}$ & .93 & .91 \\
\hline
\end{tabular}


Communication

Couple

Sum of Items 1-10, with items $2,3, \quad .79$

.86

Communication Scale

$4,5,6,7,9$ reversed

\section{Statistical Analysis}

After obtaining Institutional Review Board approval to review and access these data, IBM SPSS Statistics 22 was used for data management, data screening and preliminary data analyses. Descriptive statistics were obtained for all the variables compiling frequency tables, histograms and bar graphs. Missing data and outliers were examined by conducting a Missing Value Analysis (MVA) and univariate outliers were examined through the SPSS Explore command and the use of Box Plots. Among the numerous imputation procedures currently available, mean substitution was implemented to handle missing data on the key variables. Skewness and Kurtosis were also investigated to test for normality through SPSS Frequencies and Descriptive analysis (Meyers, Gamst \& Guarino, 2006). Kolmorogov-Smirnov test was employed to detect departures from normality. When non-normal distribution was detected, variables have been transformed according to Tabachnick and Fidell (2007).

Pearson $r$ correlations and bivariate scatterplots were used to assess the linear relationship observed between socio-demographic, clinical and psychosocial measures, with specific attention for the variables of interest for the study. Comparisons of demographic characteristics between patients and spousal caregivers were conducted using $t$-tests for continuous variables and chi-square for ordinal and categorical variables.

\section{Specific Aim 1}

Differences between younger and older breast cancer patients on quality of life, dyadic coping, communication, and mutuality have been assessed by calculating $t$-tests 
for independent samples, with a $p$-value smaller of 0.05 considered statistically significant. Similarly, $t$-tests for independent samples were calculated to examine differences between younger and older partners on the same measures. $T$ tests were preferred to ANOVAs because the independent variable (younger vs. older participants) had only two levels (Meyers et al., 2006). To test the hypothesis of higher association of quality of life and dyadic coping existing among older couples, Pearson $r$ productmoment correlations coefficients were used to examine the existence of a linear relationship between these continuous variables.

\section{Specific Aim 2}

The second aim of the study was to investigate the relationship between dyadic coping and quality of life among younger couples. It was hypothesized that positive forms of dyadic coping (Stress Communication, Common Dyadic Coping and Positive Dyadic Coping) were associated with higher quality of life for both younger patients and partners. Pearson $r$ correlations were calculated to assess the relationship between quality of life and dyadic coping styles of younger breast cancer patients and partners' scores. To investigate factors affecting the relationship between dyadic coping and quality of life, Pearson's $r$ product-moment correlations coefficients were calculated between these variables and socio-demographic, clinical, relational and mental health variables of patients and partners. Based on the significant correlations identified with these analyses, separate multiple regressions were calculated to predict quality of life of patients and partners. 


\section{Specific Aim 3}

After conducting descriptive and independent analyses on patients and partners using the conventional individual data file, further analyses were conducted using a dyadic data analysis approach because of the non-independence existing between patients and partners' scores (Kashy \& Snyder, 1995; Kenny, 1996). This association between partners' scores in marital dyads is due to compositional effects (similarities between individuals), partner effects (characteristics of one partner that affect characteristics of the other) and common fate (partners are exposed to similar contextual forces) (Kenny, 1996; Kenny, Kashy \& Cook, 2006). Ignoring non-independence in close relationships has conceptual and methodological ramification (Kashy \& Donnellan, 2012): if the assumption of independence is violated, test statistics and degrees of freedom will be inaccurate and statistical significance will be biased (too liberal: type I error; too conservative: type II error) (Kenny, 1996; Kenny \& Judd, 1986; Kenny, Kashy \& Bolger, 1998; Kenny et al., 2006).

Other two factors to be considered are the nature of the variables under investigation and distinguishability (Kenny et al., 2006). When using dyadic data analysis, variables can be within-dyads, between-dyads or mixed depending on the design of the study and the hypotheses developed by the investigator. Between-dyads variables differ among the single dyads in the sample, but not within the two members of the dyads; therefore they present the same score on the variable. In this case, the age group variable is a between-dyads variable as younger and older dyads were identified based on the age of the patient at the moment of diagnosis. Similarly, length of relationship is a between-dyads variable. Within-dyads variables refer to individual characteristics that 
allow the two members of the unit to be distinguished. The scores of the two partners differ on these types of variables, yet the mean is the same. In the present sample, role represents a within-dyad variable as in each couple involved in the study a patient and a caregiver exist. The final type of variable is a mixed variable, where variations are registered within and between the dyads. For this analysis, mutuality and dyadic coping are mixed variables as the two partners' scores on these may differ between partners and also some couples may present higher or lower average scores (Kashy \& Donnellan, 2012; Kenny et al., 2006). As mentioned earlier, members of the dyad are considered to be conceptually distinguishable if a meaningful variable can be used to differentiate among them. In the case of the present study, dyads were distinguishable because of the role each partner has in relation to cancer (Kashy \& Donnellan, 2012; Kenny et al., 2006).

The file was then restructured into a dyad and pairwise data file, which can be described as a double-entry structure where each record includes the scores of the person and the partner on each variable, with dyad level variables entered only once per dyad. In preparing the dataset for analysis, the data were organized and sorted into individual and dyadic level variables, with dyadic variables comprising of the classification into younger or older couples. Before conducting the analysis, power estimates were obtained using G*Power 3.1.9.2 (Faul, Erdfelder, Buchner \& Lang, 2009; Faul, Erdfelder, Lang, \& Buchner, 2007). The Actor-Partner Interdependence Model (APIM) was used to examine whether individual characteristics, like the mutuality score, have interpersonal consequences on the dyadic coping style reported by each member of the dyad (Kenny et al., 2006; Wittenborn et al., 2013). To facilitate a clear understanding of the analysis, 
partners will be addressed with the term "caregivers" in the presentation of results. The actor effect occurs when the person's score on a predictor variable affects her/his outcome measure, while a partner effect takes place when the partner's score on the predictor variable influences the person's outcome (Kenny, 2013). As depicted in Figure 4, when members of the couple are distinguishable, two actor and two partner effects can be identified. The effect of the patient's and caregiver's mutuality on their own dyadic coping is the actor effect, while the partner effect for patients refers to the effect of caregiver's mutuality on the patient's dyadic coping. Similarly, the partner effect for caregivers refers to the effect of patients' mutuality on the caregiver's dyadic coping (Kenny, 1996; Kenny et al., 2006). Two correlations are present in an APIM model. The first one exists as the two predictors may be already correlated for compositional effects of the dyad. The other correlation is the residual non-independence in the outcome scores, which is left unexplained by the model (Kenny et al., 2006). Using APIM regression, the patient and caregiver predictor variables are regressed on the patient and caregiver outcome variables in a single regression model. In order to conduct the analysis, predictor variables are mean centered and the distinguishing variable is effect coded, with patients coded as 1 and caregivers coded as -1 in this sample. All the analyses were conducted using SPSS Statistics 22. An Actor-Partner Interdependence Model Analysis includes six passages when using multilevel modeling:

- First, an Intra Class Correlation Coefficient (ICC) is calculated for the outcome variables of patients and caregivers. 
- Then, an Onmibus Test of Distinguishability is conducted by comparing a Full Model and a Simplified Model with Maximum Likelihood estimation to evaluate whether treating the dyad as distinguishable improves the fit of the model.

- The third step is the APIM analysis to test whether there are significant role differences, followed by a simple slopes analysis for patients and caregivers in case of role by actor/partner effect interactions. Hence, an interaction model using REML estimation is tested first, followed by a two intercept approach if significant correlations are detected.

- Finally, pseudo $\mathrm{R}^{2}$ are calculated and tested, to indicate the approximate proportion of variance for each dyadic coping style explained by actor and partner mutuality for patients and caregivers. The Deviance Test compares the model with actor and partner effects to the null or empty model.

Results are reported in tables, with a first table that presents multilevel regression coefficients estimating actor and partner effects, and a second one where the simple slope regression coefficients estimating separate mutuality actor and partner effects for patients and caregivers on the different dyadic coping styles are included when a significant interaction role by actor or partner effect exists. Finally, the APIM model is traditionally summarized in the form of a figure where the actor and partner effects are visualized by the use of intersecting arrows. For the present analysis, standardized coefficients are presented for both actor and partner effects. If no significant interaction was registered between role and actor or partner effect, but an average significant actor or partner effect was initially detected in the interaction model, this standardized coefficient was reported in the figure to remind the reader of its significance. Two analyses were conducted: first a 
standard APIM model was calculated with actor and partner mutuality used as predictors of dyadic coping per patients and caregivers. Second, to test if differences exist by age group, separate APIM models were conducted on younger and older dyads ${ }^{19}$.

\section{Specific Aim 4}

Finally, the present study tested the mediating role of dyadic coping on the relationship between mutuality and quality of life of younger patients and partners. Ledermann, Macho, and Kenny (2011) have proposed an extension of the APIM model (Kenny et al., 2006) that can be used to estimate and test mediational processes in dyadic data analysis named APIMeM (Ledermann, Macho, \& Kenny, 2011). However, because the outcome measures of quality of life are different for patients and caregivers, it was not possible to apply a dyadic data analysis approach to the last aim. Instead, a simple mediation model was implemented to test the hypotheses for breast cancer patients and partners separately.

Among the several forms of inter-relationship between variables, mediation implies that an independent variable $\mathrm{X}$ may influence a dependent variable $\mathrm{Y}$ through one or more intervening variables (M) (Hayes, 2013; MacKinnon, 2008; Preacher \& Hayes, 2008). The model includes two sets of antecedent (X and M) and consequent variables (M and Y): X causally influences $\mathrm{Y}$ and $\mathrm{M}$, and M causally influences $\mathrm{Y}$ (Hayes, 2013). The influence of $\mathrm{X}$ on $\mathrm{Y}$ can occur through a direct and an indirect effect. The first one describes the influence of $\mathrm{X}$ on $\mathrm{Y}$ while the second represents how $\mathrm{Y}$ is influenced by $\mathrm{X}$ through a causal sequence in which $\mathrm{X}$ influences $\mathrm{M}$, which then influences $\mathrm{Y}$.

\footnotetext{
${ }^{19}$ Before selecting this strategy an APIM model with the age group effect coded variable was conducted, but the absence of role by age_group interaction prevented from detecting differences in the actor and partner effects for patients and partners in the two samples of dyads.
} 
In the present study, despite data come from a non-experimental design, the logical ordering of the variables of interest was established by the theoretical model of dyadic coping developed by Kayser et al. (2007). According to this model, the effect of a person's mutuality $(\mathrm{X})$ on his or her quality of life $(\mathrm{Y})$, can be the mediated by his/her own dyadic coping style (M). In other words, the effect of the patient's mutuality on her own well-being may be mediated by the self-reported score on dyadic coping. Likewise the effects of the caregiver's mutuality on quality of life may be mediated by his/ her dyadic coping (Figure 5).

Historically, mediation analysis has been conducted using the causal steps approach developed by Baron and Kenny (1986). However, this strategy has received severe critiques in the last few years ${ }^{20}$. Hence, the present mediation analysis has been completed using OLS regression procedures through PROCESS, a computational tool for path analysis-based moderation and mediation developed by Hayes (2013). The tool has been selected also for the possibility to conduct statistical inference of the indirect effect. As the Normal Theory Approach (Sobel Test) has been identified as inaccurate (MacKinnon, Lockwood, \& Williams, 2004), the use of bootstrap confidence intervals has emerged as a more solid option (Hayes, 2009; Hayes, 2013; MacKinnon, 2008; Shrout \& Bolger, 2002; Zhao, Lynch \& Chen, 2010). Initially, the analysis included also age, but the lack of significant results suggested to remove this variable from the model.

\footnotetext{
${ }^{20}$ First, in the Baron and Kenny (1986) approach the existence of an indirect effect is inferred logically and therefore it's more sensible to Type I errors. Second, the identification of $\mathrm{M}$ as a mediator is contingent to the successful rejection of three null hypothesis which significantly affects power. Third, the procedure begins with testing whether $\mathrm{X}$ affects $\mathrm{Y}$ by conducting a hypothesis test for the total effect of $c$, while there is a growing consensus among methodologists that a total effect of $\mathrm{X}$ on $\mathrm{Y}$ should not be a prerequisite to examining the indirect effects (Hayes, 2009; MacKinnon, 2008; Shrout \& Bolger, 2002; Zhao et al., 2010). Finally, since the causal step approach is not based on quantification of the indirect effect, it encourages researchers to think about mediation in qualitative terms.
} 
The simple mediation model was then tested among patients and partner. Finally, given the overall goal of the study, the analysis was conducted on younger and older patients and partners separately.

Table 5. Statistical Analysis Overview

\begin{tabular}{l}
\hline Aim \\
\hline 1. To describe differences \\
between younger and older \\
couples on quality of life, \\
dyadic coping, \\
communication and \\
mutuality
\end{tabular}

\section{Hypothesis}

\section{Hp 1.1}

Younger patients with breast cancer will report lower quality of life, mutuality, communication, and higher negative dyadic coping styles compared to older breast cancer patients

\section{Hp 1.2}

Younger partners will report lower quality of life, mutuality, communication and higher negative dyadic coping compared to older partners of breast cancer patients

\section{Hp 1.3}

The association of quality of life scores between patients and partners in older couples will be higher than the association of quality of life scores of patients and partners in younger couples

\section{Hp 1.4}

The association of dyadic coping scores between patients and partners in older couples will be higher than the association of dyadic coping scores of patients and partners in younger couples

\section{Hp 2.1}

2. To examine the relationship between dyadic coping and quality of life among younger couples
Positive forms of dyadic coping (stress Pearson's $r$ communication, common dyadic coping, correlations positive dyadic coping), will be associated to higher levels of quality of life for younger breast cancer patients.

\section{Hp 2.2}

Positive forms of dyadic coping (stress Pearson's $r$ communication, common dyadic coping, correlations 
positive dyadic coping), will be associated to higher levels of quality of life for partners of younger breast cancer patients

\section{Hp 2.3}

The relationship between dyadic coping styles and quality of life for patients and partners is influenced by sociodemographic, clinical, relational and mental health variables

\section{To assess the actor- partner effects of mutuality on dyadic coping among breast cancer patients and partners and to identify differences by age group}

\section{Hp 3.1}

Patients' perceived mutuality will influence their own dyadic coping style and their partners' dyadic coping style

\section{Hp 3.2}

Partners' perceived mutuality will influence their own dyadic coping style and the patients' dyadic coping style

\section{Hp 3.3}

Differences in actor and partner effects of mutuality on dyadic coping exist by age group, between younger and older dyads

\section{To test the mediating role of dyadic coping on the relationship between mutuality and quality of life of patients and partners and to identify differences by age groups}

\section{Hp 4.1}

Self-reported dyadic coping score of patients and partners will mediate the relationship between mutuality and quality of life of younger breast cancer patients

\section{Hp 4.2}

Self-reported dyadic coping score will mediate the relationship between mutuality and quality of life of partners of younger breast cancer patients

\section{Hp 4.3}

Differences exist by age group, between younger and older dyads
Pearson's $r$ correlations and multiple regression analysis

APIM model for distinguishable dyads
Simple mediation model 
Figure 4. Proposed APIM Model of Mutuality and Dyadic Coping

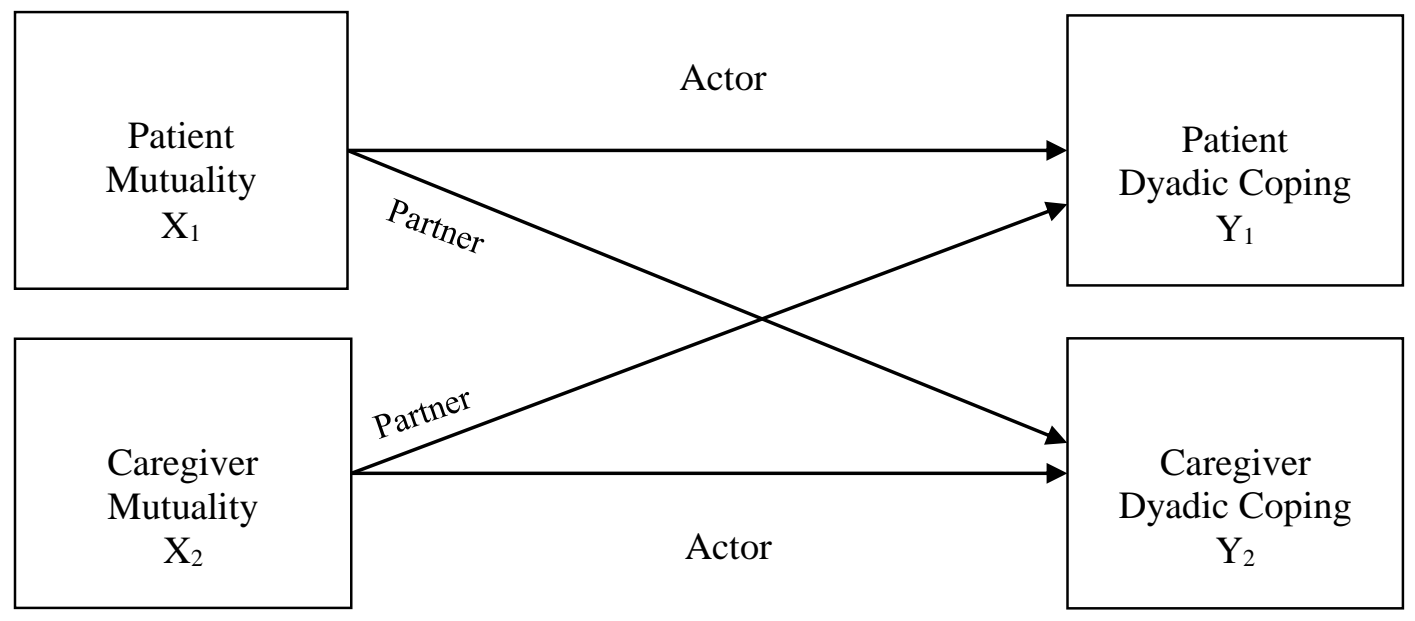

Figure 5. Conceptual diagram of the proposed Simple Mediation Model with Dyadic Coping mediating the relationship between Mutuality and Quality of Life of Patients and Partners
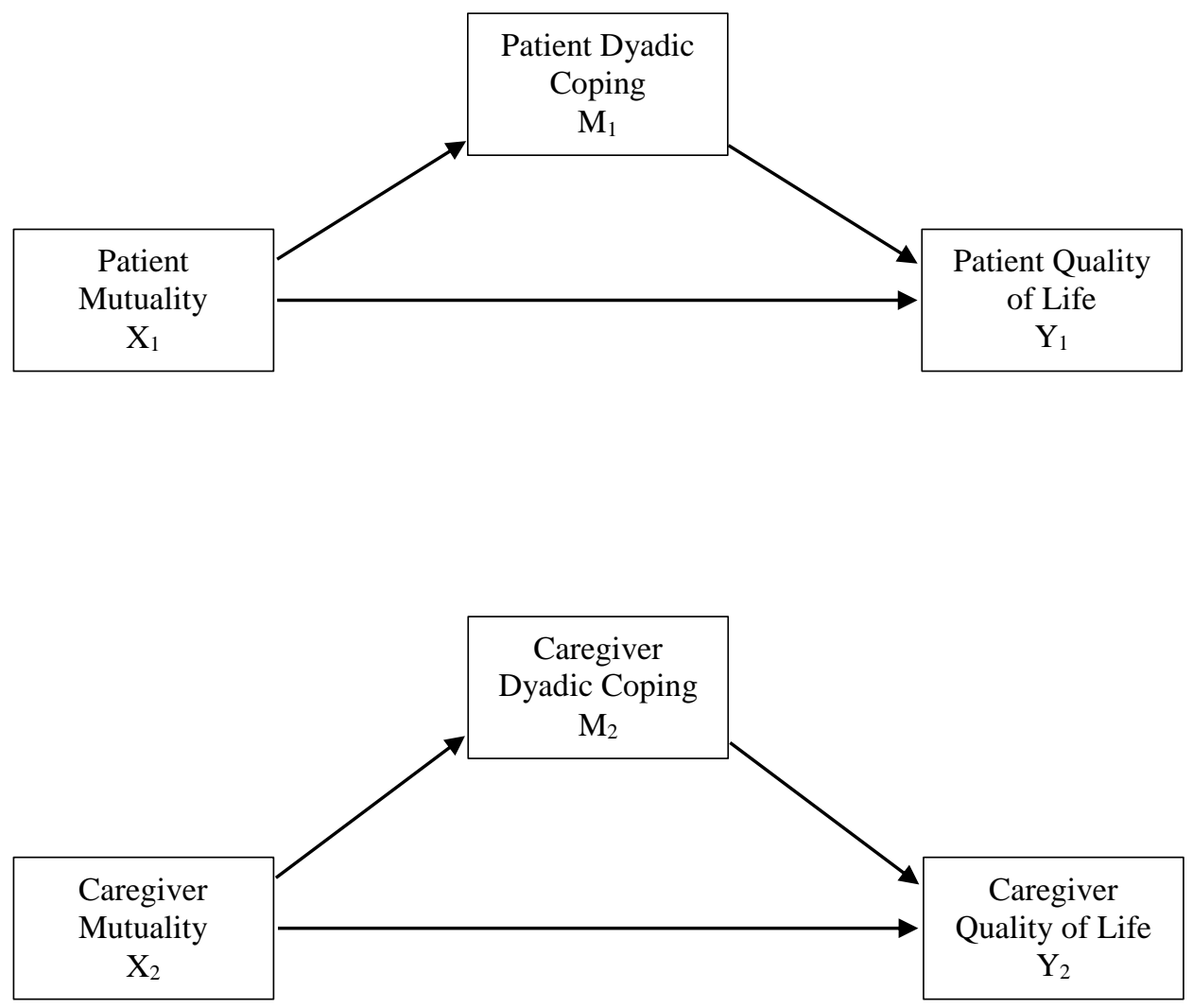


\section{Summary}

This chapter described the methodological plan and analytic strategy for the study of younger couples' experience with the diagnosis of breast cancer, the differences that exist when compared to older ones, and the relationship among mutuality, dyadic coping and quality of life for both members of the dyad in the two groups. The next chapter will provide a detailed description of each analysis as well as their results. 


\section{CHAPTER IV: RESULTS}

This chapter illustrates the results of the present research study. First, data preparation will be reviewed, followed by the description of the sample and correlations among the variables of interest. Then, analyses and results are presented for each research question.

A diagnosis of breast cancer at a younger age is associated with worse outcomes on several psychosocial issues. Despite the evidence that highlights the more detrimental effect of cancer on the couple relationship and the indication available from research on young couples and stress, the attention to the developmental trajectory of dyadic coping over the life-span is a significant gap in the current literature. Hence, the purpose of this study was to investigate how a diagnosis of breast cancer impacts younger patients and partners' quality of life, dyadic coping skills, communication and the expression of emotional support, by comparing them with a group of older dyads. Of particular interest was the consideration of the mechanism through which dyadic coping develops and affects the well-being of patients and partners, especially between the two groups. More specifically, the study attempted to test a conceptual framework inspired by the Relational-Cultural Model of dyadic coping (Kayser et al., 2007), where the relationship between mutuality and quality of life is mediated by dyadic coping. 
Findings related to the following research questions will be described in this chapter:

1. Does a diagnosis of breast cancer affect younger patients and their partners' quality of life, dyadic coping, communication and mutuality more negatively than older couples?

2. How does dyadic coping affect the quality of life of younger patients and partners?

3. How does mutuality affect dyadic coping styles of younger and older breast cancer patients and partners?

4. Does dyadic coping mediate the relationship between mutuality and quality of life of younger and older patients and partners?

This chapter will explain data preparation activities and preliminary analyses, describe the study sample and present the results. The final chapter will discuss the implications of the study, describe its limitations, and outline ideas for future research.

\section{Data Preparation and Preliminary Data Analysis}

After obtaining Institutional Review Board approval, IBM SPSS Statistics 22 was used for data management, data preparation and preliminary data analysis. Descriptive statistics were calculated for all the variables compiling frequency tables, histograms and bar graphs. A Missing Value Analysis (MVA) was conducted on all the variables included in the dataset and revealed that missing data ranged from $0.6 \%$ to $2.3 \%$ of cases on 7 variables (from 1 to a max of 4 cases), suggesting that data were missing at random in the present dataset. Mean substitution was used to handle missing data on the key variables of interest. However, to account for the reduction in the variability of the scores 
often associated with this procedure, mean scores were calculated for each subgroup of the study (i.e.: younger patients, younger partners; older patients and older partners) and the imputation was conducted accordingly (Meyers et al., 2006; Tabachnick \& Fidell, 2007).

Univariate outliers were examined through the SPSS Explore command and the use of Box Plots. Extreme values and outliers were detected for 7 variables. For hostile dyadic coping and avoidance of dyadic coping each reported cases was checked and the values for the extreme cases were left in the database because they truly represented possible scores of the subscale, while the outlier (case 1) was deleted. The same situation was found for the variables total dyadic coping, social and emotional well-being and the total score of the FACT-B, where extreme cases were retained. For the variable mutuality the case 133 was deleted.

Skewness and Kurtosis were investigated to test for normality through SPSS Frequencies and Descriptive analysis (Meyers et al., 2006). Kolmorogov-Smirnov test was also employed to detect departures from normality. Non-normal distributions were detected for the following categorical variables: marital status, race, income, and the mental health variables (history of mental illness treatment, person treated for mental illness, diagnosis of depression, and time of diagnosis for patients). Because of their categorical nature and the data analysis plan, no transformation occurred during the data screening phase. Among the variables of interest, the removal of outliers and the selected missing data imputation procedure improved the overall distribution and none reported problematic Skewness and Kurtosis (Tabachnick \& Fidell, 2007). After inspecting the 
dataset for normality, linearity and homoschedasticity were examined by running bivariate scatterplots and Levene's Test for key study variables.

\section{Description of Sample}

\section{Socio-Demographics, Relational and Clinical Characteristics}

The final sample included 86 dyads of breast cancer patients and their partners. Most of the couples were married (90.7\%) and living together at the time of the study. On average, couples have been together for 20 years $(M=19.58, S D=12.14$, range $1-47)$, although range and standard deviation suggest the existence of quite a variability in the duration of the relationship among participants. Table 6 presents socio-demographic characteristics of the sample, next to indication about relational and clinical variables. The sample consisted entirely of Non-Hispanic White men and women, with the exception of a Hispanic Latino woman and three partners who identified themselves as Hispanic Latino, Black and Asian. Four caregivers did not disclose their racial background.

Breast cancer patients had a mean age of approximately 48 years $(M=48.2$, $\mathrm{SD}=9.83$ ) and most of them had 2 or 3 children. Overall, women were highly educated, as indicated by the fact that $60 \%$ were college graduates, with $30 \%$ having completed a Master's degree, and were employed in professional occupations. Women also indicated a family income above $\$ 90,000$ per year in $57.1 \%$ of the cases, confirming that participants were middle-to-upper middle class and highly educated. Roman Catholicism and Protestantism were the most common religious affiliations reported. Regarding clinical and mental health characteristics, most of women were currently taking medications as part of their treatment and approximately $21 \%$ were receiving 
chemotherapy at the time of the study. Previous diagnosis of mental disorders were limited in the families of the participants. For those who reported a pre-existing mental health diagnosis, parents and siblings were the relatives who reported the highest percentages. Twenty-five percent of women had received treatment for depression, which usually occurred before being diagnosed with breast cancer.

Partners shared similar socio-demographic characteristics. They were on average in their early 50s and most were Non-Hispanic White. Partners were highly educated, with approximately $60.5 \%$ of them being college graduates. They worked in professional settings and approximately $65 \%$ reported an income above $\$ 90,000$, again indicating that participants were middle-to-upper middle class. Also among partners Roman Catholicism and Protestantism were the two largest religious affiliations. Only $20 \%$ reported a previous diagnosis of mental health disorders in the family, which was mostly experienced by siblings and parents. A personal history of depression was disclosed only by 18 of them (19.8\%). No significant differences were detected between patients and partners (see Table 6).

Table 6. Socio-Demographic, Relational and Clinical Characteristics of the Sample

\begin{tabular}{|c|c|c|c|}
\hline Variable & $\begin{array}{c}\text { Breast } \\
\text { Cancer Patients } \\
(\mathrm{N}=\mathbf{8 6})\end{array}$ & $\begin{array}{l}\text { Partners } \\
(\mathrm{N}=86)\end{array}$ & $p$ value \\
\hline $\begin{array}{l}\text { Age } \\
\text { (mean score) }\end{array}$ & $48.21(\mathrm{SD}=9.83)$ & $50.71(\mathrm{SD}=10.83)$ & n.s. \\
\hline $\begin{array}{l}\text { Length of Relationship } \\
\text { (mean score) }\end{array}$ & $19.58(\mathrm{SD}=12.15)$ & $19.58(\mathrm{SD}=12.15)$ & n.s. \\
\hline $\begin{array}{l}\text { Marital Status } \\
\text { Married } \\
\text { Not Married }\end{array}$ & $\begin{array}{c}78(90.7 \%) \\
8(9.3 \%)\end{array}$ & $\begin{array}{c}78(90.7 \%) \\
8(9.3 \%)\end{array}$ & n.s. \\
\hline $\begin{array}{l}\text { Race } \\
\text { White } \\
\text { Black }\end{array}$ & $84(97.7 \%)$ & $\begin{array}{c}79(91.9 \%) \\
1(1.2 \%)\end{array}$ & n.s. \\
\hline
\end{tabular}




\begin{tabular}{|c|c|c|c|}
\hline Asian & - & $1(1.2 \%)$ & \\
\hline Latino & $1(1.2 \%)$ & - & \\
\hline Native American/Indian & - & $1(1.2 \%)$ & \\
\hline Unknown/Other & $1(1.2 \%)$ & $4(4.8 \%)$ & \\
\hline Number of Children & & & n.s \\
\hline 0 & $16(18.6 \%)$ & $13(15.1 \%)$ & \\
\hline 1 & $11(12.8 \%)$ & $11(12.8 \%)$ & \\
\hline 2 & $31(36.0 \%)$ & $32(37.2 \%)$ & \\
\hline 3 & $20(23.3 \%)$ & $22(25.6 \%)$ & \\
\hline 4 & $7(8.1 \%)$ & $7(8.1 \%)$ & \\
\hline 5 & $1(1.2 \%)$ & $1(1.2 \%)$ & \\
\hline Education & & & n.s. \\
\hline Less than High School & - & $2(2.3 \%)$ & \\
\hline High School Graduate & $5(5.8 \%)$ & $4(4.7 \%)$ & \\
\hline High School with some & & & \\
\hline College & $10(11.6 \%)$ & $16(18.6 \%)$ & \\
\hline College Graduate & $27(31.4 \%)$ & $21(24.4 \%)$ & \\
\hline College with some Graduate & $11(12.8 \%)$ & $11(14.0 \%)$ & \\
\hline Hours & & & \\
\hline Master's Degree & $27(31.4 \%)$ & $20(22.1 \%)$ & \\
\hline $\mathrm{PhD}, \mathrm{MD}, \mathrm{JD}$ & $6(7.0 \%)$ & $11(12.8 \%)$ & \\
\hline Other & - & $1(1.2 \%)$ & \\
\hline Occupation & & & n.s. \\
\hline Unskilled Labor & $1(1.2 \%)$ & - & \\
\hline Managerial & $8(9.3 \%)$ & $22(25.6 \%)$ & \\
\hline Homemaker/Parent & $12(14.0 \%)$ & - & \\
\hline Skilled Labor & $3(3.5 \%)$ & $7(8.1 \%)$ & \\
\hline Professional & $53(61.6 \%)$ & $48(55.8 \%)$ & \\
\hline Other & $9(10.5 \%)$ & $9(10.5 \%)$ & \\
\hline Income & & & n.s. \\
\hline$<\$ 10,000$ & $1(1.2 \%)$ & - & \\
\hline$\$ 10,000-29,900$ & $2(2.3 \%)$ & $2(2.3 \%)$ & \\
\hline$\$ 30,000-49,900$ & $9(10.5 \%)$ & $6(7.0 \%)$ & \\
\hline$\$ 50,000-69,900$ & $16(18.6 \%)$ & $12(14.0 \%)$ & \\
\hline$\$ 70,000-89,900$ & $9(10.5 \%)$ & $11(12.8 \%)$ & \\
\hline$<\$ 90,000$ & $49(57.0 \%)$ & $55(64.0 \%)$ & \\
\hline Religious Affiliation & & & n.s. \\
\hline Catholic & $36(41.9 \%)$ & $30(34.9 \%)$ & \\
\hline Protestant & $25(29.1 \%)$ & $27(31.4 \%)$ & \\
\hline Jewish & $10(11.6 \%)$ & $13(15.1 \%)$ & \\
\hline Atheist/Agnostic & $4(4.7 \%)$ & $9(10.5 \%)$ & \\
\hline Other & $11(12.8 \%)$ & $7(8.1 \%)$ & \\
\hline \multicolumn{4}{|l|}{ Current Medications } \\
\hline Yes & $52(61.2 \%)$ & & \\
\hline No & $33(38.8 \%)$ & & \\
\hline \multicolumn{4}{|l|}{ Chemotherapy } \\
\hline Yes & $18(20.9 \%)$ & & \\
\hline No & $66(78.6)$ & & \\
\hline
\end{tabular}




\begin{tabular}{lccc}
\hline $\begin{array}{l}\text { Mental Health Treatment in } \\
\text { the Family }\end{array}$ & & n.s. \\
$\quad$ Yes & $21(24.4 \%)$ & $18(20.9 \%)$ & \\
$\quad$ No & $65(75.6 \%)$ & $68(79.1 \%)$ & \\
Relationship with Person & & & \\
Treated for Mental Health & & & \\
Issues & $6(7.0 \%)$ & $4(4.7 \%)$ & \\
$\quad$ Parent & $6(7.0 \%)$ & $7(8.1 \%)$ & \\
$\quad$ Sibling & $1(1.2 \%)$ & $2(2.3 \%)$ & \\
Grandparent & $6(7.0 \%)$ & $4(4.7 \%)$ & \\
Other & $2(2.3 \%)$ & $2(2.3 \%)$ & \\
Multiple Family Members & $65(75.6 \%)$ & $67(77.9 \%)$ & \\
$\quad$ Not Applicable & & & \\
Treatment for Depression & $22(25.6 \%)$ & $17(19.8 \%)$ & \\
$\quad$ Yes & $64(74.4 \%)$ & $68(79.1 \%)$ & \\
No & & & \\
Timing of Treatment & $20(23.8 \%)$ & & \\
Depression & $2(2.4 \%)$ & & \\
Before Cancer Diagnosis & $1(1.2 \%)$ & & \\
After Cancer Diagnosis & $61(72.6 \%)$ & & \\
Before \& After & & & \\
Not Applicable & & & \\
\hline
\end{tabular}

\section{Variables of Interest}

Table 7 provides an overview of the variables of interest of the present study. In particular, means and variance indicators of dyadic coping, mutuality, communication and quality of life are presented and compared between breast cancer patients and partners. Overall, participants scored in the middle-to-upper range of dyadic coping, mutuality and communication. Low mean scores on hostile and avoidance of dyadic coping confirmed that overall couples were able to implement adaptive coping strategies and behaviors in response to the stress caused by the cancer diagnosis. However, differences existed between patients and caregivers, with women reporting significantly higher stress communication $(p<.001)$, and overall higher scores on total dyadic coping $(p<.01)$. 
Table 7. Sample Characteristics of the Major Study Variables

\begin{tabular}{|c|c|c|c|c|c|c|c|c|c|c|c|c|}
\hline \multirow[b]{2}{*}{ Variable } & \multicolumn{5}{|c|}{ Patients } & \multicolumn{4}{|c|}{ Partners } & \multirow[b]{2}{*}{$\begin{array}{c}\text { Skewness } \\
\text { Kurtosis }\end{array}$} & \multirow[b]{2}{*}{$t$} & \multirow[b]{2}{*}{$p$} \\
\hline & $\mathbf{M}$ & SD & Range & $\begin{array}{c}\text { Potential } \\
\text { Range }\end{array}$ & $\begin{array}{c}\text { Skewness } \\
\text { Kurtosis }\end{array}$ & $\mathbf{M}$ & SD & Range & $\begin{array}{c}\text { Potential } \\
\text { Range }\end{array}$ & & & \\
\hline \multicolumn{13}{|l|}{ Dyadic Coping } \\
\hline Stress & 4.20 & 0.72 & $2.60-5.80$ & $1-6$ & -.02 & 3.48 & 0.74 & $1.80-5.20$ & $1-6$ & .05 & 6.78 & $<.001 * * *$ \\
\hline Communication & & & & & -.57 & & & & & -.08 & & \\
\hline Common & 3.60 & 0.74 & $2.08-5.15$ & $1-6$ & -.02 & 3.58 & 0.63 & $2.31-5.15$ & $1-6$ & .26 & .19 & .84 \\
\hline Dyadic Coping & & & & & -.81 & & & & & -.49 & & \\
\hline Positive & 4.11 & 0.81 & $2.33-5.48$ & $1-6$ & -.24 & 3.98 & 0.67 & $2.72-5.50$ & $1-6$ & .24 & 1.14 & .253 \\
\hline Dyadic Coping & & & & & -.82 & & & & & -.59 & & \\
\hline Hostile & 1.97 & 0.53 & $1.11-3.44$ & $1-6$ & .45 & 1.94 & 0.49 & $1.00-3.67$ & $1-6$ & .50 & .41 & .68 \\
\hline Dyadic Coping & & & & & -.21 & & & & & .74 & & \\
\hline Avoidance of & 2.68 & 0.87 & $1.00-4.67$ & $1-6$ & .25 & 2.59 & 0.71 & $1.00-4.33$ & $1-6$ & -.36 & .79 & .43 \\
\hline Dyadic Coping & & & & & -.09 & & & & & .13 & & \\
\hline Total Dyadic & 173.6 & 25.44 & $98-215$ & 47-282 & -.34 & 165.97 & 19.98 & 119-215 & 47-282 & .17 & 2.15 & $.03 *$ \\
\hline Coping & & & & & -.26 & & & & & -.29 & & \\
\hline Mutuality & 4.42 & 0.66 & $2.82-5.77$ & $1-6$ & -.37 & 4.47 & 0.67 & $3.45-5.59$ & $1-6$ & -.12 & -.38 & .65 \\
\hline & & & & & -.45 & & & & & -.64 & & \\
\hline Communication & 37.86 & 7.08 & $21-50$ & $10-50$ & -.39 & 38.74 & 7.00 & $21-50$ & $10-50$ & -.30 & -.82 & .41 \\
\hline & & & & & -.59 & & & & & -.80 & & \\
\hline \multicolumn{13}{|l|}{ Quality of Life } \\
\hline \multicolumn{13}{|l|}{ Patients } \\
\hline Physical & 20.89 & 5.36 & $7-28$ & $0-28$ & -.86 & & & & & & & \\
\hline Well-being & & & & & -.13 & & & & & & & \\
\hline Social & 22.67 & 3.91 & $12-28$ & $0-28$ & -.69 & & & & & & & \\
\hline Well-being & & & & & -.19 & & & & & & & \\
\hline Emotional & 16.54 & 3.33 & $7-23$ & $0-24$ & -.53 & & & & & & & \\
\hline Well-being & & & & & .05 & & & & & & & \\
\hline Functional & 18.61 & 5.29 & $9-28$ & $0-28$ & -.06 & & & & & & & \\
\hline Well-being & & & & & -.90 & & & & & & & \\
\hline Breast Cancer & 24.41 & 5.10 & $10-36$ & $0-36$ & -.19 & & & & & & & \\
\hline Symptoms & & & & & -.08 & & & & & & & \\
\hline FACT-G & 78.72 & 13.15 & $48-103$ & $0-108$ & -.22 & & & & & & & \\
\hline & & & & & -.62 & & & & & & & \\
\hline FACT-B & 103.13 & 16.80 & $61-137$ & $0-138$ & -.25 & & & & & & & \\
\hline
\end{tabular}




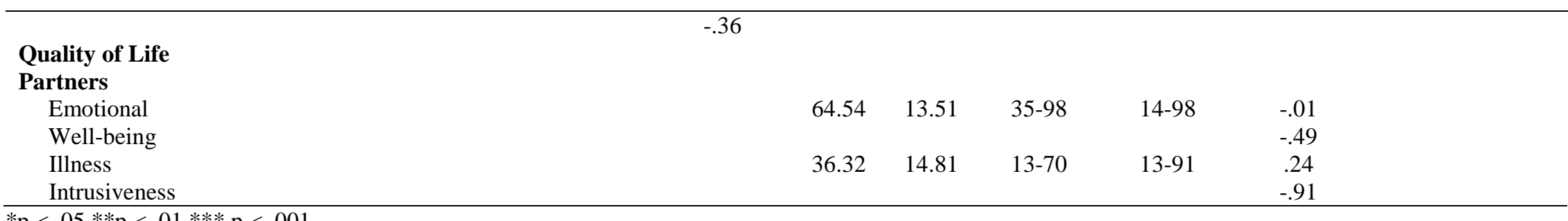

$* \mathrm{p}<.05 * * \mathrm{p}<.01 * * * \mathrm{p}<.001$ 


\section{Younger and Older Couples}

For the purpose of the present investigation, the sample was divided in two groups according to the age of the patient at time of diagnosis. Younger dyads were identified as those where the woman was 45 years old or younger $(n=35)$, while older couples were composed by a woman who was above 46 years of age when diagnosed with breast cancer $(n=51)$ and her partner.

Socio-demographic characteristics and major variables of interest were analyzed for each group, and scores were compared between patients and partners. Table 8 and Table 9 describe the younger sample. Average length of the relationship at time of the survey was of approximately 11 years $(\mathrm{SD}=5.75)$, with all couples living together and almost all of them were married (91.4\%). Overall younger women were in their late thirties $(\mathrm{M}=$ 38.31, $\mathrm{SD}=4.78$ ), highly educated and mostly working as professionals. All patients were Non-Hispanic White and the majority were mothers, with one third of the sample having two children (28.6\%). Half of the women indicated an income higher than $\$ 90,000$ per year. Similar to the overall sample, Catholic, Protestant and Jewish were most common categories selected to describe the religious affiliation of the participants. Only 10 (28.6\%) younger breast cancer patients were currently receiving chemotherapy, while $57.1 \%$ were taking medications as part of their treatment. Considering the mental health variables investigated in the survey, almost $80 \%$ of the respondents denied a previous history of mental disorders in the family and only 6 were treated for depression, which was diagnosed and treated before the cancer diagnosis. Younger partners were on average 40 years old $(\mathrm{SD}=6.65)$ and were mostly Non-Hispanic White, with only 3 of them not disclosing their racial background. In a similar way to what was described earlier for women, they were on average highly educated and could be considered to be middle-to- 
upper middle class. Again, Catholicism, Protestantism and Judaism were the most common religious affiliations of the participants. Most of them denied a history of mental health issues in the family and $20 \%$ were treated for depression.

Table 9 presents an overview of the variables of interest and illustrates the couple's adjustment with cancer within the first three months of diagnosis. Younger couples reported similar scores on common, hostile and avoidance of dyadic coping, with significant differences between the two partners for stress communication $(p<.001)$, and total dyadic coping $(p<.01)$. Women indicated higher scores on hostile and avoidance of dyadic coping, although this difference did not reach statistical significance. Younger couples presented good resources in terms of mutuality and communication, while quality of life variables clearly highlighted the impact cancer had on the well-being of each individual, with younger women reporting affected quality of life in all the subscales of the FACT-B and partners indicating moderately levels of affected well-being and illness intrusiveness.

When considering the older group (Table 10), these couples had been in a relationship for approximately 26 years $(\mathrm{SD}=11.6)$. All of them were living together and the largest majority was married (90.2\%). Older breast cancer patients were in their midfifties $(M=55, S D=5.74)$, were highly educated and mostly working in professional settings (62.7\%). They were all Non-Hispanic White (96.1\%), with the exception of one Latina, and had at least two or more children. Approximately $61 \%$ indicated an income higher than $\$ 90,000$ per year. Catholic, Protestant and Jewish were the most common categories selected to describe the religious affiliation of the participants. Considering the clinical variables investigated, only $8(16.3 \%)$ older patients were currently receiving 
chemotherapy, while the majority $(64.0 \%)$ took medications as part of the cancer treatment. Approximately $75 \%$ of the respondents denied a history of mental health issues in the family and one third (31.4\%) had received treatment for depression, which was diagnosed and treated before the cancer diagnosis. Overall older partners were on average 57 years old $(\mathrm{SD}=6.97)$ and were mostly Non-Hispanic White, with only one Black, one Asian and one Native American participant. Partners were highly educated, as evidenced by the fact that $59 \%$ were college graduates and almost $70 \%$ indicated a yearly income above $\$ 90,000$. Protestantism was the most common religious affiliation reported (39.2\%). Most of them denied a history of mental health issues in the family (78.4\%) and 10 have been or were currently treated for depression. Differences were registered between patients and partners for age and occupation, with partners being significantly older $\left(t_{(100)}=-2.09, p<.05\right)$ and in more managerial and professional positions.

Older dyads are characterized by higher scores on dyadic coping styles like stress communication, positive and common dyadic coping, indicating that the individuals are utilizing individual and relational resources to cope with the stress of the cancer diagnosis (Table 11). Low scores on hostile and avoidance of dyadic coping were common for both partners, although the women's scores tended to be higher. Patients and partners had positive scores on measurements of mutuality and communication, with no significant differences among the two partners. Regarding quality of life, older participants scored high on the subscales that address physical, social and overall quality of life. However, the areas mostly affected by the cancer diagnosis appear to be their emotional and functional well-being. Similarly, older partners presented high levels of emotional wellbeing and limited intrusiveness. 
Table 8. Socio-Demographic, Relational and Clinical Characteristics of Younger Couples

\begin{tabular}{|c|c|c|c|}
\hline Variable & $\begin{array}{c}\text { Younger Breast } \\
\text { Cancer Patients } \\
(\mathbf{N}=35) \\
\end{array}$ & $\begin{array}{c}\text { Younger } \\
\text { Partners } \\
(\mathrm{N}=35) \\
\end{array}$ & $p$ value \\
\hline $\begin{array}{l}\text { Age } \\
\text { (mean score) }\end{array}$ & $38.31(\mathrm{SD}=4.78)$ & $40.6(\mathrm{SD}=6.65)$ & n.s. \\
\hline $\begin{array}{l}\text { Length of Relationship } \\
\text { (mean score) }\end{array}$ & $10.71(\mathrm{SD}=5.75)$ & $10.71(\mathrm{SD}=5.75)$ & n.s. \\
\hline $\begin{array}{l}\text { Marital Status } \\
\text { Married } \\
\text { Not Married }\end{array}$ & $\begin{array}{c}32(91.4 \%) \\
3(8.6 \%)\end{array}$ & $\begin{array}{c}32(91.4 \%) \\
3(8.6 \%)\end{array}$ & \\
\hline $\begin{array}{l}\text { Race } \\
\text { Non-Hispanic White } \\
\text { Black } \\
\text { Asian } \\
\text { Latino } \\
\text { Native American/Indian } \\
\text { Unknown/Other }\end{array}$ & $\begin{array}{c}35(100 \%) \\
- \\
- \\
- \\
- \\
-\end{array}$ & $\begin{array}{c}32(91.4 \%) \\
- \\
- \\
- \\
- \\
3(8.65)\end{array}$ & n.s. \\
\hline $\begin{array}{l}\text { Number of Children } \\
0 \\
1 \\
2 \\
3 \\
4 \\
5\end{array}$ & $\begin{array}{c}8(22.9 \%) \\
7(20.0 \%) \\
10(28.6 \%) \\
8(22.9 \%) \\
2(5.7 \%) \\
-\end{array}$ & $\begin{array}{c}9(25.7 \%) \\
6(17.15) \\
10(28.6 \%) \\
8(22.9 \%) \\
2(5.7 \%) \\
-\end{array}$ & n.s. \\
\hline $\begin{array}{l}\text { Education } 21 \\
\text { Less than High School } \\
\text { High School Graduate } \\
\text { High School with some College } \\
\text { College Graduate } \\
\text { College with some Graduate } \\
\text { Hours } \\
\text { Master's Degree } \\
\text { PhD, MD, JD } \\
\text { Other }\end{array}$ & $\begin{array}{c}- \\
1(2.9 \%) \\
4(11.4 \%) \\
14(40.0 \%) \\
3(8.6 \%) \\
9(25.7 \%) \\
4(11.4 \%) \\
-\end{array}$ & $\begin{array}{c}2(5.7 \%) \\
2(5.7 \%) \\
6(17.1 \%) \\
10(28.6 \%) \\
7(20 \%) \\
5(14.3 \%) \\
2(5.7 \%) \\
1(2.9 \%)\end{array}$ & n.s. \\
\hline $\begin{array}{l}\text { Occupation }^{22} \\
\text { Unskilled Labor } \\
\text { Managerial } \\
\text { Homemaker/Parent } \\
\text { Skilled Labor } \\
\text { Professional } \\
\text { Other }\end{array}$ & $\begin{array}{c}- \\
5(14.3 \%) \\
5(14.3 \%) \\
2(5.7 \%) \\
21(60.0 \%) \\
2(5.7 \%)\end{array}$ & $\begin{array}{c}- \\
6(17.1 \%) \\
- \\
5(14.3 \%) \\
21(60.0 \%) \\
3(8.6 \%)\end{array}$ & n.s. \\
\hline
\end{tabular}

\footnotetext{
${ }^{21}$ Non-significant differences are detected also when the variable is recoded in 2 categories, $1=$ High School, and 2=College graduate

${ }^{22}$ Non-significant differences are detected also when the variable is recoded in 2 categories. Unskilled labor, Homemaker, and other were recoded as 1, managerial, skilled labor and professional were recoded as 2. The Fisher's Exact Test indicates a 2-sided significance of .31.
} 


\begin{tabular}{|c|c|c|c|}
\hline Income & & & n.s. \\
\hline$<\$ 10,000$ & - & - & \\
\hline$\$ 10,000-29,900$ & $1(2.9 \%)$ & $1(2.9 \%)$ & \\
\hline$\$ 30,000-49,900$ & $2(5.7 \%)$ & $1(2.9 \%)$ & \\
\hline$\$ 50,000-69,900$ & $6(17.1 \%)$ & $6(17.1 \%)$ & \\
\hline$\$ 70,000-89,900$ & $8(22.9 \%)$ & $7(20.0 \%)$ & \\
\hline$<\$ 90,000$ & $18(51.4 \%)$ & $20(57.1 \%)$ & \\
\hline Religious Affiliation & & & n.s. \\
\hline Catholic & $17(48.6 \%)$ & $15(42.9 \%)$ & \\
\hline Protestant & $8(22.9 \%)$ & $7(20.0 \%)$ & \\
\hline Jewish & $3(8.6 \%)$ & $3(8.6 \%)$ & \\
\hline Atheist/Agnostic & $1(2.9 \%)$ & $5(14.3 \%)$ & \\
\hline Other & $6(17.1 \%)$ & $5(14.3 \%)$ & \\
\hline \multicolumn{4}{|l|}{ Current Medications } \\
\hline Yes & $20(57.1 \%)$ & & \\
\hline No & $15(42.9 \%)$ & & \\
\hline \multicolumn{4}{|l|}{ Chemotherapy } \\
\hline Yes & $10(28.6 \%)$ & & \\
\hline No & $25(71.4 \%)$ & & \\
\hline \multicolumn{4}{|l|}{ Mental Health Treatment in the } \\
\hline \multicolumn{4}{|l|}{ Family } \\
\hline Yes & $8(22.95)$ & $7(20.0 \%)$ & \\
\hline No & $27(77.1 \%)$ & $28(80.0 \%)$ & \\
\hline $\begin{array}{l}\text { Relationship with Person Treated } \\
\text { for Mental Health Issues }\end{array}$ & & & n.s. \\
\hline Parent & $1(2.9 \%)$ & - & \\
\hline Sibling & $2(5.7 \%)$ & $3(8.6 \%)$ & \\
\hline Grandparent & $1(2.9 \%)$ & $1(2.9 \%)$ & \\
\hline Other & $3(8.6 \%)$ & $4(11.4 \%)$ & \\
\hline Multiple Family Members & $1(2.9 \%)$ & - & \\
\hline Not Applicable & $27(77.1 \%)$ & $27(77.1 \%)$ & \\
\hline Treatment for Depression & & & n.s. \\
\hline Yes & $6(17.1 \%)$ & $7(20.0 \%)$ & \\
\hline No & $29(82.9 \%)$ & $28(80.0 \%)$ & \\
\hline \multicolumn{4}{|l|}{ Timing of Treatment Depression } \\
\hline Before Cancer Diagnosis & $6(17.1 \%)$ & & \\
\hline After Cancer Diagnosis & - & & \\
\hline Before \& After & - & & \\
\hline Not Applicable & $29(82.9 \%)$ & & \\
\hline
\end{tabular}


Table 9. Sample Characteristics of the Major Study Variables for Younger Couples

\begin{tabular}{|c|c|c|c|c|c|c|c|c|c|c|c|c|}
\hline \multirow[b]{2}{*}{ Variables } & \multicolumn{5}{|c|}{ Patients } & \multicolumn{5}{|c|}{ Partners } & \multirow[b]{2}{*}{$t$} & \multirow[b]{2}{*}{$p$} \\
\hline & $\mathbf{M}$ & SD & Range & $\begin{array}{c}\text { Potential } \\
\text { Range }\end{array}$ & $\begin{array}{l}\text { Skewness } \\
\text { Kurtosis }\end{array}$ & $\mathbf{M}$ & SD & Range & $\begin{array}{c}\text { Potential } \\
\text { Range }\end{array}$ & $\begin{array}{l}\text { Skewness } \\
\text { Kurtosis }\end{array}$ & & \\
\hline $\begin{array}{l}\text { Dyadic Coping } \\
\text { Stress } \\
\text { Communication }\end{array}$ & 4.14 & 0.79 & $\begin{array}{c}2.60- \\
5.80\end{array}$ & $1-6$ & $\begin{array}{c}.20 \\
-.52\end{array}$ & 3.37 & .70 & $1.80-4.80$ & $1-6$ & $\begin{array}{c}-.05 \\
.23\end{array}$ & 4.36 & $<.001 * * *$ \\
\hline $\begin{array}{l}\text { Common Dyadic } \\
\text { Coping }\end{array}$ & 3.53 & 0.67 & $\begin{array}{l}2.08- \\
5.08\end{array}$ & $1-6$ & $\begin{array}{l}.26 \\
-.29\end{array}$ & 3.51 & .52 & $2.46-4.46$ & $1-6$ & $\begin{array}{l}.02 \\
-.77\end{array}$ & 0.15 & .88 \\
\hline $\begin{array}{l}\text { Positive Dyadic } \\
\text { Coping }\end{array}$ & 4.08 & 0.82 & $\begin{array}{l}2.59- \\
5.48\end{array}$ & $1-6$ & $\begin{array}{l}.11 \\
-.93\end{array}$ & 3.88 & .56 & $2.90-5.34$ & $1-6$ & $\begin{array}{l}.66 \\
.65\end{array}$ & 1.19 & .237 \\
\hline $\begin{array}{l}\text { Hostile Dyadic } \\
\text { Coping }\end{array}$ & 2.07 & 0.57 & $\begin{array}{l}1.11- \\
3.44\end{array}$ & $1-6$ & $\begin{array}{l}.45 \\
-.21\end{array}$ & 2.13 & .45 & $1.11-3.00$ & $1-6$ & $\begin{array}{l}-.39 \\
-.08\end{array}$ & -0.46 & .64 \\
\hline $\begin{array}{l}\text { Avoidance of } \\
\text { Dyadic Coping }\end{array}$ & 2.79 & 0.89 & $\begin{array}{l}1.00- \\
4.67\end{array}$ & $1-6$ & $\begin{array}{l}.23 \\
-.49\end{array}$ & 2.69 & .75 & $1-4$ & $1-6$ & $\begin{array}{r}-.69 \\
.24\end{array}$ & 0.50 & .61 \\
\hline $\begin{array}{l}\text { Total Dyadic } \\
\text { Coping }\end{array}$ & 175.12 & 23.74 & 131-217 & 47-282 & $\begin{array}{l}.25 \\
-.94\end{array}$ & 163.68 & 15.99 & 136-201 & $47-282$ & $\begin{array}{l}.52 \\
.26\end{array}$ & 2.36 & $.02 *$ \\
\hline Mutuality & 4.38 & 0.70 & $\begin{array}{l}2.86- \\
5.55\end{array}$ & $1-6$ & $\begin{array}{l}-.44 \\
-.63\end{array}$ & 4.45 & 0.48 & $3.45-5.41$ & $1-6$ & $\begin{array}{l}-.26 \\
-.40\end{array}$ & -0.53 & .59 \\
\hline Communication & 38.34 & 7.01 & $25-50$ & $10-50$ & $\begin{array}{l}-.37 \\
-.84\end{array}$ & 38.40 & 7.17 & $21-50$ & $10-50$ & $\begin{array}{l}-.44 \\
-.43\end{array}$ & -0.03 & .97 \\
\hline \multicolumn{13}{|l|}{ Quality of Life } \\
\hline $\begin{array}{l}\text { Physical } \\
\text { Well-being }\end{array}$ & 18.51 & 6.15 & $7-28$ & $0-28$ & $\begin{array}{l}-.18 \\
-.96\end{array}$ & & & & & & & \\
\hline $\begin{array}{l}\text { Social } \\
\text { Well-being }\end{array}$ & 21.52 & 4.07 & $14-28$ & $0-28$ & $\begin{array}{l}-.17 \\
-.98\end{array}$ & & & & & & & \\
\hline $\begin{array}{l}\text { Emotional } \\
\text { Well-being }\end{array}$ & 15.14 & 3.65 & $7-21$ & $0-24$ & $\begin{array}{l}-.39 \\
-.55\end{array}$ & & & & & & & \\
\hline $\begin{array}{l}\text { Functional } \\
\text { Well-being }\end{array}$ & 17.60 & 5.65 & $9-28$ & $0-28$ & $\begin{array}{c}.44 \\
-.87\end{array}$ & & & & & & & \\
\hline $\begin{array}{l}\text { Breast Cancer } \\
\text { Symptoms }\end{array}$ & 22.58 & 5.70 & $10-36$ & $0-36$ & $\begin{array}{l}.26 \\
.02\end{array}$ & & & & & & & \\
\hline FACT-G & 72.77 & 14.50 & $43-103$ & $0-108$ & $\begin{array}{l}.41 \\
-.62\end{array}$ & & & & & & & \\
\hline FACT-B & 95.33 & 19.35 & $60-136$ & $0-138$ & .45 & & & & & & & \\
\hline
\end{tabular}




\begin{tabular}{|c|c|c|c|c|c|c|}
\hline \multirow{2}{*}{ Quality of Life } & -.49 & & & & & \\
\hline & & & & & & \\
\hline Partners & & & & & & \\
\hline Emotional & & 58.83 & 12.91 & $35-84$ & $14-98$ & .09 \\
\hline Well-being & & & & & & -.95 \\
\hline Illness & & 43.06 & 14.12 & $16-70$ & $13-91$ & -.27 \\
\hline Intrusiveness & & & & & & -.74 \\
\hline
\end{tabular}


Table 10. Socio-Demographic, Relational and Clinical Characteristics of Older Couples

\begin{tabular}{|c|c|c|c|}
\hline Variable & $\begin{array}{c}\text { Older Breast } \\
\text { Cancer Patients } \\
(\mathrm{N}=\mathbf{5 1})\end{array}$ & $\begin{array}{c}\text { Older } \\
\text { Partners } \\
(\mathrm{N}=\mathbf{5 1})\end{array}$ & $p$ value \\
\hline Age & & & $<.05$ \\
\hline (mean score) & $55.00(\mathrm{SD}=5.74)$ & $57.65(\mathrm{SD}=6.97)$ & \\
\hline Length of Relationship & & & n.s. \\
\hline (mean score) & $25.66(\mathrm{SD}=11.64)$ & $25.66(\mathrm{SD}=11.64)$ & \\
\hline Marital Status & & & n.s. \\
\hline Married & $46(90.2 \%)$ & $46(90.2 \%)$ & \\
\hline Not Married & $5(9.8 \%)$ & $5(9.8 \%)$ & \\
\hline Race & & & n.s. \\
\hline Non-Hispanic White & $49(96.1 \%)$ & $47(92.2 \%)$ & \\
\hline Black & - & $1(2.0 \%)$ & \\
\hline Asian & - & $1(2.0 \%)$ & \\
\hline Latino & $1(2.0 \%)$ & - & \\
\hline Native American/Indian & - & $1(2.0 \%)$ & \\
\hline Unknown/Other & $1(2.0 \%)$ & $1(2.0 \%)$ & \\
\hline Number of Children & & & n.s. \\
\hline 0 & $8(15.75)$ & $4(7.8 \%)$ & \\
\hline 1 & $4(7.8 \%)$ & $5(9.8 \%)$ & \\
\hline 2 & $21(41.2 \%)$ & $22(43.1 \%)$ & \\
\hline 3 & $12(23.5 \%)$ & $14(27.5 \%)$ & \\
\hline 4 & $5(9.8 \%)$ & $5(9.8 \%)$ & \\
\hline 5 & $1(2.0 \%)$ & $1(2.0 \%)$ & \\
\hline Education 23 & & & n.s. \\
\hline Less than High School & - & - & \\
\hline High School Graduate & $4(7.8 \%)$ & $2(3.9 \%)$ & \\
\hline High School with some College & $6(11.6 \%)$ & $10(19.6 \%)$ & \\
\hline College Graduate & $13(25.5 \%)$ & $11(21.6 \%)$ & \\
\hline College with some Graduate & & & \\
\hline Hours & $8(15.7 \%)$ & $4(7.8 \%)$ & \\
\hline Master's Degree & $18(35.3 \%)$ & $15(29.4 \%)$ & \\
\hline $\mathrm{PhD}, \mathrm{MD}, \mathrm{JD}$ & $2(3.9 \%)$ & $9(17.6 \%)$ & \\
\hline Other & - & - & \\
\hline Occupation $^{24}$ & & & n.s. \\
\hline Unskilled Labor & $1(2.0 \%)$ & - & \\
\hline Managerial & $3(5.9 \%)$ & $16(31.4 \%)$ & \\
\hline Homemaker/Parent & $7(13.7 \%)$ & - & \\
\hline Skilled Labor & $1(2.0 \%)$ & $2(3.9 \%)$ & \\
\hline Professional & $32(62.7 \%)$ & $27(52.9 \%)$ & \\
\hline Other & $7(13.7 \%)$ & $6(11.8 \%)$ & \\
\hline
\end{tabular}

${ }^{23}$ Non-significant differences are detected also when the variable is recoded in 2 categories, $1=$ High School graduate, and $2=$ College graduate.

${ }^{24}$ To conduct this chi-square analysis the variable Occupation was recoded in two categories to highlight differences between unskilled and skilled labor. Unskilled labor, homemaker, and other occupations were recoded as 1, managerial, skilled labor and professional were recoded as 2. The Fisher's Exact Test indicates a 2-sided significance of .048. 


\begin{tabular}{|c|c|c|c|}
\hline Income & & & n.s. \\
\hline$<\$ 10,000$ & $1(2.0 \%)$ & - & \\
\hline$\$ 10,000-29,900$ & $1(2.0 \%)$ & $1(2.0 \%)$ & \\
\hline$\$ 30,000-49,900$ & $7(13.7 \%)$ & $5(9.85)$ & \\
\hline$\$ 50,000-69,900$ & $10(19.6 \%)$ & $6(11.8 \%)$ & \\
\hline$\$ 70,000-89,900$ & $1(2.0 \%)$ & $4(7.8 \%)$ & \\
\hline$<\$ 90,000$ & $31(60.8 \%)$ & $35(68.6 \%$ & \\
\hline Religious Affiliation & & & n.s. \\
\hline Catholic & $19(37.3 \%)$ & $15(29.4 \%)$ & \\
\hline Protestant & $17(33.3 \%)$ & $20(39.2 \%)$ & \\
\hline Jewish & $7(13.7 \%)$ & $10(19.6 \%)$ & \\
\hline Atheist/Agnostic & $3(5.9 \%)$ & $4(7.8 \%)$ & \\
\hline Other & $5(9.8 \%)$ & $2(3.9 \%)$ & \\
\hline \multicolumn{4}{|l|}{ Current Medications } \\
\hline Yes & $32(64.0 \%)$ & & \\
\hline No & $18(36.0 \%)$ & & \\
\hline \multicolumn{4}{|l|}{ Chemotherapy } \\
\hline Yes & $8(16.35)$ & & \\
\hline No & $41(83.7 \%)$ & & \\
\hline Mental Health Treatment in the & & & n.s. \\
\hline \multicolumn{4}{|l|}{ Family } \\
\hline Yes & $13(25.5 \%)$ & $11(21.6 \%)$ & \\
\hline No & $38(74.5 \%)$ & $40(78.4 \%)$ & \\
\hline $\begin{array}{l}\text { Relationship with Person Treated } \\
\text { for Mental Health Issues }\end{array}$ & \multicolumn{2}{|c|}{ for Mental Health Issues } & n.s. \\
\hline Parent & $5(9.85)$ & $4(7.8 \%)$ & \\
\hline Sibling & $4(7.85)$ & $4(7.8 \%)$ & \\
\hline Grandparent & - & $1(2.0 \%)$ & \\
\hline Other & $3(5.9 \%)$ & - & \\
\hline Multiple Family Members & $1(2.0 \%)$ & $2(3.9 \%)$ & \\
\hline Not Applicable & $38(74.5 \%)$ & $40(78.4 \%)$ & \\
\hline Treatment for Depression & & & n.s. \\
\hline Yes & $16(31.4 \%)$ & $10(19.6 \%)$ & \\
\hline No & $35(68.6 \%)$ & $40(78.4 \%)$ & \\
\hline \multicolumn{4}{|l|}{ Timing of Treatment Depression } \\
\hline Before Cancer Diagnosis & $14(28.6 \%)$ & & \\
\hline After Cancer Diagnosis & $2(4.15)$ & & \\
\hline Before \& After & $1(2.05)$ & & \\
\hline Not Applicable & $32(62.7 \%)$ & & \\
\hline
\end{tabular}


Table 11. Sample Characteristics of the Major Study Variables for Older Couples

\begin{tabular}{|c|c|c|c|c|c|c|c|c|c|c|c|c|}
\hline \multirow[b]{2}{*}{ Variables } & \multicolumn{4}{|c|}{ Patients } & \multicolumn{6}{|c|}{ Partners } & \multirow[b]{2}{*}{$t$} & \multirow[b]{2}{*}{$p$} \\
\hline & $\mathbf{M}$ & SD & Range & $\begin{array}{c}\text { Potential } \\
\text { Range }\end{array}$ & $\begin{array}{c}\text { Skewness } \\
\text { Kurtosis }\end{array}$ & $\mathbf{M}$ & SD & Range & $\begin{array}{c}\text { Potential } \\
\text { Range }\end{array}$ & $\begin{array}{c}\text { Skewness } \\
\text { Kurtosis }\end{array}$ & & \\
\hline \multicolumn{13}{|l|}{ Dyadic Coping } \\
\hline Stress & 4.26 & .67 & $2.60-5.40$ & $1-6$ & -.18 & 3.49 & 0.76 & $1.80-5.20$ & $1-6$ & .06 & 5.37 & $<.001 * * *$ \\
\hline Communication & & & & & -.54 & & & & & -.19 & & \\
\hline Common Dyadic & 3.65 & 0.79 & $2.31-5.15$ & $1-6$ & -.19 & 3.63 & 0.69 & $2.31-5.15$ & $1-6$ & .22 & .133 & .895 \\
\hline Coping & & & & & -.95 & & & & & -.69 & & \\
\hline Positive Dyadic & 4.12 & 0.80 & $2.33-5.48$ & $1-6$ & -.41 & 4.08 & 0.74 & $2.72-5.52$ & $1-6$ & -.02 & .234 & .815 \\
\hline Coping & & & & & -.80 & & & & & -.88 & & \\
\hline Hostile Dyadic & 1.90 & 0.49 & $1.11-3.00$ & $1-6$ & .34 & 1.80 & 0.47 & $1-3.67$ & $1-6$ & .24 & 1.29 & .198 \\
\hline Coping & & & & & -.43 & & & & & .63 & & \\
\hline Avoidance of & 2.61 & 0.85 & $1-4.67$ & $1-6$ & .25 & 2.51 & 0.68 & $1-4.33$ & $1-6$ & -.16 & .638 & .525 \\
\hline Dyadic Coping & & & & & .65 & & & & & .47 & & \\
\hline Total Dyadic & 172.52 & 26.72 & $98-221$ & $47-282$ & -.60 & 167.54 & 23.51 & $119-215$ & $47-282$ & -.02 & 1.003 & .318 \\
\hline Coping & & & & & -.09 & & & & & -.69 & & \\
\hline Mutuality & 4.46 & 0.63 & $2.82-5.77$ & $1-6$ & -.29 & 4.48 & 0.78 & $1.00-5.59$ & $1-6$ & -.47 & -.182 & .856 \\
\hline & & & & & -.30 & & & & & -.37 & & \\
\hline Communication & 37.52 & 7.17 & $21-50$ & $10-50$ & -.40 & 38.98 & 6.95 & $25-50$ & $10-50$ & -.20 & -1.04 & .302 \\
\hline & & & & & -.40 & & & & & -.17 & & \\
\hline \multicolumn{13}{|l|}{ Quality of Life } \\
\hline \multicolumn{13}{|l|}{ Patients } \\
\hline Physical & 22.52 & 4.05 & $10-28$ & $0-28$ & .33 & & & & & & & \\
\hline Well-being & & & & & .83 & & & & & & & \\
\hline Social & 23.47 & 3.62 & $12-28$ & $0-28$ & .15 & & & & & & & \\
\hline Well-being & & & & & .66 & & & & & & & \\
\hline Emotional & 17.51 & 2.73 & $12-23$ & $0-24$ & -.15 & & & & & & & \\
\hline Well-being & & & & & -.54 & & & & & & & \\
\hline Functional & 19.30 & 4.97 & $9-27$ & $0-28$ & -.43 & & & & & & & \\
\hline Well-being & & & & & -.68 & & & & & & & \\
\hline Breast Cancer & 25.66 & 4.26 & $15-35$ & $0-36$ & -.25 & & & & & & & \\
\hline Symptoms & & & & & .07 & & & & & & & \\
\hline FACT-G & 82.79 & 11.63 & $50-103$ & $0-108$ & -.33 & & & & & & & \\
\hline & & & & & -.02 & & & & & & & \\
\hline FACT-B & 108.46 & 12.36 & $70-137$ & $0-138$ & -.22 & & & & & & & \\
\hline
\end{tabular}




\begin{tabular}{lrrrr}
\hline Quality of Life & .33 & & & \\
Partners & & & \\
$\quad$ Emotional & 68.47 & 12.58 & $40-98$ & $14-98$ \\
$\quad$ Well-being & 31.70 & 13.56 & $13-64$ & $13-91$ \\
$\quad$ Illness & & -.02 & .61 \\
$\quad$ Intrusiveness & & & .65 \\
\hline $\mathrm{p}<.05 * * \mathrm{p}<.01 * * * \mathrm{p}<.001$ & &
\end{tabular}




\section{Correlations}

\section{Bivariate Analyses: Patients}

As a pre-requisite for later analyses and to investigate multicollinearity, bivariate correlations between the major study variables of patients and partners, as well as the relationships between these variables and socio-demographic, clinical, relational and mental health variables were examined ${ }^{25}$.

\section{Correlations among the Major Study Variables}

Moderate positive correlations were found between self-reported levels of mutuality and stess communication $(r=.36, p<.01)$, and common dyadic coping $(r=.42, p<.001)$. Additionally, breast cancer patients' mutuality in their relationship with the partner was associated with higher ability to communicate their stress and to engage in a coordinate and symmetrical type of dyadic coping ( $r=.51, p<.001)$. In contrast, women who presented higher scores on negative coping styles were characterized by reduced levels of perceived mutuality (Hostile Dyadic Coping: $r=-.44, p<.001$; Avoidance of Dyadic Coping $r=-.37, p<.001)$. In the same direction were the correlations between dyadic coping and communication, with women higher on adaptive dyadic coping styles reporting higher levels of communication (Stress Communication: $r=.33, p<.01$; Common Dyadic Coping: $r=.39, p<.001$; Positive Dyadic Coping: $r=.47, p<.001$ ). Similarly, positive associations were revealed between adaptive dyadic coping styles (Stress Communication, Common and Positive Dyadic Coping) and higher social wellbeing, while a negative correlation was found between total dyadic coping and emotional well-being $(r=-.21, p<.05)$. Higher impact of breast cancer symptoms and additional

\footnotetext{
${ }^{25}$ To facilitate the review of the chapter, all the tables for these analyses have been placed in Appendix B and the coefficients have been reported in the text.
} 
concerns were experienced by patients who showed high hostile dyadic coping $(r=-.22$, $p<.05)$. This maladptive coping style was also associated to lower quality of life, as evidenced by a negative correlation with the FACT-G and FACT-B total scores $(r=-.32$, $p<.05, r=-.27, p<.01)$. Among women in our sample, higher self-reported levels of mutuality were associated with higher social and functional well-being $(r=.62, p<.001$; $r=.24, p<.05)$, lower impact of breast cancer symptoms $(r=.12, p<.05)$ and overall higher quality of life (FACT-G: $r=.30, p<.01$, FACT-B: $r=.27, p<.05$ ).

\section{Correlations between Socio-Demographic Characteristics and Major Study Variables}

Older women tended to have more children $(r=.30, p<.01)$, and their number was negatively associated to the level of education of the individual $(r=-.25, p<.05)$. As expected, an association existed between education and occupation, with more educated women working as professionals $(r=.30, p<.01)$. Age was positively associated with physical $(r=.27, p<.05)$, emotional well-being $(r=.32, p<.01)$, and overall higher levels of quality of life (FACT-G: $r=.28, p<.05$; FACT-B: $r=.28, p<.05$ ).

\section{Correlations Relational Variables and Major Study Variables}

Cohabitation was excluded from these analyses because it was constant across the sample; all couples were living together at the time of the study. Moderate positive correlations were identified between length of relationship and physical $(r=.25, p<.05)$, social $(r=.21, p<.05)$ and emotional well-being $(r=.28, p<.05)$. These positive associations were confirmed also when the total score on the FACT-B and FACT-G were included in the analysis (FACT-G: $r=.31, p<.01$; FACT-B: $r=.28, p<.05$ ). Hence, in the present sample of breast cancer patients, the duration of the relationship was associated 
with higher physical, social and emotional well-being as well as to overall higher quality of life.

\section{Correlations Clinical Variables and Major Study Variables}

Bivariate associations between clinical variables and the variables of interest were examined. Taking medications as part of the cancer treatment was associated with lower physical well-being $(r=-.32, p<.01)$ and higher scores on hostile dyadic coping $(r=.26$, $p<.05)$. Women who were undergoing chemotherapy reported lower scores on physical well-being $(r=-.33, p<.01)$. Similarly, negative moderate correlations were revealed between both these two variables and lower levels of functional well-being, higher impact of breast cancer symptoms and lower overall quality of life scores on the FACT-B $(r=-.30, p<.01 ; r=-.31, p<.05$, respectively $)$.

\section{Correlations Mental Health and Major Study Variables}

Finally, the association between mental health variables and dyadic coping, mutuality, communication and quality of life was examined. A moderate positive correlation was found between a family history for mental health treatment, and patients' treatment for depression $(r=.35, p<.05)$. Furthermore, present analysis highlighted that for patients who have received treatment for depression, lower scores were found for functional well-being $(r=-.27, p<.05)$ and on the FACT-G subscale $(r=-.22, p<.05)$.

\section{Bivariate Analyses: Partners}

\section{Correlations among the Major Study Variables}

Findings from the bivariate analyses on partners revealed numerous significant relationships among the study's variables of interest. Men's perception of mutuality in their relationship was positively correlated with dyadic coping styles like stress 
communication $(r=.30, p<.01)$, common dyadic coping $(r=.75, p<.001)$, and positive dyadic coping $(r=.76, p<.001)$, while higher levels of perceived mutuality were inversely associated with negative coping behaviors, namely hostile and avoidance of dyadic coping $(r=-.57 ; r=-.58, p<.001)$. Similarly, communication scores correlated positively with adaptive dyadic coping style (stress communication: $r=.21, p<.05$;

common dyadic coping: $r=.67, p<.001$; positive dyadic coping: $r=.65, p<.001$ ) and negatively with maladaptive coping styles (hostile dyadic coping: $r=-.54, p<.001$; avoidance of dyadic coping: $r=-.63, p<.001)$. Significant correlations were also identified between emotional well-being and illness intrusiveness, with hostile dyadic coping negatively associated with emotional well-being $(r=-.31, p<.01)$ and greater illness intrusiveness of the woman's cancer $(r=.37, p<.001)$. A similar association was identified for avoidance of dyadic coping and reduced emotional well-being $(r=-.21$, $p<.05)$.

\section{Correlations between Socio-Demographic Characteristics and Major Study Variables}

Results of bivariate analyses between partners' socio-demographic characteristics and the variables of interest indicate that higher emotional well-being and lower illness intrusiveness are associated with age. In the present sample age was associated with higher quality of life and reduced impact of the illness demands $(r=.34, p<.01$ and $r=-$ $.42, p<.001)$. Additionally, age was associated inversely with negative coping behaviors, like hostile dyadic coping $(r=-.24, p<.05)$. However, no other socio-demographic variables were apparently correlated with dyadic coping. Higher intrusiveness was related to the partner's occupation, with higher demands registered for individuals working as professionals $(r=.24, p<.05)$. Individuals reporting greater income and affiliated with a 
religion had more numerous families $(r=.22, p<.05$ and $r=-.24, p<.05)$. Higher stress communication behaviors were registered for highly educated partners $(r=.21, p<.05)$. Correlations between Relational Variables and Major Study Variables

With regard to the relational variables, the duration of the relationship was associated with lower scores of hostile dyadic coping $(r=-.27, p<.05)$, higher emotional well-being $(r=.26, p<.05)$ and reduced illness intrusiveness $(r=-.24, p<.05)$.

\section{Correlations between Mental Health and Major Study Variables}

Bivariate correlations between mental health variables and the main variables of interest indicate that partners diagnosed with depression utilized fewer positive coping strategies such as common dyadic coping $(r=-.27, p<.05)$, positive dyadic coping $(r=-$ $.24, p<.05)$, while also presenting lower mutuality $(r=-.28, p<.01)$ and communication scores $(r=-.24, p<.05)$. 
Specific Aim 1: To describe differences between younger and older couples on Quality of Life, Dyadic Coping, Communication and Mutuality

Hypothesis 1.1: Younger patients with breast cancer will report lower quality of life, mutuality, communication, and higher negative dyadic coping styles compared to older breast cancer patients

An independent- samples $t$ test compared the mean scores of quality of life, mutuality, communication and dyadic coping styles of younger and older breast cancer patients. Results indicate that no differences existed in the present sample for mutuality, communication and dyadic coping styles, suggesting that younger and older patients present similar coping skills (Table 12). However, younger women did report worse quality of life than their older counterpart. Significant mean differences were identified for physical $\left(t_{(53)}=-3.38, p<.01\right)$, social $\left(t_{(84)}=-2.33, p<.05\right)$ and emotional well-being $\left(t_{(59)}=-3.26, p<.01\right)$, next to higher impact of breast cancer symptoms $\left(t_{(84)}=-2.86, p<\right.$ $.01)$. These results were also confirmed when the total scores of the FACT-G $\left(t_{(57)}=-\right.$ $3.51, p<.01)$ and FACT-B $\left(t_{(52)}=-3.53, p<.01\right)$ were considered. 
Table 12. Independent Samples t-Test comparing Dyadic Coping, Mutuality, Communication, and Quality of Life among younger and older breast cancer patients

\begin{tabular}{|c|c|c|c|c|c|c|c|}
\hline \multirow[b]{2}{*}{ Variable } & \multirow[b]{2}{*}{ Age Group } & \multirow[b]{2}{*}{$\mathbf{M}$} & \multirow[b]{2}{*}{ SD } & \multirow[b]{2}{*}{$t$} & \multirow[b]{2}{*}{$p$} & \multicolumn{2}{|c|}{$95 \% \mathrm{CI}$} \\
\hline & & & & & & $\mathbf{L L}$ & $\mathbf{U L}$ \\
\hline Stress & Younger Patients & 4.14 & .78900 & -.71 & .48 & -.43076 & .20197 \\
\hline Communication & Older Patients & 4.26 & .67764 & & & -.44122 & .21242 \\
\hline Common & Younger Patients & 3.53 & .67158 & -.77 & .44 & -.45235 & .19947 \\
\hline Dyadic Coping & Older Patients & 3.65 & .79367 & & & -.44257 & .18969 \\
\hline Positive & Younger Patients & 4.08 & .82055 & -.18 & .85 & -.38577 & . .32202 \\
\hline Dyadic Coping & Older Patients & 4.12 & .80406 & & & -.38798 & .32423 \\
\hline Hostile & Younger Patients & 2.07 & .57317 & 1.45 & .15 & -.06285 & .39823 \\
\hline Dyadic Coping & Older Patients & 1.90 & .49524 & & & -.07019 & .40557 \\
\hline Avoidance of & Younger Patients & 2.79 & .89302 & 0.94 & .35 & -.20135 & .56117 \\
\hline Dyadic Coping & Older Patients & 2.61 & .85990 & & & -.20507 & .56488 \\
\hline Total Dyadic & Younger Patients & 175.14 & 23.744 & 0.46 & .64 & -8.564 & 13.752 \\
\hline Coping & Older Patients & 172.55 & 26.728 & & & -8.330 & 13.518 \\
\hline Mutuality & Younger Patients & 4.38 & .70105 & -0.57 & .57 & -.37247 & .20653 \\
\hline & Older Patients & 4.46 & .63623 & & & -.37877 & .21283 \\
\hline Communication & Younger Patients & 38.34 & 7.01655 & 0.52 & .60 & -2.29085 & 3.91774 \\
\hline & Older Patients & 37.53 & 7.17594 & & & -2.28366 & 3.91055 \\
\hline Physical & Younger Patients & 18.51 & 6.25044 & -3.38 & $.001 * *$ & -6.19362 & -1.81667 \\
\hline Well-being & Older Patients & 22.52 & 4.05540 & & & -6.38232 & -1.62797 \\
\hline Social & Younger Patients & 21.51 & 4.06780 & -2.33 & $.022 *$ & -3.61431 & -.28760 \\
\hline Well-being & Older Patients & 23.47 & 3.62548 & & & -3.65668 & -.24522 \\
\hline Emotional & Younger Patients & 15.14 & 3.64726 & -3.26 & $.002 * *$ & -3.73119 & -.99486 \\
\hline Well-being & Older Patients & 17.50 & 2.73126 & & & -3.81461 & -.91144 \\
\hline Functional & Younger Patients & 17.60 & 5.65269 & -1.47 & .14 & -3.99774 & .59382 \\
\hline Well-being & Older Patients & 19.30 & 4.97455 & & & -4.06219 & .65826 \\
\hline Breast Cancer & Younger Patients & 22.59 & 5.69697 & -2.86 & $.005 * *$ & -5.21721 & -.94315 \\
\hline Symptoms & Older Patients & 25.67 & 4.26607 & & & -5.34753 & -.81284 \\
\hline FACT-G & Younger Patients & 72.77 & 14.50926 & -3.51 & $.001 * *$ & -15.37418 & -4.66798 \\
\hline & Older Patients & 82.79 & 10.46523 & & & -15.74074 & -4.30142 \\
\hline FACT-B & Younger Patients & 95.36 & 19.39514 & -3.53 & $.001 * *$ & -19.90846 & -6.29407 \\
\hline & Older Patients & 108.46 & 12.36090 & & & -20.53769 & -5.66483 \\
\hline
\end{tabular}




\section{Hypothesis 1.2: Younger partners will report lower quality of life, mutuality, communication and higher negative dyadic coping styles compared to older partners of breast cancer patients}

An independent-samples $t$ test was conducted to compare the mean scores of quality of life, mutuality, communication and dyadic coping styles of younger and older partners of breast cancer patients. Results indicate that although partners show similar scores on mutuality and communication, the younger group did report higher scores of maladaptive dyadic coping, lower mean scores of stress communication, common and positive dyadic coping, next to worse quality of life than the older group (Table 13). Significant mean differences between the two groups have been identified for hostile dyadic coping $\left(t_{(84)}=\right.$ $3.16, p<.01)$, emotional well-being $\left(t_{(84)}=-3.45, p<.01\right)$ and illness intrusivenesss $\left(t_{(84)}=\right.$ $3.75, p<.001)$. 
Table 13. Independent Samples t-Test comparing Dyadic Coping, Mutuality, Communication, and Quality of Life among younger and older partners

\begin{tabular}{|c|c|c|c|c|c|c|c|}
\hline \multirow[b]{2}{*}{ Variable } & \multirow[b]{2}{*}{ Age Group } & \multirow[b]{2}{*}{$\mathbf{M}$} & \multirow[b]{2}{*}{ SD } & \multirow[b]{2}{*}{$\mathbf{t}$} & \multirow[b]{2}{*}{$p$} & \multicolumn{2}{|c|}{$95 \% \mathrm{CI}$} \\
\hline & & & & & & $\mathbf{L L}$ & UL \\
\hline Stress & Younger Partners & 3.65 & .70 & -.75 & .45 & -.44552 & .20087 \\
\hline Communication & Older Partners & 3.49 & .77 & & & -.44050 & .19585 \\
\hline Common Dyadic & Younger Partners & 3.51 & .52 & -.93 & .33 & -.40439 & .14677 \\
\hline Coping & Older Partners & 3.63 & .69 & & & -.39016 & .13255 \\
\hline Positive & Younger Partners & 3.88 & .56 & -1.41 & .18 & -.49246 & .09644 \\
\hline Dyadic Coping & Older Partners & 4.08 & .74 & & & -.47787 & .08185 \\
\hline Hostile Dyadic & Younger Partners & 2.13 & .45 & 3.16 & $.002 * *$ & .11869 & .52308 \\
\hline Coping & Older Partners & 1.81 & .47 & & & .11956 & .52221 \\
\hline Avoidance of & Younger Partners & 2.69 & .75 & 1.14 & .256 & -.13194 & .48973 \\
\hline Dyadic Coping & Older Partners & 2.51 & .68 & & & -.13916 & .49696 \\
\hline Total Dyadic & Younger Partners & 163.68 & .48085 & -.84 & .40 & -12.94421 & 5.21760 \\
\hline Coping & Older Partners & 167.54 & .78093 & & & -12.33660 & 4.60999 \\
\hline \multirow[t]{2}{*}{ Mutuality } & Younger Partners & 4.45 & 0.48 & -.22 & .82 & -.33734 & .11825 \\
\hline & Older Partners & 4.48 & 0.78 & & & -.33204 & .11294 \\
\hline \multirow[t]{2}{*}{ Communication } & Younger Partners & 38.40 & 7.17 & -.37 & .71 & -3.65561 & 2.49482 \\
\hline & Older Partners & 38.98 & 6.95 & & & -3.68141 & 2.52063 \\
\hline Emotional & Younger Partners & 58.83 & 12.91 & -3.45 & $.001 * *$ & -15.19395 & -4.09008 \\
\hline Well-being & Older Partners & 68.47 & 12.58 & & & -15.23441 & -4.04963 \\
\hline Illness & Younger Partners & 43.06 & 14.12 & 3.75 & $<001 * * *$ & 5.33111 & 17.37142 \\
\hline Intrusiveness & Older Partners & 31.71 & 13.56 & & & 5.26941 & 17.43311 \\
\hline
\end{tabular}




\section{Hypothesis 1.3: The association of quality of life scores between patients and partners in older couples will be higher than the association of quality of life scores of patients and partners in younger couples}

After examing differences among mean scores of younger and older patients and partners, the association between quality of life and dyadic coping scores in the two groups was investigated. Pearson $r$ correlations were calculated to examine the level of association between quality of life scores among the two partners in younger and older couples (Table 14 and Table 15). Results indicate that the physical and social well-being of younger patients is associated to higher emotional well-being of the partner $(r=.34$, $p<.05 ; r=.37, p<.05)$. Similarly, moderate positive correlations are registered between total scores for quality of life and partner's well-being (FACT-G: $r=.43, p<.01$; FACTB: $r=.42, p<.05)$. When the analysis was conducted on the older group, significant associations were detected for both illness intrusiveness and emotional well-being of partners. In particular, higher scores on social well-being of patients were associated to reduced level of illness intrusiveness $(r=-.20, p<.05)$ and higher emotional well-being of the partner $(r=.32, p<.05)$. The same direction of association was registered for women's physical well-being and illness intrusiveness $(r=-.33, p<.05)$. Finally, as overall quality of life of older cancer patients improved, so the intrusiveness of the illness' demands on the caregiver was reduced $(r=-.30, p<.05 ; r=-.31, \mathrm{p}<.05)$ and emotional well-being improved $(r=.29, p<.05)$.

Contrary to our expectations, correlations coefficients of younger couples were higher. However, the association between patients and partners' quality of life in older couples exists for both measures of quality of life, while among the younger group significant correlations are limited to emotional well-being. 
Table 14. Bivariate Correlations of Quality of Life Scores of Younger Patients and Partners

\begin{tabular}{|c|c|c|c|c|c|c|c|c|c|}
\hline Variable & 1 & 2 & 3 & 4 & 5 & 6 & 7 & 8 & 9 \\
\hline 1. Physical Well-being Patient & & .17 & $.51 * *$ & $.55^{* *}$ & $.50^{* *}$ & $.76 * * *$ & $.75 * * *$ &.-.26 & $.34 *$ \\
\hline 2. Social Well-being Patient & & & .14 & $.33^{*}$ & $.45^{* *}$ & $.54 * *$ & $.56^{* *}$ & .05 & $.37 *$ \\
\hline 3. Emotional Well-being Patient & & & & $.58 * * *$ & $.38 *$ & $.73 * * *$ & $.68 * * *$ & -.01 & .19 \\
\hline 4. Functional Well-being Patient & & & & & $.43^{* *}$ & $.86 * * *$ & $.81 * * *$ &.-19 & .31 \\
\hline 5. Breast Cancer Symptoms Patient & & & & & & $.60 * * *$ & $.76 * * *$ &.-.01 & .32 \\
\hline 6. FACT-G Patient & & & & & & & $.97 * * *$ & -.18 & $.43 * *$ \\
\hline 7. FACT-B Patient & & & & & & & &.-13 & $.42 *$ \\
\hline 8. Illness Intrusiveness Partner & & & & & & & & & $-.44 * *$ \\
\hline 9. Emotional Well-being Partner & & & & & & & & & \\
\hline
\end{tabular}

Note: Illness Intrusiveness: higher scores indicate higher levels of intrusiveness; Emotional Well-being:: higher scores on this variable indicate better quality of life.

$\underset{\omega}{\omega} \quad$ Table 15. Bivariate Correlations of Quality of Life Scores of Older Patients and Partners

\begin{tabular}{|c|c|c|c|c|c|c|c|c|c|}
\hline Variable & 1 & 2 & 3 & 4 & 5 & 6 & 7 & 8 & 9 \\
\hline 1. Physical Well-being Patient & & .18 & .21 & $.60 * * *$ & $.62 * *$ & $.66 * * *$ & $.71 * * *$ & $-.33 *$ & .12 \\
\hline 2. Social Well-being Patient & & & .18 & $.39 * *$ & .16 & $.58 * * *$ & $.52 * * *$ & $-.20 *$ & $.32 *$ \\
\hline 3. Emotional Well-being Patient & & & & $.43 * *$ & $.34 *$ & $.57 * * *$ & $.56 * * *$ & -.17 & .08 \\
\hline 4. Functional Well-being Patient & & & & & $.50 * * *$ & $.88 * * *$ & $.86 * * *$ & -.19 & .25 \\
\hline 5. Breast Cancer Symptoms Patient & & & & & & $.54 * * *$ & $.72 * * *$ & -.18 & .17 \\
\hline 6. FACT-G Patient & & & & & & & $.97 * * *$ & $-.30 *$ & $.29 *$ \\
\hline 7. FACT-B Patient & & & & & & & & $-.31 *$ & .24 \\
\hline 8. Illness Intrusiveness Partner & & & & & & & & & $-.44 * *$ \\
\hline 9. Emotional Well-being Partner & & & & & & & & & \\
\hline
\end{tabular}

Note: Illness Intrusiveness: higher scores indicate higher levels of intrusiveness; Emotional Well-being:: higher scores on this variable indicate better quality of life. 


\section{Hypothesis 1.4: The association of dyadic coping scores between patients and partners in older couples will be higher than the association of dyadic coping scores of patients and partners in younger couples}

Pearson $r$ Product Coefficients were calculated to examine the level of association between dyadic coping scores of patients and partners in younger and older couples (Table 16 and Table 17). Results indicate that younger days are characterized by the presence of significant large correlations between common and positive dyadic coping scores of the two partners $(r=.57, p<.001$ and $r=.61, p<.001)$. A large negative correlation was also reported between common dyadic coping of the patient and hostile dyadic coping of the partner, indicating that as the woman's score on common dyadic coping increases, so hostile dyadic coping behaviors of the caregiver decrease $(r=-.52$, $p<.01)$. In the same direction, but at a moderate level, a negative association exists between common dyadic coping and avoidance of dyadic coping: the higher the level of common dyadic coping reported, the lower is the tendency of the partner to refrain from engaging in supportive coping behaviors. A similar situation is registered with positive dyadic coping: moderate positive correlations are identified between positive dyadic coping of the patient and the common and positive dyadic coping score of the caregiver $(r=.39, p<.05 ; r=.44, p<.01)$. In the expected direction are the negative correlations recorded for maladaptive coping styles: as the score of the woman increases on positive dyadic coping, so a reduction in hostile and avoidance of dyadic coping scores of the partners occurs $(r=-.36, p<.05 ; r=-.38, p<.05)$. It is important to note the absence of correlation between negative dyadic coping scores of the patient and any of the coping styles of the partner, because this represent a difference between younger and older dyads. 
If we focus our attention on the older group, we notice that moderate correlations exist between common dyadic coping of patients and common and positive dyadic coping of the partners $(r=.44, p<.01 ; r=.45, p<.01)$. In the opposite direction are the associations between common dyadic coping of the patients and the partners' scores on hostile and avoidance of dyadic coping; with reduction of the maladaptive forms of coping as the patients' score increases. Similar scores and the same direction of association are found when positive daydic coping is analyzed. Very interesting are the results obtained for avoidance of dyadic coping in the patient. Higher scores on this maladative coping style of older patients are associated with reduced common and dyadic coping for their spouses $(r=.30, p<.01 ; r=-.30, p<.05)$.

Three are the elements that emerge from this analysis. First, in both samples stress communication scores of the partners were not associated with any of the other coping styles. Second, if we compare the correlation coefficients, higher associations were registered especially between the common dyadic coping of younger couples. Third, in the older group patients and partners' association of dyadic coping scores extended also to negative coping styles, while this was not reported in the younger dyads. 
Table 16. Bivariate Correlations of Dyadic Coping Scores of Younger Patients and Partners

\begin{tabular}{|c|c|c|c|c|c|c|c|c|c|c|}
\hline Variable & 1 & 2 & 3 & 4 & 5 & 6 & 7 & 8 & 9 & 10 \\
\hline 1. Stress Communication Patient & & $.55^{* *}$ & $.66^{* * *}$ & $-.42 *$ & $-.38^{*}$ & -.22 & .20 & .21 & -.13 & -.15 \\
\hline 2.Common Dyadic Coping Patient & & & $.89 * * *$ & -.32 & $-.42 *$ & .02 & $.57 * * *$ & $.61 * * *$ & $-.52 * *$ & $-.46 * *$ \\
\hline 3.Positive Dyadic Coping Patient & & & & $-.60 * * *$ & $-.58 * * *$ & -.02 & $.38 *$ & $.44 * *$ & $-.36^{*}$ & $-.38 *$ \\
\hline 4. Hostile Dyadic Coping Patient & & & & & $.70 * *$ & .16 & .01 & .04 & .01 & .12 \\
\hline 5. Avoidance of Dyadic Coping Patient & & & & & . & .18 & .10 & .12 & .13 & .11 \\
\hline 6. Stress Communication Partner & & & & & & & $.41^{*}$ & $.34 *$ & -.21 & -.29 \\
\hline 7. Common Dyadic Coping Partner & & & & & & & & $.92 * *$ & $-.49 * *$ & $-.54 * *$ \\
\hline 8. Positive Dyadic Coping Partner & & & & & & & & & $-.58 * * *$ & $-.64 * * *$ \\
\hline 9. Hostile Dyadic Coping Partner & & & & & & & & & & $.65 * * *$ \\
\hline 10. Avoidance of Dyadic Coping Partner & & & & & & & & & & \\
\hline
\end{tabular}

Table 17. Bivariate Correlations of Dyadic Coping Scores of Older Patients and Partners

\begin{tabular}{|c|c|c|c|c|c|c|c|c|c|c|}
\hline Variable & 1 & 2 & 3 & 4 & 5 & 6 & 7 & 8 & 9 & 10 \\
\hline 1. Stress Communication Patient & & $.55 * * *$ & $.57 * * *$ & .14 & $-.33^{*}$ & .09 & .25 & .27 & .01 & -.08 \\
\hline 3.Positive Dyadic Coping Patient & & & & -.23 & $-.56^{* * *}$ & .01 & $.39 * *$ & $.39 * *$ & $-.38 * *$ & $-.34 *$ \\
\hline 5. Avoidance of Dyadic Coping Patient & & & & & & -.06 & $-.33 * *$ & $-.30 *$ & .27 & .27 \\
\hline 6. Stress Communication Partner & & & & & & & $.45^{* * *}$ & $.46^{* *}$ & .15 & -.07 \\
\hline 7. Common Dyadic Coping Partner & & & & & & & & $.93 * * *$ & $-.36 * *$ & $-.40 * *$ \\
\hline 9. Hostile Dyadic Coping Partner & & & & & & & & & & $.36^{* *}$ \\
\hline 10. Avoidance of Dyadic Coping Partner & & & & & & & & & & \\
\hline
\end{tabular}


Specific Aim 2: To examine the relationship between dyadic coping and quality of life for younger breast cancer patients and partners

Hypothesis 2.1: Positive forms of dyadic coping (Stress Communication, Common Dyadic Coping, Positive Dyadic Coping), will be associated with higher levels of quality of life for younger breast cancer patients

Since positive forms of dyadic coping have been consistently associated with higher quality of life, we were interested in testing this association among younger patients and partners. Findings from the Pearson $r$ coefficients (Table 18) revealed that stress communication is significantly associated to physical well-being $(r=-.38, p<.05)$, suggesting that as the physical well-being of the patient improves, so the level of stress communication decreases. Positive associations were found between social well-being and stress communication $(r=.45, p<.01)$, common dyadic coping $(r=.56, p<.001)$, and positive dyadic coping $(r=.58, p<.001)$. On the contrary, reduced social well-being was found for individuals reporting hostile dyadic coping $(r=-.48, p<.01)$ and avoidance of dyadic coping $(r=-.53, p<.01)$. Hostile dyadic coping, in particular, was also associated with worse level of overall quality of life (FACT-G: $r=-.38, p<.05$; FACT-B: $r=-.41$, $p<.051)$ and breast cancer symptoms, as measured by the subscale "additional concerns" $(r=-.41, p<.05)$.

It is therefore possible to affirm that, as hypothesized, positive forms of dyadic coping are associated with higher levels of quality of life in younger women with breast cancer and that this association has been found especially for their social well-being. 
Table 18. Bivariate Correlations for Younger Breast Cancer Patients Scores on Quality of Life and Dyadic Coping

\begin{tabular}{|c|c|c|c|c|c|c|c|c|c|c|c|c|}
\hline Variables & 1 & 2 & 3 & 4 & 5 & 6 & 7 & 8 & 9 & 10 & 11 & 12 \\
\hline 1. $\begin{array}{l}\text { Stress } \\
\text { Communication }\end{array}$ & & $.48^{* *}$ & $.60 * *$ & -.24 & $-.39 *$ & $-.37 *$ & $.45 * *$ & -.30 & -.18 & -.11 & -.23 & -.21 \\
\hline $\begin{array}{l}\text { 2. Common } \\
\text { Dyadic Coping }\end{array}$ & & & $.86^{* * *}$ & $-.59 * * *$ & $-.63 * * *$ & .02 & $.56^{* * * *}$ & -.18 & -.03 & .15 & .09 . & .11 \\
\hline $\begin{array}{l}\text { 3. Positive } \\
\text { Dyadic Coping }\end{array}$ & & & & $-.63 * * *$ & $-.66^{* * *}$ & -.19 & $.58 * * *$ & -.23 & -.06 & .04 & .02 & .02 \\
\hline $\begin{array}{l}\text { 4. Hostile } \\
\text { Dyadic Coping }\end{array}$ & & & & & $.62 * * *$ & -.24 & $-.48 * *$ & -.04 & -.28 & $-.41 *$ & $-.38^{*}$ & $-.41 *$ \\
\hline $\begin{array}{l}\text { 5. Avoidance of } \\
\text { Dyadic Coping }\end{array}$ & & & & & & .07 & $-.53 * *$ & .11 & -.16 & -.08 & -.13 & -.12 \\
\hline $\begin{array}{l}\text { 6. Physical } \\
\text { Well-being }\end{array}$ & & . & . & & & & .24 & .23 & $.72 * * *$ & $.64 * * *$ & $.79 * * *$ & $.78 * * *$ \\
\hline $\begin{array}{l}\text { 7. Social } \\
\text { Well-being }\end{array}$ & & & . & & & & & -.03 & $.42 *$ & $.48 * *$ & $.50 * *$ & $.51 * *$ \\
\hline $\begin{array}{l}\text { 8. Emotional } \\
\text { Well-being }\end{array}$ & & . & . & & & & & & $.47 * *$ & $.45^{* *}$ & $.51 * *$ & $.51 * *$ \\
\hline $\begin{array}{l}\text { 9. Functional } \\
\text { Well-being }\end{array}$ & & & & & & & & & & $.73 * * *$ & $.92 * * *$ & $.90 * * *$ \\
\hline $\begin{array}{l}\text { 10. Breast Cancer } \\
\text { Symptoms }\end{array}$ & & & & & & & & & & & $.81 * * *$ & $.89 * * *$ \\
\hline $\begin{array}{l}\text { 11. FACT-G } \\
\text { 12. FACT-B }\end{array}$ & & & & & & & & & & & & $.98 * * *$ \\
\hline
\end{tabular}


Hypothesis 2.2: Positive forms of dyadic coping (Stress Communication, Common Dyadic Coping, Positive Dyadic Coping), will be associated to higher levels of quality of life for partners of younger breast cancer patients

Pearson $r$ Product Coefficients have been calculated to examine the relationship between dyadic coping and quality of life of partners of younger women with breast cancer (Table 19). In contrast to the hypothesis, results from the bivariate analysis revealed the absence of a significant association between all of the categories of dyadic coping and quality of life scores for younger partners. 
Table 19. Bivariate Correlations for Younger Partners Scores on Quality of Life and Dyadic Coping

\begin{tabular}{|c|c|c|c|c|c|c|c|c|}
\hline Variables & 1 & 2 & 3 & 4 & 5 & 6 & 7 & 8 \\
\hline 1. Stress Communication & & $.41 * *$ & $.34 * *$ & -.21 & -.29 & $.52 * *$ & -.21 & .17 \\
\hline $\begin{array}{l}\text { 2. Common } \\
\text { Dyadic Coping }\end{array}$ & & & $.92 * * *$ & $-.49 * * *$ & $-.54 * * *$ & $.89 * * *$ & -.07 & .19 \\
\hline $\begin{array}{l}\text { 3. Positive } \\
\text { Dyadic Coping }\end{array}$ & & & & $-.58 * * *$ & $-.64 * * *$ & $.93 * * *$ & .06 & .13 \\
\hline $\begin{array}{l}\text { 4. Hostile } \\
\text { Dyadic Coping }\end{array}$ & & & & & $.65 * * *$ & $-.334 *$ & -.28 & .11 \\
\hline $\begin{array}{l}\text { 5. Avoidance } \\
\text { of Dyadic Coping }\end{array}$ & & & & & & $-.46 * *$ & -.30 & -.15 \\
\hline 6. Total Dyadic Coping & & & & & & & -.10 & .19 \\
\hline $\begin{array}{l}\text { 7. Emotional Well-being } \\
\text { 8. Illness Intrusiveness }\end{array}$ & & & & & & & & -.31 \\
\hline
\end{tabular}




\section{Hypothesis 2.3: The relationship between dyadic coping styles and quality of life for patients and partners is influenced by socio-demographic, clinical, relational and mental health variables}

In order to determine if the relationship between dyadic coping and quality of life differed by socio-demographic, clinical, relational and mental health variables, Pearson $r$ correlation coefficients ${ }^{26}$ were calculated. Using these results to select significant predictors of quality of life, a series of multiple regression analysis were run to test the the influence of dyadic coping along with the control variables on women's and partners' quality of life. As the goal of the present study was to understand differences in the developmental trajectory of dyadic coping over the life-span, age was entered in each multiple regression ${ }^{27}$ and the whole sample of patients was included in the analysis. This

\footnotetext{
${ }^{26}$ Findings from the bivariate analysis revealed several significant relationships. For patients Hostile Dyadic Coping was associated with the current use of medication $(r=.25, \mathrm{p}<.05)$ while Common Dyadic Coping increased for women who haven't been diagnosed with depression in the past $(r=.-.23, p<.05)$. Among socio-economic factors, age was correlated with several subscales of the FACT-B. Positive associations were revealed between age and Physical Well-being $(\mathrm{r}=.27, \mathrm{p}<.05, \mathrm{r}=.32, \mathrm{p}<.01)$, Emotional Well-being and the two total scores of the FACT-G $(r=.29, \mathrm{p}<.01)$ and FACT-B $(r=.28, \mathrm{p}<.05)$. Additionally, higher levels of quality of life were associated to length of relationship. Moderate positive correlation coefficients were recorded for Physical Well-being $(r=.25, p<.05)$, Social Well-being $(r=.21$, $\mathrm{p}<.05)$, Emotional Well-being $(\mathrm{r}=.28, \mathrm{p}<.05)$, FACT-G $(\mathrm{r}=.31, \mathrm{p}<.01)$ and the total score on the FACT-B $(\mathrm{r}=.28, \mathrm{p}<.01)$, suggesting that breast cancer patients in more stable and longer relationship reported higher quality of life scores on all of these subscales.

Findings also show that receiving chemotherapy and medications as part of the cancer treatment was negatively associated to numerous aspect of the individual's quality of life, with negative correlations registered for Physical well-being $(r=-.33, p<.01)$, Functional Well-being $(r=.24, p<.05)$, Breast Cancer Symptoms $(\mathrm{r}=.36, \mathrm{p}<.01)$ and the overall FACT-G $(\mathrm{r}=-.26, \mathrm{p}<.05)$ and FACT-B scores $(\mathrm{r}=-.31, \mathrm{p}<.01)$. Chemotherapy was linked to reduced Physical $(\mathrm{r}=-.33, \mathrm{p}<.01)$, Functional Well-being $(\mathrm{r}=-.22, \mathrm{p}<.05)$, higher impact of cancer symptoms $(r=.-32, p<.01)$ and overall worse scores on the total FACT-G $(r=-.26$, $\mathrm{p}<.05)$ and FACT-B $(r=-.30, \mathrm{p}<.01)$. For women with breast cancer having received a previous diagnosis of depression was associated to negative Functional Well-being $(r=-.27, p<.05)$ and worse overall functioning on the FACT-G subscale $(\mathrm{r}=-.22, \mathrm{p}<.05)$. With regard to the impact of dyadic coping on the woman's quality of life, numerous associations have been detected. Adaptive dyadic coping styles contributed to higher Social Well-being, as indicated by positive moderate correlations between Social Well-being and Stress Communication $(r=.32, \mathrm{p}<.01)$, Common Dyadic Coping $(\mathrm{r}=.26, \mathrm{p}<.05)$ and Positive Dyadic Coping $(r=.30, p<.01)$. On the contrary, maladaptive coping styles like Hostile and Avoidance of Dyadic Coping were associated to reduced scores on this subscale $(r=-.35, p<.01$ and $r=-$ $.27, \mathrm{p}<.05)$. A negative correlation was registered between Total Dyadic Coping and Emotional Well-being $(\mathrm{r}=-. .21, \mathrm{p}<.05)$. The negative association of Hostile Dyadic Coping and the quality of life of women with breast cancer was confirmed by negative correlations identified with Breast Cancer Symptoms $(r=-.32$, $\mathrm{p}<.05)$, FACT-G $(\mathrm{r}=--.22, \mathrm{p}<.05)$ and the total score on the FACT-B $(\mathrm{r}=-.27, \mathrm{p}<.05)$.

${ }^{27}$ Another option was to conduct the analysis including a dummy variable in the equation. It was however preferred to treat age as continuous.
} 
strategy was selected to meet the sample size indication from Tabachnick and Fidell (2007), as the younger group of patients is limited to only 35 individuals ${ }^{28}$. All regression equations used a backward method of model building: all predictors were entered in the equation and then non-significant variables were removed one at a time ${ }^{29}$. Removal stopped when $\mathrm{R}$ squared values decreased and indication of problematic correlation among predictors were identified.

The first equation regressed the outcome variable social well-being on age, length of relationship, stress communication, positive dyadic coping, hostile dyadic coping and avoidance dyadic coping. Because of multicollinearity, common dyadic coping was removed from the analysis ${ }^{30}$. Although it is recommended to not include in the same analysis subscales of an instrument (Meyers et al., 2006), in this case stress communication, positive, hostile and avoidance of dyadic coping were retained because of their correlation coefficients (which were below the problematic correlation of .75 as suggested by Meyers et al., 2006) and because of the absence of multicollinearity issues as evidenced by Tolerance and VIF (Variance Inflation Factor) scores in the multiple regression analysis.

\footnotetext{
${ }^{28}$ Required sample size is influenced by desired power, alpha level, number of predictors and expected effect sizes. A general indication provided by Tabachnick and Fidell (2007) is to conduct regression analysis when $\mathrm{N} \geq 50+8 m$ (where $m$ is the number of IVs), assuming a medium-size relationship between IVs and the DV, .05 and $\beta=.20$. Since only 35 patients and 35 partners were considered young, running the analysis only on them would have been incorrect. It was then decided to use as sample the 86 women and 86 partners included in the study.

${ }^{29}$ This strategy was preferred to the simultaneous and stepwise method because of the limited sample size and the absence of a theoretical justification for the entry order of variables. A first attempt was made with the simultaneous/enter option but led to non-significant findings, which improved with following removal of variables.

${ }^{30}$ A very large positive correlation exists between Positive and Common Dyadic Coping $(r=.90, p<.001)$. On the contrary Age and Length of Relationship were kept in the analysis because Collinearity Statistics, and in particular the Variance Inflation Factor, did not indicate the existence of problematic collinearity.
} 


$$
\hat{Y}_{s w b}=\mathrm{a}+\mathrm{b}_{1}(\text { Age })+\mathrm{b}_{2}(\text { Length of Relationship })+\mathrm{b}_{3}(\text { Stress Communication })+\mathrm{b}_{4}
$$

(Positive Dyadic Coping) $+\mathrm{b}_{5}($ Hostile Dyadic Coping $)+\mathrm{b}_{6}$ (Avoidance of Dyadic

$$
\text { Coping) }+e
$$

The second equation regressed the outcome variable of emotional well-being on age, length of relationship and total dyadic coping.

$$
\widehat{Y}_{e w \mathrm{~b}}=\mathrm{a}+\mathrm{b}_{1}(\text { Age })+\mathrm{b}_{2}(\text { Length of Relationship })+\mathrm{b}_{3}(\text { Total Dyadic Coping })+e
$$

The third equation regressed the outcome variable FACT-G on age, length of relationship, chemotherapy, medications and hostile dyadic coping.

$$
\begin{gathered}
\hat{Y}_{\text {Fact }-G}=\mathrm{a}+\mathrm{b}_{1}(\text { Age })+\mathrm{b}_{2}(\text { Length of Relationship })+\mathrm{b}_{3}(\text { Chemotherapy })+\mathrm{b}_{4} \\
\text { (Medications })+\mathrm{b}_{5}(\text { Hostile Dyadic Coping })+e
\end{gathered}
$$

The fourth equation regressed the outcome breast cancer symptoms on age, medications, chemotherapy and hostile dyadic coping.

$$
\begin{gathered}
\left.\hat{Y}_{\mathrm{bcs}}=\mathrm{a}+\mathrm{b}_{1}(\text { Age })+\mathrm{b}_{2} \text { (Medications }\right)+\mathrm{b}_{3}(\text { Chemotherapy })+\mathrm{b}_{4} \text { (Hostile Dyadic } \\
\text { Coping })+e
\end{gathered}
$$

The fifth equation regressed the outcome variable FACT-B on age, length of relationship, medications, chemotherapy and hostile dyadic coping.

$$
\begin{aligned}
& \hat{Y}_{\text {fact-b }}=\mathrm{a}+\mathrm{b}_{1}(\text { Age })+\mathrm{b}_{2}(\text { Length of Relationship })+\mathrm{b}_{3}(\text { Medication })+\mathrm{b}_{4} \\
& \text { (Chemotherapy) }+\mathrm{b}_{5}(\text { Hostile Dyadic Coping })+e
\end{aligned}
$$

Physical and functional well-being were excluded from present analyses due to the absence of significant correlation with any dyadic coping style. Before conducting the 
analyses described, Skewness and Kurtosis were examined again for all the variables included. Length of relationship presented moderate kurtosis (-.1.17), use of medications was characterized by substantial negative kurtosis (-1.82), chemotherapy was moderately skewed (1.42) as well as treatment for depression (1.14). Numerous transformations have been attempted but none could improve the distribution of these variables, which were then retained in the analysis in their original presentation.

A multiple regression analysis was performed to investigate whether age, length of relationship, stress communication, positive dyadic coping, hostile dyadic coping and avoidance of dyadic coping significantly predicted social well-being scores of breast cancer patients (Table 20). The final model presents a $R^{2}$ statistic statistically significant, $\left(F(4,81)=6.54, p<.001 ; \mathrm{R}^{2}\right.$ Adjusted=.207), indicating that approximately $21 \%$ of variance in social well-being can be explained by the predictors. Length of relationship, stress communication and hostile dyadic coping significantly contributed to the prediction of social functioning performance. As hypothesized, higher well-being was predicted for women who have been involved in a relationship for a longer number of years and were able to communicate their needs to the partner, while the opposite was identified for women with breast cancer who reported higher scores on hostile dyadic coping, controlling for other variables. 
Table 20. Multiple Regression Analysis Predicting Social Well-being Score for Breast Cancer Patients

\begin{tabular}{|c|c|c|c|c|c|}
\hline \multirow[b]{2}{*}{ Variables } & \multicolumn{4}{|c|}{ Social Well-being Score } & \multirow[b]{2}{*}{$\begin{array}{c}\text { Part } \\
\text { Correlation }\end{array}$} \\
\hline & $\mathbf{B}$ & $\begin{array}{c}\text { Std. } \\
\text { Error(B) }\end{array}$ & $\boldsymbol{\beta}$ & $t$ & \\
\hline Constant & 20.28 & 3.39 & & $5.98 * * *$ & \\
\hline $\begin{array}{l}\text { Length of } \\
\text { Relationship } \\
\text { Stress }\end{array}$ & .06 & .03 & .19 & $2.02 *$ & .19 \\
\hline $\begin{array}{l}\text { Communication } \\
\text { Hostile Dyadic }\end{array}$ & 1.42 & .56 & .26 & $2.54 *$ & .24 \\
\hline $\begin{array}{l}\text { Coping } \\
\text { Avoidance of }\end{array}$ & -2.01 & .76 & -.27 & $-2.66 * *$ & -.26 \\
\hline Dyadic Coping & -0.33 & .49 & -.07 & -.67 & -.06 \\
\hline
\end{tabular}

Overall $\mathrm{R}^{2}=.244$, Adjusted $\mathrm{R}^{2}=.207, F(4,81)=6.54, \mathrm{p}<.001$

$* \mathrm{p}<.05 * * \mathrm{p}<.01 * * * \mathrm{p}<.001$

In a second analysis, age, length of relationship and total dyadic coping were entered in a multiple regression analysis to predict patients' emotional well-being (Table 21). Results show that age is a significant predictor of emotional well-being, with higher scores reported as age of patients increases. A one standard deviation increase in age (9.82 points) results in a .29 (Standardized Beta Weight) * 3.32 (SD of Emotional Wellbeing $)=.963$ points increase in emotional well-being. The final model presents a significant $\mathrm{R}^{2}$ statistic $\left(\mathrm{F}_{(2,83)}=6.10, p<.01, \mathrm{R}^{2}\right.$ Adjusted $\left.=.107\right)$, indicating that approximately $11 \%$ of variance in emotional well-being can be explained by the predictors. 
Table 21. Multiple Regression Analysis Predicting Emotional Well-being Score for Breast Cancer Patients

\begin{tabular}{lccccc}
\hline \multirow{2}{*}{ Variables } & $\mathbf{B}$ & $\begin{array}{c}\text { Std. } \\
\text { Error(B) }\end{array}$ & $\boldsymbol{\beta}$ & $\boldsymbol{t}$ & $\begin{array}{c}\text { Part } \\
\text { Correlation }\end{array}$ \\
\cline { 2 - 6 } & & 3.18 & $4.93 * * *$ \\
Constant & 15.70 & $\mathbf{. 0 3}$ & $\mathbf{. 2 9}$ & $\mathbf{2 . 8 0} * *$ & $\mathbf{. 2 9}$ \\
Age & $\mathbf{. 0 9}$ & .02 & -.17 & -1.61 & -.16 \\
Total Dyadic & -0.02 & & & \\
Coping & & & & \\
\hline Overall $\mathrm{R}^{2}=.128$, Adjusted $\mathrm{R}^{2}=.107, F(2,83)=6.10, \mathrm{p}<.01$ \\
$* \mathrm{p}<.05 * * \mathrm{p}<.01 * * * \mathrm{p}<.001$
\end{tabular}

Then, a multiple regression analysis was performed to investigate if an increase in patient's age, length of relationship, hostile dyadic coping and current treatment for breast cancer (medications and chemotherapy) contributed to the patients' score on the FACT-G subscale (Table 22). In the final model the $\mathrm{R}^{2}$ statistic was statistically significant $\left(F_{(3,80)}=5.82, p<.001\right.$ Adjusted $\left.\mathrm{R}^{2}=.188\right)$, indicating that approximately $19 \%$ of variance in in the quality of life of women with breast cancer can be explained by the present model. Age and currently taking medications were identified as significant predictors of the subscale score. Quality of life increases with age and for those not receiving additional medical treatment. Interestingly, hostile dyadic coping only approached significance $(p=.08)$. 
Table 22. Multiple Regression Analysis Predicting FACT-G Score for Breast Cancer Patients

\begin{tabular}{|c|c|c|c|c|c|}
\hline \multirow[b]{2}{*}{ Variables } & \multicolumn{4}{|c|}{ FACT-G Score } & \multirow[b]{2}{*}{$\begin{array}{c}\text { Part } \\
\text { Correlation } \\
\end{array}$} \\
\hline & B & $\begin{array}{c}\text { Std. } \\
\text { Error(B) }\end{array}$ & $\boldsymbol{\beta}$ & $t$ & \\
\hline Constant & 73.10 & 8.19 & & $8.92 * * *$ & \\
\hline Age & .38 & .13 & .29 & $2.88 * *$ & .28 \\
\hline Chemotherapy & -4.04 & 3.41 & -.13 & -1.19 & -.12 \\
\hline Medications & -6.02 & 2.93 & -.23 & $-2.05^{*}$ & -.20 \\
\hline Hostile & -4.43 & 2.52 & -.18 & -1.76 & -.17 \\
\hline \multicolumn{6}{|l|}{ Dyadic } \\
\hline \multicolumn{6}{|l|}{ Coping } \\
\hline \multicolumn{6}{|c|}{ Overall $\mathrm{R}^{2}=.228$, Adjusted $\mathrm{R}^{2}=.188, F(4,79)=5.82, \mathrm{p}<.001$} \\
\hline \multicolumn{6}{|c|}{ To predict the impairment in quality of life caused by the symptoms and limitations } \\
\hline \multicolumn{6}{|c|}{ of breast cancer, a multiple regression analysis was conducted (Table 23). Results } \\
\hline \multicolumn{6}{|c|}{ indicate that the final model was statistically significant $\left(F_{(4,81)}=6.54, p<.001\right.$. } \\
\hline \multicolumn{6}{|c|}{ Adjusted $\mathrm{R}^{2}=.227$ ), and that approximately $23 \%$ of the variance in breast cancer } \\
\hline \multicolumn{6}{|c|}{ symptoms was explained. In particular, hostile dyadic coping was a significant predictor } \\
\hline \multicolumn{6}{|c|}{ of higher impairment ${ }^{31}$, with the current use of medications being another significant } \\
\hline
\end{tabular}

${ }^{31}$ Higher scores on the subscales and on the two total scores of the FACT-B indicate higher quality of life. 
Table 23. Multiple Regression Analysis Predicting Breast Cancer Symptoms Score for Breast Cancer Patients

\begin{tabular}{|c|c|c|c|c|c|}
\hline \multirow[b]{2}{*}{ Variables } & \multicolumn{4}{|c|}{$\begin{array}{l}\text { Breast Cancer Symptoms } \\
\text { Score }\end{array}$} & \multirow[b]{2}{*}{$\begin{array}{c}\text { Part } \\
\text { Correlation }\end{array}$} \\
\hline & B & $\begin{array}{c}\text { Std. } \\
\text { Error(B) }\end{array}$ & $\boldsymbol{\beta}$ & $t$ & \\
\hline Constant & 26.95 & 3.09 & & $8.71 * * *$ & \\
\hline Age & .08 & .05 & .16 & 1.63 & .16 \\
\hline Medications & -2.79 & 1.11 & -.27 & $-2.52 *$ & -.24 \\
\hline \multicolumn{6}{|l|}{ Chemotherapy } \\
\hline & -2.12 & 1.29 & -.17 & -1.65 & -.16 \\
\hline Hostile & -2.28 & .95 & -.24 & $-2.40 *$ & -.23 \\
\hline \multicolumn{6}{|l|}{ Dyadic } \\
\hline \multicolumn{6}{|l|}{ Coping } \\
\hline \multicolumn{6}{|c|}{$\begin{array}{l}\text { Overall } \mathrm{R}^{2}=.264, \text { Adjusted } \mathrm{R}^{2}=.227, F(4,81)=6.54, \mathrm{p}<.001 \\
* \mathrm{p}<.05 * * \mathrm{p}<.01 * * * \mathrm{p}<.001\end{array}$} \\
\hline \multicolumn{6}{|c|}{ Finally, a multiple regression analysis examined the relationship between hostile } \\
\hline \multicolumn{6}{|c|}{ dyadic coping and the overall quality of life scores at the FACT-B, controlling for age, } \\
\hline \multicolumn{6}{|c|}{ length of relationship, medications, and chemotherapy (Table 24). The final model was } \\
\hline \multicolumn{6}{|c|}{ statistically significant $\left(F_{(4,79)}=7.47, p<.001\right.$. Adjusted $\left.\mathrm{R}^{2}=.238\right)$ and explained } \\
\hline \multicolumn{6}{|c|}{ approximately $24 \%$ of the variance in the total quality of life score. In particular, the } \\
\hline \multicolumn{6}{|c|}{ multiple regression analysis revealed that age, medications and hostile dyadic coping are } \\
\hline \multicolumn{6}{|c|}{ significant predictors of the FACT-B score, with better outcomes experienced as age of } \\
\hline \multicolumn{6}{|c|}{ the patient increases, by participants who were not taking medications at the time of the } \\
\hline
\end{tabular}


Table 24. Multiple Regression Analysis Predicting FACT-B Score of Breast Cancer

Patients

\begin{tabular}{lccccc}
\hline \multirow{2}{*}{ Variables } & \multicolumn{5}{c}{ FACT-B Score } \\
\cline { 2 - 6 } & $\mathbf{B}$ & $\begin{array}{c}\text { Std. } \\
\text { Error(B) }\end{array}$ & $\boldsymbol{\beta}$ & $\boldsymbol{t}$ & $\begin{array}{c}\text { Part } \\
\text { Correlation }\end{array}$ \\
\hline Constant & 100.54 & 10.83 & $9.88^{* * *}$ & \\
Age & $\mathbf{. 7 7}$ & $\mathbf{. 1 7}$ & $\mathbf{. 2 8}$ & $\mathbf{2 . 8 3} * *$ & $\mathbf{2 7}$ \\
Medications & $\mathbf{- 8 . 0 8}$ & $\mathbf{3 . 6 2}$ & $\mathbf{- . 2 6}$ & $\mathbf{- 2 . 4 3 *}$ & $\mathbf{- . 2 3}$ \\
Chemotherapy & -6.16 & 4.21 & -.15 & -1.60 & -.14 \\
Hostile & $\mathbf{- 6 . 7 1}$ & $\mathbf{3 . 6 2}$ & $\mathbf{- . 2 1}$ & $\mathbf{- 2 . 1 6 *}$ & $\mathbf{- . 2 1}$ \\
Dyadic & & & & \\
Coping & & & & \\
\hline Overall $\mathrm{R}^{2}=.275$, Adjusted $\mathrm{R}^{2}=.238, F(4,79)=7.47, \mathrm{p}<.001$ & \\
$* \mathrm{p}<.05 * * \mathrm{p}<.01 * * * \mathrm{p}<.001$ &
\end{tabular}

Similarly, correlations were run for dyadic coping styles, quality of life measures and socio-demographic, relational, and mental health variables for partners using Pearson $r$ correlation coefficients ${ }^{32}$. A series of multiple regressions were then conducted to test the the influence of dyadic coping along with the control variables on partners' quality of life. Like before for women, age was entered in each multiple regression.

The first equation regressed the outcome variables emotional well-being on age, length of relationship, hostile dyadic coping and avoidance of dyadic coping.

$$
\begin{gathered}
\left.\hat{Y}_{\text {ewb }}=\mathrm{a}+\mathrm{b}_{1}(\text { Age })+\mathrm{b}_{2} \text { (Length of Relationship }\right)+\mathrm{b}_{3}(\text { Hostile Dyadic Coping })+ \\
\mathrm{b}_{4}(\text { Avoidance of Dyadic Coping })+e
\end{gathered}
$$

\footnotetext{
${ }^{32}$ Results indicate the presence of a significant positive association between Emotional Well-being and Age $(\mathrm{r}=.34, \mathrm{p}<.01)$, suggesting that in the present sample quality of life increases for older caregivers.

Similarly, greater Emotional Well-being was recorded for individuals who have been in a relationship for a longer number of years $(r=.26, \mathrm{p}<.05)$. In the present study, lower emotional well-being characterized individuals who reported negative coping style, like Hostile Dyadic Coping $(r=-.31, \mathrm{p}<.01)$ and Avoidance of Dyadic Coping $(r=-.21, p<.05)$. Higher intrusiveness of cancer was identified for caregivers who were in less stable relationships $(\mathrm{r}=-.24, \mathrm{p}<.05)$ and for those who held more demanding occupations in terms of time and responsibilities $(r=.24, \mathrm{p}<.05)$. Finally, higher intrusiveness was registered for partners presenting maladaptive coping style, like Hostile Dyadic Coping $(r=.37, \mathrm{p}<.001)$.
} 
The $\mathrm{R}^{2}$ statistic was statistically significant $\left(\mathrm{F}_{(3,82)}=5.99 p<.01, \mathrm{R}^{2}\right.$ Adjusted=.150), indicating that $15 \%$ of variance in emotional well-being of partners is explained by the current analysis (Table 25). In the final model only age significantly contributed to the prediction of emotional well-being for partners, suggesting that well-being improves as age of the partners increases. On the contrary non-significant was the contribution of hostile and avoidance of dyadic coping.

The second equation regressed the outcome variable of illness intrusiveness on age, length of relationship, occupation and hostile dyadic coping.

$$
\begin{gathered}
\hat{Y}_{\text {iint } r}=\mathrm{a}+\mathrm{b}_{1}(\text { Age })+\mathrm{b}_{2}(\text { Length of Relationship })+\mathrm{b}_{3}(\text { Occupation })+\mathrm{b}_{4}(\text { Hostile } \\
\text { Dyadic Coping })+e
\end{gathered}
$$

The $\mathrm{R}^{2}$ statistic was statistically significant $\left(F_{(4,81)}=8.29, \mathrm{p}<.001\right.$; Adjusted $\mathrm{R}^{2=}$ .255 ) and the model accounted for approximately $25.5 \%$ of variance in illness intrusiveness (Table 26). Results of the present multiple regression analysis indicate that higher illness intrusiveness was reported by partners as age decreases and by those who had higher self-reported scores of maladaptive dyadic coping (hostile dyadic coping). 
Table 25. Multiple Regression Analysis Predicting Emotional Well-being of Partners

\begin{tabular}{|c|c|c|c|c|c|}
\hline \multirow[b]{2}{*}{ Variables } & \multicolumn{4}{|c|}{ Emotional Well-being Score } & \multirow[b]{2}{*}{$\begin{array}{c}\text { Part } \\
\text { Correlation }\end{array}$} \\
\hline & $\mathbf{B}$ & $\begin{array}{c}\text { Std. } \\
\text { Error(B) }\end{array}$ & Beta & $t$ & \\
\hline Constant & 61.43 & 9.86 & & $6.23 * * *$ & \\
\hline $\begin{array}{l}\text { Age } \\
\text { Hostile }\end{array}$ & .36 & .13 & .29 & $2.79 * *$ & .28 \\
\hline $\begin{array}{l}\text { Dyadic } \\
\text { Coping }\end{array}$ & -5.38 & 3.28 & -.19 & -1.64 & -.16 \\
\hline $\begin{array}{l}\text { Avoidance of } \\
\text { Dyadic } \\
\text { Coping }\end{array}$ & -1.82 & 2.18 & -.09 & -.83 & -.08 \\
\hline
\end{tabular}

Table 26. Multiple Regression Analysis Predicting Illness Intrusiveness of Partners

\begin{tabular}{lccccc}
\hline \multirow{2}{*}{ Variables } & $\mathbf{B}$ & $\begin{array}{c}\text { Std. } \\
\text { Error(B) }\end{array}$ & Beta & $t$ & $\begin{array}{c}\text { Part } \\
\text { Correlation }\end{array}$ \\
\cline { 2 - 6 } & & 11.02 & $3.15^{* * * *}$ & \\
Constant & 34.67 & $\mathbf{. 1 6}$ & $\mathbf{- . 3 9}$ & $\mathbf{- 3 . 0 7 * *}$ & $\mathbf{- 2 9}$ \\
Age & $\mathbf{- . 4 9}$ & .14 & .07 & .58 & .05 \\
$\begin{array}{l}\text { Length of } \\
\text { Relationship }\end{array}$ & .07 & 1.00 & .19 & 1.97 & .18 \\
$\begin{array}{l}\text { Occupation } \\
\text { Hostile }\end{array}$ & 1.97 & $\mathbf{3 . 0 2}$ & $\mathbf{. 2 9}$ & $\mathbf{2 . 9 6 * *}$ & $\mathbf{. 2 8}$ \\
$\begin{array}{l}\text { Dyadic } \\
\text { Coping }\end{array}$ & $\mathbf{8 . 7 9}$ & & & & \\
\hline
\end{tabular}

Overall $\mathrm{R}^{2}=.291$, Adjusted $\mathrm{R}^{2}=.255, F(4,81)=8.29, \mathrm{p}<.001$

$* \mathrm{p}<.05 * * \mathrm{p}<.01 * * * \mathrm{p}<.001$ 
Specific Aim 3: To assess the actor and partner effects of Mutuality on Dyadic Coping among breast cancer patients and partners and to identify differences by age group

Hypothesis 3.1: Patients' perceived mutuality will influence their own dyadic coping style and their partners' dyadic coping style

Hypothesis 3.2: Partners' perceived mutuality will influence their own dyadic coping style and the patients' dyadic coping style

Hypothesis 3.3: Differences in actor and partner effects of mutuality on dyadic coping exist by age group, between younger and older dyads

The third aim of the study was to assess the actor and partner effects of mutuality on dyadic coping among breast cancer patients and partners. In particular, it was hypothesized that patients' and partners' perceived mutuality will influence their own dyadic coping style, their partners' dyadic coping style, and that differences existed by age group. The Actor-Partner Interdependence Model (APIM) was used to examine whether this individual characteristic has interpersonal consequences on the dyadic coping style reported by each member of the dyad, controlling for the level of mutuality of each partner (Kashy \& Donnellan, 2012; Kashy \& Snyder, 1995; Kenny et al., 2006; Kenny, 1996).

To facilitate a clear understanding of the analysis and avoid confusion with the partner effect, partners will be addressed with the term "caregivers" in the present paragraph. Results are reported in tables and graphs, with a first table presenting multilevel regression coefficients estimating actor and partner effects. A second table includes the simple slopes regression coefficients estimating separate mutuality actor and partner effects for patients and caregivers on the different dyadic coping styles. Finally, the APIM model is traditionally summarized in the form of a figure where the actor and partner effects are visually displayed by the use of intersecting arrows. For the present 
analysis, standardized coefficients are presented for both actor and partner effects. If no significant interaction was found between role and actor or partner effect, then no difference exists between the actor and partner effects of patients and caregivers, and the standardized coefficient of the average effect is reported in the figure to remind the reader of its significance.

Two analyses were conducted: first a standard APIM model for distinguishable dyads was calculated with actor and partner mutuality scores used as predictors of dyadic coping for patients and caregivers. Second, to examine actor and partner effects in the two age groups, two separate APIM models were calculated for younger and older couples. This decision was supported by the significant differences identified between the two groups by the earlier analyses about the experience of coping with breast cancer. In the study of couples' coping and adjustment to stress, length of relationship is an important variable to control for, as it is well established that better relational outcomes are reported for couples who have been together longer. However, in the present analysis, it was decided not to add this variable to the equation for two reasons: length of relationship and age are highly correlated $(\mathrm{r}=.66, p<.001)$ in the present sample. Second, while a power analysis algorithm hasn't been developed yet for dyadic data analysis, the basis for this technique is regression (Chung et al., 2009; Kenny et al., 2006). With a sample size of 35 dyads, assuming an alpha level of .05, the power of the regression Ftest to detect a significant prediction model for dyadic coping is approximately $0.80 \mathrm{in}$ the presence of a medium effect size ${ }^{33}$. Similarly, with a sample size of 51 dyads,

\footnotetext{
${ }^{33}$ Before conducting the power analysis to support the decision to run two separate analysis by age group, the effect size of the individual dyadic coping style was calculated from the squared multiple correlation of each partner score using the software $G *$ Power 3.1.9.2 Results indicate an average effect size of 0.164 for the younger group and 0.118 for the older group.
} 
assuming an alpha level of .05 , the power of the regression F-test to detect a significant prediction model for dyadic coping is approximately .82 with an effect size of .118 . By adding another variable to the equation, power would have been significantly compromised. Power estimates were obtained using G*Power 3.1.9.2.

As the total dyadic coping score is obtained from the sum of the items included in all the other subscales and does not have a positive or negative implication in terms of individual's and relational adjustment to cancer, it is not presented in this chapter. It was however included in all the analyses. In the overall sample, mean differences by role were identified, with patients reporting significantly higher total dyadic coping scores than caregivers. An average actor effect was also found, indicating that higher mutuality contributes to higher total dyadic coping scores in the study participants. When analyzed in the two groups, mean differences by role and an average actor effect existed for younger dyads, while an overall actor effect identified in the older sample. Hence, among younger dyads it was predicted that patients report higher scores for total dyadic coping than caregivers, and that the individual's score on mutuality predicted patients and caregivers' own total dyadic coping score. Among older couples, only an average actor effect emerged from the analysis, which indicates that the mutuality score reported by the patient and the caregiver was predictive of her/his own total score. 


\section{Stress Communication}

AStress Communication $_{i j}=\left(\mathrm{b}_{0}+\mathrm{d}_{\mathrm{j}}\right)+\mathrm{b}_{1}$ ARole $+\mathrm{b}_{2}$ AMutuality_ $\mathrm{C}_{\mathrm{ij}}+\mathrm{b}_{3}$ PMutuality_ $\mathrm{C}_{\mathrm{ij}}+$ $\mathrm{b}_{4}\left(\right.$ ARole*AMutuality_ $\left.\mathrm{C}_{\mathrm{ij}}\right)+\mathrm{b}_{5}\left(\right.$ ARole*PMutuality_ $\left.\mathrm{C}_{\mathrm{ij}}\right)+e_{i j}$

Multi-level modeling was used to examine whether a person's self-reported level of mutuality and his or her partner's mutuality predicts the individual's engagement in stress communication. Prior to analyzing the data, the actor and partner scores were grand-mean centered and the variable role was coded as 1 for patients, and -1 for caregivers. An Intraclass Correlation Coefficient was calculated on the actor and partner stress communication variables to address their non-independence. Results indicate that the variables are negatively correlated $(\mathrm{ICC}=-.241, p<.05)$ and confirm their interdependence.

It was then tested whether role in the dyad should be treated as a distinguishable factor in the analyses. This distinguishability test resulted in a significant chi-square test, $\left(\chi^{2}(4)=43.72, p<.001\right)$ showing that constraints required for an indistinguishable model significantly worsened the model fit. Thus, dyads were treated as distinguishable, and the chosen residuals structure was heterogeneous compound symmetry ${ }^{34}$.

The results of the APIM analysis are presented in table 27. There is evidence of only an average actor effect of mutuality on stress communication, indicating no significant differences between patients' and caregivers' actor effects. Both patients and caregivers reporting high levels of mutuality were more likely to engage in stress communication behaviors. Specifically, a one unit increase in the mean score of

\footnotetext{
${ }^{34}$ Heterogeneous Compound Symmetry is characterized by non-constant variance and constant correlation. Among the covariance structure possible in dyadic data analysis is the one most often used for distinguishable dyads.
} 
mutuality is associated to a 0.37 unit increase in the person's stress communication mean score. There wasn't evidence that having a partner who reports higher mutuality increases the person's use of stress communication strategies. Furthermore, these results indicate that there was a significant mean level difference between stress communication scores reported by patients and caregivers, with patients reporting higher use of this coping strategy. Finally, the approximate proportion of variance in stress communication explained by actor and partner mutuality was estimated. For patients pseudo $\mathrm{R}^{2}$ was 0.17 , indicating that approximately $17 \%$ of the variance in stress communication was accounted for mutuality and for partners was 0.057 , indicating that approximately $6 \%$ of the variance in stress communication of caregivers was accounted by mutuality.

Table 27. Multilevel regression coefficients estimating actor and partner effects of Mutuality on Stress Communication ${ }^{35}$

\begin{tabular}{lccc}
\hline & $b$ & $\beta$ & $t(d f)$ \\
\hline Intercept & 3.82 & & \\
Role & $\mathbf{0 . 3 9}$ & $\mathbf{0 . 4 7}$ & $\mathbf{7 . 2 0 * * * ( 8 3 )}$ \\
Actor Mutuality & $\mathbf{0 . 3 7}$ & $\mathbf{0 . 3 0}$ & $\mathbf{4 . 3 3 * * * ( 1 5 0 )}$ \\
Partner Mutuality & -0.07 & -0.06 & $-0.82(149)$ \\
Role*Actor Mutuality & 0.09 & 0.07 & $1.13(153)$ \\
Role*Partner Mutuality & 0.05 & 0.04 & $0.64(152)$ \\
\hline
\end{tabular}

Note. Participant role was coded patient $=1$, caregiver $=-1$.

$* p<.05, * * p<.01, * * * p<.001$

${ }^{35}$ All the calculations for the APIM model are available for review in Appendix B. 
Figure 6. The actor and partner effects of Mutuality as predictors of Stress Communication. APIM with distinguishable dyads regression model.

$* p<.05, * * p<.01, * * * p<.001$

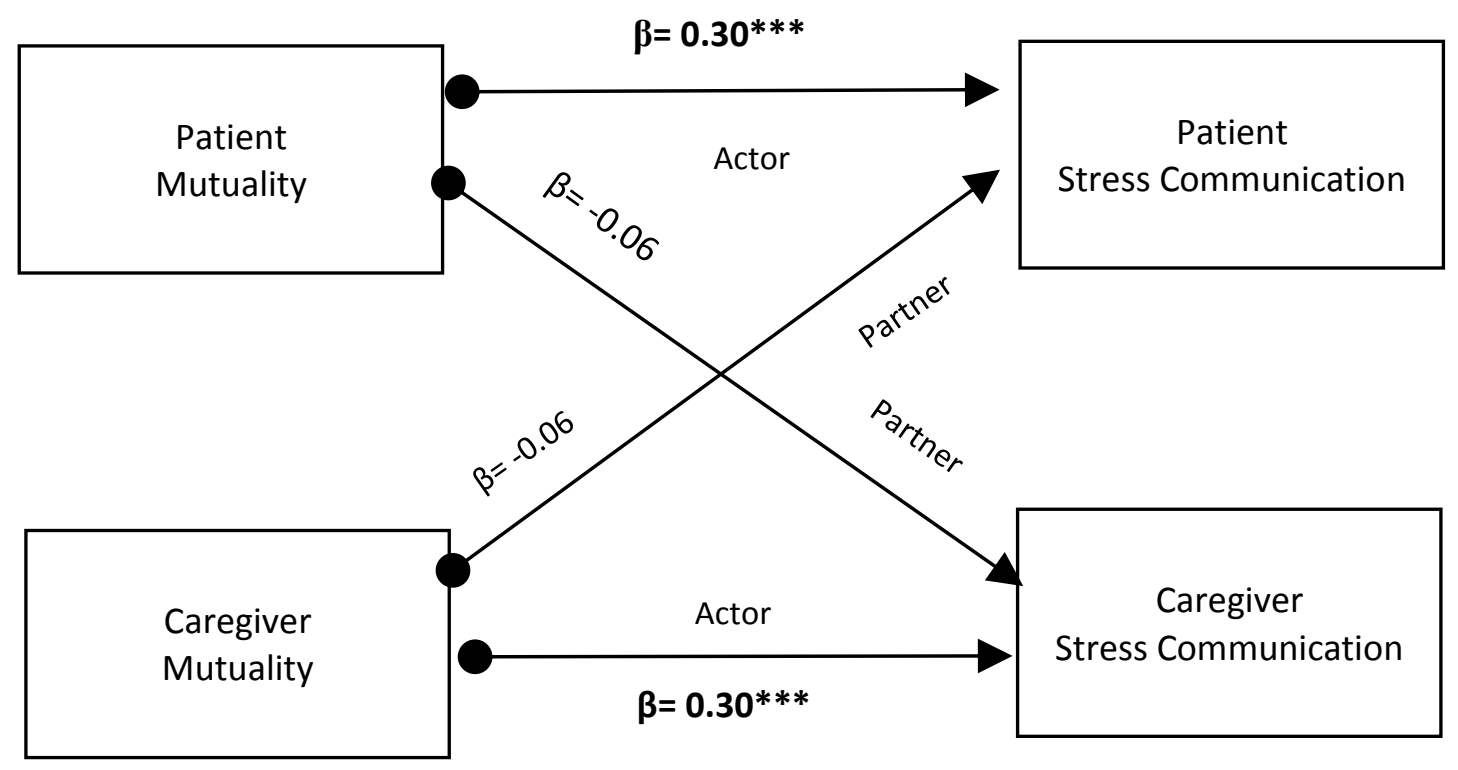

\section{Common Dyadic Coping}

ACommon Dyadic Coping $_{i j}=\left(\mathrm{b}_{0}+\mathrm{d}_{\mathrm{j}}\right)+\mathrm{b}_{1}$ ARole $+\mathrm{b}_{2}$ AMutuality_C $_{\mathrm{ij}}+\mathrm{b}_{3}$

PMutuality_C $\mathrm{Cij}_{\mathrm{ij}}+\mathrm{b}_{4}($ ARole*AMutuality_C $\mathrm{ij})+\mathrm{b}_{5}\left(\right.$ ARole*PMutuality_C $\left.\mathrm{C}_{\mathrm{ij}}\right)+e_{i j}$

This APIM model examined how patients and caregivers' mutuality scores predict the individual's ability to participate in the coping process in a coordinated and symmetrical manner. While the Intraclass Correlation Coefficient was significant $(\mathrm{ICC}=.477, p<.001)$, the Test of Distinguishability wasn't significant $\left(\chi_{(4)}^{2}=7.186\right.$, $p>.05)$, showing that constraints required for an indistinguishable model wouldn't significantly worsen the model fit. However, despite this result, dyads were treated as distinguishable because of the extensive body of literature that identifies differences in the experience of cancer patients and their partners. 
Both an average actor and partner effects of mutuality on common dyadic coping were identified (Table 28). Individuals reporting high levels of mutuality are more engaged in common dyadic coping behaviors as well as individuals whose partners report higher levels of mutuality. Specifically, a one unit increase in the mean score on mutuality is associated with a 0.65 unit increase in common dyadic coping score. Similarly, one unit increase in the mean score on mutuality of the partner contributes to 0.16 unit increase in the individual's score on coping. As a last step in the analysis the approximate proportion of variance in common dyadic coping explained by actor and partner mutuality for patients and caregivers were estimated. Pseudo $\mathrm{R}^{2}$ was 0.55 for patients and 0.39 for caregivers, indicating that mutuality contributes to a large proportion of variance in common dyadic coping.

Table 28. Multilevel regression coefficients estimating actor and partner effects of Mutuality on Common Dyadic Coping

\begin{tabular}{llcc}
\hline & $b$ & $\beta$ & $t(d f)$ \\
\hline Intercept & 3.59 & & \\
Role & 0.01 & 0.01 & $0.34(83)$ \\
Actor Mutuality & $\mathbf{0 . 6 5}$ & $\mathbf{0 . 6 3}$ & $\mathbf{1 1 . 3 1} * *(\mathbf{1 6 5})$ \\
Partner Mutuality & $\mathbf{0 . 1 6}$ & $\mathbf{0 . 1 5}$ & $\mathbf{2 . 8 0} * *(\mathbf{1 6 5})$ \\
Role*Actor Mutuality & 0.10 & 0.10 & $1.65(124)$ \\
Role*Partner Mutuality & 0.04 & 0.04 & $0.75(124)$ \\
\hline
\end{tabular}

Note. Participant role was coded patient $=1$, caregiver $=-1$.

$* p<.05, * * p<.01, * * * p<.001$ 
Figure 7. The actor and partner effects of Mutuality as predictors of Common Dyadic Coping. APIM with distinguishable dyads regression model.

$* p<.05, * * p<.01, * * * p<.001$

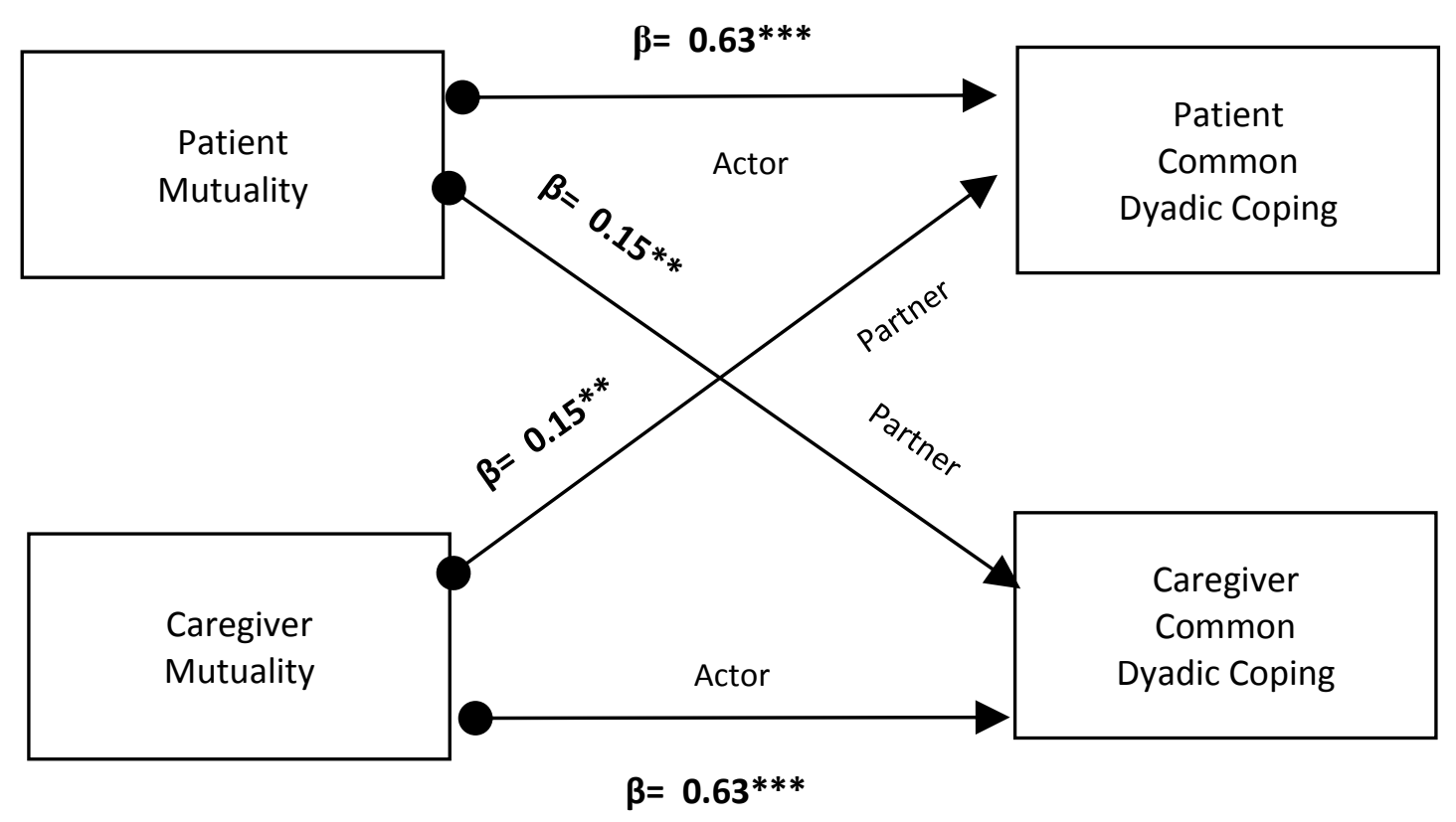

\section{Positive Dyadic Coping}

APositive Dyadic Coping $i j=\left(\mathrm{b}_{0}+\mathrm{d}_{\mathrm{j}}\right)+\mathrm{b}_{1}$ ARole $+\mathrm{b}_{2}$ AMutuality_ $\mathrm{C}_{\mathrm{ij}}+\mathrm{b}_{3}$

PMutuality_C $\mathrm{Cij}_{\mathrm{ij}}+\mathrm{b}_{4}\left(\right.$ ARole*AMutuality_C $\left.\mathrm{C}_{\mathrm{ij}}\right)+\mathrm{b}_{5}\left(\right.$ ARole*PMutuality_C $\left.\mathrm{C}_{\mathrm{ij}}\right)+\mathrm{e}_{\mathrm{ij}}$

APositive Dyadic Coping $i j=\mathrm{b}_{1}$ Patient $+\mathrm{b}_{2}$ Caregiver $+\mathrm{b}_{3}$ Patient*AMutuality_ $\mathrm{C}_{\mathrm{ij}}+$

$\mathrm{b}_{4}$ Caregiver*AMutuality_ $\mathrm{C}_{\mathrm{ij}}+\mathrm{b}_{5}$ Patient*PMutuality_C $\mathrm{C}_{\mathrm{ij}}+\mathrm{b}_{6}$ Caregiver*PMutuality_C + $\mathrm{e}_{1 \mathrm{j}}+\mathrm{e}_{2 \mathrm{j}}$

In this analysis it was examined if the self-reported mutuality score of breast cancer patients and caregivers predicts the engagement in positive dyadic coping. Results of the Intraclass Correlation Coefficient confirmed that the two variables were positively correlated $(\mathrm{ICC}=.39, p<.001)$ and nonindependent, while the Test of Distinguishability confirmed that dyads should be treated as distinguishable $\left(\chi^{2}(4)=14.08, p<.01\right)$. 
As summarized in Table 29, an average actor and partner effects of mutuality on positive dyadic coping were found. Individuals reporting high levels of mutuality are more likely to engage in positive dyadic coping behaviors. Similarly, patients and caregivers whose partner reports higher levels of mutuality are predicted to engage in this coping style. Specifically, a one unit increase in the mean score of mutuality is associated to a 0.76 unit increase in the person's mean of positive dyadic coping, and a one unit increase in mutuality of the partner contributes to 0.13 unit increase in the outcome measure. In the present analysis, mean-level differences of positive dyadic coping of patients and caregivers approached significance $(\mathrm{p}=.053)$.

In addition to these effects, role was a significant moderator of the actor affect, while the interaction between role and the partner effect failed to reach significance. To examine the actor by role interaction, the simple slopes for the actor effect of mutuality were calculated for patients and caregivers by using a two-intercept model approach. Both patients and caregivers' actor effects were statistically significant. However, the effect of mutuality on positive dyadic coping was higher on patients (Table 30).

Finally, the approximate proportion of variance was estimated. For patients pseudo $\mathrm{R}^{2}=.64$ and for caregivers the pseudo $\mathrm{R}^{2}=.40$, indicating that a very large proportion of variance in positive dyadic coping is explained by patients and caregivers' mutuality.

In conclusion, results indicate that an individual's positive dyadic coping is predicted by both the individual and his/her partner's mutuality. Furthermore, role moderated the actor effect of mutuality and results suggest that although both patients and caregivers' own mutuality predicts positive dyadic coping while controlling for their 
partner's mutuality, the patients' actor effect was significantly stronger than the caregivers' actor effect.

Table 29. Multilevel regression coefficients estimating actor and partner effects of Mutuality on Positive Dyadic Coping

\begin{tabular}{lccc}
\hline & $b$ & $\beta$ & $t(d f)$ \\
\hline Intercept & 4.05 & & \\
Role & 0.06 & 0.09 & $1.96(83)$ \\
Actor Mutuality & $\mathbf{0 . 7 6}$ & $\mathbf{0 . 6 8}$ & $\mathbf{1 2 . 9 8 * * * ( 1 6 4 )}$ \\
Partner Mutuality & $\mathbf{0 . 1 3}$ & $\mathbf{0 . 1 1}$ & $\mathbf{2 . 1 3} *(\mathbf{1 6 3})$ \\
Role*Actor Mutuality & $\mathbf{0 . 1 7}$ & $\mathbf{0 . 1 5}$ & $\mathbf{2 . 6 6 * * ( 1 3 2 )}$ \\
Role*Partner Mutuality & 0.01 & 0.01 & $0.10(130)$ \\
\hline
\end{tabular}

Note. Participant role was coded patient $=1$, caregiver $=-1$.

$* p<.05, * * \mathrm{p}<.01, * * * \mathrm{p}<.001$

Table 30. Simple slope regression coefficients estimating separate Mutuality actor and partner effects for patients and caregivers on Positive Dyadic Coping

\begin{tabular}{lccc}
\hline & $b$ & $\beta$ & $t(d f)$ \\
\hline Intercepts & & & \\
Patients & 4.12 & & \\
Caregivers & 3.98 & & \\
Actor's Mutuality & & & $\mathbf{1 1 . 1 8 * * * ( 8 3 )}$ \\
Patients & $\mathbf{0 . 9 3}$ & $\mathbf{0 . 8 3}$ & $\mathbf{6 . 6 6}^{* * *(83)}$ \\
Caregivers & $\mathbf{0 . 5 9}$ & $\mathbf{0 . 5 3}$ & $1.61(83)$ \\
Partner's Mutuality & & & $1.31(83)$ \\
Patients & 0.13 & 0.12 & \\
Caregivers & 0.12 & 0.11 &
\end{tabular}

Note. ${ }^{*} p<.05, * * \mathrm{p}<.01, * * * \mathrm{p}<.001$ 
Figure 8. The actor and partner effects of Mutuality as predictors of Positive Dyadic Coping. APIM with distinguishable dyads regression model.

$* p<.05, * * p<.01, * * * p<.001$

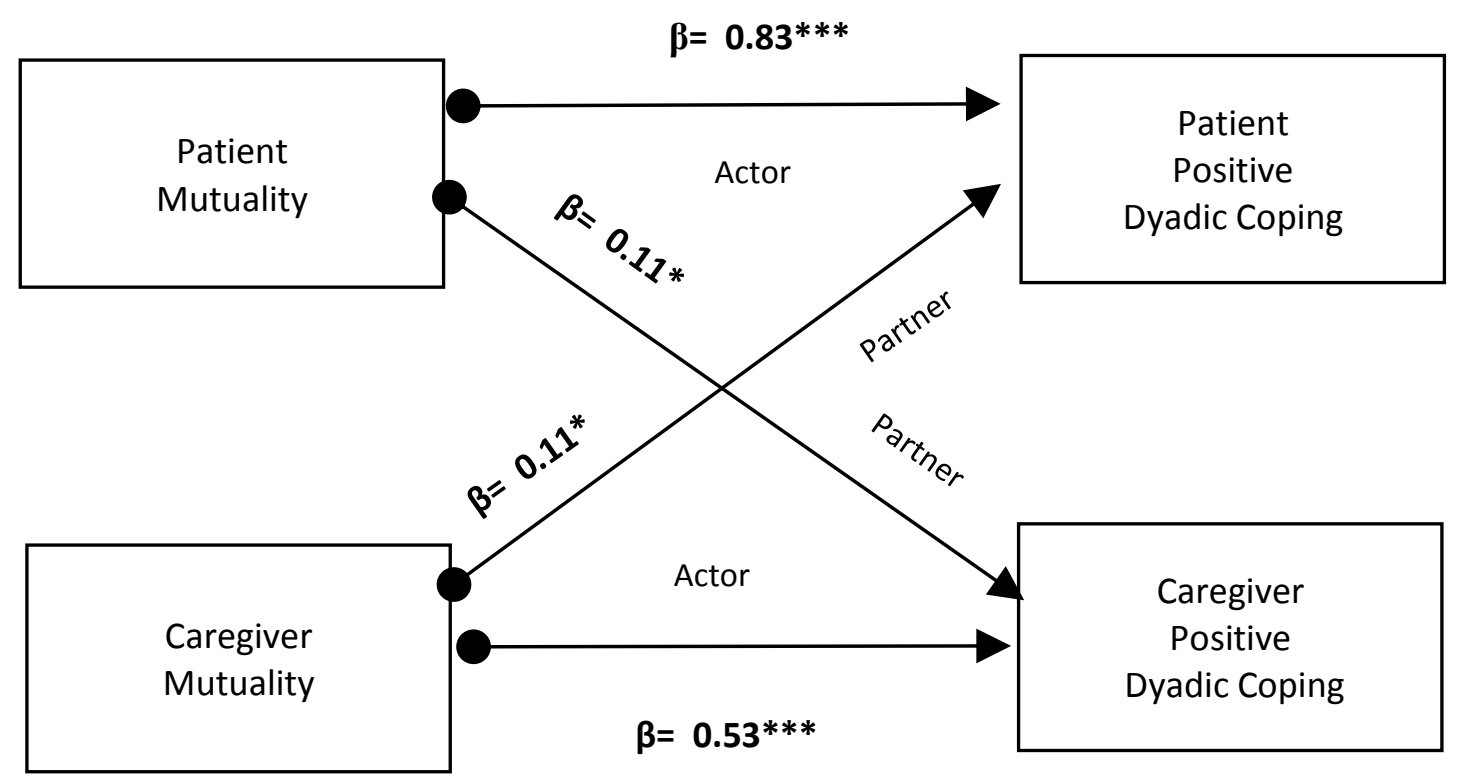

\section{Hostile Dyadic Coping}

AHostile Dyadic Coping $_{i j}=\left(\mathrm{b}_{0}+\mathrm{d}_{\mathrm{j}}\right)+\mathrm{b}_{1}$ ARole $+\mathrm{b}_{2}$ AMutuality_C $\mathrm{C}_{\mathrm{ij}}+\mathrm{b}_{3}$ PMutuality_C $\mathrm{C}_{\mathrm{ij}}+$

$$
\text { b4 (ARole*AMutuality_C } \left.\mathrm{C}_{\mathrm{ij}}\right)+\mathrm{b}_{5}\left(\text { ARole*PMutuality_C } \mathrm{C}_{\mathrm{ij}}\right)+\mathrm{e}_{\mathrm{ij}}
$$

AHostile Dyadic Coping $i j=\mathrm{b}_{1}$ Patient $+\mathrm{b}_{2}$ Caregiver $+\mathrm{b}_{3}$ Patient*AMutuality_ $\mathrm{C}_{\mathrm{ij}}+$ $\mathrm{b}_{4}$ Caregiver*AMutuality_ $\mathrm{C}_{\mathrm{ij}}+\mathrm{b}_{5}$ Patient*PMutuality_ $\mathrm{C}_{\mathrm{ij}}+\mathrm{b}_{6}$ Caregiver*PMutuality_C +

$$
e_{1 j}+e_{2 j}
$$

The Actor-Partner Interdependence Model was used to examine whether a person's self-reported level of mutuality and his or her partner's mutuality predict the individual's engagement in hostile dyadic coping behaviors. The Intraclass Correlation Coefficient was not significant (ICC $=.150, \mathrm{p}=.16$ ), suggesting a non-significant level of association for actor and partner variables. The Distinguishability Test resulted in a 
significant chi-square test $\left(\chi^{2}(4)=16.94, p<.01\right)$ and dyads were then treated as distinguishable.

Results indicate the presence of an average actor effect of mutuality on hostile dyadic coping (Table 31). Both patients and caregivers reporting high levels of mutuality are less likely to engage in hostile dyadic coping behaviors, but this is not replicated when considering their partners' mutuality score. Specifically, a one unit increase in mutuality is associated to a 0.44 unit decrease in the person's hostile dyadic coping mean score. In addition to this effect, role was a significant moderator of the actor and partner effects of mutuality. To examine these interactions, the simple slope regression coefficients for patients and caregivers were calculated. As shown in Table 32, both patients and caregivers' actor effects were statistically significant, but the effect of mutuality on hostile dyadic coping was higher on patients. Among the partner effects, only the one for caregivers was significant.

Finally, the approximate proportion of variance in hostile dyadic coping explained by actor and partner effect of mutuality for patients and caregivers was estimated. For patients pseudo $\mathrm{R}^{2}=.33$ and for caregivers the pseudo $\mathrm{R}^{2}=.28$, indicating that actor and partner effects for mutuality accounted for a large proportion of variance in hostile dyadic coping.

In conclusion, results indicate that hostile dyadic coping is predicted by the individual's mutuality score of both patients and caregivers. Furthermore, role moderated the actor and partner effects of mutuality and results suggest that although both patients and caregivers' own mutuality predicts reduced hostile dyadic coping, the patient's actor effect was significantly stronger than the caregiver's. The partner effect of mutuality was 
significant only for caregivers, indicating that increases in the mutuality scores of patients were associated to lower levels of hostile dyadic coping in the caregivers. More simply, the patients' perceived mutuality in the relationship is predicted to reduce their own and their partners' hostile dyadic coping score. But the caregiver's perceived mutuality only affected his own score on this coping style.

Table 31. Multilevel regression coefficients estimating actor and partner effects of Mutuality on Hostile Dyadic Coping

\begin{tabular}{lccc}
\hline & $b$ & $\beta$ & $t(d f)$ \\
\hline Intercept & 1.95 & & \\
Role & 0.01 & 0.02 & $.41(83)$ \\
Actor Mutuality & $\mathbf{- 0 . 4 4}$ & $\mathbf{- 0 . 5 0}$ & $\mathbf{- 7 . 4 4 * * * ( 1 3 9 )}$ \\
Partner Mutuality & -0.06 & -0.07 & $-1.09(140)$ \\
Role*Actor Mutuality & $\mathbf{- 0 . 1 6}$ & $\mathbf{- 0 . 1 8}$ & $\mathbf{- 2 . 7 6 * * ( 1 4 5 )}$ \\
Role*Partner Mutuality & $\mathbf{0 . 1 3}$ & $\mathbf{0 . 1 5}$ & $\mathbf{2 . 2 0 * ( 1 4 7 )}$ \\
\hline
\end{tabular}

Note. Participant role was coded patient $=1$, caregiver $=-1$.

$* p<.05, * * \mathrm{p}<.01, * * * \mathrm{p}<.001$

Table 32. Simple slope regression coefficients estimating separate mutuality actor and partner effects for patients and caregivers on Hostile Dyadic Coping

\begin{tabular}{lccc}
\hline & $b$ & $\beta$ & $t(d f)$ \\
\hline Intercepts & & & \\
Patients & 1.97 & & \\
Caregivers & 1.94 & & \\
Actor's Mutuality & & & \\
Patients & $\mathbf{0 . 6 1}$ & $\mathbf{- 0 . 6 9}$ & $\mathbf{- 6 . 3 6 * * * ( 8 3 )}$ \\
Caregivers & $\mathbf{- 0 . 2 8}$ & $\mathbf{- 0 . 3 2}$ & $\mathbf{- 4 . 0 0 * * * ( 8 3 )}$ \\
Partner's Mutuality & & & $\mathbf{0 . 6 8}(\mathbf{8 3})$ \\
Patients & $\mathbf{0 . 0 6}$ & $\mathbf{0 . 0 7}$ & $\mathbf{- 2 . 7 0 * * ( 8 3 )}$ \\
Caregivers & $\mathbf{- 0 . 1 9}$ & $\mathbf{- 0 . 2 2}$ & \\
\hline
\end{tabular}

Note. ${ }^{*} p<.05$ 
Figure 9. The actor and partner effects of Mutuality as predictors of Hostile Dyadic Coping. APIM with distinguishable dyads regression model.

$* p<.05, * * p<.01, * * * p<.001$.

$$
\beta=-0.69 * * *
$$

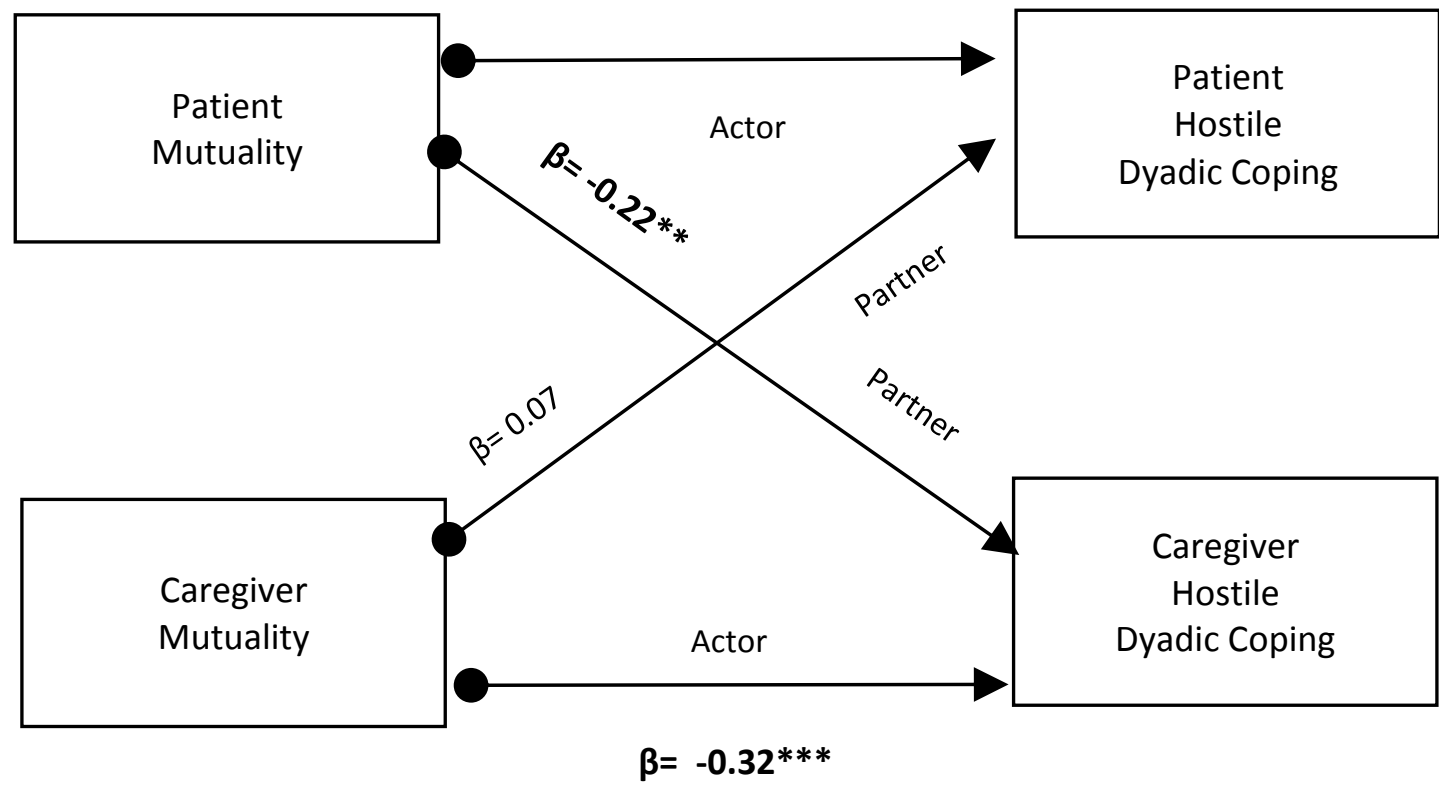

\section{Avoidance of Dyadic Coping}

AAvoidance of Dyadic Coping $i j=\left(\mathrm{b}_{0}+\mathrm{d}_{\mathrm{j}}\right)+\mathrm{b}_{1}$ ARole $+\mathrm{b}_{2}$ AMutuality_ $\mathrm{C}_{\mathrm{ij}}+\mathrm{b}_{3}$ PMutuality_C $\mathrm{Cij}_{\mathrm{ij}}+\mathrm{b}_{4}\left(\right.$ ARole*AMutuality_C $\left.\mathrm{ij}_{\mathrm{ij}}\right)+\mathrm{b}_{5}\left(\right.$ ARole*PMutuality_C $\left.\mathrm{C}_{\mathrm{ij}}\right)+\mathrm{e}_{\mathrm{ij}}$

Avoidance of dyadic coping is observed when the coping responses of one partner are ambivalent and superficial, and therefore authentic engagement is absent. The degree to which a person's self-reported level of mutuality and the partner's mutuality predict the individual's avoidance of dyadic coping was investigated using multilevel modeling. The Intraclass Correlation Coefficient only approached significance $(\mathrm{ICC}=.196, p=.06)$, and a significant chi square analysis $\left(\chi^{2}(4)=10.52, p<.05\right)$ confirmed that dyads could be treated as distinguishable. 
There is evidence of both an actor and partner effect of mutuality on avoidance of dyadic coping (Table 33). Individuals reporting high levels of mutuality are less likely to engage in avoidant dyadic coping behaviors, and the same occurs for patients and caregivers whose partners are higher in mutuality. Specifically, a one unit increase in mutuality is associated to a 0.53 unit decrease in the person's avoidant dyadic coping mean score. Further, every one unit increase in the partner's mean mutuality score is associated with a 0.19 reduction in the avoidance of dyadic coping. Role was not a significant moderator of the actor and partner effects, as the interaction between role and these two effects failed to reach significance.

The approximate proportion of variance in avoidance of dyadic coping explained by actor and partner mutuality for patients and caregivers was estimated. For patients pseudo $R^{2}=0.24$ and for caregivers the pseudo $R^{2}=0.27$. Thus the actor and partner effects for mutuality accounted for a large proportion of the variance in avoidance of dyadic coping.

In conclusion, results indicate that an individual's avoidance of dyadic coping is predicted by the self-reported score on mutuality, as well as by the partner's. Furthermore, role did not moderate the actor and partner effects of mutuality, indicating that no significant differences exist in the actor and partner effects of patients and caregivers. 
Table 33. Multilevel regression coefficients estimating actor and partner effects of Mutuality on Avoidance of Dyadic Coping

\begin{tabular}{lccc}
\hline & $b$ & $\beta$ & $t(d f)$ \\
\hline Intercept & 2.64 & & \\
Role & 0.05 & 0.06 & $1.02(83)$ \\
Actor Mutuality & $\mathbf{- 0 . 5 3}$ & $\mathbf{- 0 . 4 2}$ & $\mathbf{- 6 . 0 3 * * * ( 1 4 2 )}$ \\
Partner Mutuality & $\mathbf{- 0 . 1 9}$ & $\mathbf{- 0 . 1 6}$ & $\mathbf{- 2 . 2 1 * ( 1 4 4 )}$ \\
Role*Actor Mutuality & -0.12 & -0.10 & $-1.42(141)$ \\
Role*Partner Mutuality & 0.09 & 0.07 & $1.02(143)$ \\
\hline
\end{tabular}

Note. Participant role was coded patient $=1$, caregiver $=-1$.

$* p<.05 * * \mathrm{p}<.01, * * * \mathrm{p}<.001$

Figure 10. The actor and partner effects of Mutuality as predictors of Avoidance of Dyadic Coping. APIM with distinguishable dyads regression model.

$* p<.05, * * p<.01, * * * p<.001$

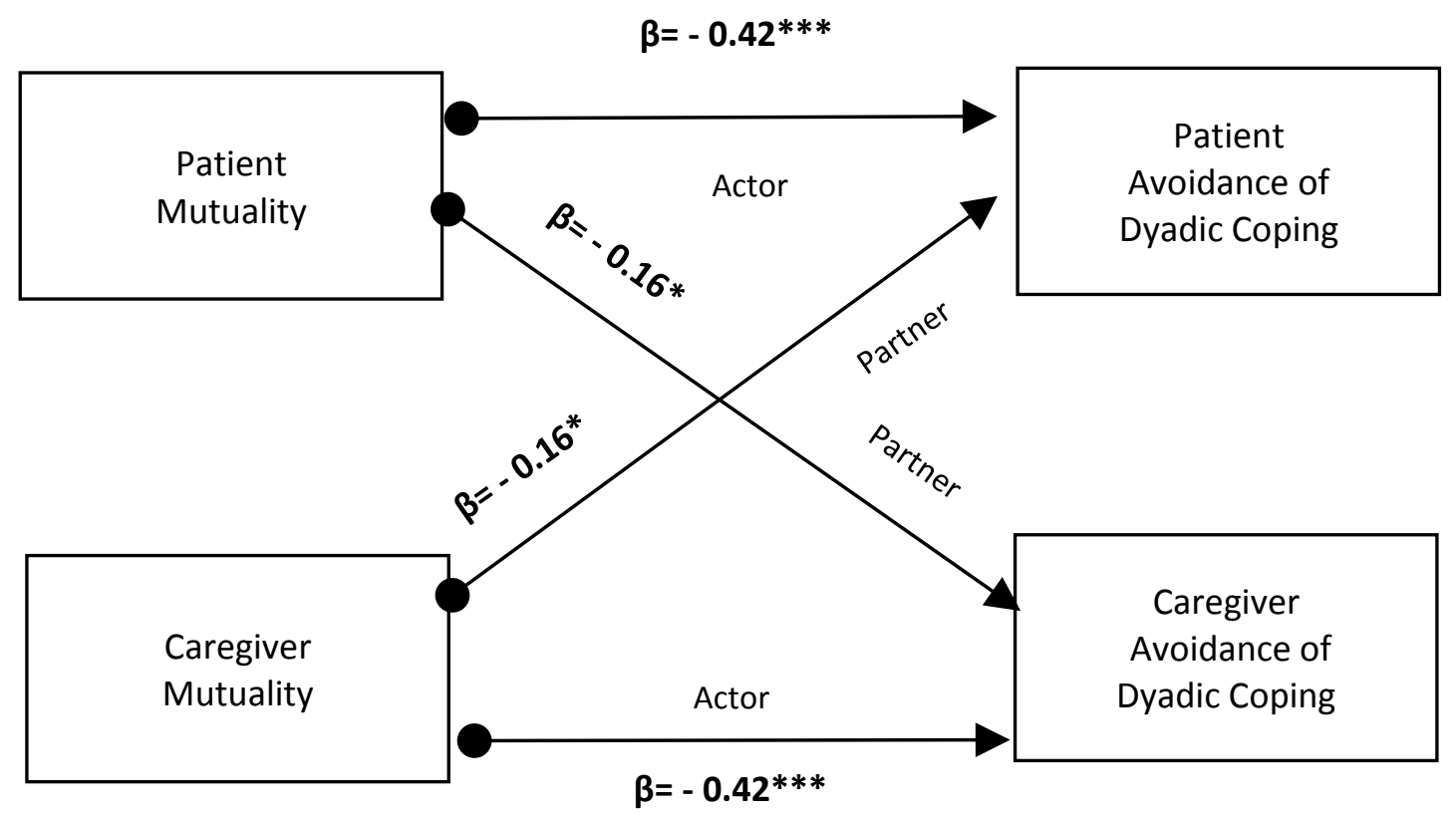




\section{Actor Partner Model by Age Group}

After this initial analysis, separate APIM models were conducted on the younger and older dyads to detect differences in the actor and partner effects. Using the effect coded variable Age Group (-1: Younger Dyads, 1: Older Dyads) the sample was divided in two groups and the same dyadic data analysis was conducted on them.

\section{Stress Communication}

Multi-level modeling was used to examine the degree to which patients and caregivers' mutuality predicts the individual's engagement in stress communication in younger and older dyads. Prior to analyzing the data, an Intraclass Correlation Coefficient and a Test of Distinguishability were conducted. Actor and partner's scores on Stress Communication are negatively correlated (ICC $=-.385, p<.05)$ and nonindependent in the younger sample, while for older couples the ICC was not significant (ICC $=-.145$, $p>.05)$. The distinguishability test resulted in a significant chi-square test for both groups, suggesting that dyads could be treated as distinguishable (Appendix B). The residual structure was, again, heterogeneous compound symmetry.

In both groups there is evidence of only an average actor effect of mutuality on stress communication (see Table 34 and Table 35). Individuals reporting high levels of mutuality were more likely to engage in stress communication behaviors. Specifically, a one unit increase in the mean score of mutuality is associated to a 0.66 unit increase in stress communication for younger participants, while for older patients and caregivers this increase was of 0.25 . There wasn't evidence that having a partner who reported higher mutuality increased the person's use of this coping strategy. Furthermore, mean level differences exist between stress communication by patients and caregivers, with 
younger and older patients reporting higher use of this coping strategy than their respective caregivers.

The approximate proportion of variance in stress communication explained by actor and partner mutuality was estimated. For both younger patients and caregivers pseudo $\mathrm{R}^{2}$ was 0.25 , indicating that a large amount of variance in stress communication was accounted for by mutuality. In the older counterpart, pseudo $\mathrm{R}^{2}$ for patients was 0.108 and the $\mathrm{R}^{2}$ for partners was 0.023 , indicating that approximately $11 \%$ and $2.3 \%$ of the variance in stress communication is accounted for by patients and partners' mutuality respectively.

Table 34. Multilevel regression coefficients estimating actor and partner effects of Mutuality on Stress Communication of Younger Couples

\begin{tabular}{lccc}
\hline & $b$ & $\beta$ & $t(d f)$ \\
\hline Intercept & 3.75 & & \\
Role & $\mathbf{0 . 4 2}$ & $\mathbf{0 . 5 1}$ & $\mathbf{4 . 8 5 * * * ( 3 2 )}$ \\
Actor Mutuality & $\mathbf{0 . 6 6}$ & $\mathbf{0 . 5 4}$ & $\mathbf{4 . 2 9} * * *(\mathbf{5 0})$ \\
Partner Mutuality & -0.22 & -0.18 & $-1.35(45)$ \\
Role*Actor Mutuality & -0.07 & -0.05 & $-0.46(58)$ \\
Role*Partner Mutuality & 0.13 & 0.11 & $0.85(50)$ \\
\hline
\end{tabular}

Note. Participant role was coded patient $=1$, caregiver $=-1$. ${ }^{*} p<.05 * * \mathrm{p}<.01, * * * \mathrm{p}<.001$ 
Figure 11. The actor and partner effects of Mutuality as predictors of Stress Communication in Younger Couples. APIM with distinguishable dyads regression model. * $p<.05, * * p<.01, * * * p<.0001$

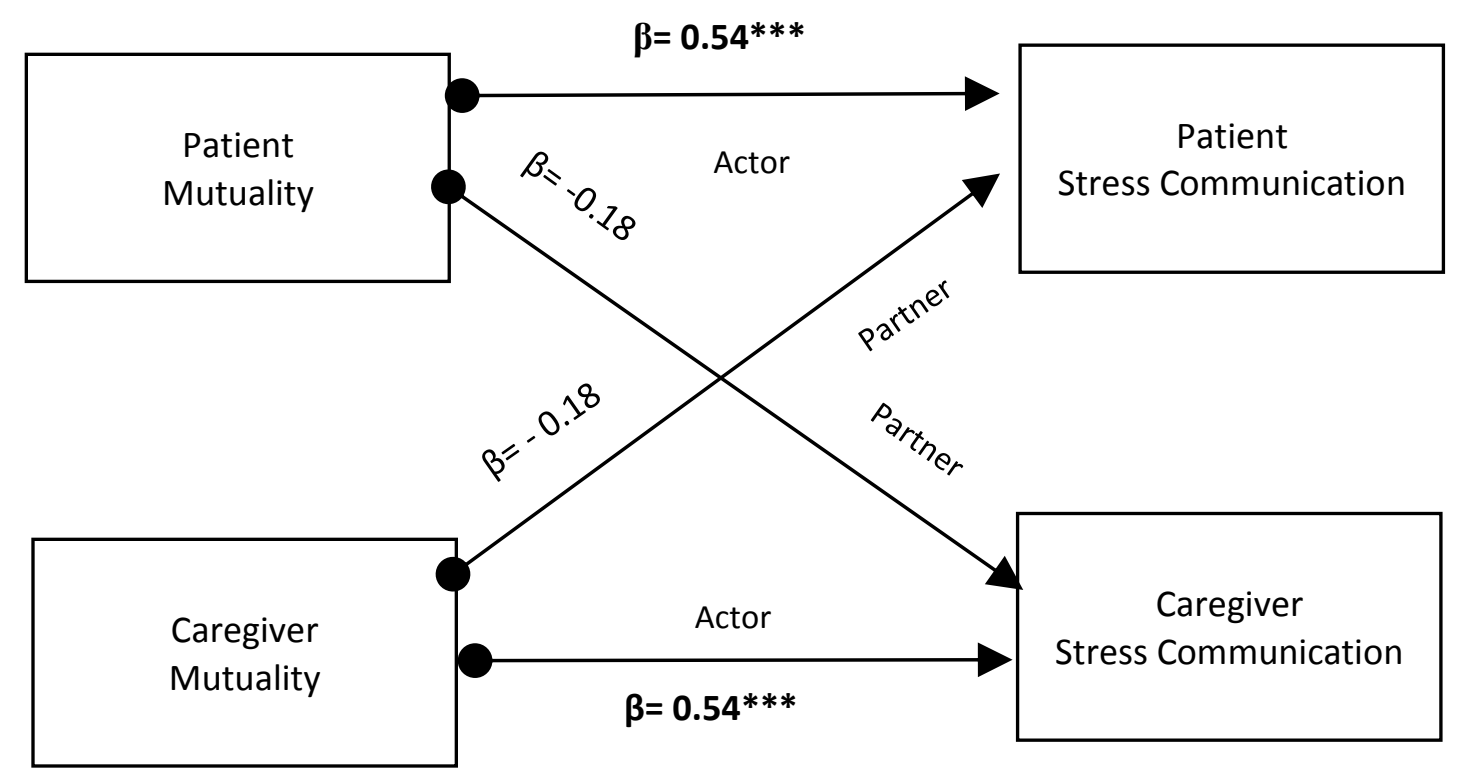


Table 35. Multilevel regression coefficients estimating actor and partner effects of Mutuality on Stress Communication in Older Couples

\begin{tabular}{|c|c|c|c|}
\hline & $b$ & $\beta$ & $t(d f)$ \\
\hline Intercept & 3.86 & & \\
\hline Role & 0.37 & 0.45 & $5.50 * * *(48)$ \\
\hline Actor Mutuality & 0.25 & 0.20 & $2.39 *(91)$ \\
\hline Partner Mutuality & 0.01 & 0.01 & $0.06(82)$ \\
\hline Role*Actor Mutuality & 0.09 & 0.08 & $0.93(85)$ \\
\hline Role*Partner Mutuality & 0.01 & 0.01 & $0.02(77)$ \\
\hline
\end{tabular}

Note. Participant role was coded patient $=1$, caregiver $=-1$

${ }^{*} p<.05 * * \mathrm{p}<.01, * * * \mathrm{p}<.001$

Figure 12. The actor and partner effects of Mutuality as predictors of Stress Communication in Older Couples. APIM with distinguishable dyads regression model. $* \mathrm{p}<.05, * * \mathrm{p}<.01, * * * \mathrm{p}<.0001$

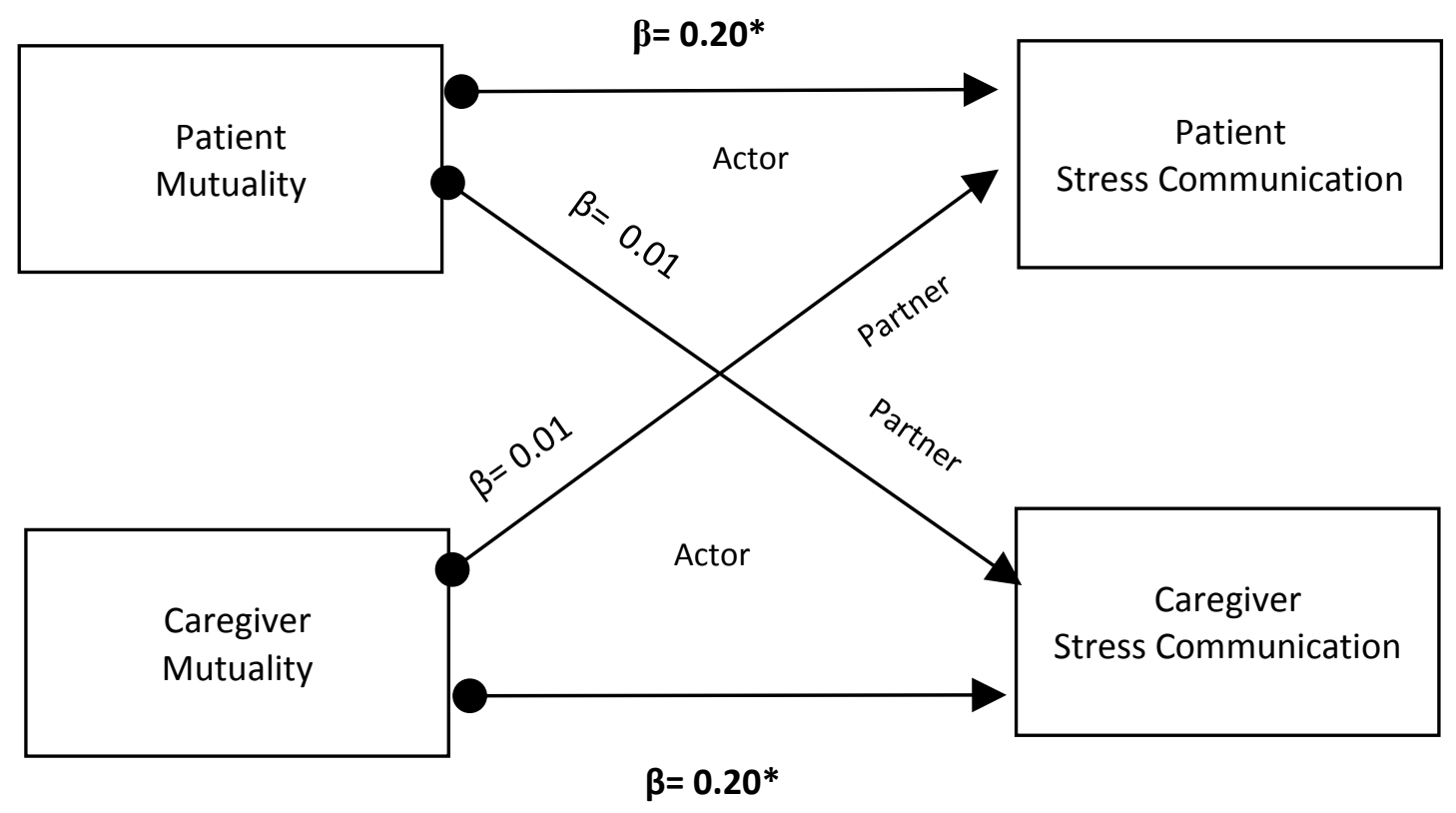




\section{Common Dyadic Coping}

The next analysis examined actor and partner effects of mutuality on common dyadic coping behaviors. The Intraclass Correlation Coefficient indicates that actor and partner's scores on this type of dyadic coping are nonindependent in each group (Younger Dyads: ICC $=.52, p<.01$; Older Dyads: ICC $=.44, p<.01$ ). The distinguishability test resulted in a significant chi-square test for the younger group, while the results of the distinguishability test failed to reach significance for older participants (Appendix B). Despite this, older dyads were treated as distinguishable on the basis of the literature available about couples coping with cancer-related stress. A heterogeneous compound symmetry structure was used to organize residuals.

The results of the APIM analysis for younger and older dyads are presented in Table 36 and 37. Differences exist when comparing the multilevel regression coefficients. Among younger dyads, the analysis revealed the presence of an average actor and partner effect of mutuality on common dyadic coping. Younger patients and caregivers reporting high levels of mutuality were more likely to engage in common dyadic coping behaviors. Specifically, a one unit increase in the mean score of mutuality is associated to a 0.70 unit increase in common dyadic coping. There was also evidence that having a partner who scores high on mutuality increases the person's use of common coping strategies. A one unit increase in the mean score of mutuality reported by the partner is associated to a 0.25 unit increase. On the contrary, among older dyads only an average actor effect was found, indicating that a one unit increase in mutuality of both patients and caregivers was associated to a .65 unit increase in the common dyadic coping score. 
Finally, the approximate proportion of variance in common dyadic coping explained by actor and partner mutuality was estimated. For younger patients and caregivers pseudo $\mathrm{R}^{2}$ were .55 and .48 respectively, indicating that a very large proportion of variance was explained by mutuality. In the older counterpart, pseudo $\mathrm{R}^{2}$ for patients was 0.57 and the $\mathrm{R}^{2}$ for partners was 0.36 , indicating that approximately $57 \%$ and $36 \%$ of the variance in common dyadic coping is accounted for by patients and partners' mutuality.

Table 36. Multilevel regression coefficients estimating Actor and Partner effects of Mutuality on Common Dyadic Coping scores of Younger Couples

\begin{tabular}{lccc}
\hline & $b$ & $\beta$ & $t(d f)$ \\
\hline Intercept & 3.52 & & \\
Role & 0.04 & 0.05 & $1.10(32)$ \\
Actor Mutuality & $\mathbf{0 . 6 9}$ & $\mathbf{0 . 6 6}$ & $\mathbf{7 . 4 5 * * * ( 6 3 )}$ \\
Partner Mutuality & $\mathbf{0 . 2 5}$ & $\mathbf{0 . 2 4}$ & $\mathbf{2 . 3 5 * ( 4 5 )}$ \\
Role*Actor Mutuality & -0.04 & -0.04 & $-0.39(44)$ \\
Role*Partner Mutuality & 0.21 & 0.20 & $1.79(39)$ \\
\hline
\end{tabular}

Note. Participant role was coded patient $=1$, caregiver $=-1$.

$* \mathrm{p}<.05, * * \mathrm{p}<.01, * * * \mathrm{p}<.001$

Figure 13. The actor and partner effects of Mutuality as predictors of Common Dyadic Coping in Younger Couples. APIM with distinguishable dyads regression model. $* p<.05, * * p<.01, * * * p<.001$

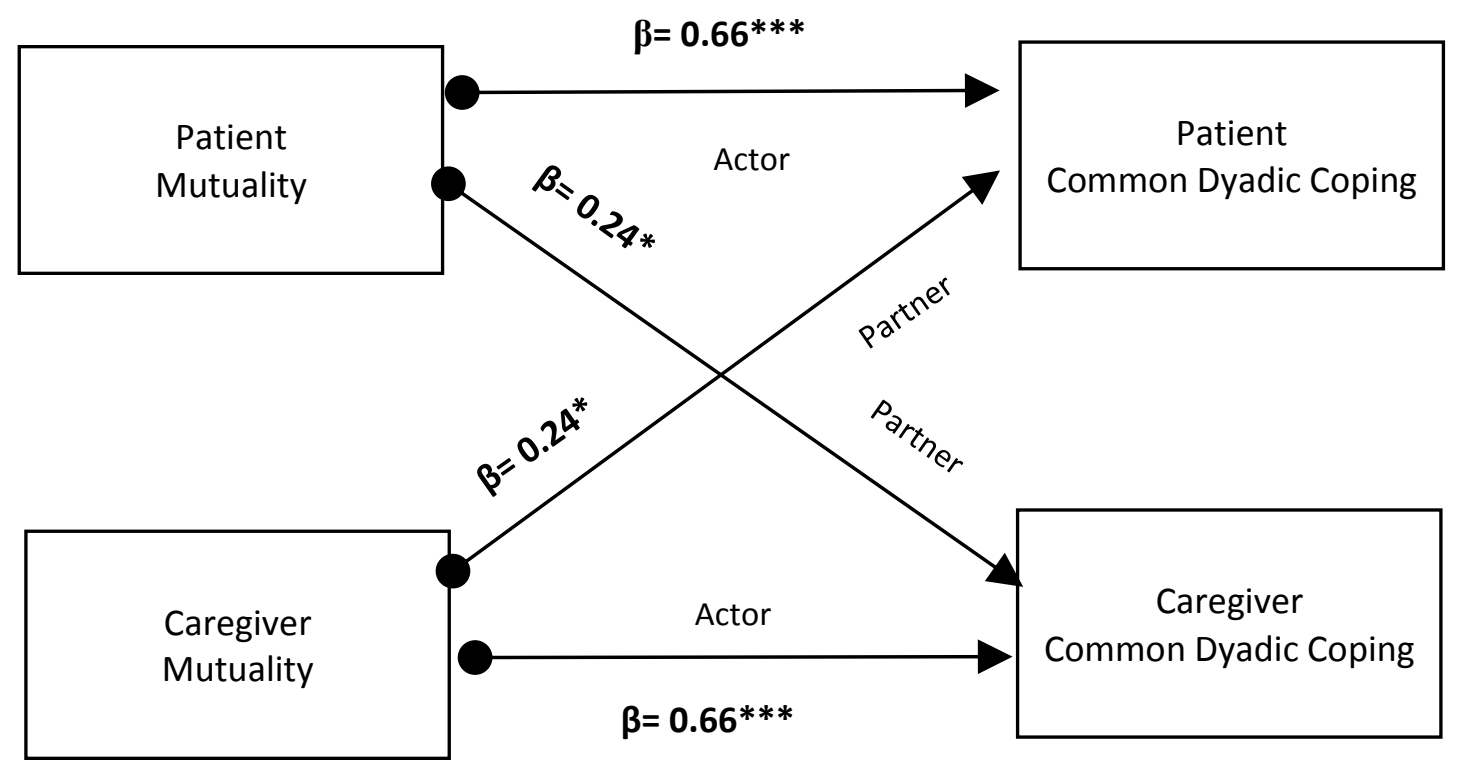


Table 37. Multilevel regression coefficients estimating actor and partner effects of Mutuality on Common Dyadic Coping in Older Couples

\begin{tabular}{lccl}
\hline & $b$ & $\beta$ & $t(d f)$ \\
\hline Intercept & 3.63 & & \\
Role & 0.02 & 0.02 & $0.28(48)$ \\
Actor Mutuality & $\mathbf{0 . 6 5}$ & $\mathbf{0 . 6 3}$ & $\mathbf{8 . 7 1 * * * ( 9 5 )}$ \\
Partner Mutuality & 0.14 & 0.14 & $1.88(86)$ \\
Role*Actor Mutuality & 0.16 & 0.15 & $1.95(74)$ \\
Role*Partner Mutuality & 0.01 & 0.01 & $0.02(70)$ \\
\hline
\end{tabular}

Note. Participant role was coded patient $=1$, caregiver $=-1$.

$* p<.05, * * \mathrm{p}<.01, * * * \mathrm{p}<.001$

Figure 14. The actor and partner effects of Mutuality as predictors of Common Dyadic Coping in Older Couples. APIM with distinguishable dyads regression model.

$* \mathrm{p}<.05, * * \mathrm{p}<.01, * * * \mathrm{p}<.001$

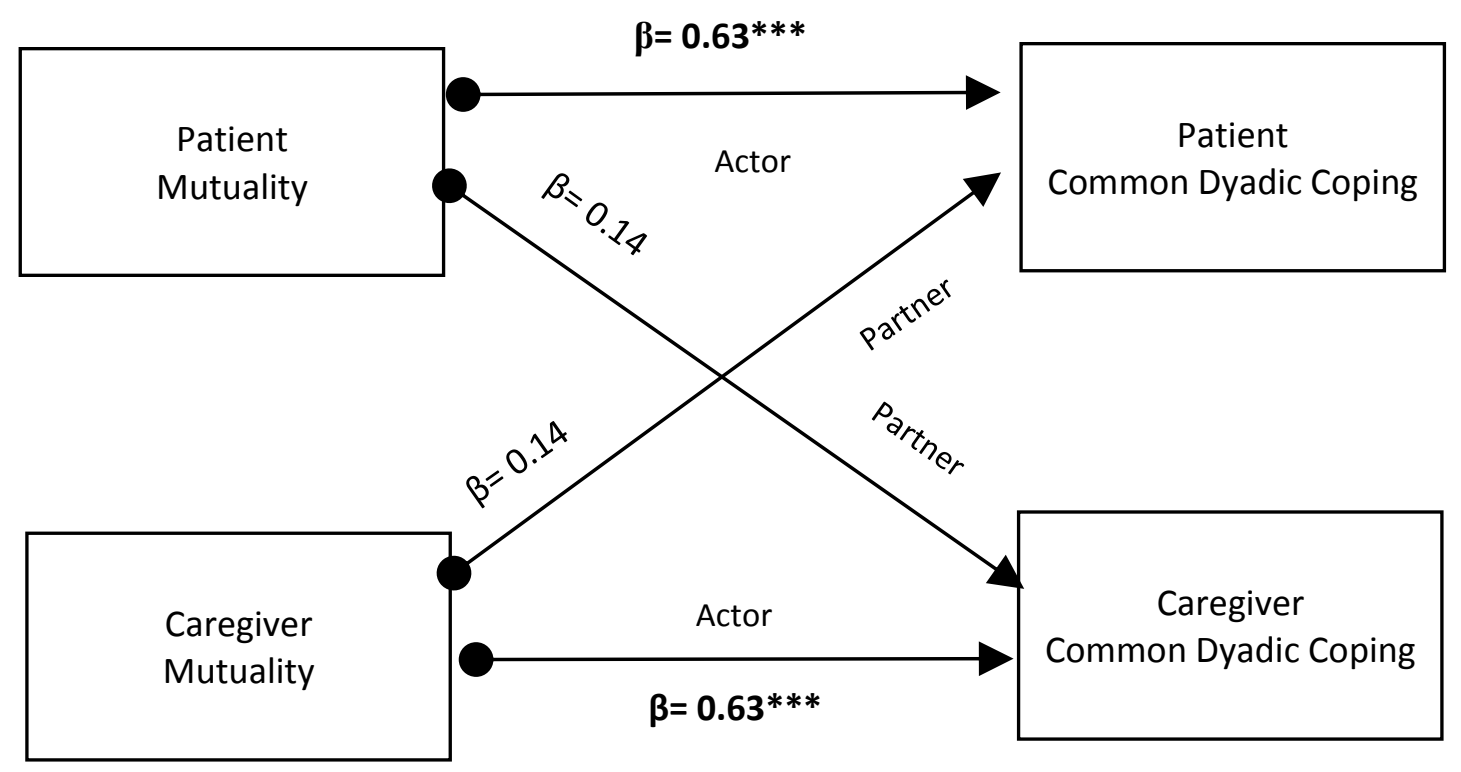

\section{Positive Dyadic Coping}

The next analysis focused on positive dyadic coping. Prior to analyzing the data, an

Intraclass Correlation Coefficient and a Test of Distinguishability were calculated.

Results indicate that actor and partner's scores on this type of dyadic coping are

nonindependent in each group (Younger Dyads: $\mathrm{ICC}=.38, p<.05$; Older Dyads: $\mathrm{ICC}=.38$, $p<.05)$. The distinguishability test was significant only for the younger group $\left(\chi^{2}(4)\right.$ 
$=11.99, p<.05)$. Older dyads were however treated as distinguishable because of the literature that supports differences between patients and partners.

The results of the APIM analysis for younger and older dyads are presented in Tables 38 - 40. Among younger couples, the performed analysis revealed the presence of an average actor effect of mutuality on positive dyadic coping. Younger individuals reporting high levels of mutuality were more likely to engage in positive dyadic coping behaviors. Specifically, a one unit increase in the mean score of mutuality is associated to a 0.89 unit increase in positive dyadic coping. Furthermore, among younger couples significant role differences exist, with patients predicted to report higher scores than their caregivers. In contrast, among older couples there was evidence of an actor effect and of a significance interaction of actor effect by role. Higher self-reported mutuality scores contributed to a 0.73 unit increase in positive dyadic coping. A simple slope model was estimated using a two intercept approach to test whether the actor effect is different by patients and partners (Table 40). Results indicate that among older couples the actor effect was greater for patients.

Finally, the approximate proportion of variance was estimated. For both groups the values were very high, suggesting that mutuality and positive dyadic coping items may be measuring similar constructs. For younger patients and caregivers pseudo $\mathrm{R}^{2}$ were extremely high: .69 and .54 respectively, indicating that a large proportion of variance is explained by mutuality of younger patients and partners. In the older counterpart, pseudo $\mathrm{R}^{2}$ for patients was 0.68 and the $\mathrm{R}^{2}$ for partners was 0.69 , indicating that approximately $68 \%$ and $69 \%$ of the variance in positive dyadic coping is accounted for by patients and 
partners' mutuality. This finding can be explained by the large correlation existing between mutuality and positive dyadic coping.

Table 38. Multilevel regression coefficients estimating actor and partner effects of Mutuality on Positive Dyadic Coping in Younger Couples

\begin{tabular}{lccc}
\hline & $b$ & $\beta$ & $t(d f)$ \\
\hline Intercept & 4.00 & & \\
Role & $\mathbf{0 . 1 3}$ & $\mathbf{0 . 1 8}$ & $\mathbf{3 . 2 9} * *(\mathbf{3 2})$ \\
Actor Mutuality & $\mathbf{0 . 8 9}$ & $\mathbf{0 . 8 0}$ & $\mathbf{9 . 9 7 * * * ( 6 1 )}$ \\
Partner Mutuality & 0.07 & 0.06 & $0.73(49)$ \\
Role*Actor Mutuality & 0.06 & 0.05 & $0.61(44)$ \\
Role*Partner Mutuality & 0.02 & 0.01 & $0.14(41)$ \\
\hline
\end{tabular}

Note. Participant role was coded patient $=1$, caregiver/spouse $=-1$.

$* p<.05 * * \mathrm{p}<.01, * * * \mathrm{p}<.001$

Figure 15. The actor and partner effects of Mutuality as predictors of Positive Dyadic Coping in Younger Couples. APIM with distinguishable dyads regression model. $* p<.05, * * p<.01, * * * p<.001$

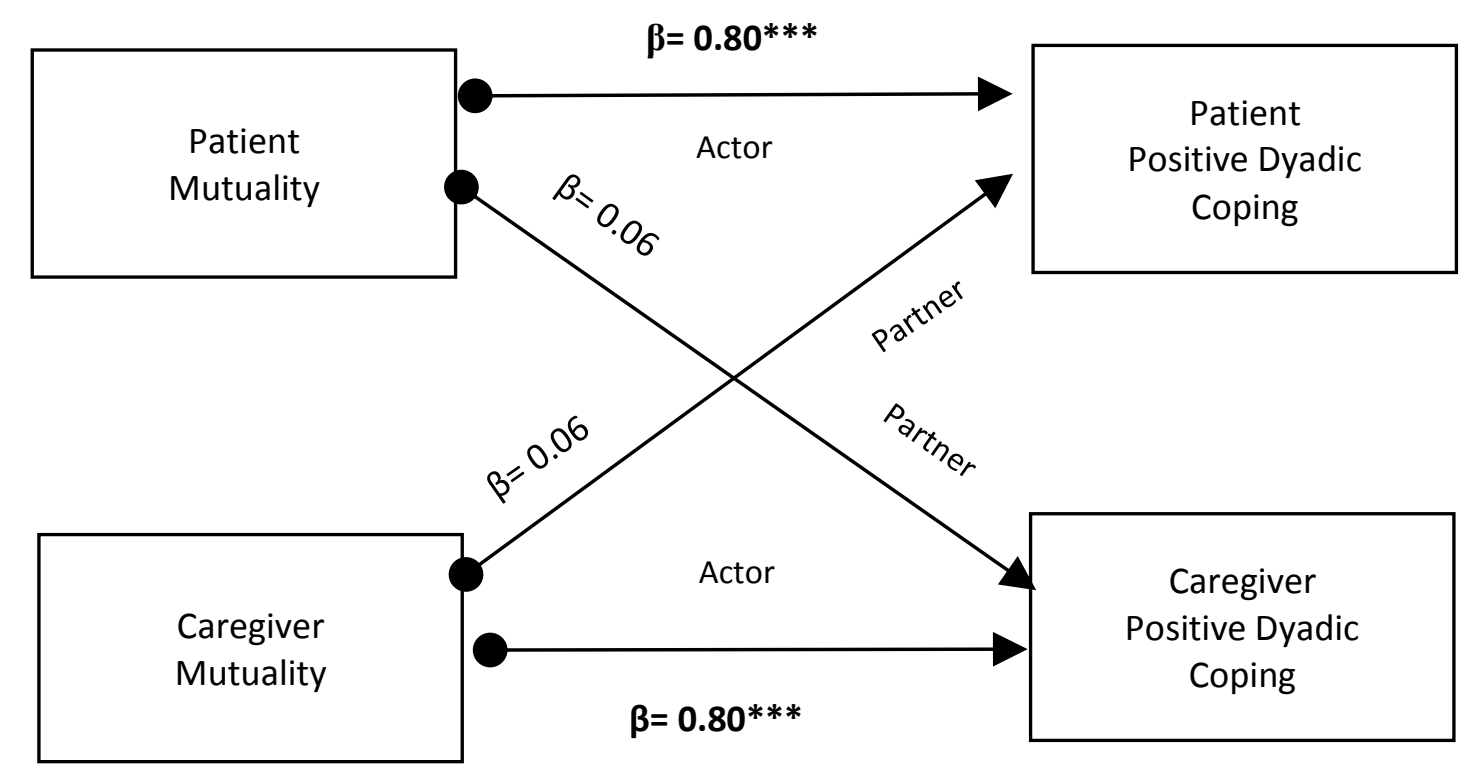


Table 39. Multilevel regression coefficients estimating actor and partner effects of Mutuality on Positive Dyadic Coping in Older Couples

\begin{tabular}{lccl}
\hline & $b$ & $\beta$ & $t(d f)$ \\
\hline Intercept & 4.07 & & \\
Role & 0.02 & 0.03 & $0.42(48)$ \\
Actor Mutuality & $\mathbf{0 . 7 3}$ & $\mathbf{0 . 6 5}$ & $\mathbf{9 . 2 1} * * *(\mathbf{9 5})$ \\
Partner Mutuality & 0.14 & 0.12 & $1.67(84)$ \\
Role*Actor Mutuality & $\mathbf{0 . 1 9}$ & $\mathbf{0 . 1 7}$ & $\mathbf{2 . 2 8 * ( 7 8 )}$ \\
Role*Partner Mutuality & 0.01 & 0.01 & $0.10(73)$ \\
\hline
\end{tabular}

Note. Participant role was coded patient $=1$, caregiver $=-1$

${ }^{*} p<.05^{* *} \mathrm{p}<.01, * * * \mathrm{p}<.001$

Table 40. Simple slope regression coefficients estimating separate mutuality actor and partner effects for patients and caregivers on Positive Dyadic Coping in Older Couples

\begin{tabular}{lccc}
\hline & $b$ & $\beta$ & $t(d f)$ \\
\hline Intercepts & & & \\
Patients & 4.11 & & \\
Caregivers & 4.08 & & \\
Actor's Mutuality & & & $\mathbf{7 . 8 1 * * * ( 4 8 )}$ \\
Patients & $\mathbf{0 . 9 2}$ & $\mathbf{0 . 8 2}$ & $\mathbf{4 . 7 4 * * * ( 4 8 )}$ \\
Caregivers & $\mathbf{0 . 5 4}$ & $\mathbf{0 . 4 8}$ & $1.49(48)$ \\
Partner's Mutuality & & & $0.94(48)$ \\
Patients & 0.14 & 0.13 & \\
Caregivers & 0.13 & 0.12 & \\
\hline No $p<.05 * *<.01, * * *<.001$ & & & \\
\hline
\end{tabular}

Note. ${ }^{*} p<.05^{* *} \mathrm{p}<.01, * * * \mathrm{p}<.001$

Figure 16. The actor and partner effects of Mutuality as predictors of Positive Dyadic Coping in Older Couples. APIM with distinguishable dyads regression model.

$* \mathrm{p}<.05, * * \mathrm{p}<.01, * * * \mathrm{p}<.001$

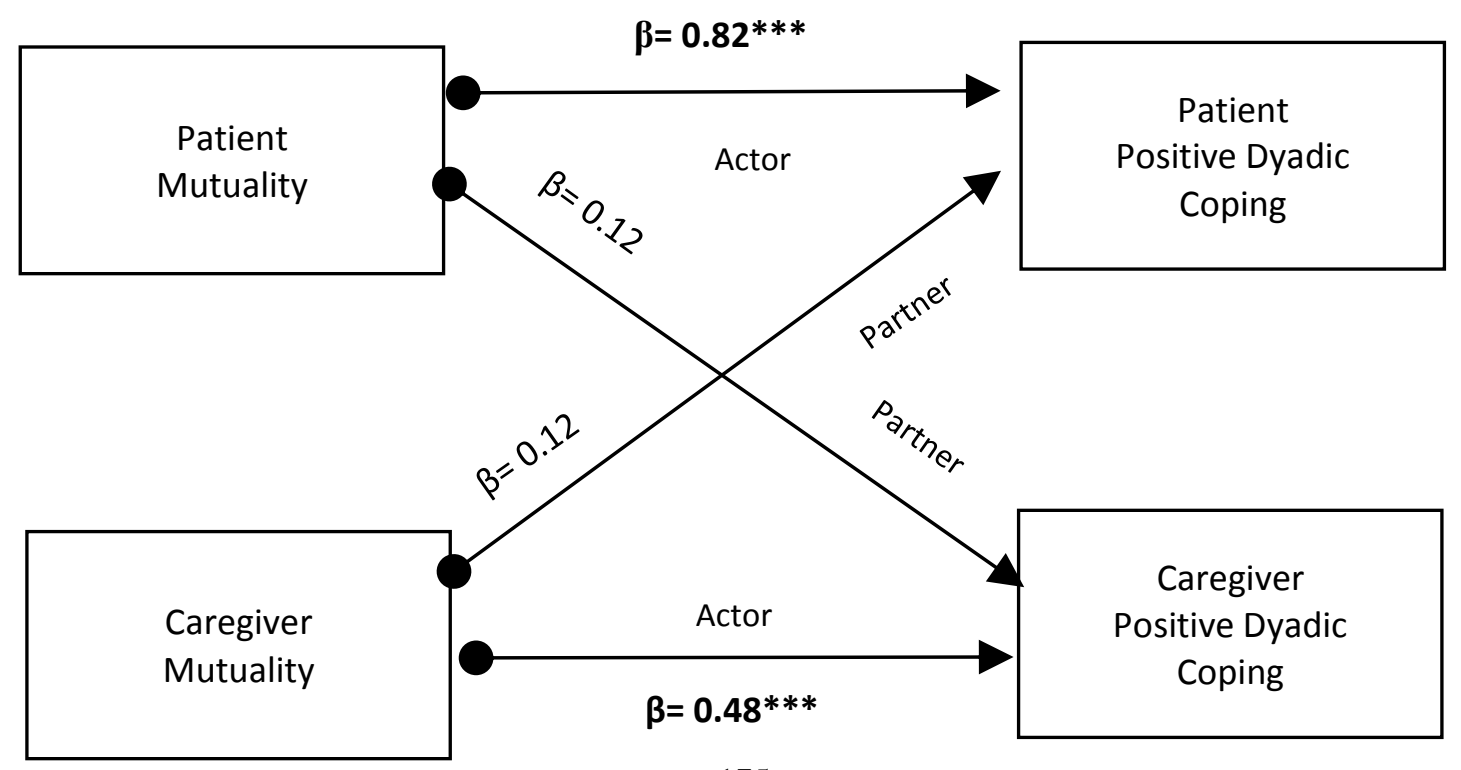




\section{Hostile Dyadic Coping}

To examine actor and partner effects of mutuality on hostile dyadic coping in the two groups, the APIM model was used. The Intraclass Correlation Coefficient indicates that actor and partner's scores on this type of dyadic coping are not interdependent (Younger Couples: ICC $=.004, p>.05$; Older Couples: ICC $=.20, p>.05$ ). The distinguishability test resulted in a significant chi-square test for the young group (Younger Couples: $\chi^{2}{ }_{(4)}=18.39, p<.01$; Older Couples: $\chi^{2}{ }_{(4)}=3.20, p>.05$ ). However, both were treated as distinguishable in the following analyses.

Results are presented in Tables 41-43. For younger dyads, the analysis revealed the presence of an actor effect of mutuality on hostile dyadic coping, next to a significant interaction of role and partner effect. Younger individuals reporting high levels of mutuality were less likely to report hostile dyadic coping. Specifically, a one unit increase in the mean score of mutuality is associated to a 0.84 unit reduction in this negative coping style. To test whether the partner effect is different by patients and partner, a simple slope model was estimated using a two intercept approach (Table 42). In the younger group, the partner effect of mutuality on hostile dyadic coping showed two different directions. For caregivers, the partner effect was not significant. In contrast, for patients, when their caregiver presents higher scores on mutuality, a .50 unit increase in hostile dyadic coping occurs. Among younger dyads both the average partner effect of mutuality and the interaction role by actor mutuality approached significance, and this was possibly due the limited sample size $(p=.058 ; p=.061)$.

Evidence exists for both an average actor and partner effect among older couples. Patients and caregivers presenting higher levels of mutuality, were more likely to report 
lower levels of hostile dyadic coping. Similarly, having a partner reporting high scores on mutuality was associated with reduced hostile coping. Specifically, a one unit increase in mutuality was associated to a 0.23 unit reduction in the hostile dyadic coping score, while a one unit increase in the mutuality score of the partner produces a 0.16 reduction in hostile dyadic coping of the individual. No evidence for differences by role was identified for older dyads (Table 43).

Finally, the approximate proportion of variance in hostile dyadic coping explained by actor and partner mutuality was estimated. For younger patients and caregivers pseudo $\mathrm{R}^{2}$ were .59 and .52 respectively, indicating that a very large proportion of variance was explained by mutuality. In the older counterpart, pseudo $\mathrm{R}^{2}$ for patients was 0.18 and the $\mathrm{R}^{2}$ for caregivers was 0.27 , suggesting that mutuality is particularly relevant for hostile dyadic coping among the younger group.

Table 41. Multilevel regression coefficients estimating Actor and Partner effects of Mutuality on Hostile Dyadic Coping in Younger Couples

\begin{tabular}{lccc}
\hline & $b$ & $\beta$ & $t(d f)$ \\
\hline Intercept & 2.11 & & \\
Role & -0.01 & -0.02 & $-0.23(32)$ \\
Actor Mutuality & $\mathbf{- 0 . 8 4}$ & $\mathbf{- 0 . 9 6}$ & $\mathbf{- 8 . 5 4 * * * ( 5 2 )}$ \\
Partner Mutuality & 0.23 & 0.26 & $1.95(39)$ \\
Role*Actor Mutuality & -0.18 & -0.21 & $-1.91(58)$ \\
Role*Partner Mutuality & $\mathbf{0 . 2 7}$ & $\mathbf{0 . 3 0}$ & $\mathbf{2 . 3 2} *(\mathbf{4 0})$ \\
\hline
\end{tabular}

Note. Participant role was coded patient $=1$, caregiver $=-1$.

$* p<.05, * * p<.01, * * * p<.001$ 
Table 42. Simple slope regression coefficients estimating separate mutuality actor and partner effects for patients and caregivers on Hostile Dyadic Coping in Younger Couples

\begin{tabular}{lccc}
\hline & $b$ & $\beta$ & $t(d f)$ \\
\hline Intercepts & & & \\
Patients & 2.09 & & \\
Caregivers & 2.13 & & \\
Actor's Mutuality & & & $-6.87 * * *(32)$ \\
Patients & -1.02 & -1.16 & $-5.35^{* * *}(32)$ \\
Partners & -0.66 & -0.75 & \\
Partner's Mutuality & & & $\mathbf{2 . 2 9} *(\mathbf{3 2})$ \\
Patients & $\mathbf{0 . 5 0}$ & $\mathbf{0 . 5 6}$ & $\mathbf{- 0 . 4 3 ( 3 2 )}$ \\
Caregivers & $\mathbf{- 0 . 0 4}$ & $\mathbf{- 0 . 0 4}$ & \\
\hline
\end{tabular}

${ }^{*} p<.05, * * p<.01, * * * p<.001$

Figure 17. The actor and partner effects of Mutuality as predictors of Hostile Dyadic Coping in Younger Couples. APIM with distinguishable dyads regression model. $* \mathrm{p}<.05, * * \mathrm{p}<.01, * * * \mathrm{p}<.001$

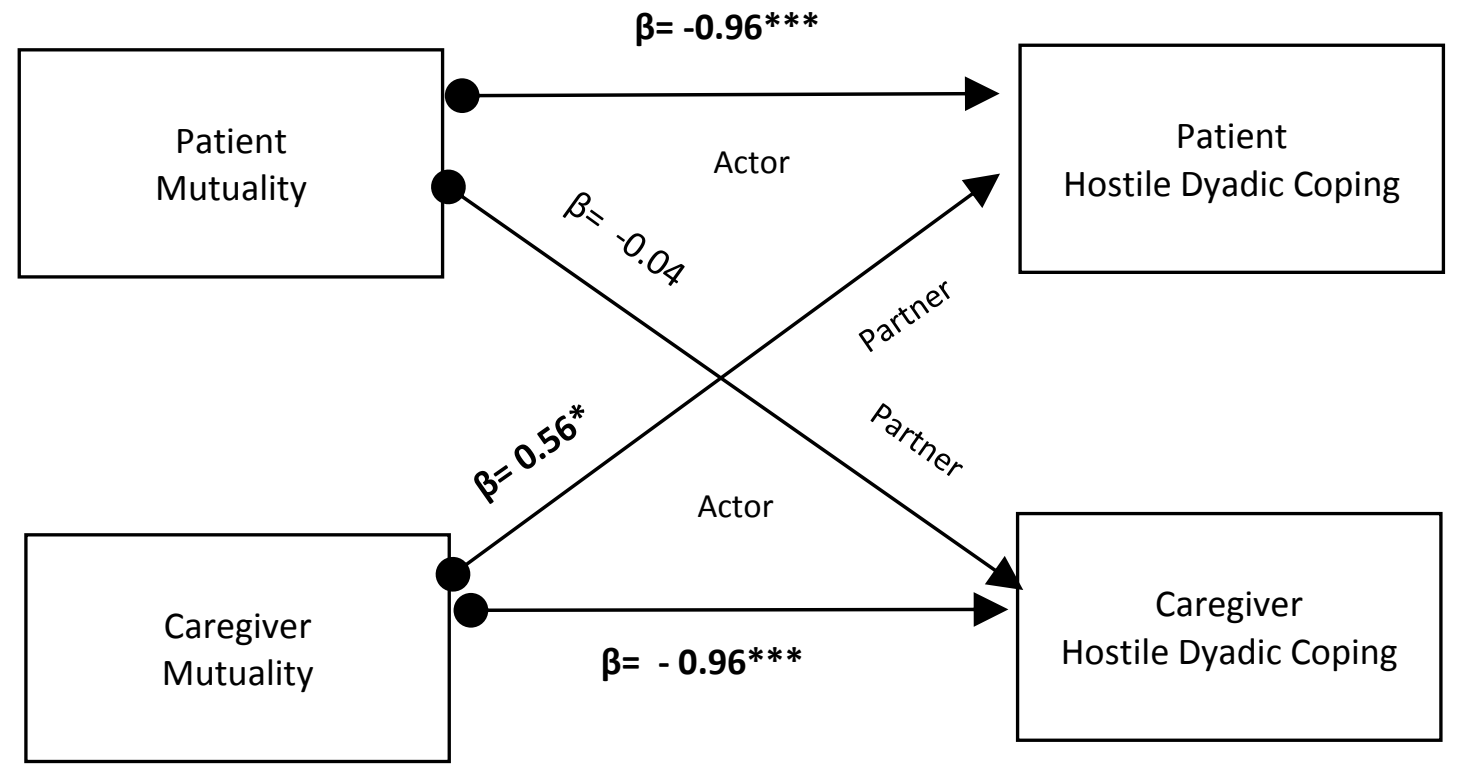


Table 43. Multilevel regression coefficients estimating actor and partner effects of Mutuality on Hostile Dyadic Coping in Older Couples

\begin{tabular}{lccc} 
& $b$ & $\beta$ & $t(d f)$ \\
\hline Intercept & \multicolumn{1}{c}{$\beta$} & & \\
Role & 0.03 & 0.04 & $0.55(48)$ \\
Actor Mutuality & $\mathbf{- 0 . 2 3}$ & $\mathbf{- 0 . 2 6}$ & $\mathbf{- 3 . 7 3} * * *(\mathbf{8 4})$ \\
Partner Mutuality & $\mathbf{- 0 . 1 6}$ & $\mathbf{- 0 . 1 8}$ & $\mathbf{- 2 . 5 5 * ( 8 6 )}$ \\
Role*Actor Mutuality & -0.05 & -0.06 & $-0.80(84)$ \\
Role*Partner Mutuality & 0.10 & 0.11 & $1.57(87)$ \\
\hline Ne. Part
\end{tabular}

Note. Participant role was coded patient $=1$, caregiver $=-1$.

$* p<.05, * * p<.01, * * * p<.001$

Figure 18. The actor and partner effects of Mutuality as predictors of Hostile Dyadic Coping in Older Couples. APIM with distinguishable dyads regression model.

$* p<.05, * * p<.01, * * * p<.001$

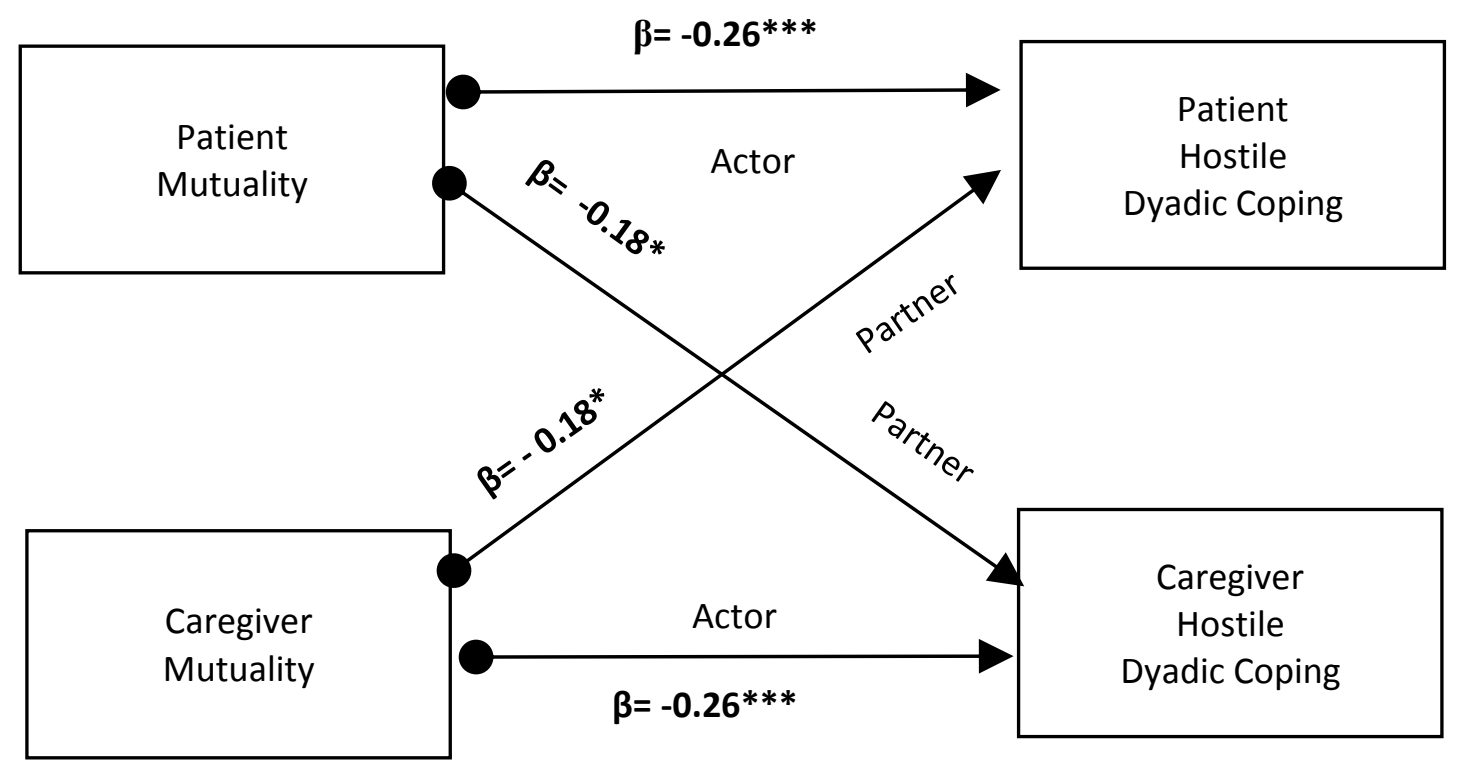




\section{Avoidance of Dyadic Coping}

Finally, the last analysis examined avoidance of dyadic coping in the two groups. The Intraclass Correlation Coefficient indicates that actor and partner's scores on this type of dyadic coping are not interdependent in both groups (Younger Couples: ICC $=.09, p>.05$; Older Couples: $\mathrm{ICC}=.26, p>.05)$. It was then tested whether role should be treated as a distinguishable factor in the analyses. The distinguishability test was significant for the younger group $\left(\chi^{2}(4)=11.786, p<.05\right)$, while the results failed to reach significance for older participants (Appendix B). Despite this, older dyads were treated as distinguishable.

The results of the APIM analysis are presented in Table 44 and 45. Among younger couples, only an average actor effect was detected. The absence of other significant results suggests that no differences are associated to the role covered by the individual in the couple or that the mutuality scores reported by the other member of the dyad do not predict avoidant dyadic coping behaviors. Present findings indicate that as mutuality increases, so avoidance of dyadic coping is reduced by .90 points in younger participants. Differently, for older couples both an average actor and partner effect of mutuality exist. This indicates that there are no differences in the actor and partner effects of patients and caregivers. A one unit increase in the mean score of mutuality is associated to .40 reduction in avoidance of dyadic coping among older patients and caregivers. Having a partner high on mutuality, contributed to reduced maladaptive coping. Among older participants, patients and caregivers experience a .22 reduction in avoidance of dyadic coping for every unit increase in mutuality of their partner. 
Finally, the approximate proportion of variance in avoidance of dyadic coping explained by actor and partner mutuality was estimated. For younger patients and caregivers pseudo $\mathrm{R}^{2}$ were .29 and .53 respectively, indicating a larger impact of mutuality for the younger caregivers' outcome score. In the older group, pseudo $\mathrm{R}^{2}$ for patients was 0.22 and the $\mathrm{R}^{2}$ for partners was 0.18 , indicative of the fact that approximately $22 \%$ and $18 \%$ of the variance in avoidance of dyadic is accounted for by patients and partners' mutuality.

Table 44. Multilevel regression coefficients estimating Actor and Partner effects of Mutuality on Avoidance of Dyadic Coping in Younger Couples

\begin{tabular}{lrrc}
\hline & $b$ & \multicolumn{1}{c}{$\beta$} & $t(d f)$ \\
\hline Intercept & 2.74 & & \\
Role & 0.06 & 0.07 & $0.69(32)$ \\
Actor Mutuality & $\mathbf{- 0 . 9 0}$ & $\mathbf{- 0 . 7 2}$ & $\mathbf{- 5 . 8 3 * * * ( 5 3 )}$ \\
Partner Mutuality & 0.01 & 0.01 & $0.08(40)$ \\
Role*Actor Mutuality & 0.08 & 0.06 & $0.53(59)$ \\
Role*Partner Mutuality & 0.25 & 0.20 & $1.38(41)$ \\
\hline
\end{tabular}

Note. Participant role was coded patient $=1$, caregiver $=-1$.

$* p<.05, * * \mathrm{p}<.01, * * * \mathrm{p}<.001$

Figure 19. The actor and partner effects of Mutuality as predictors of Avoidance of Dyadic Coping in Younger Couples. APIM with distinguishable dyads regression model. $* p<.05, * * p<.01, * * * p<.001$

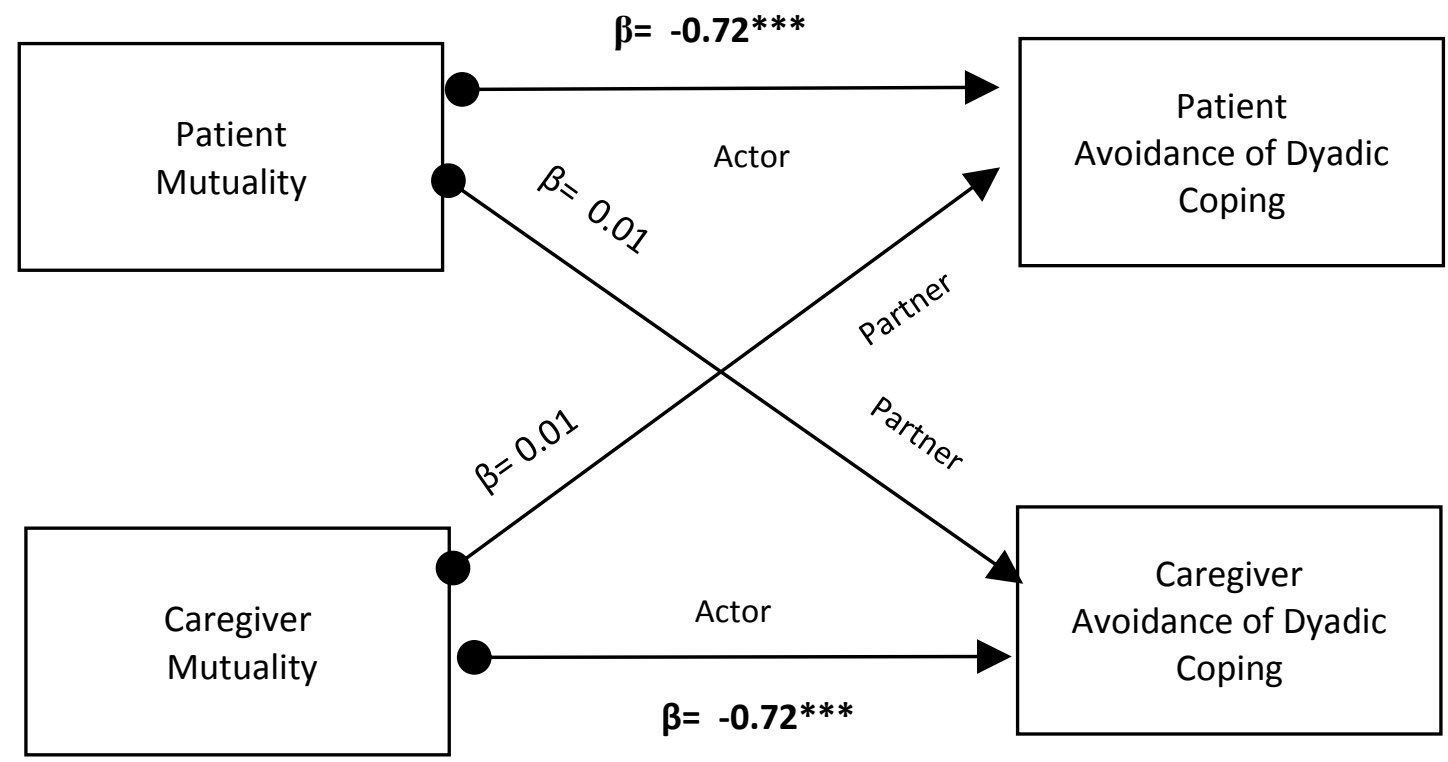


Table 45. Multilevel regression coefficients estimating actor and partner effects of Mutuality on Avoidance of Dyadic Coping in Older Couples

\begin{tabular}{lccc}
\hline & $b$ & $\beta$ & $t(d f)$ \\
\hline Intercept & 2.57 & & \\
Role & 0.04 & 0.05 & $0.74(48)$ \\
Actor Mutuality & $\mathbf{- 0 . 4 0}$ & $\mathbf{- 0 . 3 2}$ & $\mathbf{- 3 . 7 5 * * * ( 8 0 )}$ \\
Partner Mutuality & $\mathbf{- 0 . 2 2}$ & $\mathbf{- 0 . 1 8}$ & $\mathbf{- 2 . 1 4 * ( 9 2 )}$ \\
Role*Actor Mutuality & -0.13 & -0.10 & $-1.21(74)$ \\
Role*Partner Mutuality & 0.02 & 0.01 & $0.21(83)$ \\
\hline
\end{tabular}

Note. Participant role was coded patient $=1$, caregiver $=-1$

$* p<.05, * * \mathrm{p}<.01, * * * \mathrm{p}<.001$

Figure 20. The actor and partner effects of Mutuality as predictors of Avoidance of Dyadic Coping in Older Couples. APIM with distinguishable dyads regression model. $* p<.05, * * p<.01, * * * p<.001$

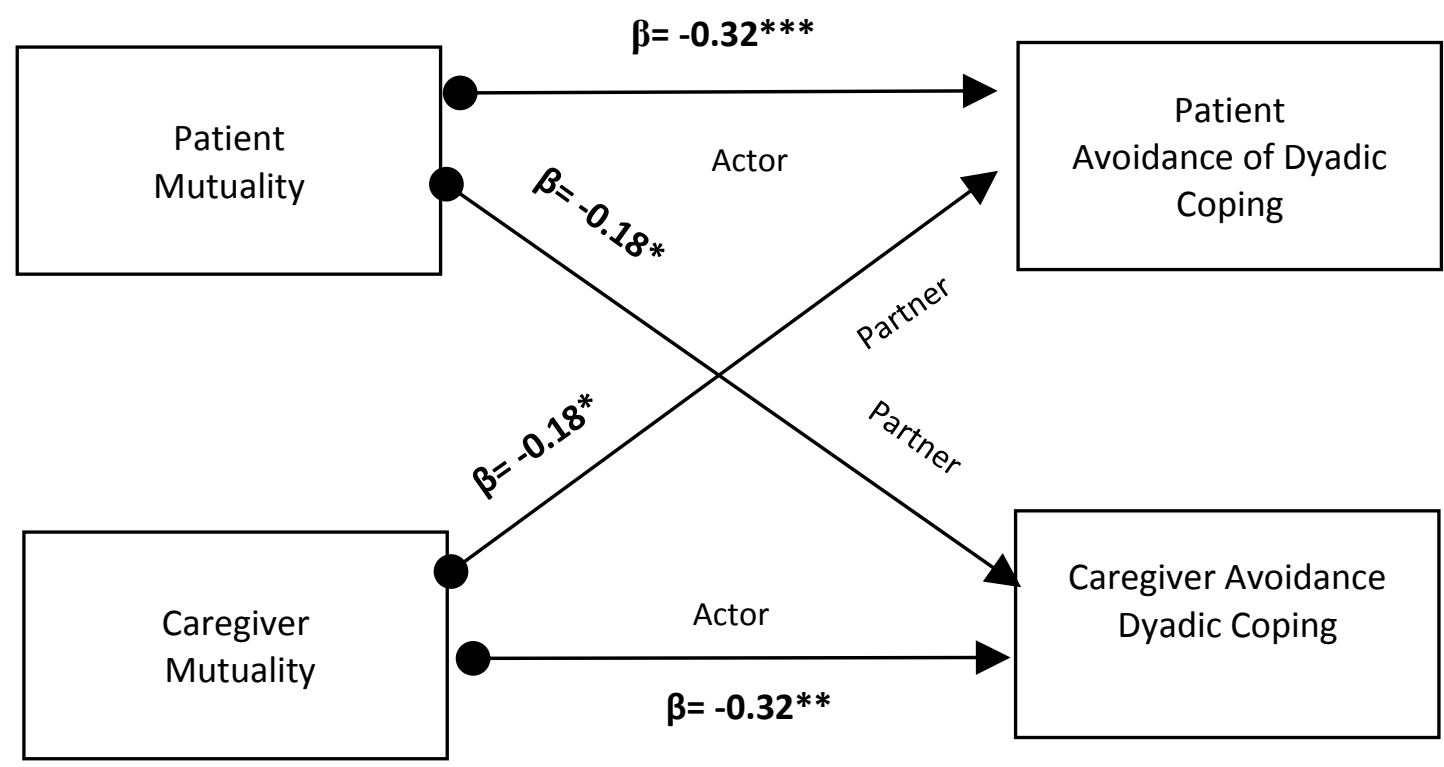


Specific Aim 4: To test the mediating role of dyadic coping on the relationship between Mutuality and Quality of Life of patients and partners and to identify differences by age group

Hypothesis 4.1: Self-reported dyadic coping score will mediate the relationship between mutuality and quality of life of breast cancer patients

Hypothesis 4.2: Self-reported dyadic coping score will mediate the relationship between mutuality and quality of life of partners of breast cancer patients Hypothesis 4.3: Differences exist by age group, between younger and older dyads

Finally, the present study was aimed at investigating the relationship between mutuality, dyadic coping and quality of life to further our understanding of how adaptive relational coping style contributes to better individual quality of life and higher satisfaction, as evidenced by the literature. In particular, by using the Relational-Cultural Model of dyadic coping (Kayser et al., 2007) as the guiding framework, it was hypothesized that dyadic coping would mediate the relationship between mutuality and quality of life for both patients and partners. More simply, it was anticipated that higher scores on mutuality would have contributed to the individual's engagement in adaptive dyadic coping styles, which would have ultimately predicted higher quality of life and reduced the impact of the illness' demands.

Regression analysis was initially used to investigate the hypothesis that dyadic coping mediates the effect of mutuality on quality of life of cancer patients and caregivers, controlling for age. This variable was preferred to duration of relationship because of the high positive correlation between the two variables $(r=.66, p<.001)$, especially for females $(r=.75, p<.001)$. Models were run for each dyadic coping style and each subscale and total score on the FACT-B for breast cancer patients, while for partners the tested models included all the dyadic coping styles and the two variables of 
emotional well-being and illness intrusiveness. No significant result emerged from this first approach and also removal of age did not contribute to the identification of significant mediation models for patients and partners.

Given the overall aim of the present study it was then decided to divide each group by age and to run the analysis separately for younger and older patients and partners using the following equations: ${ }^{36}$

$$
\begin{gathered}
M=i_{1}+a X+e_{M} \\
Y=i_{2}+c^{\prime} X+b M+e_{Y}
\end{gathered}
$$

where $i$ are the regression intercepts, $e$ are the errors in the estimation of $\mathrm{M}$ and $\mathrm{Y}$, and $a, b$, and $c$ ' represent the path between $\mathrm{X}$ and $\mathrm{M}, b$ the path between $\mathrm{M}$ and $\mathrm{Y}$, and $c$ ' the direct effect of $\mathrm{X}$ on $\mathrm{Y}$ (see Figure 21). The product of $a$ and $b$ is the indirect effect of $\mathrm{X}$ on $\mathrm{Y}$ through $\mathrm{M}$ and indicates how much two cases that differ by one unit on the predictor $(\mathrm{X})$ are estimated to differ on the outcome in $a b$ units as a result of the effect of the predictor on the mediator $(\mathrm{M})$, which ultimately affects the score reported on the outcome (Y). Of the 84 models tested for patients and the 24 models analyzed for partners, only two led to significant results and are presented in the following paragraphs.

\footnotetext{
${ }^{36}$ A mediation analysis was initially performed using the Baron and Kenny casual steps approach (1986) to identify that significant relationships exist among the variables included in the analysis. However given the limitations of the approach and the emergence in recent years of more accurate modalities to test the significance of the indirect effect the PROCESS macro developed by Hayes (2013) was used and a bootstrap estimation approach implemented
} 
Figure 21. Statistical diagram of the Simple Mediation Model

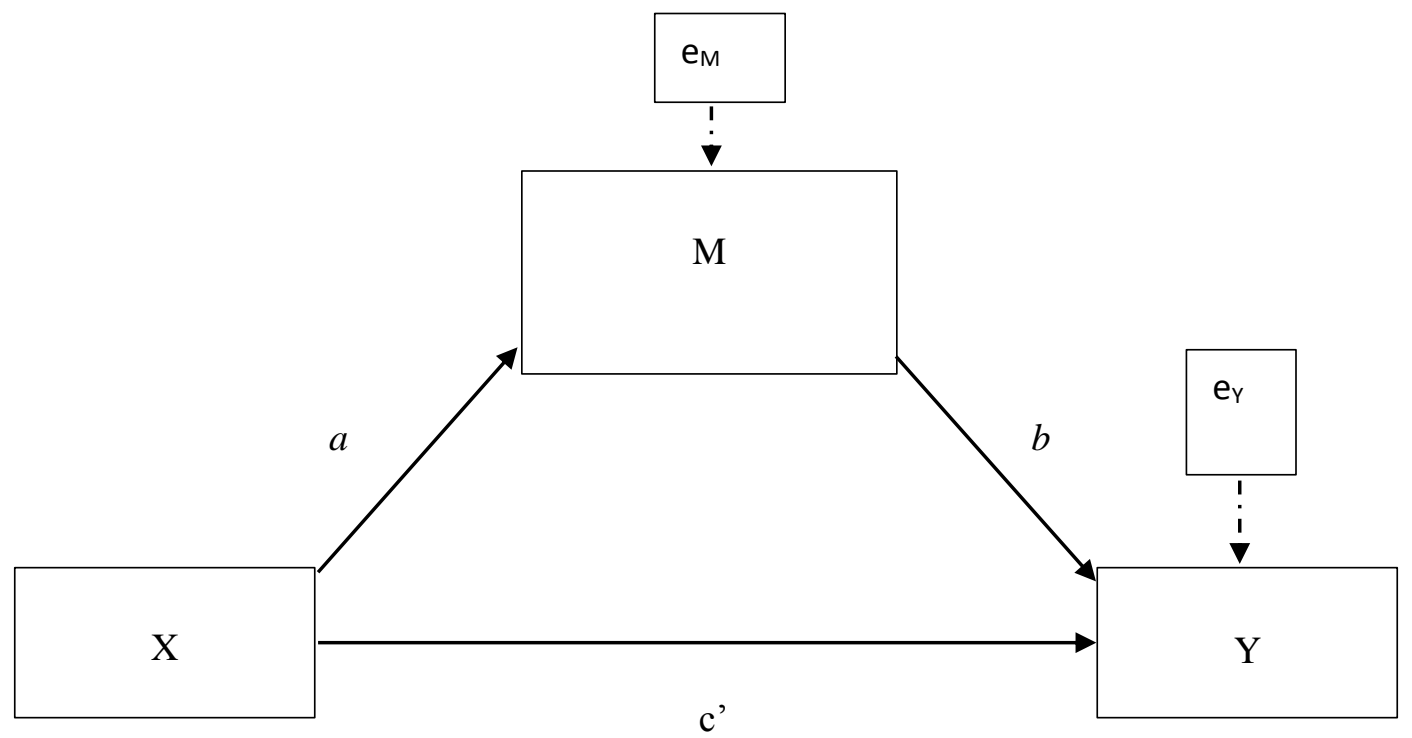

Regression analysis was used to investigate the hypothesis that avoidance of dyadic coping mediates the relationship between mutuality and social well-being for younger women with breast cancer (Table 46). Results indicate that mutuality was a significant predictor of avoidance of dyadic coping $(\mathrm{b}=-.516, \mathrm{SE}=.203, p<.05)$ and that avoidance of dyadic coping was a significant predictor of social well-being score $(b=-.1 .323, \mathrm{SE}=$ $.580, p<.05)$. These results support the mediational hypothesis. After controlling for the mediator, mutuality was still a significant predictor of social well-being ( $b=3.406$, $\mathrm{SE}=.580, p<.05)$; which is consistent with a partial mediation. Approximately $57 \%$ of the variance in younger women social well-being was accounted for by the predictors $\left(\mathrm{R}^{2}=\right.$ .567). The significance of the indirect effect was tested using a bootstrap estimation approach with 5000 samples (Hayes, 2013; Shrout \& Bolger, 2002). Results indicate that the indirect coefficient was significant $(b=.682, \mathrm{SE}=.509,95 \% \mathrm{CI}=.062,2.167)$. If we 
illustrate these relationships in a statistical diagram, we obtain the following figure (Figure 22).

Figure 22. Simple Mediation Model for Younger Patients

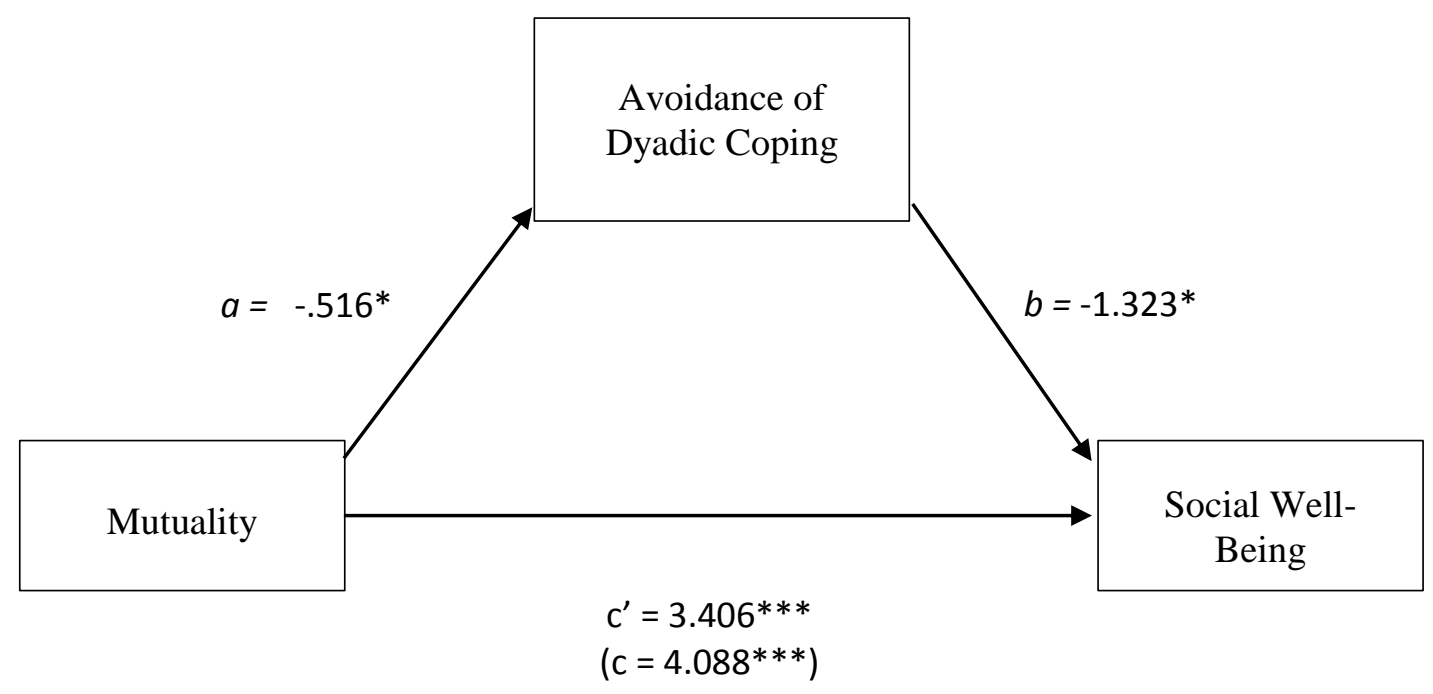

Present findings indicate that for each unit increase in mutuality, younger patients are estimated to report a .516 reduction in the score on avoidance of dyadic coping. Younger women who differ by one unit on avoidance of dyadic coping are expected to differ by 1.323 units on social well-being, with women higher on avoidance of dyadic coping reporting lower scores on social well-being. The total effect $c$ indicates how much two cases that differ by one unit on mutuality are estimated to differ in social well-being. In our sample one unit increase on mutuality contributes to a 4.09 unit increase in social well-being, while this increase is limited to 0.68 as the result of the effect of mutuality on avoidance of dyadic coping ( $a b$, indirect effect). The direct effect of mutuality on social well-being indicates that women who report the same score on avoidance of dyadic Coping but who differ by one unit in mutuality are estimated to report a 3.41 increase in 
social well-being. Practically speaking, for younger women the positive effect of mutuality on their social well-being is negatively impacted by avoidance of dyadic coping, which significantly reduces their quality of life. 
Table 46. Model coefficients of the Simple Mediation Model between Mutuality, Avoidance of Dyadic Coping and Social Well-being for Younger Patients

\begin{tabular}{|c|c|c|c|c|c|c|c|c|}
\hline \multirow[b]{3}{*}{ Antecedent } & & \multicolumn{4}{|c|}{ Consequent } & \multirow{2}{*}{\multicolumn{3}{|c|}{ Y (Social Well-being) }} \\
\hline & & \multicolumn{3}{|c|}{ M (Avoidance of Dyadic Coping) } & & & & \\
\hline & & Coeff & SE & $p$ & & Coeff & SE & $p$ \\
\hline X(Mutuality) & $a$ & -.516 & .203 & .016 & $c^{\prime}$ & 3.406 & .738 & $<.001$ \\
\hline M (Avoidance of & & - & - & - & $b$ & -1.323 & .580 & .020 \\
\hline Constant & $\mathrm{i}_{1}$ & 5.053 & .899 & $<.001$ & $i_{2}$ & 10.304 & 4.187 & .019 \\
\hline & & \multicolumn{3}{|c|}{$\begin{array}{l}\mathrm{R}^{2}=0.164 \\
\mathrm{~F}(1,33)=6.476, p<.05\end{array}$} & & \multicolumn{2}{|c|}{$\begin{array}{l}\mathrm{R}^{2}=.567 \\
\mathrm{~F}(2,32)=20.947, p<.001\end{array}$} & \\
\hline
\end{tabular}


The second significant simple mediation model was identified for older partners. Regression analysis was used to investigate the hypothesis that hostile dyadic coping mediates the relationship between mutuality and illness intrusiveness (Table 47). Results indicate that mutuality was a significant predictor of hostile dyadic coping $(b=-.415, \mathrm{SE}=$ $.107, \mathrm{p}<.001)$ and that hostile dyadic coping was a significant predictor of illness intrusiveness $(b=9.618, \mathrm{SE}=4.317, \mathrm{p}<.05)$. These results support the mediational hypothesis. After controlling for the mediator, mutuality was no longer a significant predictor of illness intrusiveness $(b=-3.70, S E=3.69, p>.05)$, which is consistent with full mediation. Approximately $18 \%$ of the variance in older partners' impact of the patients' illness on their quality of life was accounted for by the predictors $\left(\mathrm{R}^{2}=.181\right)$. The significance of the indirect effect was tested using a bootstrap estimation approach with 5000 samples (Hayes, 2013; Shrout \& Bolger, 2002). Results indicate that the indirect coefficient was significant $(b=-3.996, \mathrm{SE}=2.585,95 \% \mathrm{CI}=-10.159 ;-.032)$. 
Table 47. Model coefficients of the Simple Mediation Model between Mutuality, Hostile Dyadic Coping and Illness Intrusiveness for Older Partners

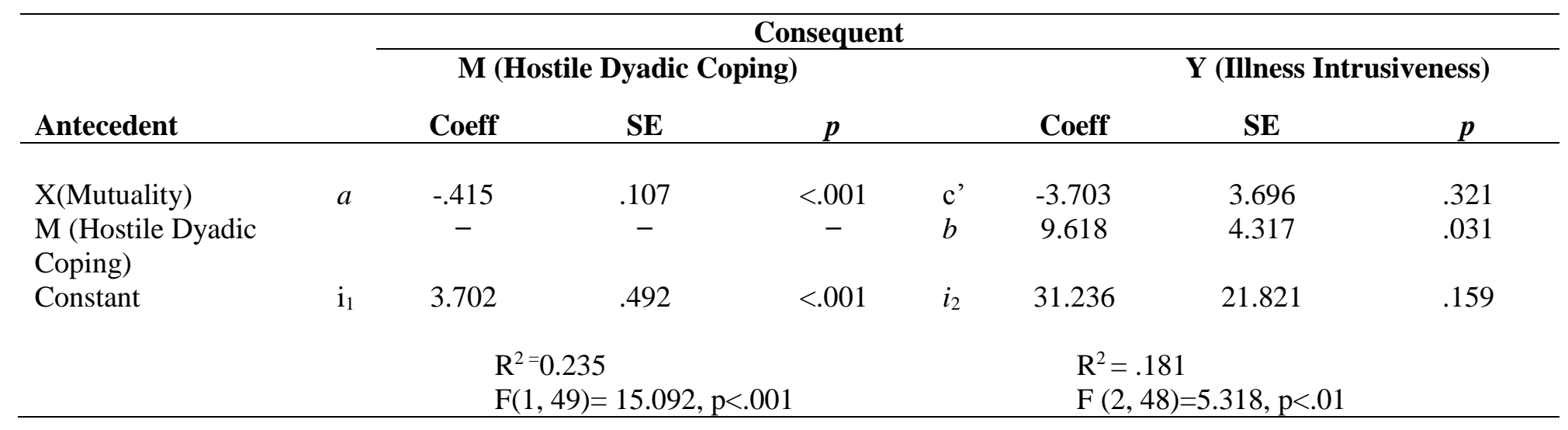


Figure 23. Simple Mediation Model for Older Partners

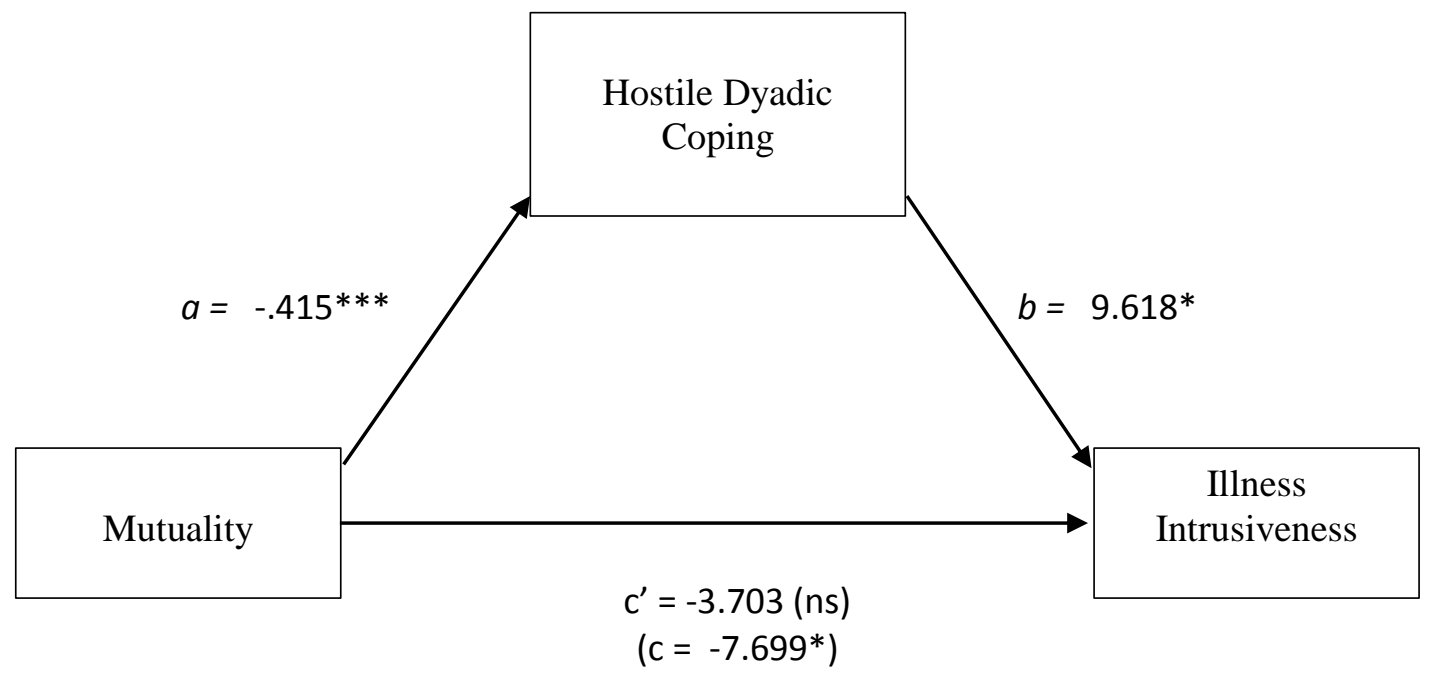

Figure 23 summarizes in a statistical diagram these relationships. Among older partners for each unit increase in mutuality, a .415 reduction in hostile dyadic coping occurs. Caregivers who differ by one unit on hostile dyadic coping are expected to differ by 9.618 units on illness intrusiveness, with higher impact of the illness demands on the partner's quality of life for those higher on hostile dyadic coping. The total effect $c$ indicates how much two cases that differ by one unit on mutuality are estimated to differ in illness intrusiveness. A significant large reduction in the score for illness intrusiveness is in fact estimated $(-7,70)$, while this reduction is equal to 3.966 for the indirect effect of mutuality on hostile dyadic coping ( $a b$, indirect effect). The direct effect of mutuality on illness intrusiveness indicates that older partners who report the same score on hostile dyadic coping but who differ by one unit in mutuality are estimated to report a 3.70 decrease in the outcome measure. Practically speaking, for older partners the protective effect of mutuality on their quality of life, as evidenced by a significant reduction in the score for illness intrusiveness, is negatively impacted by hostile dyadic coping. 


\section{Summary}

The chapter described the results of this investigation of dyads coping with earlystage breast cancer. The first aim of the study was to identify differences between younger and older couples on quality of life, mutuality, communication, and dyadic coping. As hypothesized, younger women with breast cancer reported worse quality of life compared to older patients. Specifically, younger women experienced more affected physical, social and emotional functioning, when compared to women diagnosed with breast cancer at older age. However, no statistically significance difference existed in the present sample for mutuality, communication and dyadic coping styles, suggesting that younger and older women have similar abilities in communicating with a partner, in the repertoire of dyadic coping strategies, as well as in their self-reported level of mutuality. Hence this hypothesis can only be partially accepted. The younger group of partners did report higher scores of maladaptive dyadic coping, lower mean scores of stress communication, common and positive dyadic coping, and worse quality of life than the older group. However, significant mean differences were identified only in hostile dyadic coping, emotional well-being, and illness intrusivenesss suggesting that also this hypothesis was only partially supported by the results. Differences between younger and older couples were also investigated referring to the diverse amount of association of qualify of life scores and dyadic coping scores between younger and older patients and partners. Although it was hypothesized that associations between older patients and partners would have been higher, both these hypotheses are rejected in their current forms. Correlations coefficients of younger couples were higher for quality of life and common positive dyadic coping. However, results of this analysis suggest that a further 
reflection is necessary. First, the association between patients and partners' quality of life in older couples exists for both measures of quality of life, while among the younger group significant correlations are limited to emotional well-being. Second, if the association between dyadic coping scores are considered, in the older group patients and partners' association of dyadic coping scores extended also to negative coping styles, while this was not reported in the younger dyads.

The relationship between dyadic coping and quality of life for young breast cancer patients and partners was then considered. While for younger women with breast cancer positive forms of dyadic coping are associated with higher levels of quality of life, especially for their social well-being, surprisingly no association was found for their partners. Hence, the hypothesized association between positive dyadic coping styles and quality of life is accepted only for patients. The next step of the analysis was to test whether the relationship between dyadic coping styles and quality of life for patients and partners is influenced by socio-demographic, clinical, relational and mental health variables. The limited sample size of the younger group required for the multiple regression to be conducted on the whole sample of patients and partners. Multiple regression analyses unveiled the role age and hostile dyadic coping have for the quality of life of both patients and partners, with these two variables consistently identified among the predictors of worse quality of life scores in the present sample.

The third aim of the study was to assess the actor and partner effects of mutuality on dyadic coping among breast cancer patients and partners, and to identify differences by age group. As hypothesized, patients' and partners' perceived mutuality influenced their own and their partner's dyadic coping but only for a limited number of dyadic 
coping style. Findings from the initial analysis indicate that both patients and caregivers' scores on mutuality contribute to common, positive and reduced avoidance of dyadic coping. More complex was the scenario for hostile dyadic coping. In the overall sample, patients and caregivers' mutuality predicted a reduction in their own hostile dyadic coping, but having a partner high on mutuality reduced these coping behaviors only for caregivers. It was then the decision to conduct separate APIM analyses on younger and older dyads that allowed for the identification of different relational exchanges in the two groups, which would have been otherwise unnoticed. Several differences in common, positive, hostile and avoidance of dyadic coping were identified between younger and older couples, which will be discussed in detail in the next chapter.

Finally, the mediating role of dyadic coping on the relationship between mutuality and quality of life of patients and partners was examined. Only two models were significant, with avoidance of dyadic coping mediating the relationship between mutuality and social well-being for younger women with cancer. The other significant result was identified for older partners. For them the relationship between mutuality and illness intrusiveness is mediated by hostile dyadic coping. It follows that the three hypotheses identified for this last aim of the study can only be partially accepted and future investigation is needed. In the next chapter the relevance of these findings will be discussed, specifically by addressing the implications for future research and practice. 


\section{CHAPTER V: DISCUSSION}

The final chapter of the dissertation presents the implications of the results described in

the prior chapter. First, the findings of the performed analyses will be discussed in relation to whether they converge or diverge with the existing literature. Next, implications for practice will be outlined. Finally, study limitations and future directions of research about younger couples coping with cancer will be delineated.

In the last decade there has been an increasing recognition that younger women with breast cancer represent a separate group among all women diagnosed with this illness because of unique clinical and psychosocial issues. Among the most relevant problems reported by younger women, there is an increased concern for their relationship with the partner. However, despite the recent trend toward a relational view of cancer and the evidence that has identified more difficulties and challenges for young couples, the literature hasn't been attentive and responsive to this topic. There are only a limited number of studies addressing younger women's close relationships or including both partners. The present study has addressed this gap investigating younger couples' adaptation and coping with the diagnosis of breast cancer, and by comparing them to a group of older dyads. More specifically, given the increasing interest in the psychosocial 
literature about dyadic coping and its impact on marital quality and individual's wellbeing in the context of a chronic illness, the relationship among mutuality, dyadic coping and quality of life of patients and partners has been examined to test for empirical evidence a conceptual framework inspired by the Relational-Cultural Model of dyadic coping (Kayser et al., 2007).

\section{Discussion}

Results of this cross-sectional investigation confirm the differential impact a diagnosis of cancer has on younger patients and partners' quality of life. Compared to older couples, younger patients and their partners' adjustment to cancer is significantly compromised within the first three months from diagnosis, with impaired functioning and significant worse quality of life. Younger women in this sample experienced a more negative impact of the illness such as elevated side effects of treatment, reduced closeness to friends, low support from family/partners and more difficult adjustment to the illness. Compared to their older counterparts, the younger group also had more numerous symptoms associated with the illness, concerns for their body image and heightened self-consciousness. These findings are consistent, despite the small sample, with results from larger studies targeting the quality of life of younger women with breast cancer (Avis et al., 2004; Avis et al., 2005; Baucom et al., 2005; King et al. 2000; Kroenke et al., 2004; Wenzel et al., 1999; Ganz et al., 2003; Howard-Anderson et al., 2012; Luutonen et al., 2011). In particular, when the same instrument (FACT-B) has been administered to younger patients, other authors have indeed identified the presence of significant differences between younger and older breast cancer patients' overall quality 
of life (Avis et al., 2005; Di Sipio et al., 2008; Brennan et al., 2014), with more negative physical well-being, emotional well-being and breast cancer symptoms that resonate with our results (Park, Lee, Lee, Lee \& Hwang, 2011; So et al, 2011). Furthermore, when compared to normative data (Brucker et al., 2005) ${ }^{37}$, younger women from this study score significantly worse than other female patients with cancer $\left(t_{(34)}=-3.80, p<.01\right)$ and healthy peers $\left(t_{(34)}=-2.78, p<.01\right)$, confirming the unique experience of this group.

In a similar way, younger partners did report a more negative impact of the illness on their quality of life, with statistically significant higher intrusiveness, lower emotional well-being, and a higher use of maladaptive dyadic coping styles compared to partners of older breast cancer patients. While earlier works only postulated increased difficulties for younger partners (Baucom et al., 2005), the results of the present study contribute to the evidence collected by the few studies to date that found a more detrimental effect of the patient diagnosis on the quality of life of younger partners, who are faced especially with concerns about everyday life, negative affectivity, apprehension about the future and the couple cohesion (Antoine et al., 2012; Christophe et al., 2015; Duggleby et al.,2014; Fergus et al., 2015; Hasson-Ohayon et al., 2014; Vanlemmens et al., 2015a; Vanlemmens et al., 2015b). Finally, the higher score on illness intrusiveness is a finding that is similar to results emerged in the literature about cancer caregiving, which has identified higher burden, mood disturbances and worse quality of life for those who assume this role at a

\footnotetext{
${ }^{37}$ General population and cancer patient norms for the Functional Assessment of Cancer Therapy - General (FACT-G; Cella, 2004) have been collected for Physical, Social/Family, Emotional and Functional Wellbeing and the FACT General score. A one-sample t test analysis was conducted comparing younger and older patients' score on the FACT-G with the data of the general U.S. adult population and the normative data of cancer sample for females.
} 
younger age (Harden et al., 2013; Kim, Spillers, \& Hall, 2012; Lee et al., 2013; Shahi et al., 2014; Sjolander, Rolander, Järhult, Martensson \& Ahlstrom, 2012).

While these results confirmed the greater negative impact of a diagnosis of breast cancer on the two individuals, little is known about younger couples' coping abilities and their impact on quality of life because previous studies on younger breast cancer patients and partners did not examine dyadic coping. While it was hypothesized that quality of life and dyadic coping scores were less correlated within the younger group, the examination of these relationships suggests that younger couples are characterized by moderate to high associations of these constructs. Hence, younger dyads in our sample are characterized by higher reciprocal influence of their quality of life and adaptation to stress. This finding is consistent with the work of some authors who have hypothesized that young couples tend to adhere to a cultural influence that emphasizes similarity and underestimates differences in the early stage of a relationship (Acitelli, Douvan \& Veroff, 1993; Bertoni et al., 2007; Iafrate et al., 2012), and the close relationships literature, which identifies the early years of marriage as a crucial time for the future quality of the relationship (Bradbury \& Karney, 2014, Carra' Mittini, 1999; Lavner \& Bradbury, 2010; Lavner et al., 2013; Scabini \& Cigoli, 2000; Scabini \& Iafrate, 2003; Scabini \& Rossi, 2012; van Steenbergen, Kluwer, \& Karney, 2011). Mature functioning beteween older couples is on the contrary characterized by higher coordination of quality of life and coping mechanisms, with older individuals described in the literature as more willing to accept differences and uniqueness in their couple relationship because of a stronger sense of identity (Berg \& Upchurch, 2007; Bertoni et al., 2007; Blanchard-Fields \& Coats, 2008; Hoppman et al., 2008; Iafrate et al., 2012). This observation can contribute to the 
emergence of a different view about younger and older dyads, where quality may be juxtaposed to quantity. It is not only the amount of association among the members of the dyad to be of interest for the researcher, but also how patients and partners' scores can affect different areas of quality of life and coping across the life trajectory. Hence, it is possible that for younger dyads facing cancer stronger correlations are necessary to support a relationship more vulnerable to stressors, while for mature couples -who have developed more elaborated and coordinated relational exchanges- the role of the other becomes more pervasive on the reciprocal well-being.

In line with these findings are the results related to the second aim of the study, where the relationship between dyadic coping and quality of life was investigated among the younger dyads. While higher quality of life, and specifically higher social well-being, characterized younger women showing positive styles of dyadic coping, the absence of correlation between dyadic coping and quality of life scores of younger partners seems to suggest that for them other variables contributed to their well-being at time of diagnosis, as highlighted by the work of Christophe et al. (2015a; 2015b) about differences between patients and partners. Although unexpected, given the large body of literature that supports the association between dyadic coping and quality of life, this result can also be linked to the different developmental processes the Relational-Cultural Theory identifies for men and women. As men develop through autonomy and separation (Gilligan, 1982; Miller, 1976), it is possible to hypothesize that for younger partners other factors may be relevant to their well-being and that their quality of life may be more dependent on individual coping strategies, personality factors or support received from other contexts rather than on their close relationships. Multiple regression analysis confirmed the role 
younger age and hostile dyadic coping have for the quality of life of both patients and partners, with these two variables consistently identified as predictors of worse quality of life scores. This indication is consistent with the well-established literature that has identified negative dyadic coping strategies as predictors of more affected individual's functioning and adaptation to stress (Falconier et al., 2015; Rottman et al., 2015; Traa et al., 2015b).

Next, the Actor Partner Interdepedence Model furthered our understanding of the underlying dynamics associated with the emergence of different dyadic coping styles within the dyads and between the two groups. While findings from the initial analysis indicate that both patients and caregivers' scores on mutuality contribute to higher adaptive (common, and positive) and reduced negative forms of dyadic coping (avoidance of dyadic coping, and reduced hostile dyadic coping ${ }^{38}$ ), separate APIM models on younger and older dyads unveiled the interaction between patients' and caregivers' mutuality score and how they contribute to different outcomes, especially among the younger group. In constrast to older couples, where actor and partner effects of mutuality predicted reduced hostile and avoidance of dyadic coping, younger couples are characterized by more complex interactions, and both adapative and maladaptive coping results from the patients and partners' perceived mutuality. Most interesting differences between the two groups pertain to common and hostile dyadic coping. Higher scores for common dyadic coping existed for the younger group as a consequence of

\footnotetext{
${ }^{38}$ Hostile dyadic coping is predicted by the individual's mutuality score of both patients and caregivers. Furthermore, role moderated the actor and partner effects of mutuality and results suggest that although both patients and caregivers' own mutuality predicts reduced hostile dyadic coping, the patient's actor effect was significantly stronger than the caregiver's. The partner effect of mutuality was significant only for caregivers, indicating that increases in the mutuality scores of patients were associated to lower levels of hostile dyadic coping in the caregivers.
} 
actor and partner effects, suggesting that higher scores for this coping style are the result of the individual's self-reported scores, as well as the score of the partner. This finding illustrate well the above mentioned higher reciprocal influence of younger couples. The resulting higher adaptive coping predicted by patients and partners' mutuality may represent a great resource to promote couple's adaptation to cancer, because greater relationship satisfaction, dyadic adjustment and less distress have been consistently associated with common dyadic coping (Badr et al., 2010; Bodenmann et al., 2010; Papp \& Witt, 2010; Rottman et al., 2015). However, this interdependence represents an element of risk whether discrepancies in mutuality may exist within partners, or when both partners are not able to engage in this exchange of thoughts, feelings and actions (Jordan, 1997). Similarly, the results obtained for hostile dyadic coping call for a greater attention to the adaption of couples facing cancer earlier in life. Younger patients and caregivers high on mutuality reported lower hostile dyadic coping. However, as the mutuality score of the caregiver increases, for younger women with breast cancer hostile dyadic coping is predicted to significantly increase, suggesting that the origin of maladaptive coping behavior in younger women is influenced by the perceived emotional support in their close relationships.

Hence, some considerations can be made. First, these findings confirm the role of relationship qualities in the enactment of dyadic coping behaviors, thus providing empirical support for the Relational-Cultural Model of Dyadic Coping (Kayser et al., 2007). Often neglected by the literature, mutuality emerges as vital in the theoretical reflection about dyadic coping and the relationship among partners (Godwin, Swank, Vaeth, \& Ostwald, 2013; Lyons, Stewart, Archibald, \& Carter, 2009; Park \& 
Schumacher, 2013; Simeone, Savini, Torino, Vellone, \& Alvaro, 2014). The second consideration is that, despite no significant differences have been found between the dyadic coping scores of younger and older patients and partners (with the exception of hostile dyadic coping), dyadic coping originates differently among the two groups. Both positive and negative dyadic coping behaviors in the younger group result from the scores of both partners on mutuality. It follows that, by assuming a Relational-Cultural Model, it is inaccurate to treat younger and older dyads as a homogeneous group because dyadic coping, albeit not statistically different, emerges from different relational exchanges of emotional support. Having a partner high on mutuality has a detrimental effect for younger patients; a datum that indicates the need for interventions aimed at promoting more beneficial relational exchanges in younger dyads and to enhance communication strategies that facilitate the beneficial disclosure of feelings. In this way partners will learn how to express their participation in more effective ways. It is possible that the communication of emotional involvement of the partner can be perceived by the younger patient as a reminder of the illness and its consequences -including the impact of the illness on the partner's well-being-, and thus originates a defensive reaction to minimize and distancing the emotional experience of stress. At the same time, it is also possible to contemplate that these differences exist because men and women in younger couples diverge in their ability to accurately perceive the behaviors of the other partner. Women are more accurate in detecting partner's negative behaviors and men in perceiving positive ones (Donato \& Parise, 2011). As a consequence, by promoting strategies that facilitate the communication of mutuality in the partner, it may be ultimately possible to protect also the quality of life of the woman. 
Finally, the study tested the mediating role of dyadic coping on the relationship between mutuality and quality of life. Although the absence of significance in most of the models tested can be ascribed to the limited sample included in the present investigation, results indicate that negative dyadic coping styles mediate the relationship between mutuality and quality of life for younger patients and older partners. Among younger women, avoidance of dyadic coping partially mediated the relationship between mutuality and their social well-being. This finding highlights the relevance of perceived exchanges of emotional support within the close relationship with a partner in predicting the social well-being of younger women with cancer, and the negative effect of withdrawal and avoidance in a moment of stress. This finding provides further support to the fact that "women's coping abilities are shaped by and continue to develop in the context of ongoing close relationships" (Kayser, Sormanti \& Strainchamps, 1999, p. 727), and that the presence of forms of coping which threaten these relationships negatively impact younger women's perception of closeness with significant ones. The fact that the mediation model was significant only for younger women strengthen the findings earlier discussed about the major disruption caused by cancer in their social well-being. Furthermore, the absence of significant findings suggests again that other factors become relevant for younger partners. Among older partners, the protective effect of mutuality on illness intrusiveness was negatively affected by the perception that the partner was showing disinterest and was minimizing their emotional experience of stress. It is possible to affirm that older partners may be particularly vulnerable to negative forms of dyadic coping, confirming the literature that places higher value on relationships as age increases, especially for men (Birditt \& Fingerman, 2003; Carr, Freedman, 
Cornman, \& Schwarz, 2014; Carstensen, Fung, \& Charles, 2003; Charles \& Piazza, 2007; Fingerman, Hay \& Birditt, 2004; Gurung, Taylor \& Seeman, 2003; Luong, Charles \& Fingerman, 2011)

Summarizing, the present study contributes to the understanding of the experience of younger couples coping with breast cancer. This study has highlighted the more negative effect of the illness on the quality of life of the two partners. It has also examined factors that contribute to this greater vulnerability investigating the different association between dyadic coping and quality of life among the dyads. Younger couples may be more at risk because of the greater association of their respective quality of life and dyadic coping scores, a finding that suggests the need to further understand how dyadic coping develops in this groups and its association with individual and relational outcomes. From this investigation it emerges that patients and partners' mutuality contributes to dyadic coping and that the relationship between mutuality and quality of life is mediated by negative coping styles for younger women and older partners. In particular, among younger couples it appears that both positive and maladaptive outcomes in terms of couple's coping are the results of patients' and partners' mutuality. It therefore follows that both members of the dyad have an essential role in developing coping strategies that will contribute to better adjustment to the disease and the preservation of their relationship. Hence, the study is consistent with the recent trend to identify the mechanism through which dyadic coping originates and leads to individual and relational outcomes (Levesque, Lafontaine, Caron, Flesh \& Bjornson, 2014; Regan et al., 2015; Traa et al. 2015b; Vedes et al., 2013). 


\section{Implications for Practice}

Findings from the present study suggest the need for a renewed attention to the psychosocial issues of patients and partners (Institute of Medicine, 2008; 2013). Social workers and health care professionals need to develop greater understanding of how to work effectively with younger patients and their significant ones, and to promote their ability to find support within the health care system.

First, this study emphasizes the need to address the multiple domains of quality of life that are impacted by a diagnosis of breast cancer at a younger age. As identified by the literature, areas like emotional and social well-being acquire more relevance for younger women. Younger patients have also greater psychosocial needs about the gynecological, sexual and reproductive consequences of treatment (Brennan et al., 2014; Duffy, Allen, \& Clark, 2005; Easley, Miedema \& Robinson, 2013; Howard-Anderson et al., 2012; Gorman et al., 2011). Unfortunately, many of them are not fully aware of the adverse effects of cancer and their informational needs are currently not being met (Avis et al., 2004; Gorman et al, 2011; Schover et al., 2014). This creates a significant gap in the quality of care for younger women with cancer. In order to provide comprehensive care to them, oncology social workers need to be knowledgeable about the psychosocial needs of younger generations of women. Although increasing attention has been paid to young adult issues, at the moment only one training program for health care professionals exists (Vadaparampil, Hutchins \& Quinn, 2013). Previous research has shown that poor communication with patients and partners is associated with psychological distress and reduced satisfaction with care (Regan, Levesque, Lambert, \& Kelly, 2015b). Hence, 
enhancing health care professionals' preparation in discussing psychosocial issues could provide significant benefits.

Furthermore, the identification of unique needs and stressors requires the utilization of screening and assessment strategies able to capture the life experience of younger women and their well-being, in order to promote effective and timely referrals. Close relationships, emotional functioning, body image, sexuality, and reproductive health should be examined with great attention within this group (Lawson, Klock, Pavone, Hirshfield-Cytron, Smith \& Kazer, 2014; Ruddy \& Partridge, 2012), given the potential long-term consequences for the quality of life of the patient, and the well-being of the couple. As part of the recently implemented mandatory distress screening protocol, some of these areas are only marginally targeted. It is then recommended to integrate instruments developed specifically for these purposes like the Young Women with Breast Cancer Inventory (Christophe et al., 2015b), the Cancer Needs Questionnaire - Young People (Clinton-McHarg, Carey, Sanson-Fisher, D'este \& Shakeshaft, 2012) or the Reproductive Concerns Scale (Wenzel et al., 2005). Aubin and Perez (2015) have recently developed a "clinical toolbox" to assess sexuality in younger patients, which include clinical interviews and self -report questionnaires that can be easily administered.

When compared with older partners, younger caregivers were more negatively affected by the cancer diagnosis. Hence, social workers should be attentive to their experience as well. Screening and assessment should be inclusive of the significant other in order to identify individuals who may have difficulties coping with the patient's diagnosis and its demands. Among the numerous questionnaires available to assess caregivers' quality of life and distress (Edwards \& Ung, 2002), Vanlemmens et al. (2012) 
have advocated for the creation of instruments that focus on the experience of younger partners and recently introduced the Partner-YW-BC Inventory. Since the questionnaire has been developed in France, an adaptation and validation is necessary because of crosscultural differences. However, areas of quality of life investigated by the instrument can be used to develop questions to guide the conversation with younger partners.

Since younger patients and partners' mutuality contributes to dyadic coping, psychosocial interventions should address the dyad as a unit of intervention. Over the last two decades, several couple-based interventions have been developed and tested in RCTs (Badr \& Krebs, 2012; Baik \& Adams; 2011; Regan et al., 2012; Scott \& Kayser, 2008; Zimmermann, Heinrichs, \& Baucom, 2007). Despite more investigation is warranted to evaluate their effectiveness and application in practice settings, authors have found greater improvements in dyadic coping for younger couples (Kayser \& Acquati, 2011). Programs aimed at supporting younger dyads coping with breast cancer should assist participants identifying their relationships qualities, positive and negative coping patterns and their impact on their reciprocal quality of life. Through this experience younger couples should be taught how to modify dysfunctional coping patterns and to establish new coping repertoires, while enhancing couple resilience within the safe setting of a therapeutic intervention (Skerrett \& Fergus, 2015). It is, however, necessary to adapt existing protocols to target topics that are relevant for younger couples in the early years of marriage or cohabitating couples who are preparing for marriage (Ponzetti, 2016). Additional issues could include: social relations with families of origin and the extensive supportive network, financial planning, fertility preservation options and transition to parenthood for couples interested in having children after cancer or strategies to cope 
with cancer-related infertility. While preliminary data indicate beneficial changes in communication, cancer-related discussion, greater closeness and higher relationship strengths for younger dyads (Fergus et al., 2014; Fergus et al., 2015), it will be important to further investigate factors associated with positive results, timing and the mechanism for therapeutic change (Revenson \& DeLongis, 2011; Revenson \& Lepore, 2012).

As improvements in early detection and treatment have increased the survival rate, cancer is becoming a chronic condition. Younger breast cancer survivors experience late treatment-related side effects -often including physical, psychosocial, and cognitive issues- and fear of recurrence or of new malignancies (Damaskos \& Parry, 2015; Howlader et al., 2011; Siegel, Naishadham \& Jemal, 2013; Valdivieso, Kujawa, Jones \& Baker, 2012). Very debilitating long-term effects of chemotherapy include cardiac toxicity, secondary leukemia, affected cognitive function and neurotoxicity (Azim, Azambuja, Colozza, Bines \& Piccart, 2011). Hence, younger women should be offered care along the cancer continuum through individual, couples and group approaches. Indications from the literature suggest that cognitive behavioral approaches had the highest success in promoting better coping skills and this can represent a starting point for future experiences (Damaskos \& Parry, 2015; Traeger, Greer, Fernandez-Robles, Temeland \& Pirl, 2010). Furthermore, psychosocial issues should be incorporated in survivorship care plans to promote better coordination of care (ACOS COC, 2012).

\section{Limitations}

The study presents several limitations. First, the small sample size affects the generalization of results to other groups. A more appropriately powered sample is needed 
to investigate the differential impact of breast cancer among patients and partners across the developmental trajectory. Although difficulties in the recruitment of couples for research are well-established by the literature (Fredman et al., 2009; Hagedoorn et al., 2015; Kenny et al., 2006; Regan, Lambert, \& Kelly, 2013), the limited sample and the use of different instruments to measure quality of life of patients and partners negatively impacted the data analysis strategy and the possibility to test more complex models of dyadic data analysis (Ledermann \& Kenny, 2012; Ledermann \& Macho, 2014;

Ledermann, Macho \& Kenny, 2011). For example, in the original conceptual framework it was planned to control for socio-demographic variables. Similarly, dyadic data analysis can also be conducted using Structural Equation Modeling. However, most authors recommend this statistical methodology with sample size of at least 200 subjects (Kline, 2011; Raykov \& Marcoulides, 2006). Furthermore, in the current study the identification of "younger" and "older" dyads was derived only by the age of the patient at diagnosis. Future studies can benefit from a more in-depth exploration of the role of developmental stages, duration of relationship and cohort effects, and how these interact with couples' coping with cancer (Baucom et al., 2012; Revenson \& Lepore, 2012).

In addition, the sample was largely homogeneous. The absence of diversity in regards to race, socio-economic status, sexual orientation and level of education represents a limitation of the study. Although the two institutions where the research was conducted were selected because they were serving a metropolitan area characterized by diversity and the research materials were available in multiple languages, the sample mirrors the type of patients seeking care at these institutions. Other strategies to increase 
diversity would have been to include online study materials (especially to target younger participants) and to partner with a community-based or advocacy organization.

This project used a cross sectional design to assess individuals' and couples' adjustment to the patients' illness in the first three months from diagnosis. Since coping with cancer is a process that changes and evolves with time and along the cancer care continuum, individuals' and couples' adaptation to illness is not static. Hence, it is not possible to elaborate whether the adjustment of younger patients and partners is consistently more affected over time than the one of older participants.

While dyadic coping has been consistently associated to stress reduction and improved quality of the relation (Falconier et al., 2015), marital satisfaction was not included in the original research protocol. Thus, it was not possible to examine the relationship between dyadic coping and marital satisfaction in the two groups, to test whether this association is maintained also in younger couples, or if worse outcomes are reported for those who are already experiencing difficulties in their relationships. Similarly, while some items in the instruments address sexuality, it would have been interesting to include scales about body image and sexual functioning, next to anxiety and depression inventories.

In the present study dyadic coping was measured using the Dyadic Coping Scale. While examining the study variables of interest, very high correlations were identified between common and positive dyadic coping ( $r=.90$ for patients, $r=.93$ for partners), which raised some concerns for the use of this two subscales in the analyses. Similarly, an element that suggests the need for further reflection on the measurement of mutuality and dyadic coping is the presence of very large actor effects in the APIM analysis. 
However, the scale has been recently reviewed and re-named Dyadic Coping Inventory (DCI, Bodenmann, 2008). It includes now 37 items and it is organized around four subscales (delegated, supportive, stress communication, and negative dyadic coping) which can be grouped into two total scores (positive and negative dyadic coping). While the DCI has been extensively used in European countries (Ledermann et al, 2010; Falconier, Nussbeck, \& Bodenmann, 2013; Vedes, Nussbeck, Bodenmann, Lind, \& Ferreira, 2013), the first validation study for the US population was published while this research project was completed (Levesque, Lafontaine, Caron \& Fitzpatrick, 2014). The inclusion of this new instrument in future research projects is recommended, also to test if current results are replicated. In particular, the distinction between dyadic coping by oneself and by the partner may contribute to clarify the results reported by younger dyads.

Finally, since breast cancer in younger women is associated with more aggressive biological characteristics, advanced stage of disease, and worse prognosis (Ademuyiwa et al., 2015; American Cancer Society, 2014), it would have been interesting to examine differences for staging and treatment type, and to test whether these differences may have influenced the quality of life of patients as well as their partners'.

\section{Future Directions}

While most previous studies on younger women diagnosed with breast cancer failed to include the partner, and the attention to the developmental trajectory of dyadic coping over the life-span is a significant gap in the current literature about couples coping with cancer, the present investigation has provided important indications about the unique 
experience of younger patients and their partners. It also expands our understanding of the inter-relationship between mutuality and dyadic coping, and how they both play important roles in optimal adjustment to a cancer diagnosis. Despite the limitations listed earlier, these results represent the starting point of a future program of research in psychosocial oncology aimed at improving the quality of life of younger women and their partners, and the provision of quality cancer care.

The lower quality of life and emotional well-being of both younger patients and partners (as compared to older patients and partners) suggests that additional studies, both cross-sectional and longitudinal, are needed to examine the differential impact cancer has on couples across the developmental trajectory and to provide confirmation to these findings. Building on larger samples and on dyadic study conceptualizations, this research will lead to better understand sources of stress and relational impairment experienced by younger dyads, which will be used to inform future interventions. Furthermore, future studies will contribute to the identification of individual (i.e.: personality characteristics) and relational characteristics (i.e.: closeness, strength of the relationship; authenticity) associated to better adjustment during a stage of life currently overlooked by the literature (Saita, Acquati, \& Kayser, 2015). This will allow for the early identification of individuals and couples who are more vulnerable to a negative adaption to the illness. Similarly, it will be critical to target different types of cancer and particularly cancers that affect both genders, with the long-term goal to examine the effect of gender, role and dyad characteristics over younger dyads' emotional well-being and adaptation to cancer (Hagedoorn et al., 2008). 
One of the most interesting findings from the study is the absence of association between dyadic coping and emotional well-being of younger partners, which has raised numerous questions about factors that contribute to the quality of life of younger caregivers. Given the few precedents in the literature, qualitative investigations will be essential to elaborate more on the meanings associated to the cancer diagnosis of the patient, the new role of caregiver, and to identify significant stressors and sources of support for this group (for example, the role of the larger supportive network/work environment). Findings from these studies will contribute to the development of instruments and supportive interventions.

Recently, the adverse impact of out-of-pocket health costs has been labeled as the financial toxicity of cancer (Zafar et al., 2013). This financial burden negatively affects quality of life of patients and survivors, in the form of worse physical and mental wellbeing, poor adherence and utilization of care (Azzani et al., 2015; Chino et al., 2014; Fenn et al., 2014; Sharp, Carsin \& Timmons, 2013). Within this literature, alarming are the findings about the younger group. Not only younger patients have a 10 times higher likelihood of bankruptcy, but cancer survivors in their 30s report significantly worse financial status than those of younger age (Banegas et al., 2016; Landwehr et al., 2016; Ramsey et al., 2013). Future studies are needed to examine the impact of cancer's costs on the well-being of younger patients during the cancer continuum, and also to examine how financial stress affects the relationship of younger patients' and partners. While the literature has recently demonstrated that economic hardship is associated with higher conflict, lower life satisfaction and negative communication in young couples (Halliday 
Hardie \& Lucas, 2010; Haid \& Seiffge-Krenke, 2013; Williamson et al., 2013), no investigation has been conducted about younger couples coping with cancer.

To date, a few studies have explored differences among younger breast cancer patients based on racial and ethnic backgrounds. However, Morrow and colleagues (2014) identified racial disparities in terms of higher financial distress for Black women, while Hispanic women had higher family distress and higher cancer-specific symptoms, compared to White respondents. Additionally, members from minority groups report a critical need for culturally appropriate information and communication strategies from health-care professionals (Colon, 2015; Rivers et al., 2013; Surbone, Zwitter, Rajer, \& Stiefel, 2013). It will be therefore important to investigate differences in the experience of individuals and couples from different racial and socio-economic status, immigrants, with no or inadequate health-care coverage and from segregated neighbors; all factors that contribute to health disparities in cancer care (Elk \& Landrine, 2011; Israel, Eng, Schulz, \& Parker, 2012; Koh, 2009). Methodologically, this can be achieved implementing community-based participatory research (Gehlert \& Coleman, 2010; Israel, Eng, Schulz, \& Parker, 2012). Furthermore, only recently studies started to address the role of culture on dyadic coping (e.g.: Falconier et al., 2013; Kayser et al., 2014).While it is well established that culture significantly shapes the individual reaction and adaptation to illness, the paucity of works on couples coping with cancer indicates a current lack of attention to cultural aspects of coping, and our inability to test whether coping strategies developed in Western cultures are confirmed in collectivistic settings (Fu, $\mathrm{Xu}, \mathrm{Liu}, \&$ Haber, 2008; Tran, 2009). In this direction, the work of Kayser et al. (2014) comparing couples from China, India and the United States identified cultural factors influencing the 
coping process and revealed that some couples are able to transcend those cultural norms to better adjust.

Finally, an interesting area to consider is the role of attachment on younger couples' adaptation to illness and quality of the relationship (Pietromonaco, Uchino, \& DunkelShetter, 2013). Since it is estimated that approximately $55-65 \%$ of adults are secure, $22-$ $30 \%$ avoidant, and 15-20\% ambivalent (Magai, 2008), it is likely that discrepancies exist in the dyad, thus influencing appraisal and coping interactions (Pietromonaco, Feldman Barrett, \& Powers, 2006). Furthermore, parents and their adult children share similarities in dyadic coping responses (Donato et al., 2011; Donato, Iafrate \& Barni, 2013; Iafrate, Donato, \& Bertoni, 2013), with parents acting as role models for the acquisition of dyadic coping. This intergenerational transmission of coping occurs mostly for negative dyadic coping styles and it is registered especially among younger women. These findings have significant implications for younger dyads, as in the early stage of the relationship partners are trying to develop their own identity, norms and roles separated from the dynamics of their families of origin (Carra' Mittini, 1999; Scabini \& Cigoli, 2000; Scabini \& Iafrate, 2003; Scabini \& Rossi, 2012). Future research may explore how the attachment style of the two partners influences their coping with breast cancer and also if more beneficial outcomes are reported by couples where the relationship with the partner has compensated for maladaptive influences of the family of origin.

\section{Conclusion}

Although the psychosocial adaptation to a diagnosis of breast cancer continues to be extensively studied in psychosocial oncology research, the experience of younger women and their partners has received little attention by the literature. Furthermore, a paucity of 
previous works to date have applied a relational perspective to this phenomenon. To our knowledge this was the first study conducted in the United States to focus specifically on how younger patients and partners cope with early stage breast cancer. By comparing their experience to the one of older dyads, results indicate that younger couples are more negatively affected by the cancer diagnosis and its consequences in the first three months from diagnosis. In particular, younger women's social well-being is highly vulnerable to negative forms of dyadic coping. Although actor and partner effects of mutuality indicate that the perception of a supportive relationship contributes to more adaptive and coordinated coping, an aspect of risk is identified in the increased negative dyadic coping reported by younger women when their partners show high display of emotional support.

On a more theoretical level, current findings provided empirical evidence for the Relational-Cultural Model of dyadic coping (Kayser et al., 2007). This has important implications for the conceptualization of dyadic coping, as the available literature has mostly focused on its consequences rather than on the process that originates conjoint forms of coping within the dyad. Since the present study has investigated only one among the relational characteristics identified by the model, further investigations are needed to test the model in its integrity, with the goal to clarify the connection between relational abilities, coping and individual and relational outcomes.

Future studies should continue to provide attention to the developmental trajectory of dyadic coping over the life-span across a variety of cancer types, socio-economic and cultural contexts. Furthermore, younger couples, because of their higher vulnerability to stress coupled with generational and contextual exposure to more numerous stressors (APA, 2015), would benefit from psychosocial interventions able to address their specific 
needs and challenges. These future endeavors should be inspired by the hope and the belief that close relationships can heal and can contribute to greater well-being, despite the uncertainty that accompanies the cancer experience (Cigoli, 2006; Saita, Cigoli, \& Margola, 2009). 


\section{REFERENCES}

Acitelli, L. K., \& Badr, H.J. (2005). My illness or our illness?Attending to the relationship when one partner is ill. In T. A. Revenson, Kayser, K., \& Bodenmann, G. (Ed.), Couples coping with stress: Emerging perspectives on dyadic coping (pp. 121-136). Washington, DC: American Psychological Association.

Acitelli, L.K., Douvan, E., \& Veroff, J. (1993). Perceptions of conflict in the first year of marriage: How important are similarity and understanding? Journal of Social and Personal Relationships, 10(1), 5-19. doi: 10.1177/0265407593101001.

Ademuyiwa, F.O., Gao, F., Hao, L., Morgensztern, D., Aft, R.L., Ma, C., \& Ellis, M.J. (2015). US breast cancer mortality trends in young women according to race. Cancer, 121(9), 1469-1476. doi: 10.1002/cncr.29178.

Ahmad, S., Fergus, K., \& McCarthy, M. (2015) Psychosocial issues experienced by young women with breast cancer: the minority group with the majority of need.Current Opinion in Supportive and Palliative Care, 9(3), 271-278. doi: 10.1097/SPC.0000000000000162.

Alberisio, A., \& Viterbori, P. (2002). Consapevolezza e adattamento. In D. Amadori e. al (Ed.), Psicooncologia. Milano: Masson. 
Aldwin, C. (1991). Does age affect the stress and coping process? Implications of age differences in perceived control. Journal of Gerontology, 46(4), 174-180. doi: 10.1093/geronj/46.4.P174

Aldwin,C.M.(1994). Stress, coping, and development: An Integrative perspective. New York:Guilford

Aldwin, C. (2010). Stress and coping across the Lifespan. In In S. Folkman (Ed.), The Oxford Handbook of Stress, Health and Coping (15-34). New York, NY: Oxford University Press.

Aldwin,C.M., \& Levenson, M.R.(1994). Aging and personality assessment.In M.P.Lawton \& J.Teresi (Eds.), Annual Review of Gerontology/Geriatrics (Vol.14, pp.182-209). New York: Springer.

Aldwin, C.M., Levenson, M.R., \& Spiro, A. (1994).Vulnerability and resilience to combat exposure: Can stress have lifelong effects? Psychology and Aging, 9(1), 33 44. http://dx.doi.org/10.1037/0882-7974.9.1.34.

Aldwin, C.M., Sutton, K., \& Lachman, M. (1996). The development of coping resources in adulthood. Journal of Personality, 64(4), 91-113. doi:10.1111/j.14676494.1996.tb00946.x.

Aldwin, C. M., Skinner, E. A., Taylor, A., \& Zimmer-Gembeck, M. J. (2011). Coping and self-regulation across the lifespan. In K. Fingerman, C. Berg, T. Antonucci, \& J. Smith (Eds.), Handbook of Lifespan Psychology (pp. 561-587). Berlin: Springer.

Aldwin, C.M., Sutton, K., J., Chiara, G., \& Spiro, A. III (1996). Age differences in stress, coping, and appraisal: Findings from the Normative Aging Study. Journal of Gerontology: Psychological Sciences, 51B(4), 179-188. 
Amato, P.R. \&Hohmann-Marriott, B. (2007). A comparison of high- and low-distress marriages that end in divorce. Journal of Marriage and Family, 69, 621-638. doi: 10.1111/j.1741-3737.2007.00396.x.

American Cancer Society (2014). Cancer Treatment \& Survivorship. Facts \& Figures 2014-2015. Atlanta: American Cancer Society.

American Cancer Society (2015). Cancer Facts \& Figures 2015. Atlanta: American Cancer Society.

American Cancer Society (2016). Cancer Facts \& Figures 2016. Atlanta: American Cancer Society.

American College of Surgeons' Commission on Cancer (2012). Cancer program standards 2012: Ensuring patient-centered care (Vol. 2, pp.76-77). Retrieved fromhttps://www.facs.org/ /media/files/quality\%20programs/cancer/coc/programst andards2012.ashx.

American Psychological Association (2015). Stress in America. Paying with our health. Available at https://www.apa.org/news/press/releases/stress/2014/stress-report.pdf.

Anderson, D.J., Yates, P., McCarthy, A., Lang, C.P., Harggraves, M. McCarthy, N. \& Poster-Steel, J. (2011). Younger and older women's concerns and menopause after breast cancer. European Journal of Cancer Care, 20(6), 785-794. doi:

10.1111/j.1365-2354.2011.01282.x

Andrzejczak, E., Markocka-Mączka, K. \& Lewandowski, A. (2013). Partner relationships after mastectomy in women not offered breast reconstruction. Psycho-Oncology, 22(7), 1653-1657. doi: 10.1002/pon.3197 
Anllo, L. M. (2000). Sexual life after breast cancer. Journal of Sex \& Marital Therapy, 26(3), 241-248. doi:10.1080/00926230050084632.

Antoine, C., Vandromme, J., Carly, B., Fastrez, M., Liebens, F., \& Rozenberg, S. (2008). A survey among breast cancer survivors: Treatment of the climacteric after breast cancer. Climacteric, 11(4):322-328. doi: 10.1080/13697130802244422.

Antoine, P., Vanlemmens, L., Fournier, E., Trocme', M., \& Christophe, V. (2012). Young couples' experiences of breast cancer during hormone therapy. A phenomenological interpretative dyadic analysis. Cancer Nursing, 36(3), 213-220. doi: 10.1097/NCC.0b013e31826429a5.

Armer, J., \& Fu, M.R.(2005). Age differences in post-breast cancer lymphedema signs and symptoms. Cancer Nursing, 28(3), 200-207.

Armuand, G.M., Wettergren, L., Rodriguez-Wallberg, K.A., \& Lampic, C.(2014). Desire for children, difficulties achieving a pregnancy, and infertility distress 3 to 7 years after cancer diagnosis. Supportive Care in Cancer, 22(10), 2805-2812. doi: 10.1007/s00520-014-2279-z.

Arndt, V., Merx, H., Sturmer, T., Segmaier, C., Ziegler, H., \& Brenner, H. (2004). Agespecific detriments to quality of life among breast cancer patients one year after diagnosis. European Journal of Cancer, 40(5), 673-680. doi: http://dx.doi.org/10.1016/j.ejca.2003.12.007.

Aubin, S., \& Perez, S.(2015). The Clinician's Toolbox: Assessing the sexual impacts of cancer on Adolescents and Young Adults with Cancer (AYAC). Sexual Medicine, 3(3), 198-212. doi: 10.1002/sm2.75. 
Austin, J., \& Falconier, M. (2013). Spirituality and common dyadic coping: Protective factors from psychological aggression in Latino immigrant couples. Journal of Family Issues, 34(3), 323-346. doi:10.1177/0192513X12452252.

Avis, N.E., Crawford, S., \& Manuel, J. (2004). Psychosocial problems among younger women with breast cancer. Psycho-Oncology, 13(5), 295-308.

Avis, N.E., Crawford, S., \& Manuel, J. (2005). Quality of life among younger women with breast cancer. Journal of Clinical Oncology, 23(15), 3322-3330. doi: 10.1200/JCO.2005.05.130.

Azim, H.A. Jr, de Azambuja, E., Colozza, M., Bines, J., \& Piccart, M.J. (2011). Longterm toxic effects of adjuvant chemotherapy in breast cancer. Annals of Oncology, 22(9), 1939-1947. doi: 10.1093/annonc/mdq683.

Azzani, M., Roslani, A.C., \& Su, T.T. (2015). The perceived cancer-related financial hardship among patients and their families: A systematic review. Supportive Care in Cancer, 23(3), 889-896. doi: 10.1007/s00520-014-2474-y.

Baas, S., \& Schmitt, M. (2004). Die Bedeutung der Einkom-mensverteilung für die Ehezufriedenheit langja "hrig verheir-ateter Ehepaare [The significance of income distribution for marital satisfaction in long-term marriages].Zeitschrift fur Familienforschung, 3, 268-288

Badr, H. (2004). Coping with marital dyads: A contextual perspective on the role of gender and health. Personal Relationship, 11(2), 197-211. doi: 10.1111/j.14756811.2004.00078.x 
Badr, H., \& Krebs, P. (2013). A systematic review and meta-analysis of psychosocial interventions for couples coping with cancer. Psycho-Oncology, 22(8), 1688-1704. doi: 10.1002/pon.3200.

Badr, H., \&.Shen, M.J. (2014). Pain catastrophizing, pain intensity, and dyadic adjustment influence patient and partner depression in metastatic breast cancer. The Clinical Journal of Pain, 30(11), 923-933. doi:10.1097/AJP.0000000000000058.

Badr, H., \& Taylor, L.C. (2008). Effects of relationship maintenance on psychological distress and dyadic adjustment among couples coping with lung cancer. Health Psychology, 27(5), 616-627. doi: 10.1037/0278-6133.27.5.616.

Badr, H., \& Taylor, C. L. (2009). Sexual dysfunction and spousal communication in couples coping with prostate cancer. Psycho-Oncology, 18(7), 735-746. doi: 10.1002/pon.1449.

Badr, H, Carmack, C.L., Kashy, D,A., Cristofanilli, M., \& Revenson, T.A. (2010). Dyadic coping in metastatic breast cancer. Health Psychology, 29(2), 169-180. doi: $10.1037 / \mathrm{a} 0018165$.

Badr, H., Acitelli, L.K., \& Taylor, C.L. (2008). Does talking about their relationship affect couple's marital and psychological adjustment to lung cancer? Journal of Cancer Survivorship, 2(1), 53-64. doi: 10.1007/s11764-008-0044-3.

Baider, L., Ever-Hadani, P., Goldzweig, G., Wygoda, M.R., \& Peretz, T. (2003). Is perceived family support a relevant variable in psychological distress? A sample of prostate and breast cancer couples. Journal of Psychosomatic Research, 55(5), 453 460. doi: http://dx.doi.org/10.1016/S0022-3999(03)00502-6. 
Baik, O. M., \& Adams, K.B. (2011). Improving the well-being of couples facing cancer: A review of couples based psychosocial interventions. Journal of Marital \& Family Therapy, 37(2), 250-266. doi: 10.1111/j.1752-0606.2010.00217.x.

Bakewell, R.T., \& Volker, D.L. (2005). Sexual dysfunction related to the treatment of young women with breast cancer. Clinical Journal of Oncology Nursing.9(6):697702. doi: 10.1188/05.CJON.697-702.

Baltes, P. (1987). Theoretical propositions of life-span developmental psychology: On the dynamics between growth and decline. Developmental Psychology, 23, 611626. http://dx.doi.org/10.1037/0012-1649.23.5.611.

Bandura, A. (1986). Social foundations of though and action: A social cognitive theory. Englewood Cliffs, NJ: Prentice-Hall.

Banegas, M.P., Gery P. Guy, G.P. Jr., de Moor, J.S., Ekwueme, D.U., Virgo, K.S., Kent, E.E., Nutt, S., Zheng, Z., Rechis, R., \& Yabroff, K.R. (2016). For working-age cancer survivors, medical debt and bankruptcy create financial hardships. Health Affairs, 35(1), 54-61. doi: 10.1377/hlthaff.2015.0830.

Bantema-Joppe, E.J., de Bock, G.H., Woltman-van Iersel, M., Busz, D.M., Ranchor, A.V., Langendijk, J.A., Maduro, J.H., \& van den Heuvel., E.R. (2015). The impact of age on changes in quality of life among breast cancer survivors treated with breast-conserving surgery and radiotherapy. British Journal of Cancer, 112(4), 636643. doi: 10.1038/bjc.2014.632.

Banthia, R., Malcarne, V.L., Varni, J.W., Ko, C.M., Sadler, G.R., \& Greenbergs, H.L. (2003). The effects of dyadic strength and coping styles on psychological distress in couples faced with prostate cancer. Journal of Behavioral Medicine, 26(1), 31-52. 
Barbarin, O. A., Hughes, D., \& Chesler, M.A. (1985). Stress, coping, and marital functioning among parents of children with cancer. Journal of Marriage and Family, 47(2), 473-480.

Barnes, H. \& Olson, D.H. (1986). Parent-adolescent communication scale. In D.H. Olson, H.I. McCubbin, H. Barnes, A. Larsen, M. Muxen \& M. Wilson (Eds.). Family Inventories. St. Paul, MN: Family Social Science, University of Minnesota. Barnoy S, Bar-Tal, Y., \& Zisser, B. (2006). Correspondence in informational coping styles: how important is it for cancer patients and their spouses? Personality \& Individual Differences, 41(1), 105-115. doi: 10.1016/j.paid.2005.12.013.

Baron, R.M., \& Kenny, D.A. (1986). The moderator-mediator variable distinction in social psychological research: Conceptual, sterategic, and statistical considerations. Journal of Personality and Social Psychology, 51(6), 1173-1182. http://dx.doi.org/10.1037/0022-3514.51.6.1173.

Baucom, D.H., Kirby, J.S., Pukay-Martin, N.D., Porter, L.S., Fredman, S.J., Gremore, T.M., Keefe, F.J., \& Atkins, D (2012). Men's psychological functioning in the context of women's breast cancer. Journal of Marital and Family Therapy, 38(2), 317-329.doi: 10.1111/j.1752-0606.2009.00133.x.

Baucom, D.H., Porter, L.S., Kirby, J.S., Gremore, T.M., \& Keefe, F.J. (2005). Psychosocial issues confronting young women with breast cancer. Breast Disease, 23(1), 103-113.

Baumeister, R. F., \& Leary, M.R. (1995). The need to belong: Desire for interpersonal attachment as a fundamental human motivation. Psychological Bulletin, 117(3), 497-529. http://dx.doi.org/10.1037/0033-2909.117.3.497. 
Beadle, B.M., Woodward, W.A., \& Buchholz, T.A. (2011). The impact of age on outcome in early-stage breast cancer. Seminars in Radiation Oncology; 21(1), 2634. doi: 10.1016/j.semradonc.2010.09.001.

Beck, L. A., Pietromonaco, P. R., DeVito, C., Powers, S. I., \& Boyle, A. (2014). Congruence between spouses' perceptions and observers' ratings of responsiveness: The role of attachment avoidance. Personality and Social Psychology Bulletin. 40(2), 164-74. doi: 10.1177/0146167213507779.

Beck, L.A., Pietromonaco, P.R., DeBuse, C.J., Powers, S.I., \& Sayer, A.G.(2013). Spouses' attachment pairings predict neuroendocrine, behavioral, and psychological responses to marital conflict. Journal of Personality and Social Psychology. 105(3):388-424. doi: 10.1037/a0033056.

Beck, A.T., Rush, A.J., Shaw, B. F., \& Emery, G (1979). Cognitive Therapy of Depression. New York, NY: The Guilford Press

Beesley, V.L., Price, M.A., Webb, P.M., Australian Ovarian Cancer Study Group, \& Australian Ovarian Cancer Study-Quality of Life Study Investigators (2011). Loss of lifestyle: Health behaviour and weight changes after becoming a caregiver of a family member diagnosed with ovarian cancer. Supportive Care in Cancer, 19(12), 1949-1956. doi: 10.1007/s00520-010-1035-2.

Bekteshi, V., \& Kayser, K. (2013). When a mother has cancer: pathways to relational growth for mothers and daughters coping with cancer. Psycho-Oncology, 22(10), 2379-2385. doi: 10.1002/pon.3299. 
Bell, C.J., Ziner, K.W., \& Chamption, V.L. (2009). Death worries and quality of life in younger braest cancer survivors. Western Journal of Nursing Research, 31(8), 1076-1077. doi: 10.1177/0193945909342241.

Bengston, V. L., \& Allen, K.R., (1993). The life course perspective applied to families over time. In P.Boss, W.Doherty, R. LaRossa, W. Schumm, \& S. Steinmetz (Eds.), Sourcebook of family theories and methods: A contextual approach (pp.469-499). New York, NY: Plenum.

Bengston, V. L., Acock, A.C., Allen, K.R., Dilworth-Anderson, P., \& Klein, D.M. (2005). Sourcebook of Family Theory \& Research. Thousand Oaks: Sage Publications, Inc.

Ben-Zur, H. (2001). Your coping strategy and my distress: inter-spouse perceptions of coping and adjustment among breast cancer patients and their spouses. Families Systems and Health, 19(1), 83-94. doi: 10.1037/h0089465.

Ben-Zur, H., Gilbar, O., \& Lev, S. (2004). Coping with breast cancer: patient, spouse, and dyad models. Psychosomatic Medicine, 63(1), 32-39.

Berg, C. A., \& Upchurch, R. (2007). A developmental-contextual model of couples coping with chronic illness across the adult life span. Psychological Bulletin, 133(6), 920-954. doi:10.1037/0033-2909.133.6.920.

Berg, C. A. W., D.J., Butner, J., Bloor, L., Bradstreet, C., Upchurch, R., \& Patton, G. (2008). Collaborative coping and daily mood in couples dealing with prostate cancer. Psychology and Aging, 23(3), 505-516. doi: 10.1037/a0012687. 
Berg, C. A., Meegan, S. P., \& Deviney, F. P. (1998). A social-contextual model of coping with everyday problems across the life span. International Journal of Behavioral Development, 22(2), 239-261. doi: 10.1080/016502598384360.

Bergelt, C., Koch, U., \& Petersen, C. (2008). Quality of life in partners of patients with cancer. Quality of Life Research, 17(5), 653-663. doi: 10.1007/s11136-008-9349-y.

Berghuis, J. P., \& Stanton, A. L. (2002). Adjustment to a dyadic stressor: A longitudinal study of coping and depressive symptoms in infertile couples over an insemination attempt. Journal of Consulting and Clinical Psychology, 70(2), 433-438. doi: 10.1037//0022-006x.70.2.433.

Bertoni, A., Barni, D., Bodenmann, G, Charvoz, L., Gagliardi, S., Iafrate, R., \& Rosnati, R. (2007). Comunicazione dello stress, coping diadico e benessere della coppia: Uno studio cross sectional e cross nazionale [Stress communication, dyadic coping and couple satisfaction: A cross-sectional and cross-cultural study]. Età Evolutiva, $86,58-66$.

Bifulco, G., De Rosa, N., Tornesello, M.L., Piccoli, R., Bertrando, A., Lavitola. G., Morra, I., Di Spiezio Sardo, A, Buonaguro, F.M., \& Nappi, C. (2012). Quality of life, lifestyle behavior and employment experience: a comparison between young and midlife survivors of gynecology early stage cancers. Gynecologic Oncology, 124(3), 444-451. doi: 10.1016/j.ygyno.2011.11.033.

Biglia, N., Moggio, G., Peano, E., Sgandurra, P., Ponzone, R., Nappi, R.E., \& Sismondi, P. (2010). Effects of surgical and adjuvant therapies for breast cancer on sexuality, cognitive functions, and body weight. Journal of Sexual Medicine, 7(5), 1891-1990. doi: 10.1111/j.1743-6109.2010.01725.x. 
Binik, Y.M., Chowanec, G.D., \& Devins, G.M. (1990). Marital role strain, illness intrusiveness, and their impact on marital and individual adjustment in end-stage renal disease. Psychology \& Health, 4(3), 245-257. doi:10.1080/08870449008400394.

Birditt, K.S., \& Fingerman, K.L. (2003). Age and gender differences in adults' descriptions of emotional reactions to interpersonal problems. The Journals of Gerontology Series B: Psychological Sciences and Social Sciences, 58(4), 237-245. doi: 10.1093/geronb/58.4.P237.

Birditt, K.S., Brown, E., Orbuch, T.L., \& McIlvane, J.M. (2010). Marital conflict behaviors and implications for divorce over 16 years. Journal of Marriage and Family, 72(5), 1188-1204. doi: 10.1111/j.1741-3737.2010.00758.x

Blanchard-Fields, F., Sulsky, L.,\& Robinson-Whelen, S. (1991). Moderating effects of age and context on the relationship between gender, sex role differences, and coping. Sex Roles, 25(11), 645-660.

Blanchard-Fields, F., \& Coats, A. H. (2008). The experience of anger and sadness in everyday problems impacts age differences in emotion regulation.Developmental Psychology, 44(6), 1547-1556. http://dx.doi.org/10.1037/a0013915.

Bloom, J.R., D’Onofrio, C., Banks, P., et al. (1999). A Psycho-Educational group intervention for young women with breast cancer: Design and process evaluation. Cancer, Research \& Therapy Control, 8 (1-2), 93-102.

Bloom, J.R., Stewart, S.L., Chang, S., \& Banks, P.J. (2004). Then and now: quality of life of young breast cancer survivors. Psycho-Oncology, 13(3), 147-160. 
Bloom, J.R., Stewart, S.L., D’Onofrio, C.N., Luce, J., \& Banks, P.J. (2008). Addressing the needs of young breast cancer survivors at the 5 year milestone: Can a shortterm, low intensity intervention produce change? Journal of Cancer Survivorship. 2(3), 190-204. doi: 10.1007/s11764-008-0058-x

Bloom, J.R., Stewart, S.L., Johnston, M., \& Banks, P. (1998). Intrusiveness of illness and quality of life in young women with breast cancer. Psycho-Oncology, 7(2), 89-100.

Bloom, J.R., Stewart, S.L., Johnston, M., Banks, P., \& Fobair, P. (2001). Sources of support and the physical and mental well-being of young women with breast cancer. Social Science \& Medicine, 53(11), 1513-1524. doi:10.1016/S0277-9536(00)004408.

Bloom, J.R., Stewart, SL, Oakley-Girvan, I., Banks, P.J., \& Shema, S. (2012). Quality of life of younger breast cancer survivors: Persistence of problems and sense of wellbeing. Psycho-Oncology, 21(6), 655-665. doi: 10.1002/pon.1965.

Bodenmann, G. (1995a). A systemic-transactional view of stress and coping in couples. Swiss Journal of Psychology, 54, 34-49.

Bodenmann, G. (1995b). Dyadische Coping und Partnerschaftszufriedenheit [Dyadic coping and marital satisfaction]. PsychologischeBeiträge, 37, 72-89.

Bodenmann, G. (1997). Dyadic coping: A systemic-transactional view of stress and coping among couples: Theory and empirical findings. European Review of Applied Psychology, 47, 137-140.

Bodenmann, G. (2000). Stress und Coping bei Paaren [Stress and coping in couples]. Göttingen, Germany: Hogrefe. 
Bodenmann, G. (2005). Dyadic coping and its significance for marital functioning. In T. A. Revenson, Kayser, K., \& Bodenmann, G. (Ed.), Couples coping with stress: Emerging perspectives on dyadic coping (pp. 35-50). Washington, DC: American Psychological Association.

Bodenmann, G. (2007). Dyadisches Coping Inventar. Testmanual [Dyadic Coping Inventory. Test manual].Bern, Switzerland: Huber.

Bodenmann, G. (2008). Dyadisches Coping Inventar (DCI) [Dyadic Coping Inventory]. Bern, Switzerland: Huber; German.

Bodenmann, G. (2008). Improving dyadic coping in marital distress prevention programs and marital therapy. In L. VandeCreek, \& J. B. Allen (Eds). Innovations in clinical practice: Focus on group, couples, \& family therapy. Innovations in clinical practice (pp. 235-251). Sarasota, FL: Professional Resource Press/Professional Resource Exchange.

Bodenmann, G., Atkins, D.C., Schär, M., \& Poffet, V. (2010). The association between daily stress and sexual activity. Journal of Family Psychology, 24(3), 271-279. doi: 10.1037/a0019365.

Bodenmann, G., \& Bertoni, A. (2004). Promuovere le competenze della coppia. II Couple Coping Enhancement Training [Promoting couple's competencies. The Couple Coping Enhancement Training]. Roma: Carocci.

Bodenmann, G., Bradbury, T.N., \& Pihet, S. (2009). Relative contributions of treatmentrelated changes in communication skills and dyadic coping skills to the longitudinal course of marriage in the framework of marital distress prevention. Journal of Divorce \& Remarriage, 50(1), 1-21. doi: 10.1080/10502550802365391. 
Bodenmann, G., Charvoz, L., Widmer, K., \& Bradbury, T.N. (2004). Differences in individual and dyadic coping among low and high depressed, partially remitted, and nondepressed persons. Journal of Psychopathology and Behavioral Assessment, 26(2), 75-85.

Bodenmann, G., \& Cina., A. (2006). Stress and coping among stable-satisfied, stabledistressed and separated/divorced swiss couples. Journal of Divorce \& Remarriage, 44(1), 71-89. doi: 10.1300/J087v44n01_04.

Bodenmann, G., Cina, A., \& Schwerzmann, S. (2001). Individuelle und dyadische copingressourcen bei depressiven [Individual and dyadic coping resources in depressives]. Zeilschrift für Klinische Psychologie und Psychotherapie, 30, 194203.

Bodenmann, G., Hilpert, P., Nussbeck, F. W., Bradbury, T. N. (2014). Enhancement of couples' communication and dyadic coping by a self-directed approach: A randomized controlled trial. Journal of Consulting and Clinical Psychology, 82(4), 580-591. http://dx.doi.org/10.1037/a0036356

Bodenmann, G., \& Langenick, D. (1996). The influence of stress and coping on marital quality and stability:A three-year longitudinal study. Fribourg, Switzerland: University of Fribourg.

Bodenmann, G., Meuwly, N., Bradbury, T. N., Gmelch, S., \& Ledermann, T. (2010). Stress, anger, and verbal aggression in intimate relationships: Moderating effects of individual and dyadic coping. Journal of Social and Personal Relationships, 27, 408-424. doi: 10.1177/0265407510361616 
Bodenmann, G., Meuwly, N.,Germann, J., Nussbeck, F., Heinrichs, M., \& Bradbury, T. (2015). Effects of stress on the social support provided by mean and women in intimate relationships. Psychological Science, September 4, 2015 doi:0956797615594616.

Bodenmann, G., Meuwly, N., \& Kayser, K. (2011). Two conceptualizations of dyadic coping and their potential for predicting relationship quality and indi individual wellbeing. European Psychologist, 16, 255-266. doi:10.1027/1016-9040/a000068.

Bodenmann, G., Perrez, M., Charvoz, L., Cina, A., \& Widmer, K. (2002). The effectiveness of coping focused prevention approach: A two-year longitudinal study. Swiss Journal of Psychology, 61, 195-202. doi: 10.1024/1421-0185.61.4.195.

Bodenmann, G., Pihet, S., \& Kayser, K. (2006). The relationship between dyadic coping and marital quality: A 2-year longitudinal study. Journal of Family Psychology, 20(3), 485-493. http://dx.doi.org/10.1037/0893-3200.20.3.485.

Bodenmann, G., Pihet, S., Shantinath, S., Cina, A., \& Widmer, K. (2006). Improving dyadic coping in couples with a stress-oriented approach: A two-year longitudinal study. Behavior Modification, 30(5), 571-597. doi: 10.1177/0145445504269902.

Bodenmann, G., Plancherel, B., Beach, S.R.H., et al. (2008). Effects of coping oriented couples therapy on depression: a randomized clinical trial. Journal of Consulting \& Clinical Psychology, 76, 499-454.

Bodenmann,G., \& Randall, A.K.(2012). Common factors in the enhancement of dyadic coping. Behavior Therapy,43,88-98.doi:10.1016/j.beth.2011.04.003. 
Bodenmann, G., \& Shantinath, S. D. (2004). The Couples Coping Enhancement Training (CCET): A new approach to prevention of marital distress based upon stress and coping. Family Process, 53, 477-484.

Bodenmann, G. \& Widmer, K. (2000). Stressbewältigung im Alter: Ein Vergleich von Paaren jüngeren, mittleren und höheren Alters. Zeitschrift für Gerontologie und Geriatrie, 33,217-228.

Bodenmann, G., Widmer, K., Charvoz, L., \& Bradbury, T. N. (2004). Differences in individual and dyadic coping in depressed, non-depressed and remitted persons. Journal of Psychopathology and Behavioral Assessment, 26(2), 75-85. doi:10.1023/B:JOBA.0000013655.45146.47.

Bolger, N., DeLongis, A., Kessler, R.C., \& Schilling, E.A. (1989). Effects of daily stress on negative mood. Journal of Personality and Social Psychology, 57(5), 808-818. doi: 10.1037/0022-3514.57.5.808.

Bond, M., Gardiner, S.T., Christian, J., \& Sigel, J.J. (1983). An empirical examination of defense mechanisms. Archives of General Psychiatry, 40, 33-38.

Borg, C. \& Hallberg, I.R.(2006). Life satisfaction among informal caregivers in comparison with non-caregivers Scandinavian Journal of Caring Sciences, 20(4), 427-438. doi: 10.1111/j.1471-6712.2006.00424.x.

Borstelmann, N.A., Rosenberg, S.M., Ruddy, K.J., Tamimi, R.M., Gelber, S., Schapira, L., Come, S., Borges, V., Morgan, E., \& Partridge A.H. (2015). Partner support and anxiety in young women with breast cancer. Psycho-Oncology, 24(12), 1679-1685. doi: 10.1002/pon.3780.

Bowen, M. (1978). Family therapy in clinical practice. New York, NY: Aronson. 
Bowlby, J. (1969). Attachment and loss: Vol. 1. Attachment. New York, NY: Basic Books.

Bowlby, J. (1988). A secure base: Parent-child attachment and healthy human development. New York, NY: Basic Books.

Bradbury, T.N., \& Karney, B.R. (2014). Intimate Relationships. New York, NY: W.W. Norton \& Company.

Brady, M.J., Cella, D.F., Mo, F., Bonomi, A.E., Tulsky, D.S., Lloyd, S.R., Deasy, S., Cobleigh, M., \& Shiomoto, G. (1997). Reliability and validity of the Functional Assessment of Cancer Therapy-Breast quality-of-life instrument. Journal of Clinical Oncology, 15(3), 974-86.

Bramlett, M.D., \& Mosher,W.D. (2002). Cohabitation, marriage, divorce, and remarriage in the United States. (Vital and Health Statistics, series 23, No.22) Washington, DC: U.S. Government Printing Office.

Braun, M., Hales, S., Gilad, L., Mikulicer, M., Rydall, A., \& Rodin, G. (2012). Caregiving styles and attachment orientations in couples facing advanced cancer. Psycho-Oncology, 21(9), 935-943. doi: 10.1002/pon.1988.

Braun, M., Mikulincer, M., Gagliese, L., Moore, M., \& Rodin, G. (2004). Depression and demoralization of spouse caregivers of cancer patients. Paper presented at the 14th Annual Provincial Conference on Palliative and End-of-Life Care, Toronto, Canada.

Braun, M., Mikulincer, M., Rydall, A., Walsh, A., \& Rodin, G. (2007). Hidden morbidity in cancer: Spouse caregivers. Journal of Clinical Oncology, 25(30), 4829-4834. doi: 10.1200/JCO.2006.10.0909. 
Breitenstein, C., Milek, A., Nussbeck, F., \& Bodenmann (2015). Stress, Dyadic Coping, and Relationship Satisfaction in Late Adolescent Couples. Poster presented at the International Association of Relationship Research, At Amsterdam, NLD

Brennan, M., Gormally, J., Butow, P., Boyle, F., \& Spillane, A. (2014). Survivorship care plans in cancer: A systematic review of care plan outcomes. British Journal of Cancer, 111(10),1899-1908. doi:10.1038/bjc.2014.505.

Brucker, P.S., Yost, K., Cashy, J., Webster, K., \& Cella, D. (2005). General population and cancer patient norms for the Functional Assessment of Cancer Therapy-General (FACT-G). Evaluation \& the Health Professions, 28(2), 192-211. doi: 10.1177/0163278705275341.

Buchanan, N., Roland, K.B., Rodriguez, J.L., Miller, J.W., \& Fairley, T. (2013). Opportunities for Public Health communication, intervention, and future research on breast cancer in younger women. Journal of Women's Health, 22(4), 293-298. doi: 10.1089/jwh.2012.4239.

Burman, B., \& Margolin, G. (1992). Analysis of the association between marital relationship and health problems: An interactional perspective. Psychological Bulletin, 112(1), 39-63. http://dx.doi.org/10.1037/0033-2909.112.1.39.

Burwell, S.R., Case, L.D., Kaelin, C., \& Avis, N.E. (2006). Sexual problems in younger women after breast cancer surgery. Journal of Clinical Oncology, 24(18), 28152821. doi: 10.1200/JCO.2005.04.2499.

Burwell, S. R., Templeton, B., Kennedy, K.S., \& Zak-Hunter, L. (2008). Feministinformed medical family therapy principles and techniques across biopsychosocial 
system levels for younger women with breast cancer and their partners. Journal of Feminist Family Therapy, 20(2), 99-125. doi:10.1080/08952830802023276.

Cannon, W. B. (1932). The wisdom of the body. New York. NY: W. W. Norton.

Carr, S. V. (2013). The impact of cancer and its therapies on body image and sexuality. In B. I. Carr, \& Steel, J. (Ed.), Psychological Aspects of Cancer. A Guide to Emotional and Psychological Consequences of Cancer, their Causes and their Management (pp. 199-212). New York, NY: Springer.

Carr, D., Freedman, V. A., Cornman, J. C., \& Schwarz, N. (2014). Happy marriage, happy life? Marital quality and subjective well-being in later life. Journal of Marriage and Family, 76, 930-948. doi:10.1111/jomf.12133.

Carr, B. I., \& Steel, J. (2013). Psychological Aspects of Cancer. A Guide to Emotional and Psychological Consequences of Cancer, Their Causes and Their Management. New York, NY: Springer.

Carra' Mittini, E. (1999). Una famiglia, tre famiglie. La famiglia giovane nella trama delle generazioni. Milano: Unicopli.

Carstensen, L. L., Edelstein, B. A., \& Dornbrand, L. (1996) Practical Handbook of Clinical Gerontology. Sage Publications.

Carstensen, L. L., Fung, H., \& Charles, S. (2003) Socioemotional selectivity theory and the regulation of emotion in the second half of life. Motivation and Emotion, 27(2), 103-123. doi:10.1023/A:1024569803230.

Carstensen, L. L., Gottman, J. M., \& Levenson, R. W. (1995). Emotional behavior in long-term marriage. Psychology and Aging, 10(1), 140-149. 
Carstensen, L.L., Graff, J., Levenson, R.W., \& Gottman, J.M. (1996). Affect in intimate relationships: The developmental course of marriage. In: Magai C, McFadden SH, editors. Handbook of emotion, adult development, and aging (pp. 227-247). San Diego, CA: Academic Press.

Carstensen, L.L., Turan, B., Scheibe, S., Ram, N., Ersner-Hershfield. H., SamanezLarkin, G.R., Brooks, K.P., \& Nesselroade, J.R. (2011). Emotional experience improves with age: evidence based on over 10 years of experience sampling. Psychology \& Aging, 26(1), 21-33. doi: 10.1037/a0021285.

Carter R., \& McGoldrick, C. (1999). Communication in couples with breast cancer. doi: http://healthlink.mcw.edu/article.

Carver, C. S., \& Connor-Smith, J. (2010). Personality and coping. Annual Review of Psychology, 61, 679-704. doi:10.1146/annurev.psych.093008.100352.

Carver, C. S., \& Scheier, M. F. (1982). Control Theory: A useful conceptual framework for personality-social, clinical, and health psychology. Psychological Bulletin, 92(1), 111-135. doi:10.1037/0033-2909.92.1.111.

Carver, C.S., Smith, R.G., Petronis, V.M., \& Antoni, M.H. (2006). Quality of life among long-term survivors of breast cancer: Different types of antecedents predict different classes of outcomes. Psycho-Oncology; 15(9),749-758. doi:10.1002/pon.1006.

Cassidy, T., Giles, M., \& McLaughlin, M. (2014). Benefit finding and resilience in child caregivers. British Journal of Health Psychology, 19(3), 606-618. doi: 10.1111/bjhp.12059. 
Cataldo, J.K., Paul, S., Cooper, B., Skerman, H., Alexander, K., Aouizerat, B., Blackman, V., Merriman, J., Dunn, L., Ritchie, C., Yates, P., \& Miaskowski, C. (2013). Differences in the symptom experience of older versus younger oncology outpatients: A cross-sectional study. BMC Cancer, 13(6). doi:10.1186/1471-240713-6. http://www.biomedcentral.com/1471-2407/13/6.

Caughlin, J. P. (2002). The demand/withdraw pattern of communication as a predictor of marital satisfaction over time. Unresolved issuesand future directions. Human Communication Research, 28(1), 49-85. doi: 10.1111/j.1468-2958.2002.tb00798.x.

Cebeci, F., Yangin, H.B., \& Tekeli, A. (2010). Determination of changes in the sexual lives of young women receiving breast cancer treatment: A qualitative study. Sexuality \& Disability, 28(4), 255-264. doi: 10.1007/s11195-010-9169-1.

Cella, D.F. (1994). Quality of life concepts and definition. Journal of Pain \& Symptom Management, 9(3), 186-192.

Cella, D.F. (1995). Methods and problems in measuring quality of life. Supportive Care in Cancer, 3(1), 11-22.

Cella, D.F., Tulsky, D.S., Gray, G., Sarafian, B., Lloyd, S., Linn, E., Bonomi, A., Silberman, M., Yellen, S.B., Winicour, P., Brannon, J., Eckberg, K., Purl, S., Blendowski, C., Goodman, M., Barnicle, M., Stewart, I., McHale, M., Bonomi, P., Kaplan, E., Taylor, S., Thomas, C., \& Harris, J. (1993). The Functional Assessment of Cancer Therapy (FACT) scale: Development and validation of the general measure. Journal of Clinical Oncology, 11(3), 570-579.

Chalmers, L., \& Milan, A. (2005) Marital satisfaction during the retirement years. Canadian Social Trends, 11, 14-17. Available at http://www- 
psh.nearnorthschools.ca/Technology/BadgerS/Articlesforreadingassignments/Marit alsatisfactionduringtheretirementyears.pdf.

Chambers, S.K., Lowe, A., Hyde, M.K., Zajdlewicz, L., Gardiner, R.A., Sandoe, D., \& Dunn, J. (2014). Defining young in the context of prostate cancer. American Journal of Men's Health, 9(2), 103-114. doi: 10.1177/1557988314529991

Champion, V.L., Wagner, L.I., Monahan, P.O., Daggy, J., Smith, L., Cohee, A., Ziner, K.W., Haase, J.E., Miller, K.D., Pradhan, K., Unverzagt, F.W., Cella, D., Ansari, B., \& Sledge, G.W (2014). Comparison of younger and older breast cancer survivors and age-matched controls on specific and overall quality of life. Cancer, 120(15), 2237- 2246. doi: 10.1002/cncr.2873.

Charif, A.B., Bouhnik, A.D., Rey, D., Provansal, M., Courbiere, B., Spire, B., \& Mancini, J. (2015). Satisfaction with fertility- and sexuality-related information in young women with breast cancer-ELIPPSE40 cohort. BMC Cancer, 15, 572-583. doi:10.1186/s12885-015-1542-0

Charles, S.T., \& Piazza, J.R. (2007). Memories of social interactions: Age differences in emotional intensity. Psychology and Aging, 22(2), 300-309. http://dx.doi.org/10.1037/0882-7974.22.2.300.

Chino, F., Peppercorn, J., Taylor, D., Lu, Y., Samsa, G., Abernethy, A. P., \& Zafar, S. Y. (2014). Self-reported financial burden and satisfaction with care among patients with cancer. The Oncologist, 19(1), 414-420. doi: 10.1634/theoncologist.20130374.

Christophe, V., Duprez, C., Congard, A., Antoine, P., Lesur, A., Fournier, E \& Vanlemmens, L. (2015a). The subjective experience of young women with non- 
metastatic breast cancer: the Young Women with Breast Cancer Inventory. Health and Quality of Life Outcomes, 13:73. doi 10.1186/s12955-015-0273-x.

Christophe, V., Duprez, C., Congard, A., Fournier, E., Lesur, A., Antoine, P., \& Vanlemmens, L. (2015b). Evaluate the subjective experience of the disease and its treatment in partners of young women with non-metastatic breast cancer. European Journal of Cancer Care. doi: 10.1111/ecc.12327.

Chung, C., \& Hwang, E. (2012). Couples' experiences of breast cancer in Korea: a descriptive qualitative study. Cancer Nursing, 35(3), 211-220. doi: 10.1097/NCC.0b013e31822a60db.

Chung, M.L., Moser, D.K., Lennie, T.A., \& Rayens, M.K. (2009). The effects of depressive symptoms and anxiety on quality of life in patients with heart failure and their spouses: Testing dyadic dynamics using Actor-Partner Interdependence Model. Journal of Psychosomatic Research, 67(1), 29035. doi:

10.1016/j.jpsychores.2009.01.009

Cigoli, V. (2006). L'albero della discendenza. Clinica dei corpi familiari. Milano: FrancoAngeli.

Cimprich, B., Ronis, D.L., \& Marinez-Ramos, G. (2002). Age at diagnosis and quality of life in breast cancer survivors. Cancer Practice, 10(2), 85-93. doi:10.1046/j.15235394.2002.102006.x.

Cleek, M. G., \& Pearson, T.A. (1985). Perceived causes of divorce: An analysis of interrelationships. Journal of Marriage \& the Family, 47(1), 179-183. doi: $10.2307 / 352080$. 
Clinton-McHarg, T., Carey, M., Sanson-Fisher, R., D'Este, C., \& Shakeshaft, A. (2012). Preliminary development and psychometric evaluation of an unmet needs measure for adolescents and young adults with cancer: the Cancer Needs Questionnaire Young People (CNQ-YP). Health and Quality of Life Outcomes, 10:13. doi: 10.1186/1477-7525-10-13.

Coan, J.A., Kasle, S., Jackson, A., Schaefer, H.S., \& Davidson, R.J. (2013). Mutuality and the social regulation of neural threat responding. Attachment \& Human Development, 15(3), 303-315. http://dx.doi.org/10.1080/14616734.2013.782656.

Cochrane, B.B., Lewis, F.M., \& Griffith, K.A. (2011). Exploring a diffusion of benefit: does a woman with breast cancer derive benefit from an intervention delivered to her partner? Oncology Nursing Forum, 38(2):207-214. doi: 10.1188/11.ONF.207214.

Cohen, M., Mabjish, A. A., \& Zidan, J. (2011). Comparison of Arab breast cancer patients and healthy controls for spousal relationship, body image, and emotional distress. Quality of Life Research, 20(2), 191-198. doi: 10.1007/s11136-010-97479.

Colon, Y. (2015). Working with sociocultural and economic diversity. In G. Christ, C. Messner, \& L. Behar (eds.), Handbook of Oncology Social Work (pp.263-268). New York, NY: Oxford University Press.

Comstock, D. L., Hammer, T. R., Strentzsch, J., Cannon, K., Parsons, J., \& Salazar II, G. (2008). Relational-cultural theory: A framework for bridging relational, multicultural, and social justice competencies. Journal of Counseling \& Development, 86, 279-287. doi:10.1002/j.1556-6678.2008.tb00510.x. 
Connell, S., Patterson, C., \& Newman, B.(2006). A qualitative analysis of reproductive issues raised by young Australian women with breast cancer. Health Care for Women International, 27(1), 94-110. doi: 10.1080/07399330500377580.

Cordova, M. J., Cunningham, L. L. C., Carlson, C. R., \& Andrykowski, M. A. (2001a). Posttraumatic growth following breast cancer: A controlled comparison study. Health Psychology, 20(3), 176-185. http://dx.doi.org/10.1037/0278-6133.20.3.176.

Cormio, C., Romito, F., Viscanti, G., Turaccio, M., Lorusso, V., \& Mattioli, V. (2014). Psychological well-being and posttraumatic growth in caregivers of cancer patients. Frontiers in Psychology, 5, 1342. doi: 10.3389/fpsyg.2014.01342.

Coyne, E., \& Borbasi, S. (2007). Holding it all together: Breast cancer and its impact on life for younger women. Contemporary Nurse, 23(2), 157-169.

Coyne, J. C., \& DeLongis, A. (1986). Going beyond social support: The role of social relationships in adaptation. Journal of Consulting Clinical Psychology, 54(4), 454460. http://dx.doi.org/10.1037/0022-006X.54.4.454.

Coyne, J. C., Ellard, J.H., \& Smith, D.A. (1990). Unsupportive relationships, interdependence, and unhelpful exchanges. In I. G. Sarason, \& Pierce, G. (Ed.), Social support: An interactional view (pp. 129-149). New York, NY: Wiley.

Coyne, J. C., \& Fiske, V. (1992). Couples coping with chronic and catastrophic. In M. A. P. Stephens, Hobfoll, S.E., \& Crowther, J. (Ed.), Family health psychology (pp. 129-149). Washington, DC: Hemisphere.

Coyne, J. C., \& Smith, D.A.F. (1991). Couples coping with a myocardial infarction: A contextual perspective on wives distress. Journal of Personality and Social Psychology, 61(3), 404-412. http://dx.doi.org/10.1037/0022-3514.61.3.404. 
Coyne, J. C., \& Smith, D.A.F. (1994). Couples coping with a myocardial infarction: A contextual perspective on patient self-efficacy. Journal of Family Psychology, 8(1), 43-54. http://dx.doi.org/10.1037/0022-3514.61.3.404.

Coyne, J.C., Thompson, R., \& Palmer, S.C. (2002). Marital quality, coping with conflict, marital complaints, and affection in couples with a depressed wife. Journal of Family Psychology, 16(1):26-37. http://dx.doi.org/10.1037/0893-3200.16.1.26.

Coyne, J. C., Wortman, C., \& Lehman, D. (1988). The other side of support: Emotional overinvolvement and miscarried helping. In B. H. Gottlieb (Ed.), Social support: Formats, processes, and effects (pp. 305-330). New York, NY: Sage.

Cozolino, L. (2006). The Neuroscience of Human Relationships: Attachment and the Developing Social Brain. New York, NY: W. W. Norton \& Co.

Crist, J.V., \& Grunfeld, E.A. (2013). Factors reported to influence fear of recurrence in cancer patients: A systematic review. Psycho-Oncology. 22(5):978-986. doi: 10.1002/pon.3114.

Cutrona, C. E. (1996a). Social support as a determinant of marital quality. In G. R. Pierce, B. R. Sarason \& I. G. Sarason (Eds.), Handbook of Social Support and the Family (pp. 173-194). New York: Plenum Press.

Cutrona, C. E. (1996b). Social Support in Couples. Thousand Oaks, CA: Sage.

D’Ardenne, P. (2004). The couple sharing long-term illness. Sexual and Relationship Therapy, 19(3), 291-308. doi: 10.1080/14681990410001715427.

Damaskos, P., \& Parry, C. (2015). Cancer Survivorship: Concepts, Interventions, and Research. In G. Christ, C. Messner, \& L. Behar (eds.), Handbook of Oncology Social Work (pp. 57-64). New York, NY: Oxford University Press. 
Danhauer, S.C., Crawford, S.L., Farmer, D.F., \& Avis, N.E. (2009). A longitudinal investigation of coping staregies and quality of life among younger women with breast cancer. Journal of Behavioral Medicine, 32(4), 371-379. doi: 10.1007/s10865-009-9211-x.

Davila, J., Karney, B.R., \& Bradbury, T.N. (1999). Attachment change processes in the early years of marriage. Journal of Personality and Social Psychology, 76(5), 783802. http://dx.doi.org/10.1037/0022-3514.76.5.783.

de Groot, J. M., Mah, K., Fyles, A., Winton, S., Greenwood, S., Depetrillo, A.D., \& Devins, G.M. (2005). The psychosocial impact of cervical cancer among affected women and their partners. International Journal of Gynecological Cancer, 15(5), 918-925. doi: 10.1111/j.1525-1438.2005.00155.x.

de Groot, J. M., Mah, K., Fyles, A., Winton, S., Greenwood, S., DePetrillo, D., \& Devins, G. M. (2007). Do single and partnered women with gynecologic cancer differ in types and intensities of illness- and treatment-related psychosocial concerns? A pilot study. Journal of Psychosomatic Research, 63(3), 241-245. doi: 10.1016/j.jpsychores.2007.02.011

Dehle, C., Larsen, D., \& Landers, J. (2001). Social support in marriage. The American Journal of Family Therapy, 29(4), 307-324. doi:10.1080/01926180126500.

DeLongis, A., \& Holtzman, S. (2005). Coping in context:the role of stress, social support, and personality in coping. Journal of Personality, 73(6), 1633-1656. doi:10.1111/j.1467-6494.2005.00361.x.

DeLongis, A., \& O'Brien, T. B. (1990). An interpersonal framework for stress and coping: An application to the families of Alzheimer's patients. In M. A. P. Stephens, 
J. H. Crowther, S. E. Hobfoll \& D. L. Tennenbaum (Eds.), Stress and Coping in Later Life Families (pp. 221-239). Washington, DC: Hemisphere.

Dember, W. N. (1974). Motivation and the cognitive revolution. American Psychologist, 29(3), 161-168. doi: http://dx.doi.org/10.1037/h0035907.

Den Oudsten, B. L., Traa, M.J., Thong, M.S.Y., Martijn, H., De Hingh, I.H., Bosscha, K., \& van de Poll-Franse, L.V. (2012). Higher prevalence of sexual dysfunction in colon and rectal cancer survivors compared with the normative population: A population-based study. European Journal of Cancer, 48(17), 3161-3170. doi: 10.1016/j.ejca.2012.04.004.

Den Oudsten, B. L., Van Heck, G. L., Van der Steeg, A. F., Roukema, J. A., \& De Vries, J. (2010). Clinical factors are not the best predictors of quality of sexual life and sexual functioning in women with early stage breast cancer. Psycho-Oncology, 19(6), 646-656. doi: 10.1002/pon.1610

DeSantis, C., Ma, J., Bryan, L., \& Jemal, A. (2014). Breast Cancer Statistics, 2013. CA: A Cancer Journal for Clinicians, 64, 52-62.

Deshields, T.L., Rihanek, A., Potter, P., Zhang, Q., Kuhrik, M., Kuhrik, N., \& O’Neill, J. (2012). Psychosocial aspects of caregiving: perceptions of cancer patients and family caregivers. Supportive Care in Cancer, 20(2), 349-356. doi:

10.1007/s00520-011-1092-1.

Devins, G.M. (1994). Illness intrusiveness and the psychosocial impact of lifestyle disruptions in chronic life-threatening disease. Advances in Renal Replacement Therapy, 1(3), 251-263. 
Devins, G.M. (2010). Using the Illness Intrusiveness Ratings Scale to understand healthrelated quality of life in chronic disease. Journal of Psychosomatic Research, 68(6), 591-602. doi:10.1016/j.jpsychores.2009.05.006.

Devins, G.M., Binik, Y.M., Hutchinson, T.A., et al (1983). The emotional impact of endstage renal disease: Importance of patients' perceptions of intrusiveness and control. International Journal of Psychiatry in Medicine, 13(4), 327-343.

Devins, G.M., Hunsley, J., Mandin, H., Taub, K.J., \& Paul, LC. (1997). The marital context of end-stage renal disease: Illness intrusiveness and perceived changes in family environment. Annals of Behavioral Medicine, 19(4), 325-332.

Devins, G.M., Mandin, H., Hons, R.B., et al (1990). Illness intrusiveness and quality-oflife in end-stage renal disease: Comparison and stability across treatment modalities. Health Psychology, 9(2), 117-142. doi: 10.1037/0278-6133.9.2.117.

Diehl, M., Chui, H., Hay, E. L., Lumley, M. A., Grühn, D., \& Labouvie-Vief, G. (2014). Change in coping and defense mechanisms across adulthood: Longitudinal findings in a European-American Sample. Developmental Psychology, 50(2), 634-648. http://doi.org/10.1037/a0033619.

Diehl, M., Coyle, N., \& Labouvie-Vief, G. (1996). Age and sex differences in strategies of coping and defense across the life span. Psychology and Aging. 11, 127-139. doi: 10.1037/0882-7974.11.1.127.

DiSipio, T., Hayes, S., Newman, B., \& Janda, M. (2008). Health-related quality of life 18 months after breast cancer: Comparison with the general population of Queensland, Australia. Supportive Care in Cancer. 16(10), 1141-1150. doi: 10.1007/s00520007-0392-y. 
Dixon, R. A., \& Gould, O. N. (1996). Adults telling and retelling stories collaboratively. In P. B. Baltes \& U. M. Staudinger (Eds.), Interactive minds: Life-span perspectives on the social foundation of cognition (pp. 221-241). New York: Cambridge University Press.

Donato, S. (2012). Il coping diadico, ovvero far fronte allo stress insieme: Una rassegna della letteratura [Dyadic coping, that is managing stress together: A review of the literature]. Giornale Italiano di Psicologia, 3, 473-504, doi: 10.1421/78499.

Donato, S., Iafrate, R., \& Barni, D. (2013). Parents as models for partners' relational competneces: Theoretical Bases and Empirical Findings. In P. Barberis \& S. Petrakis (eds.). Parenting Challenges, Practices and Cultural Influences (83-102). Hauppauge, NY: Nova Science Publishers.

Donato, S., Iafrate, R., Bradbury, T.N., \& Scabini, E. (2012). Acquiring dyadic coping: Parents and partners as models. Personal Relationships, 19(2), 386-400. doi:10.1111/j.1475-6811.2011.01368.x.

Donato, S., \& Parise, A. (2011). The role of enacted and perceived dyadic coping for young couples' satisfaction. In B. Molinelli, \& V. Grimaldo (Eds.). Handbook of the Psychology of Coping: New Research (pp.261-278). Hauppauge, NY: Nova Science Publisher.

Dorros, S. M., Card, N.A., Segrin, C., \& Badger, T.A. (2010). Interdependence in women with breast cancer and their partners: An interindividual model of distress. Journal of Consulting and Clinical Psychology, 78(1), 121-125. 10.1037/a0017724

Dorval, M., Guay, S., Mondor, M., Masse, B., Falardeau, M., Robidoux, A., \& Maunsell, E. (2005). Couples who get closer after breast cancer: Frequency and predictors in a 
prospective investigation. Journal of Clinical Oncology, 23(15), 3588-3596. doi: 10.1200/JCO.2005.01.628

Dow, K.H., \& Kuhn, D. (2004). Fertility options in young breast cancer survivors: A review of the literature. Oncology Nursing Forum, 31(3), E46-E53. doi: 10.1188/04.ONF.E46-E53.

Drabe, N., Wittman, L., Zwahlen, D., Buchi, S., \& Jenewein, J. (2013). Changes in close relationships between cancer patients and their partners Psycho-Oncology, 22(6), 1344-52. doi: 10.1002/pon.3144.

Duffy, C.M., Allen, S.M., \& Clark, M.A. (2005). Discussions regarding reproductive health for young women with breast cancer undergoing chemotherapy. Journal of Clinical Oncology, 23(4), 766-773. doi: 10.1200/JCO.2005.01.134.

Duggleby, W., Doell, H., Cooper, D., Thomas, R., \& Ghosh, S. (2014). The quality of life of male spouses of women with breast cancer: Hope, self-efficacy, and perceptions of guilt. Cancer Nursing, 37(1), E28-E35. doi: 10.1097/NCC.0b013e31827ca807.

Dumont, S., Turgeon, J., Allard, P., Gagnon, P., Charbonneau, C., \& Vezina, L. (2006). Caring for a loved one with advanced cancer: Determinants of psychological distress in family caregivers. Journal of Palliative Medicine, 9, 912-921. doi:10.1089/jpm.2006.9.912.

Dunn, J., \& Steginga, S. (2000). Young women's experience of breast cancer: Defining young and identifying concerns. Psycho-Oncology, 9(2), 137-146. doi: 10.1002/(SICI)1099-1611(200003/04)9:2<137::AID-PON442>3.0.CO;2-0

Duvall, E. M. (1957). Family Development. Philadelphia, PA: J.B. Lippincott 
Duvall, E.M., \& Hill, R.L.. (1948). Reports of the committee on the dyanamics of family interaction. Washington, D.C. : National Conference on Family Life

Duvall, E.M., \& Miller, B.C. (1985). Marriage and family development. 6th edition. New York, NY: Harper and Row

Easley, J., Miedema, B., \& Robinson, L. (2013). It's the "Good" cancer, so who cares?

Perceived lack of support among young thyroid cancer survivors. Oncology Nursing Forum, 40(6), 596-600. doi: 10.1188/13.ONF.596-600.

Ebbesen, L.S., Guyatt, G.H.,MCCartney, N., \& Oldridge, N.B. (1990). Measuring quality of life in cardiac spouses. Journal of Clinical Epidemiology, 43(5), 481-487. doi: http://dx.doi.org/10.1016/0895-4356(90)90137-E.

Edwards, B., \& Ung, L. (2002). Quality of life instruments for caregivers of patients with cancer: A review of their psychometric properties. Cancer Nursing, 25(5), 342-349.

Elder, G. H., Jr. (1998). The life course as developmental theory. Child Development, 69(1), 1-12. doi: http://dx.doi.org/10.2307/1132065.

Elder, G. H., Jr. (2000). Life course theory. [Encyclopedia Entry]. In A.E. Kazdin (Ed.), Encyclopedia of psychology, Vol. 5, (pp. 50-52). Washington DC: American Psychological Association. doi: http://dx.doi.org/10.1037/10520-020.

Elder Jr., G. H. (2002). The life course and aging: Some accomplishments, unfinished tasks, and new directions. Paper presented at the Annual Meeting of the Gerontological Society of America, Boston, Massachusetts.

Elder, G.H., Jr., Johnson, M.K., \& Crosnoe, R. (2006). The emergence and development of Life Course Theory. In J.T. Mortimer and M.J. Shanahan (Eds.). Handbook of the Life Course (pp.3-19). New York, NY: Springer. 
Elder, G. H., Jr., \& Shanahan, M. J. (2006). The life course and human development. In R. M. Lerner \& W. Damon (Eds.), Handbook of child psychology: Theoretical models of human development (Vol. 1, pp. 665-715). Hoboken, NJ: John Wiley \& Sons Inc.

Elk, R. \& Landrine, H. (Eds) (2012). Cancer disparities: causes and evidence-based solutions. NewYork, NY: Springer Publishing Company.

Ell, K., Nishimoto, R., Mantel, J., \& Harmovitch, M. (1988). A longitudinal analysis of psychological adaptation among family members of patients with cancer. Journal of Psychosomatic Research, 32(4), 429-438. doi: http://dx.doi.org/10.1016/00223999(88)90026-8.

Ell, K., Nishimoto, R., Morvay, T., Mantell, J., \& Harmovitch, M. (1996). A longitudinal analysis of psychological adaptation among survivors of cancer. Cancer, 63(2), 406-413. doi: 10.1002/1097-0142(19890115)63:2<406::aidcncr2820630234>3.0.co;2-2.

Ellis, A. (1957). Outcome of employing three techniques of psychotherapy. Journal of Clinical Psychology, 13, 344-350. doi: 10.1002/1097-4679(195710)13:4<344::aidjclp2270130407>3.0.co;2-9.

Engel, J., Kerr, J., Schlesinger-Raab, A., Sauer, H., \& Holzel, D. (2004) Quality of life following breast-conserving therapy or mastectomy: results of a 5-year prospective study. Breast Journal, 10(3), 223-231. doi:10.1111/j.1075-122X.2004.21323.x.

Eton, D. T., Lepore, S.J., \& Helgeson, V.S. (2005). Psychological distress in spouses of men treated for early-stage prostate carcinoma. Cancer, 103(11), 2412-2418. doi: 10.1002/cncr.21092. 
Fagundes, C.P., Berg, C.A., \& Wiebe, D.J. (2012). Intrusion, avoidance, and daily negative affect among couples coping with prostate cancer: a dyadic investigation. Journal of Family Psychology, 26(2), 246-253. doi: 10.1037/a0027332

Fairbairn, W. R. D. (1952). Psychological Studies of the Personality. London: Routledge \& Kegan Paul.

Fairbairn, W. R. D. (1954). Observations on the nature of hysterical states. British Journal of Medical Psychology, 27, 105-125.

Fairbairn, W. R. D. (1958). On the nature and aims of psychoanalytic treatment. International Journal of Psychoanalyisis., 39(5), 374-385.

Falconier, M.K., Jackson, J.B., Hilpert, P., \& Bodenmann, G. (2015). Dyadic coping and relationship satisfaction: A meta-analysis. Clinical Psychology Review, 42, 28-46. doi: 10.1016/j.cpr.2015.07.002.

Falconier, M. K., Nussbeck, F., \& Bodenmann, G. (2013). Dyadic coping in Latino couples: Validity of the Spanish version of the Dyadic Coping Inventory. Anxiety, Stress, \& Coping, 26, 446-466.doi: 10.1080/10615806.2012.699045

Falconier, M. K., Nussbeck, F., \& Bodenmann, G. (2013). Immigration stress and relationship satisfaction in Latino couples: The role of dyadic coping. Journal of Social and Clinical Psychology, 32(8), 813-843. doi: 10.1521/jscp.2013.32.8.813.

Fang, C. Y, Manne, S. L., \& Pape, S. J. (2001). Functional impairment, marital quality, and patient psychological distress as predictors of psychological distress among cancer patients' spouses. Health Psychology, 20(6), 452-457. doi: 10.1037/02786133.20.6.452. 
Faul, F., Erdfelder, E., Buchner, A., \& Lang, A.-G. (2009). Statistical power analyses using G*Power 3.1: Tests for correlation and regression analyses. Behavior Research Methods, 41, 1149-1160.

Faul, F., Erdfelder, E., Lang, A.-G., \& Buchner, A. (2007). G*Power 3: A flexible statistical power analysis program for the social, behavioral, and biomedical sciences. Behavior Research Methods, 39, 175-191.

Fekete, E. M., Parris Stephens, M.A., Mickelson, K.D., \& Druley, J.A. (2007). Couples' Support Provision during Illness: The role of perceived emotional responsiveness. Families, Systems, \& Health, 25(2), 204-217. http://dx.doi.org/10.1037/10917527.25.2.204.

Feldman, B. N., \& Broussard, C. A. (2005). The influence of relational factors on men's adjustment to their partners' newly diagnosed breast cancer. Journal of Psychosocial Oncology, 23(2-3), 23-43. doi: 10.1300/J077v23n02_03

Feldman, B.N., \& Broussard, C.A. (2006). Men's adjustment to their partners' breast cancer: a dyadic coping perspective. Health \& Social Work, 31(2), 117-127. doi: 10.1093/hsw/31.2.117.

Felton, B. J., \& Revenson, T. A. (1987). Age differences in coping with chronic illness. Psychology and Aging, 2(2), 164-170. http://dx.doi.org/10.1037/0882-7974.2.2.164.

Fenn, K. M., Evans, S. B., McCorkle, R., DiGiovanna, M. P., Pusztai, L., Sanft, T., Hofstatter, E. W., Killelea, B. K., Knobf, M. T., Lannie, D. R., Abu-Khalaf, M., Horowitz, N. R., \& Chagpar, A. B. (2014). Impact of financial burden of cancer on survivors' quality of life. Journal of Oncology Practice/American Society of Clinical Oncology, 10(5), 332-338. doi: 10.1200/JOP.2013.001322. 
Fergus, K., Ahmad, S., McLeod, D.L., Stephen, J., Gardner, S., Pereira, A., Warner, E., \& Carter, W. (2015). Couplelinks- An online intervention for young women with breast cancer and their male partner: Study protocol for a randomized controlled trial. Trials, 16, 33. doi:10.1186/s13063-014-0534-8.

Fergus, K. D., \& Gray, R. E. (2009). Relationship vulnerabilities during breast cancer: Patient and partner perspectives. Psycho-Oncology, 18(12), 1311-1322. doi:10.1002/pon.1555.

Fergus, K.D., McLeod, D., Carter, W., Warner, E., Gardner, S., Granek, L. et al. (2014). Development and pilot testing of an online intervention to support young couples' coping and adjustment to breast cancer. European Journal of Cancer Care, 23(4), 481-492. doi:10.1111/ecc.12162.

Fife, B.L.,Weaver, M.T.,Cook, W. L.; Stump, T. T. (2013). Partner interdependence and coping with life-threatening illness: The impact on dyadic adjustment. Journal of Family Psychology, 27(5), 702-711. doi.org/10.1037/a0033871.

Fingerman, K.L., Berg, C.A., Smith, J., \& Antonucci, T.C. (2001). Handbook of LifeSpan Development. New York: Springer Publishing.

Fingerman, K.L., Hay, E.L., \& Birditt, K.S. (2004). The best of ties, the worst of ties: Close, problematic, and ambivalent social relationships. Journal of Marriage and the Family, 66(3), 792-808. doi:10.1111/j.0022-2445.2004.00053.x.

Fischer, M., Baucom, B.R., Weusthoff, S., Atkins, D.A., Baucom, D.H., Hahlweg, K., \& Zimmerman, T. (2015). Social support behavior and encoded emotional arousal during couple communication about breast cancer. Journal of Family Psychology, 29(5), 744-754. doi: 10.1037/fam0000092. 
Fitch, M. I., \& Allard, M. (2007). Perspectives of husbands of women with breast cancer: Impact and response. Canadian Oncology Nursing 17(2), 66-78. doi: $10.5737 / 1181912 \times 1726671$.

Fitzell A, \& Pakenham, K.I.(2010). Application of a stress and coping model to positive and negative adjustment outcome in colorectal cancer caregiving. PsychoOncology. 19(11), 1171-1178. doi: 10.1002/pon.1666.

Fletcher, B.S., Miaskowski, C., Given, B., \& Schumacher, K. (2012). The cancer family caregiving experience: An updated and expanded conceptual model. European Journal of Oncol Nursing, 16(4), 387-398. doi: 10.1016/j.ejon.2011.09.001.

Flynn, K. E., Jeffery, D. D., Keefe, F. J., Porter, L. S., Shelby, R. A., Fawzy, M. R., \& Weinfurt, K. P. (2011). Sexual functioning along the cancer continuum: Focus group results from the Patient-Reported Outcomes Measurement Information System (PROMIS®). Psycho-Oncology, 20(4), 378-386. doi: 10.1002/pon.1738.

Fobair, P., Stewart, S.L., Chang, S., D’Onofrio, C., Banks, P., \& Bloom, J.R. (2006). Body image and sexual problems in young women with breast cancer. PsychoOncology, 15(7), 579-594. doi: 10.1002/pon.991.

Folkman, S. (1984). Personal control and stress and coping processes: A theoretical analysis. Journal of Personality and Social Psychology,48(6), 839-852.

Folkman, S. (1997). Positive psychological states and coping with severe stress. Social Science and Medicine, 45(8), 1207-1221. doi:10.1016/S0277-9536(97)00040-3. Folkman, S. (2011). The Oxford Handbook of Stress, Health and Coping. New York, NY: Oxford University Press. 
Folkman, S., \& Greer, S. (2000). Promoting psychological well-being in the face of serious illness; when theory, research and practice inform each other. PsychoOncology, 9(1), 11-19. doi: 10.1002/(sici)1099-1611(200001/02)9:1<11::aidpon424>3.0.co;2-z.

Folkman, S. \& Lazarus, R. S. (1980). An analysis of coping in a middle-aged community sample. Journal of Health and Social Behavior,21, 219- 239. http://www.jstor.org/stable/2136617.

Folkman, S. \& Lazarus, R. S. (1985). If it changes it must be a process: Study of emotion and coping during three stages of a college examination. Journal of Personality and Social Psychology,48, 150-170. http://dx.doi.org/10.1037/0022-3514.48.1.150.

Folkman, S. \& Lazarus, R. S. (1988). Coping as a mediator of emotion. Journal of Personality and Social Psychology, 54, 466-475. http://dx.doi.org/10.1037/00223514.54.3.466.

Folkman, S., Lazarus, R. S., Dunkel-Schetter, C., DeLongis, A. and Gruen, R. J. (1986). Dynamics of a stressful encounter: cognitive appraisal, coping, and encounter outcomes. Journal of Personality and Social Psychology, 50(5), 992-1003. http://dx.doi.org/10.1037/0022-3514.50.5.992.

Folkman, S., Lazarus, R.S., Gruen, R., \& DeLongis, A. (1986). Appraisal, coping, health status, and psychological symptoms. Journal of Personality and Social Psychology, 50(3), 571-579. http://dx.doi.org/10.1037/0022-3514.50.3.571.

Folkman S., Lazarus R.S., Pimley S, \& Novacek J. (1987). Age differences in stress and coping processes. Psychology \& Aging, 2(2), 171-184.

http://dx.doi.org/10.1037/0882-7974.2.2.171. 
Folkman, S., \& Moskowitz, J. T. (2000a). The context matters. Personality and Social Psychology Bulletin, 26(2), 150-151. doi: 10.1177/0146167200264002.

Folkman, S., \& Moskowitz, J. T. (2000b). Positive affect and the other side of coping. American Psychologist, 55(6), 647-654. http://dx.doi.org/10.1037/0003066X.55.6.647.

Folkman, S.\& Moskowitz, J.T.(2004).Coping: Pitfalls and promise. Annual Review of Psychology, 55, 745-774. doi: 10.1146/annurev.psych.55.090902.141456.

Fredman, S.J., Baucom, D.H., Gremore, T.M., Castellani, A.M., Kallman, T.A, Porter, L.S., et al. (2009). Quantifying the recruitment challenges with couple-based interventions for cancer: applications to early-stage breast cancer. PsychoOncology, 18(6),667-673. doi: 10.1002/pon.1477.

Freedberg, S. (2009). Relational theory for social work practice: A feminist perspective. NY: Routledge Press.

Freedberg, S. (2015). Relational Theory for Clinical Practice. New York, NY: Routledge.

Freeman, S. J. (2000). Ethics. An Introduction to Philosophy \& Practice. Belmont, CA: Wadsworth.

French, J. P. P., Jr., Rodgers, W., \& Cobb, S. (1974). Adjustment as a personenvironment fit. In G. V. Coelho, Hamburg, D.A., \& Adams, J.E. (Ed.), Coping and adjustment (pp. 316-333). New York: NY: Basic Books

Fu, M. R., Xu, B., Liu, Y., \& Haber, J. (2008). Making the best of it: Chinese women's experiences of adjusting to breast cancer diagnosis and treatment. Journal of Advanced Nursing, 63(2), 155-165. doi: 10.1111/j.1365-2648.2008.04647.x. 
Galbraith, M. E., Pedro, L.W., Jaffe, A.R., \& Allen, T.L. (2008). Describing healthrelated outcomes for couples experiencing prostate cancer: Differences and similarities. Oncology Nursing Forum, 35(5), 794-801. doi: 10.1188/08.ONF.794801.

Ganz, P.A. (2010). Quality-of-life issues in patients with ductal carcinoma in situ. Journal of the National Cancer Institute Monographs, 41, 218-222. doi: 10.1093/jncimonographs/lgq029.

Ganz, P.A., Desmond, K.A., Leedham, B, Rowland, J.H., Meyerowitz, B.E., \& Belin, T.R. (2002). Quality of life in long-term, disease-free survivors of breast cancer: A follow-up study. Journal of the National Cancer Institute, 94(1), 39-49. doi: 10.1093/jnci/94.1.39.

Ganz, P.A., Greendale, G.A., Petersen, L, Zibecchi, L., Kahn, B., \& Belin, T.R. (2000). Managing menopausal symptoms in breast cancer survivors: Results of a randomized controlled trial. Journal of the National Cancer Institute, 92,10541064. doi: 10.1093/jnci/92.13.1054.

Ganz, P.A., Greendale, G.A., Petersen, L., Kahn, B., \& Bower, J,E. (2003). Breast cancer in younger women: Reproductive and late health effects of treatment. Journal of Clinical Oncology, 21(22), 4184-93. doi: 10.1200/JCO.2003.04.196 .

Ganz, P.A., Rowland, J.H., Desmond, K., Meyerowitz, B.E., \& Wyatt, G.E. (1998). Life after breast cancer: Understanding women's health-related quality of life and sexual functioning. Journal of Clinical Oncology, 16(5),501-514. 
Gehlert, S., \& Coleman, R. (2010). Using Community-Based Participatory Research to ameliorate cancer disparities. Health \& Social Work, 35(4), 302-309.doi: 10.1093/hsw/35.4.302.

Genero, N.P., Baker Miller, J., \& Surrey, J. (1990). Project Report: The Mutual Psychological Development Questionnaire. Wellesley, MA: Stone Center at Wellesley College.

Genero, N.P., Baker Miller, J., Surrey, J., \& Baldwin, L.M. (1992). Measuring perceived mutuality in close relationships: Validation of the Mutual Psychological Development Questionnaire. Journal of Family Psychology, 6(1), 36-48. http://dx.doi.org/10.1037/0893-3200.6.1.36.

Germino, B. B., Fife, B.L., \& Funk, S.G. (1995). Cancer and the partner relationship: what is its meaning? Seminars in Oncology Nursing, 11(1), 43-50. doi: http://dx.doi.org/10.1016/S0749-2081(95)80042-5.

Geue, K., Schmidt, R., Sender, A., Sauter, S., \& Friedrich, M. (2015). Sexuality and romantic relationships in young adult cancer survivors: Satisfaction and supportive care needs . Psycho-Oncology, 24(11), 1368-1376. doi: 10.1002/pon.3805. Epub 2015 Mar 31.

Giannousi, Z., Karademas, E.C., \& Dimitraki, G. (2016). Illness representations and psychological adjustment of Greek couples dealing with a recently-diagnosed cancer: dyadic, interaction and perception-dissimilarity effects. Journal of Behavioral Medicine, 39(1), 85-93. doi: 10.1007/s10865-015-9664-z. 
Giese-Davis, J., Hermanson, K., Koopman, C., Weibel, D., \& Spiegel C. (2000). Quality of couples relationship and adjustment to metastatic breast cancer. Journal of Family Psychology, 14(2), 251-266. doi: 10.1037/0893-3200.14.2.251.

Gilbert, E., Ussher, J. M., \& Perz, J. (2010). Re-negotiating sexuality and intimacy postcancer: The experiences of carers in a couple relationship with a person with cancer. Archives of Sexual Behavior, 39(4), 998-1109. doi: 10.1007/s10508-0089416-z.

Gilbert, E., Ussher, J. M., \& Perz, J. (2011). Sexuality after gynaecological cancer: a review of the material, intrapsychic, and discursive aspects of treatment on women's sexual-wellbeing. Maturitas, 70(1), 42-57. doi: 10.1016/j.maturitas.2011.06.013

Gilligan, C. (1982). In a different voice. Cambridge, MA: Harvard University Press.

Godwin, K.M., Swank, P.R., Vaeth, P., \& Ostwald, S.K. (2013). The longitudinal and dyadic effects of mutuality on perceived stress for stroke survivors and their spousal caregivers. Aging \& Mental Health, 17(4), 423-431.

doi:10.1080/13607863.2012.756457.

Gold, M., Dunn, L.B., Phoenix, B, Paul, S.M., Hamolsky, D., Levine, J.D., \& Miaskowski, C. (2015). Co-occurrence of anxiety and depressive symptoms following breast cancer surgery and its impact on quality of life. European Journal of Oncology Nursing, Jul 15. doi: 10.1016/j.ejon.2015.06.003.

Gomez-Campelo, P., Bragado-Alvarez, C., \& Hernandez-Lloreda, M.J. (2014).

Psychological distress in women with breast and gynecological cancer treated with radical surgey. Psycho-Oncology, 23(4), 459-466. doi:10.1002/pon.3439. 
Goodman, S.A. \& Svyantek, D.J. (1999). Person-organization fit and contextual performance: Do shared values matter?. Journal of Vocational Behavior, 55, 254275. doi: $10.1006 /$ jvbe.1998.1682.

Gorman, J. R., Malcarne, V. L., Roesch, S. C., Madlensky, L., \& Pierce, J. P. (2010). Depressive symptoms among young breast cancer survivors: the importance of reproductive concerns. Breast Cancer Research \& Treatment, 123(2), 477-485. doi: $10.1007 / \mathrm{s} 10549-010-0768-4$

Gorman, J.R., Su, H.I., Pierce, J.P., Roberts, S.C., Dominick, S.A., \& Malcarne, V.L. (2014). A multidimensional scale to measure the reproductive concerns of young adult female cancer survivors. Journal of Cancer Survivorship, 8(2), 218-228. doi: 10.1007/s11764-013-0333-3.

Gorman, J.R., Usita, P.M., Madlensky, L., \& Pierce, J.P. (2011). Young breast cancer survivors: Their perspectives on treatment decisions and fertility concerns. Cancer Nursing, 34(1), 32-40. doi: 10.1097/NCC.0b013e3181e4528d.

Götze, H., Brähler, E., Gansera, L., Polze, N., \& Köhler, N. (2014). Psychological distress and quality of life of palliative cancer patients and their caring relatives during home care. Supportive Care in Cancer, 22(10):2775-82. doi: $10.1007 / \mathrm{s} 00520-014-2257-5$

Graca Pereira, M., Figueiredo, A.P., \& Fincham, F.D. (2012). Anxiety, depression, traumatic stress and quality of life in colorectal cancer after different treatments: A study with Portuguese patients and their partners. European Journal of Oncology Nursing, 16(3):227-232. doi: 10.1016/j.ejon.2011.06.006. 
Gross, J., Carstensen, L. L., Pasupathi, M. Tsai, J., Götestam Skorpen, C., \& Hsu, A. (1997). Emotion and aging: Experience, expression and control. Psychology and Aging, 12, 590-599. http://dx.doi.org/10.1037/0882-7974.12.4.590.

Grunfeld, E., Coyle, D., Whelan, T., Clinch, J., Reyno, L., Earle, C.C., Willan, A., Viola, R., Coristine, M., Janz, T., \& Glossop, R. (2004). Family caregiver burden: Results of a longitudinal study of breast cancer patients and their principal caregivers. Canadian Medical Association Journal, 170(12), 1795-1801. doi: 10.1503/cmaj.1031205.

Gurevich, M., Bishop, S., Bower, J., Malka, M.,\& Nyhof-Young, J. (2004).

(Dis)embodying gender and sexuality in testicular cancer. Social Science \& Medicine, 58(9), 1597-1607. doi:10.1016/s0277-9536(03)00371-x.

Gurman, A. S., \& Kniskern, D.P. (2003). Manuale di Terapia della Famiglia. Torino: Bollati Boringhieri.

Gurung, R.A., Taylor, S.E., \& Seeman, T.E. (2003). Accounting for changes in social support among married older adults: insights from the MacArthur Studies of Successful Aging. Psychology \& Aging. 18(3), 487-496. http://dx.doi.org/10.1037/0882-7974.18.3.487.

Hagestad, G.O. (1988). Demographic change and the life course: some emerging trends in the family realm. Family Relations, 37(4), 405-410 .

Hadden, B. W., Smith, C. V., \& Webster, G. D. (2014). Relationship duration moderates associations between attachment and relationship quality: Meta-analytic support for the temporal adult romantic attachment model. Personality and Social Psychology Review, 18, 42-58. doi:10.1177/1088868313501885. 
Hagedoorn, M., Buunk, B. P., Kuijer, R. G., Wobbes, T., \& Sanderman, R. (2000).

Couples dealing with cancer: Role and gender differences regarding psychological distress and quality of life. Psycho-Oncology, 9(3), 232-242. doi:10.1002/10991611(200005/06)9:3<232::AID-PON458>3.0.CO;2-J

Hagedoorn, M., Dagan, M., Puterman, E., Hoff, C., Meijerink, W. J., Delongis, A., \& Sanderman, R.(2011). Relationship satisfaction in couples confronted with colorectal cancer: The interplay of past and current spousal support. Journal of Behavioral Medicine, 34, 288-297. doi:10.1007/s10865-010-9311-7

Hagedoorn, M., Hein, F. L., Schulz, T., van der Heide, J. J. H., Niesing, J., Westerhuis, R., \& Ranchor, A. V. (2015). Are patient and relationship variables associated with participation of intimate partners in couples research? Health Psychology, 34(3), 270-273. 10.1037/hea0000141.

Hagedoorn, M., Kuijer, R. G., Buunk, B. P., DeJong, G. M., Wobbes, T., \& Sanderman, R. (2000). Marital satisfaction in patients with cancer: Does support from intimate partners benefit those who need it the most? Health Psychology, 19, 274-282. doi:10.10371/0278-6133.19.3.274.

Hagedoorn, M., Puterman, E, Sanderman, R., Wiggers, T., Baas, P.C., van Haastert, M., \& DeLongis, A. (2011). Is self-disclosure in couples coping with cancer associated improvement in depressive symptoms? Health Psychology, 30(6), 753-762. doi: 10.1037/a0024374.

Hagedoorn, M., Sanderman, R., Bolks, H., Tuinstra, J., \& Coyne, J.C. (2008). Distress in couples coping with cancer: A meta-analysis and critical review of role and gender effects. Psychological Bulletin, 134(1), 1-30. doi: 10.1037/0033-2909.134.1.1. 
Hagestad, G. (1988). Demographic change and the life course: Some emerging trends in the family realm. Family Relations, 37, 405-410.

Haggan, P. (2002). Family resilience through sports:The family as a team. Journal of Individual Psychology, 58, 279-289.

Haid, M.L., \& Seiffge-Krenke, I. (2012). Effects of (un)employment on young couples' health and life satisfaction. Psychology \& Health, 28(3):284-301. doi: 10.1080/08870446.2012.720983.

Halliday Hardie, J. \& Lucas, A. (2010). Economic factors and relationship quality among young couples: Comparing cohabitation and marriage. Journal of Marriage and Family, 72, 1141-1154. doi: 10.1111/j.1741-3737.2010.00755.x

Hannum, J. W., Giese-Davis, J., Harding, K., \& Hatfield, A. K. (1991). Effects of individual and marital variables on coping with cancer. Journal of Psychosocial Oncology, 9(2), 1-20 . doi:10.1300/j077v09n02_01.

Harden, J.K., Sanda, M.G., Wei, J.T., Yarandi, H., Hembroff, L., Hardy, J., \& Northouse, L.L., \& PROSTQA Consortium Study Group (2013). Partners’ long-term appraisal of their caregiving experience, marital satisfaction, sexual satisfaction, and quality of life 2 years after prostate cancer treatment. Cancer Nursing, 36(2), 104-113. doi:10.1097/NCC.0b013e3182567c03.

Harden, J., Northouse, L., Cimprich, B., Pohl, J.M., Liang, J., \& Kershaw, T. (2013). The influence of developmental life stage on quality of life in survivors of prostate cancer and their partners. Journal of Cancer Survivorship, 2(2), 84-94. doi:10.1007/s 11764-008-0048-Z. 
Hartl, K., Schennach, R., Muller, M., Engel, J., Reinecker, H., Sommer, H., \& Friese, K. (2010). Quality of life, anxiety, and oncological factors: a follow-up study of breast cancer patients. Psychosomatics, 51(2), 112-123. doi:10.1176/appi.psy.51.2.112.

Hasson-Ohayon, I., Goldzweig, G., Dorfman, C., \& Uziely, B. (2014). Hope and social support utilisation among different age groups of women with breast cancer and their spouses. Psychology \& Health, 29(11), 1303-1319.

doi:10.1080/08870446.2014.929686.

Hau, E., Browne, L., Capp, A., Delaney, G.P., Fox, C., Kearsley, J.H., Millar. E., Nasser, E.H., Papadatos, G., \& Graham, P.H. (2013). The impact of breast cosmetic and functional outcomes on quality of life: long-term results from the St. George and Wollongong randomized breast boost trial. Breast Cancer Research \& Treatment, 139(1), 115-123. doi: 10.1007/s10549-013-2508-Z.

Hawkins, Y., Ussher, J., Gilbert, E., Perz, J., Sandoval, M., \& Sundquist, K. (2009). Changes in sexuality and intimacy after the diagnosis and treatment of cancer: The experience of partners in a sexual relationship with a person with cancer. Cancer Nursing, 32(4), 271-280. doi: 10.1097/NCC.0b013e31819b5a93.

Hayes, A.F. (2009). Beyond Baron and Kenny: Statistical mediation analysis in the new millennium. Communication monographs, 76, 408-420.

Hayes, A.F. (2013). Introduction to Mediation, Moderation and Conditional Process Analysis. A Regression-Based Approach. New York, NY: Guildford Press.

Heffner, K. L., Kiecolt-Glaser, J.K., Loving, T.J., Glaser, R., \& Malarkey, W.B. (2004). Spousal support satisfaction as a modifier of physiological responses to marital 
conflict in younger and older couples. Journal of Behavioral Medicine, 27(3), 233254.

Heins, M., Schellevis, F., Rijken, M., Donker,G., van der Hoek, L., \& Korevaar, J. (2013). Partners of cancer patients consult their GPs significantly more often with both somatic and psychosocial problems Scandinavian Journal of Primary Health Care, 31(4), 203-208.doi: 10.3109/02813432.2013.861153

Helgeson, V. S., Snyder, P., \& Seltman, H. (2004). Psychological and physical adjustment to breast cancer over 4 years: Identifying distinct trajectories of change. Health Psychology, 23, 3-15. http://dx.doi.org/10.1037/0278-6133.23.1.3.

Henselmans, I., Hagedoorn, M., Buunk, B. P.,\&Sanderman, R. (2004). Communicatie in de relatie alsvoorspeller van relatietevredenheid en depressieve symptomen van kankerpati€enten en hunintieme partners [Communication within the relationship as a predictor of marital satisfaction and depressive symptoms of both patients with cancer and their intimate partners]. Gedrag \&Gezondheid, 32, 75-85.

Herbenick, D., Reece, M., Hollub, A., Satinsky, S, \& Dodge, B. (2008). Young female breast cancer survivors: Their sexual function and interest in sexual enhancement products and services. Cancer Nursing, 31(6), 417-425. doi:

10.1097/01.NCC.0000339252.91194.6c.

Hill, R. (1986). Life cycle stages for types of single parent families: Of family development theory. Family Relations, 35, 19-30.

Hill, E.J., Erickson, J.J., Fellows, K.J., Martinengo, G., \& Allen, S.M. (2012). Work and family over the life course: Do older workers differ?. Journal of Family and Economic Issues, 35(1),1-13. http://dx.doi.org/10.1037/a0036941. 
Hill, E.K., Sandbo, S., Abramsohn, E., Makelarski, J., Wroblewski, K., Wenrich,E.R., McCoy, S., Temkin, S.M., Yamada, S.D., \& Lindau, S.T. (2011). Assessing gynecologic and breast cancer survivors' sexual health care needs. Cancer, 117(12), 2643-265. doi: 10.1002/cncr.25832.

Hinnen, C., Hagedoorn, M., Ranchor, A.V., \& Sanderman, R. (2008). Relationship satisfaction in women: A longitudinal case-control study abpout the role of breast cancer, personal assertiveness, and partners' relationship-focused coping. British Journal of Health Psychology, 13, 737-754. doi: 10.1348/135910707x252431.

Hinnen, C., Ranchor, A. V., Sanderman, R., Snijders, T. A., Hagedoorn, M., \& Coyne, J. C. (2008). Course of distress in breast cancer patients, their partners, and matched control couples. Annals of Behavioral Medicine, 36(2), 141-148. doi:10.1007/s12160-008-9061-8

Hochfilzer, J. E. (2010). Zufriedenheit in Paarbeziehungen. EineStudie zum Einfluss von dyadischem Coping, Equity und Kommunikation [Satisfaction in close relationships: A studyon the influence of dyadic coping, equity, and communication]. Saarbru“cken: Verlag Dr. Mu“ller

Hodges, L. J., Humphris, G.M., \& Macfarlane, G. (2005). A meta-analytic investigation of the relationship between the psychological distress of cancer patients and their carers. Social Science \& Medicine, 60(1), 1-12. doi:10.1016/j.socscimed.2004.04.018.

Holmberg, S. K., Scott, L. L., Alexy, W., \& Fife, B. L. (2001). Relationship issues of women with breast cancer. Cancer Nursing, 21(1), 53-60. 
Hoppmann, C.A., Coats, A.H., \& Blanchard-Fields, F. (2008). Goals and everyday problem solving: examining the link between age-related goals and problem-solving strategy use. Neuropsychology, development, and cognition. Section B, Aging, neuropsychology and cognition, 15(4), 401-423.

Hopwood, P., Haviland, J., Mills, J., Sumo, G., Bliss, J.M., \& START Trial Management Group. (2007). The impact of age and clinical factors on quality of life in early breast cancer: An analysis of 2208 women recruited to the UK START Trial (Standardization of Breast Radiotherapy Trial). The Breast, 16(3), 214-251. doi: http://dx.doi.org/10.1016/j.breast.2006.11.003.

Houldin, A. D. (2007). A qualitative study of caregivers' experiences with newly diagnosed advanced colorectal cancer. Oncology Nursing Forum, 34(2), 323-330. doi: 10.1188/07.onf.323-330.

Howard-Anderson, J., Ganz, P.A., Bower, J.E., \& Stanton, A.L.(2012). Quality of life, fertility concerns, and behavioral health outcomes in younger breast cancer survivors: a systematic review. Journal of the National Cancer Institute, 104(5), 386-405. doi: 10.1093/jnci/djr541

Howlader, N., Noone, A.M., Krapcho, M., Neyman, N., Aminou, R., Waldron, W., Altekruse, S.F., Kosary, C.L., Ruhl, J., Tatalovich, Z., Cho, H., Mariotto, A., Eisner, M.P., Lewis, D.R., Chen, H.S., Feuer, E.J., Cronin, K.A., \& Edwards, B.K. (eds). (2011). SEER Cancer Statistics Review, 1975-2008, National Cancer Institute. Bethesda, MD.

Høyer, M., Johansson, B., Nordin, K., Bergkvist, L., Ahlgren, J., Lidin-Lindqvist, A., Lambe, M., \& Lampic, C.(2011). Health-related quality of life among women with 
breast cancer - a population-based study. Acta Oncologica, 50(7), 1015-1026. doi: 10.3109/0284186X.2011.577446.

Hsu, T., Ennis, M., Hood, N., Graham, M., \& Goodwin, P.J. (2013). Quality of life in long-term breast cancer survivors. Journal of Clinical Oncology, 31, 3540-3548. doi: 10.1200/JCO.2012.48.1903.

Hubbard, G., Menzies, S., Flynn, P., Adams, S., Haseen, F., Thomas, I., Scanlon, K., Reed, L., \& Forbat, L. (2013). Relational mechanisms and psychological outcome in couples affected by breast cancer: a systematic narrative analysis of the literature. BMJ Supportive \& Palliative Care, 3(3), 309-317. doi: 10.1136/bmjspcare-2021000274.

Hughes, M. K. (2000). Sexuality and the cancer survivor: A silent coexistence. Cancer Nursing, 23(6), 477-482.

Hulvat, M.C, \& Jeruss, J.S. (2009). Maintaining fertility in young women with breast cancer. Current Treatment Options in Oncology, 10(5-6), 308-317. doi: 10.1007/s11864-010-0116-2.

Hutchison, E.D. (2008). Dimensions of Human Behavior: Person and Environment, Third Edition.Los Angeles, CA: Sage Publications.

Huyghe, E., Sui, D., Odensky, E., \& Schover, L. R. (2009). Needs assessment survey to justify establishing a reproductive health clinic at a comprehensive cancer center. Journal of Sexual Medicine, 6(1), 149-163. doi: 10.1111/j.1743-6109.2008.01005.x.

Iafrate, R., Bertoni, A., Donato, S., \& Finkenauer, C. (2012). Perceived similarity and understanding in dyadic coping among young and mature couples. Personal Relationships, 19(3), 401-419. doi: 10.1027/1016-9040/a000069. 
Iafrate, R., Bertoni, A., Margola, D., Cigoli, V., \& Acitelli, L.K. (2011). The link between perceptual congruence and couple relationship satisfaction in dyadic coping. European Psychologist, 17(1), 73-82. doi: 10.1027/1016-9040/a000069.

Iafrate, R., \& Donato, S. (2012). Coping in a relational context: The case of dyadic coping. In B. Molinelli \& V. Grimaldo, Handbook of the Psychology of Coping: New Research (pp. 11-132). Hauppauge, NY: Nova Science Publishers.

Iafrate, R., Donato, S., \& Bertoni. A. (2013). Family of origin heritage, individual wellbeing, and relationship satisfaction in young couples. In P.Barberis \& S. Petrakis (Eds.). Parenting Challenges, Practices and Cultural Influences (201212).Hauppauge, NY: Nova Science Publishers.

Institute of Medicine. (2008). Cancer Care for the Whole Patient: Meeting Psychosocial Health Needs: The National Academies Press

Institute of Medicine. (2013). Delivering High Quality Cancer Care. Charting a new course for a System in Crisis. The National Academies Press.

Irion, J.C., \& Blanchard-Fields, F. (1987). A cross-sectional comparison of adaptive coping in adulthood. Journal of Gerontology, 42, 502-504. doi: 10.1093/geronj/42.5.502.

Israel, B.A., Eng, E., Schulz, A.J., \& Parker, E.A. (2012). Methods in community-based participatory research for health. San Francisco, CA: Jossey-Bass.

Jamison K.R., W. D. K., \& Panau R.O. (1978). Psychological aspects of mastectomy: the woman's perspective. American Journal of Psychiatry, 135(4), 432-436. 
Jankowska, M. (2013). Sexual Functioning in young women in the context of breast cancer treatment. Reports of Practical Oncology and Radiotherapy, 18(4), 193-200. doi: http://dx.doi.org/10.1016/j.rpor.2013.04.032.

Jordan, J. (1986). The Meaning of Mutuality. Stone Center for Developmental Services and Studies. Wellesley, MA

Jordan, J. (1997). Women's growth in diversity. New York, NY: Gilford Press.

Jordan, J. (1997). The Relational Model is a source of empowerment for women. In M. R. Walsh (Ed.) Women, men, and gender: Ongoing debates. (pp. 373- 382). New Haven: Yale.

Jordan, J. (2000). The role of mutual empathy in relational/cultural therapy. Journal of Clinical Psychology 56(80), 1005-1016.

Jordan, V. J. (2009). Relational-Cultural Therapy. Washington, DC: American Psychological Association.

Jordan, J.V., \& Hartling, L.M., (2002). New developments in Relational-Cultural Theory. In M.Ballou \& L.S. Brown (Eds.), Rethinking Mental Health and Disorders: Feminist Perspectives (pp. 48-70). New York: Guilford Publications.

Jordan, J. V., Kaplan, A. G., Miller, J. B., Stiver, I. P., Surrey, J. L. (1991). Women's growth in connection: Writings from the Stone Center. New York, NY: Guilford Press.

Julkunen, J., Gustavsson-Lilius, M., \& Hietanen, P.(2009). Anger expression, partner support, and quality of life in cancer patients. Journal of Psychosomatic Research;66(3):235-44. doi: 10.1016/j.jpsychores.2008.09.011. 
Juraskova, I., Butow, P., Robertson, R., Sharpe, L., McLeod, C., \& Hacker, N. (2003).

Post-treatment sexual adjustment following cervical and endometrial cancer: a qualitative insight. Psycho-Oncology, 12(3), 267-279. doi: 10.1002/pon.639.

Kagawa-Singer, M., Padilla, G.V., \& Ashing-Giwa, K. (2010). Health-related quality of life and culture. Seminars in Oncology Nursing, 26(1), 59-67. doi: 10.1016/j.soncn.2009.11.008.

Kamp Dush, C. M. \& Taylor, M. G. (2012). Trajectories of marital conflict across the life course: Predictors and interactions with marital happiness trajectories. Journal of Family Issues, 33, 341-368. doi: 10.1177/0192513X11409684.

Karney, B. R., Kreitz, M. A., \& Sweeney, K. E. (2004). Obstacles to ethnic diversity in marital research: On the failure of good intentions. Journal of Social and Personal Relationships, 21(4), 509-526. doi: 10.1177/0265407504044845.

Kaptein, A. A., Scharloo, M., Helder, D.I., Snoei, L., van Kempen, G.M.J., Weinman, J., van Houwelingen, J.C., \& Roos, R.A.C. (2007). Quality of life in couples living with Huntington's disease: The role of patients' and partners' illness perceptions. Quality of Life Research: An International Journal of Quality of Life Aspects of Treatment, Care \& Rehabilitation, 16(5), 793-801.

Kashy, D. A., \& Donnellan, M.B. (2012). Conceptual and methodological issues in the analysis of data from dyads and groups. In K. D. M. Snyder (Ed.), The Oxford Handbook of Personality and Social Psychology (pp. 209-238). New York, NY: Oxford University Press. doi:10.1093/oxfordhb/9780195398991.013.0009. 
Kashy, D. A., \& Snyder, D.K. . (1995). Measurement and data analytic issues in couples research. Psychological Assessment, 7(3), 338-348. http://dx.doi.org/10.1037/10403590.7.3.338.

Kayser, K. (1999). The development and testing of a psychosocial program for breast cancer patients and their partners. Paper presented at the 61st Annual Conference of the National Council on Family Relations, Irvine, CA.

Kayser, K. (2005). Enhancing dyadic coping during a time of crisis: A theory-based intervention with breast cacner patients and their partners. In T. A. Revenson, K. Kayser \& G. Bodenmann (Eds.), Couples Coping with Stress (pp. 175-194). Washington, DC: American Psychological Association.

Kayser, K. (2011). Couple Therapy. In J. R. Brandell (Ed.), Theory \& Practice in Clinical Social Work (pp. 259-288). Thousand Oaks, CA: SAGE.

Kayser, K., \& Acquati, C. (2011). Moderating factors in the relational outcomes of breast cancer patients in the Partners in Coping Program. Paper presented at the International Psycho-Oncology Society (IPOS) 13th World Congress, 18-20 October, Antalya, Turkey.

Kayser, K., Acquati, C. \& Saita, E. (2011). Coping e adattamento famigliare: Il ruolo dei fattori culturali (Coping and family adjustment: The role of cultural factors). In E. Saita (ed.), Pensare alla salute e alla malattia: legami tra mente, corpo e contest di appartenenza. Percorsi di ricerca e di intervento. (pp. 117-134)(Thinking of Health and Illness: Connections between Mind, Body and Cultural Context. Research and Intervention Pathways). Milano: Educatt. 
Kayser, K., Acquati, C., Saita, E., \& Costa, G. (2011). Coping diadico e relazione di caregiving nella sfida alla malattia oncologica. Pensare alla Salute e alla Malattia. Legami tra Mente, Corpo e Contesto di appartenza (pp. 89-116). Milano: EDUCatt.

Kayser, K., Cheung, P.K., Rao, N., Chan, Y.C., Chan, Y., \& Lo, P.H. (2014). The influence of culture on couples coping with breast cancer: a comparative analysis of couples from China, India, and the United States. Journal of Psychosocial Oncology, 32(3), 264-288. doi: 10.1080/07347332.2014.897292.

Kayser, K., Feldman, B. N., Borstelmann, N. A., \& Daniels, A. A. (2010). Effects of a randomized couple-based intervention on quality of life of breast cancer patients and their partners. Social Work Research, 34(1), 20-32. doi: 10.1093/swr/34.1.20.

Kayser, K., Saita, E., \& Acquati, C. (2011). Famiglie, fonti di stress e capacita' di fronteggiare gli eventi. Il caso della famiglia Americana (Families, stress and coping. The case of the american family). In E. Saita (Ed.), Pensare alla salute $e$ alla malattia: legami tra mente, corpo e contest di appartenenza. Percorsi di ricerca e di intervento (pp. 69-88) (Thinking of Health and Illness: Connections between Mind, Body and Cultural Context. Research and Intervention Pathways). Milano: Educatt.

Kayser, K., \& Scott, J.L. (2008). Helping Couples Cope with Women's Cancers. An Evidence-Based Approach for Practitioners. New York, NY: Springer.

Kayser, K., \& Sormanti, M. (2002). A follow-up study of women with cancer: Their psychological well-being and close relationships. Social Work in Health Care, 35(1/2), 391-406. 
Kayser, K., Sormanti, M., \& Strainchamps, E. (1999). Women coping with cancer: The influence of relationship factors on psychosocial adjustment. Psychology of Women Quarterly, 23(4), 725-739. doi:10.1111/j.1471-6402.1999.tb00394.x.

Kayser, K., Watson, E., \& Andrade, J. T. (2007). Cancer as a we-disease: Examining the process of coping from a relational perspective. Families Systems and Health, 25(4), 404- 418. doi: 10.1037/1091-7527.25.4.404.

Kenny, D.A. (1996). Models of nonindependence in dyadic research. Journal of Social and Personal Relationships, 13(2), 279-294. doi: 10.1177/0265407596132007.

Kenny, D.A. (2013). Actor-Partner Interdependence Model. Available at http://davidakenny.net/webinars/powerpoints/Dyad/Standard/APIM.ppt.

Kenny, D. A., \& Judd, C. A. (1986). Consequences of violating the independence assumption in analysis of variance. Psychological Bulletin, 99(3), 422-431. doi: 10.1037/0033-2909.99.3.422.

Kenny, D. A., Kashy, D. A., \& Bolger, N. (1998). Data analysis in Social Psychology. In S. F. D. Gilbert, \& G. Lindzey (Eds.), Handbook of social psychology (4th Edition ed., pp. 233-265). Boston: McGraw-Hill.

Kenny, D. A., Kashy, D.A., \& Cook, W.L. (2006). Dyadic Data Analysis. New York, NY: The Guilford Press

Kline, R.B. (2011). Principles and practice of structural equation modeling, 3ed. New York, NY: The Guilford Press

Kiecolt-Glaser , J.K., Bane, C., Glaser, R., \& Malarkey, W.B. (2003). Love, marriage, and divorce: newlyweds' stress hormones foreshadow relationship changes. Journal 
of Consulting and Clinical Psychology, 71(1), 176-88. doi: 10.1037/0022006x.71.1.176.

Kiecolt-Glaser, J. K., \& Glaser, R. (2002). Depression and immune function: Central pathways to morbidity and mortality. Journal of Psychosomatic Research, 53(4), 873-876. doi: http://dx.doi.org/10.1016/s0022-3999(02)00309-4.

Kiecolt-Glaser, J. K., McGuire, L., Robles, T., \& Glaser, R. (2002).

Psychoneuroimmunology: Psychological influences on immune function and health. Journal of Consulting and Clinical Psychology, 70(3), 537-547. doi: 10.1037//0022-006x.70.3.537

Kiecolt-Glaser, J. K., \& Newton, T. (2001). Marriage and health: His and Hers. Psychological Bullettin, 127(4), 472-503. http://dx.doi.org/10.1037/00332909.127.4.472.

Kienle, R., Luszczynska, A. Pfüller, B., \& Knoll, N. (2009). Appraisal detection bias and well-being in close relationships: couples experiencing assisted reproduction treatment. Applied Psychology Health and Well-Being, l(2):165 - 187. doi:10.1111/j.1758-0854.2009.01011.x.

Kim, Y., Carver, C.S., Shaffer , K.M., Gansler, T., \& Cannady, R.S. (2015). Cancer caregiving predicts physical impairments: Roles of earlier caregiving stress and being a spousal caregiver. Cancer, 121(2), 302-310. doi: 10.1002/cncr.29040.

Kim, Y., Kashy, D.A., Wellisch, D.K., Spillers, R. L., Kaw, C.K., \& Smith, T.G. (2008). Quality of life of couples dealing with cancer: Dyadic and individual adjustment among breast and prostate cancer survivors and their spousal caregivers. Annals of Behavioral Medicine, 35(2), 230-238. doi: 10.1007/s12160-008-9026-y. 
Kim, Y., Schulz, R., \& Carver, C.S. (2007). Benefit-finding in the cancer caregiving experience. Psychosomatic Medicine, 69(3), 283-291. doi:

\subsection{7/PSY.0b013e3180417cf4}

Kim, Y. Spillers, R.L., \& Hall, D.L. (2012). Quality of life of family caregivers 5 years after a relative's cancer diagnosis: Follow-up of the national quality of life survey for caregivers. Psycho-Oncology, 21(3), 273-281. doi: 10.1002/pon.1888.

Kim, Y., van Ryn, M., Jensen, R.E., Griffin, J.M., Potosky, A., \& Rowland, J. (2015). Effects of gender and depressive symptoms on quality of life among colorectal and lung cancer patients and their family caregivers. Psycho-Oncology, 24(1), 95-105. doi: 10.1002/pon.3580.

King, M.T., Kenny, P., Shiell, A., Hall, J., \& Boyages, J. (2000). Quality of life three months and one year after first treatment for early stage breast cancer: Influence of treatment and patient characteristics. Quality of Life Research, 9(7), 789-800.

Kinsinger, S.W., Laurenceau, J.P., Carver, C.S., \& Antoni, M.H. (2011). Perceived partner support and psychosexual adjustment to breast cancer. Psychology \& Health, 26(12), 1571-1588 doi: 10.1080/08870446.2010.533771.

Kitrungrote, L., \& Cohen, M. Z. (2006). Quality of life of family caregivers of patients with cancer: A literature review. Oncology Nursing Forum, 33(3), 625-632.

Koch, B., Hauschild, S., \& Schmidt-Denter, U. (2001). Dynam-ische Abhängigkeiten in der Paarbeziehung beim $U$ “'bergang zur Elternschaft [Dynamic dependencies in couple's relation-ships during the transmission to parenthood]. Poster presentation at the 2nd Munic Symposium of Family Psychology. 
Koch, L., Jansen, L., Herrmann, A., Stegmaier, C., Holleczek, B., Singer, S., Brenner, H., \& Arndt, V. (2013) Quality of life in long-term breast cancer survivors - A 10-year longitudinal population-based study. Acta Oncologica, 52(6), 1119-1128. doi: $10.3109 / 0284186 X .2013 .774461$

Koh, H.K. (2009). Towards the elimination of cancer disparities. Medical and health perspectives. New York, NY: Springer.

Kohut, H. (1978). The Search for the Self. Madison, CN: International Universities Press. Kraemer, L.M., Stanton, A.L., Meyerowitz, B.E., Rowland, J.H., \& Ganz, P.A. (2011). A longitudinal examination of couples coping strategies as predictors of adjustment to breast cancer. Journal of Family Psychology, 25(6), 963-972. doi: $10.1037 / \mathrm{a} 0025551$.

Kramer, U., Ceschi, G., Van der Linden, M., \& Bodenmann, G. (2005). Individual and dyadic coping strategies in the aftermath of a traumatic experience. Swiss Journal of Psychology, 64, 241-248. http://dx.doi.org/10.1024/1421-0185.64.4.241.

Kroenke, C.H., Rosner, B., Chen, W.Y., Kawachi, I., Colditz, G.A., \& Holmes, M.D. (2004). Functional impact of breast cancer by age at diagnosis. Journal of Clinical Oncology, 22(10), 1849-1856. doi: 10.1200/JCO.2004.04.173.

Kruger, K. (2005). Relationship and relational mutuality as predictors of well-being and six constructs of well-being. ETD Collection for Tennessee State University. Paper AAI3187596.Available at:

http://digitalscholarship.tnstate.edu/dissertations/AAI3187596.

Krüger, A., Leibbrand, B., Barth, J., Berger, D., Lehmann, C., Koch, U., \& Mehnert, A. (2009). Course of psychosocial distress and health-related quality of life in patients 
at different age groups during cancer rehabilitation. Zeitschrift fur

Psychosomatische Medizin und Psychotherapie, 55(2), 141-161.

Kuenzler, A., Hodgkinson, K., Zindel, A., Bargetzi, M., \& Znoj, H.J. (2011). Who cares, who bears, who benefits? Female spouses vicariously carry the burden after cancer diagnosis. Psychology \& Health, 26(3), 337-352. doi:10.1080/08870440903418877.

Kuijer, R. G., Buunk, B.P., De Jong, G.M., Ybema, J.F., \& Sanderman, R. (2004).

Effects of a brief intervention program for patients with cancer and their partners on feelings of inequality, relationship quality, and psychological distress. PsychoOncology, 13(5), 321-334. doi: 10.1002/pon.749.

Kuijer, R. G., Ybema, J., F., Buunk, B. P., DeJong, G. M., Thijs-Boer, F., \& Sanderman, R. (2000). Active engagement, protective buffering, and overprotection: Three ways of giving support by intimate partners of patients with cancer. Journal of Social and Clinical Psychology, 19(2), 256-275. doi: 10.1521/jscp.2000.19.2.256.

Künzler, A., Nussbeck, F.W., Moser, M.T., Bodenmann, G., \& Kayser, K. (2014). Individual and dyadic development of personal growth in couples coping with cancer. Supportive Care Cancer, 22(1):53-62. doi:10.1007/s00520-013-1949-6.

Kunzler, A., Zindel, A., Bargetzi, M., \& Znoji, H. J. (2005). Couples coping with a recent diagnosis of cancer. Annual Conference of the European Health Psychology Society. Galway, Ireland.

Kuyper, M. B., \& Wester, F. (1998). In the shadow: The impact of chronic illness on the patients's partner. Qualitative Health Research, 8(2), 237-253. doi: $10.1177 / 104973239800800207$. 
Labouvie-Vief G. (2003). Dynamic integration: Affect, cognition, and the self in adulthood. Current Directions in Psychological Science, 12(6), 201-206. doi: 10.1046/j.0963-7214.2003.01262.x

Labouvie-Vief, G., Hakim-Larson, J., \& Hobart, C. (1987). Age, ego level, and the lifespan development of coping and defense process. Psychology and Aging, 2(3), 286293. http://dx.doi.org/10.1037/0882-7974.2.3.286.

Lafaye, A., Petit, S., Richaud, P., Houede, N., Baguet, F., \& Cousson-Gelie, F. (2014). Dyadic effects of coping strategies on emotional state and quality of life in prostate cancer patients and their spouses. Psycho-Oncology, 23(7), 797-803. doi:10.1002/pon.3483.

Landis, M., Bodenmann, G., Bradbury, T.N., Brandstätter, V., Peter-Wight, M., Backes, S., Sutter-Stickel, D., Nussbeck, F.W. (2014). Commitment and dyadic coping in long-term relationships. GeroPsych: The Journal of Gerontopsychology and Geriatric Psychiatry, 27(4), 139-149. doi: 10.1024/1662-9647/a000112.

Landis, M., Peter-Wight, M., Martin, M., \& Bodenmann, G. (2013). Dyadic coping and marital satisfaction of older spouses in long-term marriage. GeroPsych: The Journal of Gerontopsychology and Geriatric Psychiatry, 26(1), 39-47. http://dx.doi.org/10.1024/1662-9647/a000077.

Landwehr, M.S., Watson, S.E., Macpherson, C.F., Novak, K.A., \& Johnson, R.H. (2016). The cost of cancer: A retrospective analysis of the financial impact of cancer on young adults. Cancer Medicine, Early View. Article first published online 7 February, 2016. doi: 10.1002/cam4.657. 
Langer, S. L., Brown, J. D., \& Syrjala, K. L. (2009). Intrapersonal and interpersonal consequences of protective buffering among cancer patients and caregivers. Cancer, 115(18 suppl), 4311-4325. doi: 10.1002/cncr.24586.

Laszloffy, T. (2002). Rethinking family development theory: Teaching with the Systemic Family Development (SFD) model. Family Relations, 51, 206-214. doi:

10.1111/j.1741-3729.2002.206098.x.

LaVeist, T.A. (2005). Disentangling race and socioeconomic status: A key to solving health disparities. Journal of Urban Health, 82(2 Suppl), 26-34.

Lauer, R. H. \& Lauer, J. C. (2009). Marriage and family: The quest for intimacy. New York, NY: McGraw-Hill.

Lauer, R. H., Lauer, J. C., \& Kerr, S. T. (1990). The long-term marriage: Perceptions of stability and satisfaction. International Journal of Aging and Human Development, 31(3), 189-195. doi: 10.2190/H4X7-9DVX-W2N1-D3BF .

Lavery, J. F., \& Clarke, V. A. (1999). Prostate cancer: Patients' and spouses' coping and marital adjustment. Psychology, Health \& Medicine, 4(3), 279-302. doi:10.1080/135485099106225.

Lavner, J. A., \& Bradbury, T. N. (2010). Patterns of change in newlyweds' marital satisfaction. Journal of Marriage and Family, 72(5), 1171-1187. doi: 10.1111/j.1741-3737.2010.00757.x.

Lavner, J. A., Bradbury, T. N., \& Karney, B. R. (2012). Incremental change or initial differences? Testing two models of marital deterioration. Journal of Family Psychology, 26(4), 606-616. doi: 10.1037/a0029052. 
Lavner, J. A., Karney, B. R., \& Bradbury, T. N. (2013). Newlyweds' optimistic forecasts of their marriage: For better or for worse? Journal of Family Psychology, 27(4), 531-540. doi:10.1037/a0033423.

Lawson, A.K., Klock, S.C., Pavone, M.E., Hirshfeld-Cytron, J., Smith, K.N., Kazer, R.R. (2014). Prospective study of depression and anxiety in female fertility preservation and infertility patients. Fertility \& Sterility, 102(5), 1377-1384. doi:

10.1016/j.fertnstert.2014.07.765.

Lazarus, R. S. (1966). Psychological stress and the coping process. New York: McGrawHill.

Lazarus, R. S. (1990). Stress, coping, and illness. In H. S. Friedman (Ed.), Personality and disease (pp. 97-120). Oxford, England: John Wiley \& Sons.

Lazarus, R. S. (1990). Theory-based stress measurement. Psychological Inquiry, 1(1), 313.

Lazarus, R. S. (1993). Why we should think of stress as a subset of emotion. In L. Goldberger \& S. Breznitz (Eds.), Handbook of stress: Theoretical and clinical aspects (2nd ed., pp. 21-39). New York, NY: Free Press.

Lazarus, R. S., \& Folkman, S. (1984). Stress, appraisal, and coping. New York, NY: Springer

Lazarus, R. S. \& Folkman., S. .(1987). Transactional theory and research on emotions and coping. European Journal of Personality, 1(3), 141-169. Doi: 10.1002/per.2410010304. 
Ledermann, T., Bodenmann, G., \& Cina, A. (2007). The efficacy of the Couples Coping Enhancement Training (CCET) in improving relationship quality. Journal of Social and Clinical Psychology, 26(8), 940-959. doi: 10.1521/jscp.2007.26.8.940.

Ledermann, T., Bodenmann, G., Gagliardi, S., Charvoz, L., Rossier, J., Bertoni, A., \& Iafrate, R. (2010). Psychometrics of the Dyadic Coping Inventory (DCI) in three language groups. Swiss Journal of Psychology, 69(4):201-212. doi: 10.1024/14210185/a000024.

Ledermann, T., \& Kenny, D.A. (2012). The Common Fate Model for dyadic data: Variations of a theoretically important but underutilized model. Journal of Family Psychology, 26(1), 140-148. doi: 10.1037/a0026624.

Ledermann, T. \& Macho, S. (2014). Analyzing change at the dyadic level: The common fate growth model. Journal of Family Psychology, 28(2), 204-213.. http://dx.doi.org/10.1037/a0036051.

Ledermann, T., Macho, S., \& Kenny, D. A. (2011). Assessing mediation in dyadic data using the actor-partner interdependence model. Structural Equation Modeling, 18, 595-612. doi: 10.1080/10705511.2011.607099.

Lee, Y.H., Liao, Y.C.., Liao, W.Y., Shun, S.C., Liu, Y.C., Chan, J.C., Yu, C.Y., Yang, P.C., Lai, Y. H. (2013). Anxiety, depression and related factors in family caregivers of newly diagnosed lung cancer patients before first treatment. Psycho-Oncology, 22(11), 2617-2623. doi: 10.1002/pon.3328

Lee, E., Menon, U., Nandy, K., Szalacha, L., Kviz, F., Cho, Y., Miller, A., \& Park, H. (2014). The effect of a couples intervention to increase breast cancer screening 
among Korean Americans. Oncology Nursing Forum, 41(3), E185-93. doi:

10.1188/14.ONF.E185-E193.

Lee, H.B., \& Wonshik, H. (2014). Unique features of young age breast cancer and its management. Journal of Breast Cancer, 17(4), 301-307. doi:

10.4048/jbc.2014.17.4.301.

Lee, R., Wakefield, A., Foy, S., Howell, S.J., Wardley, A.M., \& Armstrong, A.C. (2011). Facilitating reproductive choices: The impact of health servoces on the experiences of young women with breast cancer. Psycho-Oncology, 20(10), 1044-1052. doi: 10.1002/pon.1826.

Lepore, S.J. \& Revenson, T.A. (2007). Social constraints on disclosure and adjustment to cancer. Social and Personality Psychology Compass, 1(1), 313-333.

Leung, J., Pachana, N.A., \& McLaughlin, D. (2014). Social support and health-related quality of life in women with breast cancer: a longitudinal study. Psycho-Oncology, 23(9), 1014-1020. doi: 10.1002/pon.3523.

Levenson, R., Carstensen, L., \& Gottman, J. (1994) The influence of age and gender on affect, physiology and their interrelations: A study of long-term marriages. Journal of Personality and Social Psychology, 67(1), 56-68. http://dx.doi.org/10.1037/00223514.67.1.56,

Levesque, C., Lafontaine, M.F., Caron, A., \& Fitzpatrick, J. (2014a). Validation of the english version of the Dyadic Coping Inventory. Measurement and Evaluation in Counseling and Development, 47(3), 215-225. doi: 10.1177/0748175614522272. 
Levesque, C., Lafointaine, M., Caron, A., Flesch, J.L., \& Bjornson. (2014b). Dyadic empathy, dyadic coping, and relationship satisfaction: A dyadic model. Europe's Journal of Psychology, 10(1), 118-134. doi: 10.5964/ejop.v10i.697.

Levin, A. O., Carpenter, K.M., Fowler, J.M., Brothers, B.M., Andersen,B.L., \& Maxwell, G.L. (2010). Sexual morbidity associated with poorer psychological adjustment among gynecological cancer survivors. International Journal of Gynecological Cancer, 20(3), 461-470. doi: 10.1111/IGC.0b013e3181d24ce0.

Lewis, F. M. (2010). The Family's "Stuck Points" in Adjusting to Cancer. In J. C. Holland, Breitbart, W.S., Jacobsen, P.B., Lederberg, M.S., Loscalzo, M.J., \& McCorkle, R. (Ed.), Psycho-Oncology (pp. 511-515). New York, NY: Oxford University Press.

Lewis, F.M., Cochrane, B.B., Fletcher, K.A., Zahlis, E.H., Shands, M.E., Gralow, J.R., Wu, S.M., \& Schmitz, K. (2008). Helping Her Heal: A pilot study of an educational counseling intervention for spouses of women with breast cancer. PsychoOncology, 17(2), 131-137. doi: 10.1002/pon.1203.

Lewis, F. M., \& Deal, L. W. (1995). Balancing our lives: A study of the married couple's experience with breast cancer recurrence. Oncology Nursing Forum, 22(6), 943953.

Lewis, F. M., Fletcher, K.A., Cochrane, B.B., \& Fann JR. (2008). Predictors of depressed mood in spouses of women with breast cancer. Journal of Clinical Oncology, 26(8), 1289-1295. doi: 10.1200/JCO.2007.12.7159. 
Lewis, F. M., Woods, N.F., Hough, E.E., \& Bensley, L.S. (1989). The family's functioning with chronic illness in the mother: the spouse's perspective. Social Science \& Medicine, 29(11), 1261-1269. doi: 10.1016/0277-9536(89)90066-x.

Liang, B., Tracy, A., Taylor, C., Williams, L., Jordan, J., \& Miller, J. B. (2002). The Relational Health Indices: A study of women's relationships. Psychology of Women's Quarterly, 26(1), 25-35. doi:10.1111/1471-6402.00040.

Lichtman, R. R., Taylor, S.E., Wood, J.V. (1988). Social support and marital adjustment after breast cancer. Journal of Psychosocial Oncology, 5(3), 47-74. Doi: 10.1300/J077v05n03_03.

Lim, J.W., Gonzalez, P., Wang-Letzkus, M.F., \& Ashing-Giwa, K.T. (2009). Understanding the cultural health belief model influencing health behaviors and health-related quality of life between Latina and Asian-American breast cancer survivors. Supportive Care in Cancer, 17(9):1137-47. doi: 10.1007/s00520-008$0547-5$.

Lin, I., Fee, H. R., \& Wu, H. (2012). Negative and positive caregiving experiences: A closer look at the intersection of gender and relationship. Family Relations, 61(2), 343-358. doi:10.1111/j.1741-3729.2011.00692.x.

Lin, H. C., Lin, W.C., Lee, T.Y., \& Lin, H.R. (2013). Living experiences of male spouses of patients with metastatic cancer in Taiwan. Asian Pacific Journal of Cancer Prevention, 14(1), 255-259. doi: 10.7314/apjcp.2013.14.1.255.

Lindau, S. T., Surawska, H., Paice, J., \& Baron, S. R. (2011). Communication about sexuality and intimacy in couples affected by lung cancer and their clinical-care providers. Psycho-Oncology, 20(2), 179-185. doi:10.1002/pon.1787 
Litzelman, K., \& Yabroff, K.R. (2015). How are spousal depressed mood, distress, and quality of life associated with risk of depressed mood in cancer survivors? Longitudinal findings from a national sample. Cancer Epidemiology, Biomarkers \& Prevention, 24; 969. doi:10.1158/1055-9965.EPI-14-1420.

Llewellyn, C.D., Horney, D.J., McGurk, M., Weinman, J., Herold, J., Altman, K., \& Smith, H.E. (2013). Assessing the psychological predictors of benefit finding in patients with head and neck cancer. Psycho-Oncology, 22(1), 97-105. doi: 10.1002/pon.2065.

Loaring, J. M., Larkin, M., Shaw, R., \& Flowers, P. (2015). Renegotiating sexual intimacy in the context of altered embodiment: The experiences of women with breast cancer and their male partners following mastectomy and reconstruction. Health Psychology, 34(4), 426-436. doi:10.1037/hea0000195.

Luong, G., Charles, S. T., \& Fingerman, K. L. (2011). Better with age: Social relationships across adulthood. Journal of Social and Personal Relationships, 28(1), 9-23. doi:10.1177/0265407510391362.

Luszczynska, A., Boehmer, S., Knoll, N., Schulz, U., \& Schwarzer, R. (2007). Emotional support for men and women with cancer: Do patients receive what their partners provide? International Journal of Behavioral Medicine, 14(3),156-163.

Luutonen, S., Vahlberg, T., Eloranta, S., Hyvari, H., \& Salminen, E. (2011) Breast cancer patients receiving postoperative radiotherapy: Distress, depressive, symptoms and unmet needs of psychosocial support. Radiotherapy Oncology, 100(2), 299-303. doi: 10.1016/j.radonc.2011.01.014. 
Lyon, B. L. (2012). Stress, Coping and Health: A conceptual overview. In V. Hill Rice (Ed.), Handbook of Stress, Coping, and Health. Implications for Nursing Research, Theory, and Practice (2nd Edition ed., pp. 2-20). Thousand Oaks; CA: Sage.

Lyons, K.S., Lee, C.S., Bennett, J.A., Nail, L.M., Fromme, E., Hiatt, S.O., \&Sayer, A. G. (2014). Symptom Incongruence Trajectories in Lung Cancer Dyads. Journal of Pain and Symptom Management, 48(6), 1031-1040. http://dx.doi.org/10.1016/j.jpainsymman.2014.02.004

Lyons, R. F., Mickelson, K. D., Sullivan, M. J. L., \& Coyne, J. C. (1998). Coping as a communal process. Journal of Social and Personal Relationships, 15(5), 579-605. doi: $10.1177 / 0265407598155001$.

Lyons, K.S., Stewart, B.J., Archbold, P.G., \& Carter, J.H. (2009). Optimism, pessimism, mutuality, and gender: predicting 10-year role strain in Parkinson's disease spouses. The Gerontologist, 49(3), 378-387. doi: 10.1093/geront/gnp046.

Lyons, R. F., \& Sullivan, M. J. L. (1998). Curbing loss in illness and disability: A relationship perspective. In J. H. Harvey (Ed.), Perspectives on Loss: A sourcebook (pp. 137-152). Philadelphia: Brunner/Mazel.

MacKinnon, D.P. (2008). An introduction to statistical mediation analysis. New York, NY: Routledge.

MacKinnon, D.P., Lockwood, C.M., \& Williams, J. (2004). Confidence limits for the indirect effect: Distribution of the product and resampling methods. Multivariate Behavioral Research, 39(1), 41-62. doi: 10.1207/s15327906mbr3901_4 
Magai, C. (2008). Attachment in middle and later life. In J. Cassidy, P.R. Shaver (Eds.), Handbook of attachment: Theory, research and clinical applications, 2nd edition. New York, NY: Guildford Press.

Magsamen-Conrad, K, Checton, M. G., Venetis, M. K., \& Greene, K. (2015). Communication efficacy and couples' cancer management: Applying a dyadic appraisal model. Communication Monographs. doi:

10.1080/03637751.2014.971415.

Manganiello, A., Hoga, L.A., Reberte, L.M., Miranda, C.M., \& Rocha, C.A. (2011). Sexuality and quality of life of breast cancer patients post mastectomy. European Journal of Oncology Nursing, 15(2), 167-172. doi:10.1016/j.ejon.2010.07.008.

Manne, S. \& Badr., H. (2008). Intimacy and relationship processes in couples' psychological adaptation to cancer. Cancer, 112(11Suppl), 2541-2555. doi:10.1002/cncr.23450.

Manne, S., \& Badr, H. (2010). Intimacy processes and psychological distress among couples coping with head and neck or lung cancers. Psycho-oncology, 19(9), 941954. doi: 10.1002/pon.1645.

Manne, S., Badr, H., \& Kashy, D.A. (2012). A longitudinal analysis of intimacy processes and psychological distress among couples coping with head and neck or lung cancers. Journal of Behavioral Medicine, 35(3), 334-346. doi:10.1007/s 10865011-9349-1.

Manne, S., Badr, H., Zaider, T., Nelson, C., \& Kissane, D. (2010). Cancer-related communication, relationship intimacy, and psychological distress among couples 
coping with localized prostate cancer. Journal of Cancer Survivorship, 4(1), 74-85. doi:10.1007/s11764-009-0109-y.

Manne, S.L., Kissane, D., Zaider, T., Kashy, D., Lee, D., Heckman, C., \& Virtue, S.M. (2015). Holding back, intimacy, and psychological and relationship outcomes among couples coping with prostate cancer. Journal of Family Psychology, 29(5), 708-719. http://dx.doi.org/10.1037/fam0000096.

Manne, S., Ostroff, J., Norton, T. R., Fox, K., Goldstein, L., \& Grana, G. (2006). Cancerrelated relationship communication in couples coping with early stage breast cancer. Psycho-Oncology, 15(3), 234-247. doi: 10.1002/pon.941.

Manne, S., Ostroff, J., Rini, C., Fox, K., Goldstein, L., \& Grana, G. (2004). The interpersonal process model of intimacy: The role of self-disclosure, partner disclosure, and partner responsiveness in interactions between breast cancer patients and their partners. Journal of Family Psychology, 18, 589-599. doi:10.1037/08933200.18.4.589.

Manne, S.L., Ostroff, J.S., Winkel, G., Fox, K., Grana, G., Miller, E., Ross, S., \& Frazier, T. (2005). Couple-focused group intervention for women with early stage breast cancer. Journal of Consulting and Clinical Psychology, 73(4), 634-646. http://dx.doi.org/10.1037/0022-006X.73.4.634.

Manne, S., Ostroff, J. S., Winkel, G., Goldstein, L., Fox, K., \& Grana, G. (2004). Posttraumatic growth after breast cancer: Patient, partner, and couple perspectives. Psychosomatic Medicine, 66(3), 442-454. doi: 10.1177/153476560501100406.

Manne, S., Rini, C., Rubin, S., Rosenblum, N., Bergman, C., Edelson, M., \& Rocereto, T. (2008). Long-term trajectories of psychological adaptation among women 
diagnosed with gynecological cancers. Psychosomatic Medicine, 70(6), 677-687. doi: 10.1097/PSY.0b013e31817b935d.

Manne, S.L., Rubin, S., Edelson, M., Rosenblum, N., Bergman, C., Hernandez, E., Carlson, J., Rocereto, T., Winkel, G. (2007). Coping and communication-enhancing intervention versus supportive counseling for women diagnosed with gynecological cancers. Journal of Consulting and Clinical Psychology, 75(4), 615-628. http://dx.doi.org/10.1037/0022-006X.75.4.615.

Manne, S., Sherman, M., Ross, S., Ostroff, J., Heyman, R.E., \& Fox, K. (2004). Couples' support-related communication, psychological distress, and relationship satisfaction among women with early state breast cancer. Journal of Consulting and Clinical Psychology, 72(4), 660-670. Doi: 10.1037/0022-006X.72.4.660.

Manne, S., Winkel, G., Grana, G., et al. (2005). Couple-focused group intervention for women with early stage breast cancer. Journal of Consulting and Clinical Psychology, 73(4), 634-646. http://dx.doi.org/10.1037/0022-006X.73.4.634.

Manuel, J.C., Burwell, S.R., Crawford, S.L., Lawrence, R.H., Farmer, D.F., Hege, A., Phillips, K., \& Avis, N.E. (2007). Younger women's perceptions of coping with breast cancer. Cancer Nursing, 30(2), 85-94.

Martin, M., Peter-Wight, M., Braun, M., Hornung, R., \& Scholz, U. (2009). The 3-phase model of dyadic adaptation to dementia: Why it might sometimes be better to be worse. European Journal of Ageing, 6(4), 291-301. doi: 10.1007/s10433-009-01295.

Martire, L.M., Schulz, R., Helgeson, V.S., Small, B.J., Saghafi, E.M., \& Ann, M.L.S. (2010). Review and meta-analysis of couple-oriented interventions for chronic 
illness. Annals of Behavioral Medicine, 40(3): 325-342.doi: 10.1007/s12160-0109216-2.

Martire, L.M., Stephens, M.A.P., Druley, J.A., Wojno, W.C. (2002). Negative reactions to received spousal care: Predictors and consequences of miscarried support. Health Psychology, 21, 167-176. doi: 10.1037/0278-6133.21.2.167

Mattessich, P., \& Hill, R. (1987). Life cycle and family development. In M. B. Sussman and S. K. Steinmetz (eds.). Handbook of Marriage and the Family,. New York, NY: Plenum press.

Matthews, B.A., Baker, F., \& Spillers, R.L. (2004). Oncology professionals and patient requests for cancer support services. Supportive Care in Cancer, 12(10), 731738.doi: 10.1007/s00520-004-0647-9.

Maughan, K., Heyman, B., \& Matthews, M. (2002). In the shadow of risk. How men cope with a partner's gynaecological cancer. International Journal of Nursing Studies, 39(1), 27-34.

Mazanec, S.R., Daly, B.J., Douglas, S.L., \& Lipson, A.R. (2011). Work productivity and health of informal caregivers of persons with advanced cancer. Research in Nursing and Health, 34(6), 483- 495. http://dx.doi.org/10.1002/nur.20461.

McClure, K. S., Nezu, A. M., Nezu, C. M., O’Hea, E. L.,\& McMahon, C. (2012). Social problem solving and depression in couples coping with cancer. Psycho-Oncology, 21 (1), 11-19. doi:10.1002/pon.1856.

McCrae, R.R. (1989). Age differences and changes in in the use of coping mechanisms. Journal of Gerontology: Psychological Sciences, 44(6), 161-169. 
McLean, L. M., \& Jones, J.M. (2007). A review of distress and its management in couples facing end-of-life cancer. Psycho-Oncology, 16(7), 603-616. doi: 10.1002/pon.1196.

Meegan S., \& Berg, C.A. (2002). Contexts, functions, forms, and processes of collaborative everyday problem solving in older adulthood. International Journal of Behavioral Development, 26(1), 6-15. doi: 10.1080/01650250143000283.

Mehnert, A. \& Koch, U. (2008). Psychological comorbidity and health-related quality of life and its association with awareness, utilization, and need for psychosocial support in a cancer register-based sample of long-term breast cancer survivors. Journal of Psychosomatic Research, 64(4), 383-391. doi:

10.1016/j.jpsychores.2007.12.005.

Meier, C., Bodenmann. G., Mörgeli, H., \& Jenewein, J.. (2011). Dyadic coping, quality of life, and psychological distress among chronic obstructive pulmonary disease patients and their partners. International Journal of Chronic Obstructive Pulmonary Disease. 6, 583-596. doi http://dx.doi.org/10.2147/copd.s24508.

Meier, C., Bodenmann, G., Moergeli, H, Peter-Wight, H. Martin, M., Buchi, S., Jenewein, J. (2012). Dyadic coping among couples with COPD: A pilot study. Journal of Clinical Psychology in Medical Settings, 19(3), 243-254. doi 10.1007/s 10880-011-9279-7.

Mellon, S., Northouse, L.L., \& Weiss, L.K. (2006). A population based study of the quality of life of cancer survivors and their family caregivers. Cancer Nursing, 29(2), 120-131. 
Meneses, K., McNees, P., Azuero, A., \& Jukkala, A. (2010). Development of the Fertility and Cancer Project: An Internet Approach to Help Young Cancer Survivors. Oncology Nursing Forum, 37(2), 191-197.doi: 10.1188/10.ONF.191-197.

Mertz, B.G., Bistrup, P.E., Johansen, C., Dalton, S.O., Deltour, I., Kehlet, H., \& Kroman, N. (2012). Psychological distress among women with newly diagnosed breast cancer. European Journal of Oncology Nursing, 16(4):439-443. doi: 10.1016/j.ejon.2011.10.001.

Merz, E. L., Malcarne, V. L., Ko, C. M., Sadler, M., Kwack, L., Varni, J. W., \& Sadler, G. R. (2011).Dyadic concordance among prostate cancer patients and their partners and health related quality of life:Does it matter?. Psychological Health, 26(6), 651666. doi: 10.1080/08870441003721251.

Meuwly, N., Bodenmann, G., Germann, J., Bradbury, T. N., Ditzen, B., \& Heinrichs, M. (2012). Dyadic coping, insecure attachment, and cortisol stress recovery following experimentally-induced stress. Journal of Family Psychology, 6, 937-947. doi: 10.1037/a0030356.

Meyers, L.S., Gamst, G., \& Guarino, A.J. (2006). Applied Multivariate Analysis. Design and Interpretation.Thousand Oaks, CA:Sage Publications.

Miedema, B., Easley, J.K., \& Robinson, L.M. (2013). Comparing urban and rural young adult cancer survivors' experiences: a qualitative study. Rural and Remote Health, 13(2):2324. Available online at http://www.rrh.org.au.

Milbury K, \& Badr H. (2013). Sexual problems, communication patterns, and depressive symptoms in couples coping with metastatic breast cancer. Psycho-Oncology, 22(4):814-822. doi: 10.1002/pon.3079. 
Milbury, K., Badr, H., \& Carmack, C.L. (2012). The role of blame in the psychosocial adjustment of couples coping with lung cancer. Annals of Behavioral Medicine, 44(3), 331-340. doi: 10.1007/s12160-012-9402-5.

Miller, J. B. (1976). Toward a new psychology of women. Boston: Beacon Press.

Miller, J. B. (1984). The development of women's sense of self. Work in Progress No. 12. Wellesley, MA: Stone Center Working Papers Series.

Miller, J. B. (1989). Connections, disconnections and violations Work in Progress, No33, Wellesley, MA: Stone Center Working Paper Series.

Miller, J. B., \& Stiver, 1. (1997). The healing connection: How women form relationships in therapy and life. Boston: Beacon Press.

Moreira, H., \& Canavarro, M.C. (2013). Psychosocial adjustment and marital intimacy among partners of patients with breast cancer: A comparison study with partners of healthy women. Journal of Psychosocial Oncology, 31(3), 282-304. doi:

10.1080/07347332.2013.778934.

Morrow, P.K., Broxson, A.C., Munsell, M.F., Basen-Enquist, Rosebblum, C.K., Schover, L. R., Nguyen, L.H., Hsu, L., Castillo, L., Hahm, K.M., Litton, J.K., Kwiatkowski, D.N., \& Hortobagyi, G.N. (2014). Effect of age and race on quality of life in young breast cancer survivors. Clinical Breast Cancer, 14(2), e21-e31. doi: http://dx.doi.org/10.1016/j.clbc.2013.10.003.

Murray, S.L., Holmes, J.G., Bellavia, G., Griffin, D.W., \& Dolderman, D. (2002). Kindred spirits? The benefits of egocentrism in close relationships. Journal of Personality and Social Psychology, 82(4):563-581. doi.org/10.1037/00223514.82.4.563. 
Nakaya, N., Saito-Nakaya, K., Bidstrup, P. E., Dalton, S. O., Frederiksen, K., StedingJessen, M., \&.Johansen, C. (2010). Increased risk of severe depression in male partners of women with breast cancer. Cancer, 116(23), 5527-5534. doi:10.1002/cncr.25534.

National Institute of Aging (2015). Health and Aging: Menopause. Available at https://www.nia.nih.gov/health/publication/menopause

Naumann, S. (2004). Partnerschaft und Gesundheit. Die Bedeu-tung von Konfliktgespra“chen und subjektiver Beziehungsqu-alita“t fu“r die Gesundheit der Partner. [Close relationshipsand health: The significance of conflict discussions and subjective relationship quality for partners' health]. Unpublished dissertation. Braunschweig: Technische Universitat Braunschweig.

Neff, L. A., \& Broady, E. F. (2011). Stress resilience in early marriage: Can practice make perfect? Journal of Personality and Social Psychology, 101(5), 10501067.doi:10.1037/a0023809.

Neuling, S. J., \& Winefield, H.R. (1988). Social support and recovery after surgery for breast cancer: frequency and correlates of supportive behaviors by family friends and surgeon. Social Science \& Medicine, 27(4), 385-392. doi: 10.1016/02779536(88)90273-0.

Northouse, L.L. (1989). A longitudinal study of the adjustment of patients and husbands to breast cancer. Oncology Nursing Forum, 16(4), 511-516.

Northouse L.L., Dorris. G., \& Charron-Moore C. (1995). Factors affecting couples’ adjustment to recurrent breast cancer. Social Science \& Medicine, 41(1), 69-76. doi: 10.1016/0277-9536(94)00302-a. 
Northouse, L. L., Kershaw, T., Mood, D., \& Shaughenacker, A. F. (2005). Effects of a family intervention on the quality of life of women with recurrent breast cancer and their family caregivers. Psycho-Oncology, 14(6), 478-491. doi: 10.1002/pon.871.

Northouse, L. L., \& McCorkle, R. (2010). Spouse caregivers of cancer patients. In J. C. Holland, Breitbart, W.S., Jacobsen, P.B., Lederberg, M.S., Loscalzo, M.J., \& McCorkle, R. (Ed.), Psycho-Oncology (pp. 516-521). New York, NY: Oxford University Press.

Northouse, L.L., Mood, D.W., Montie, J.E., Sandler, H.M., Forman, J.D., Hussain, M., Pienta, K.J., Smith, D.C., Sanda, M.G., \& Kershaw, T. (2007). Living with prostate cancer: Patients' and spouses' psychosocial status and quality of life. Journal of Clinical Oncology, 25(27), 4171-4177. doi: 10.1200/JCO.2006.09.6503.

Northouse, L. L., Mood, D.W., Schafenacker, A., Montie, J.E., Sandler, H.M., Forman, J.D., Hussain, M., Pienta, K.J., Smith, D.C. \& Kershaw, T.(2007). Randomized clinical trial of a family intervention for prostate cancer patients and their spouses. Cancer, 110(12), 2809-2818. doi:10.1002/pon.3036.

Northouse, L.L., Mood, D., Templin, T., Mellon, S., \& George, T. (2000). Couples' patterns of adjustment to colon cancer. Social Science \& Medicine, 50(2), 271-284.

Northouse, L. L., Templing, T., Mood, D., \& Oberst, M. (1998). Couples' adjustment to breast cancer and benign breast disease: A longitudinal analysis. Psycho-Oncology, 7(1), 37-48. doi: 10.1002/(sici)1099-1611(199801/02)7:1<37::aid-pon314>3.0.co;2\#. 
Norton, T.R., \& Manne, S.L. (2007). Support concordance among couples coping with cancer: relationship, individual, and situational factors. Journal of Social and Personal Relationships, 24(5), 675-692. doi: 10.1177/0265407507081454.

O'Brien, K., Roe, B., Low, C., Deyn, L., \& Rogers, S.N. (2012). An exploration of the perceived changes in intimacy of patients' relationships following head and neck cancer. Journal of Clininal Nursing, 21(17-18), 2499-2508. doi: 10.1111/j.13652702.2012.04162.x.

O'Brien, T. B., \& DeLongis, A. (1996). The interactional context of problem-, emotion-, and relationship-focused coping: The role of the Big Five personality factors. Journal of Personality, 64, 775-813. doi: 10.1111/j.1467-6494.1996.tb00944.x.

O'Brien, T. B., \& DeLongis, A. (1997). Coping with chronic stress: An interpersonal perspective. In B. H. Gottlieb (Ed.), Coping with chronic stress (pp. 161-190). New York: Plenum Press.

O'Brien, T. B., DeLongis, A., Pomaki, G., Puterman, E., \& Zwicker, A. (2009). Couples coping with stress: The role of emapthic responding. European Psychologist, 14, 18-28. doi: http://dx.doi.org/10.1027/1016-9040.14.1.18.

Olson, D.H. (1986). Circumplex Model VII: Validation studies and FACES III. Family Process, 25(3), 337-351. doi: 10.1111/j.1545-5300.1986.00337.x.

Olson, D. H., Fournier, D. G. and Druckman, J. M. (1986). Counselor's Manual for PREPARE-ENRICH. (rev. ed.), Minneapolis, MN: PREPARE-ENRICH, Inc.

Orbuch, T.L., Veroff, J., Hassan, H., \& Horrocks, J. (2002). Who will divorce: A 14-year longitudinal study of black couples and white couples. Journal of Social and Personal Relationships, 19(2), 179-202. doi: 10.1177/0265407502192002. 
Orbuch, T. L., Veroff, J., \& Hunter, A. G. (1999). Black couples, white couples: The early years of marriage. In E.M. Hetherington (Ed.), Coping with Divorce, SingleParenting and Remarriage (pp. 23-46). Hillsdale, NJ. Erlbaum.

O'Shaughnessy, P.K., Ireland,C., Pelentsov, L., Thomas, L.A., \& Esterman, A.J. (2013). Impaired sexual function and prostate cancer: a mixed method investigation into the experiences of men and their partners. Journal of Clinical Nursing, 22 (23-24), 3492-3502. doi: 10.1111/jocn.12190.

Osse, B.H., Vernooij-Dassen, M.J., Schade, E., \& Grol, R.P. (2006). Problems experienced by the informal caregivers of cancer patients and their needs for support. Cancer Nursing, 29(5), 378-388.

Overcash, J., Extermann, M., Parr, J., Perry, J., \& Balducci, L. (2001). Validity and reliability of the FACT-G scale for use in the older person with cancer. American Journal of Clinical Oncology, 24(6), 591-596.

Pakenham, K. I. (1998). Couple coping and adjustment to multiple sclerosis in care receiver-carer dyads. Family Relations, 47(3), 269-277.

Panjari, M., Bell, R.J., Burney, S., Bell, S., McMurrick, P.J., \& Davis, S.R. (2012). Sexual function, incontinence, and wellbeing in women after rectal cancer. A Review of the Evidence. Journal of Sexual Medicine, 9(11), 2749-2758. doi:10.1111/j.1743-6109.2012.02894.x.

Papp, L. M., \& Witt, N. L. (2010). Romantic partners' individual coping strategies and dyadic coping: Implications for relationship functioning. Journal of Family Psychology, 24(5), 551-559. doi:10. 1037/a0020836. 
Park, B., Lee, S., Lee, A. R., Lee, K.H., \& Hwang, S.Y. (2011). Quality of life differences between younger and older breast cancer patients. Journal of Breast Cancer, 14(2), 112-118. doi: 10.4048/jbc.2011.14.2.112.

Park, E.O., \& Schumacher, K.L. (2013). The state of the science of family caregiver-care receiver mutuality: A systematic review. Nursing Inquiry, 21(2), 140-152. doi: 10.1111/nin.12032.

Pasick, R.J., Barker, J.C., Otero-Sabogal, R., Burk, N.J., Joseph, G., Guerra, C. (2009). Intention, subjective norms, and cancer screening in the context of relational culture. Health Education \& Behavior, 36(5 Suppl), 91S-110S. doi: 10.1177/1090198109338919.

Pasipanodya, E. C., Parrish, B. P., Laurenceau, J. P., Cohen, L. H., Siegel, S. D., Graber, E. C., \& Belcher, A. J. (2012). Social constraints on disclosure predict daily wellbeing in couples coping with early-stage breast cancer. Journal of Family Psychology, 26(4), 661-667. doi: 10.1037/a0028655.

Paterson, C.L., Lengacher, C.A., Donovan, K.A., Kip, K.E., \& Tofthagen, C.S. (2016). Body image in younger breast cancer survivors: A Systematic Review. Cancer Nursing, 39(1), E39-58. doi:10.1097/NCC.0000000000000251.

Patterson, J. M., \& Garwick, A.W. (1994). The impact of chronic illness on families: A family systems perspective. Annals of Behavioral Medicine, 16, 131-142.

Perez, M. A., Skinner, E.C., \& Meyerowtiz, B.E. (2002). Sexuality and intimacy following radical prostatectomy: patient and partner perspectives. Health Psychology, 21(3), 288-293. doi 10.1037//0278-6133.21.3.288. 
Perz, J., Ussher, J.M., Butow, P., \& Wain, G. (2011). Gender differences in cancer carer psychological distress: an analysis of moderators and mediators. European Journal of Cancer Care, 20(5), 610-619. doi: 10.1111/j.1365-2354.2011.01257.x.

Peterson, G. W., \& Bush, K.R. (2013). Handbook of Marriage and the Family (3rd ed.). New York: Springer.

Picard, L., Dumont, S., Gagnon, P., \& Lessard, G. (2005). Coping strategies among couples adjusting to primary breast cancer. Journal of Psychosocial Oncology, 23, 115-135. doi:10.1300/J077v23n02_08.

Pietromonaco, P.R., Feldman Barrett, L., \& Powers, S.I. (2006). Adult Attachment Theory and Affective Reactivity and Regulation. In D.K. Snyder, J.A. Simpson \& J.N. Hughes (eds). Emotion Regulation in Families: Pathways to Dysfunction and Health . Washington, D.C.: American Psychological Association. Available at: http://works.bepress.com/sally_powers/6/.

Pietromonaco, P.R., Uchino, B., \& Dunkel-Schetter, C. (2013). Close relationships processes and health: Implications of Attachment Theory for health and diseases. Health Psychology, 32(5), 499-513. doi: 10.1037/a0029349.

Pinto, A.C. (2013). Sexuality and breast cancer: prime time for young patients. Journal of Thoracic Disease, 5(1), S81-S86. doi:10.3978/j.issn.2072-1439.2013.05.235.

Pistrang, N. \& Baker., C. (1995). The partner relationship in psychological response to breast cancer. Social Science \& Medicine, 40(6), 789-797. doi:10.1016/02779536(94)00136-h. 
Pitcheathly, C., \& Maguire, P. (2003). The psychological impact of cancer on patients' partners and other key relatives: a review. European Journal of Cancer, 39(11), 1517-1524. doi: http://dx.doi.org/10.1016/s0959-8049(03)00309-5.

Ponzetti, J.J. Jr. (2016). Evidence-based approaches to relationship and marriage education. New York, NY: Routledge.

Porter, L. S., Keefe, F. J., Hurwitz, H., \& Faber, M. (2005). Disclosure between patients with gastrointestinal cancer and their spouses. Psycho-Oncology, 14, 1030-1042. doi:10.1002/pon.915.

Preacher, K.J., \& Hayes, A.F. (2008). Asymptotic and resampling strategies for assessing and comparing indirect effects in multiple mediator models. Behavior Research Methods, 40(3), 879-891. doi: 10.3758/BRM.40.3.879.

Primono J., Yates, B. C., \& Woods N.F. (1990). Social support for women during chronic illness: the relationships among sources and types to adjustment. Research in Nursing and Health, 13(3), 153-161. doi: 10.1002/nur.4770130304.

Qualls, S. H. (1997). Aging families: The personal, permanent group. Group, 21, 180190.

Ramsey, S., Blough, D., Kirchhoff, A., Kreizenbeck, K., Fedorenko, C., Snell, K., Newcomb, P., Hollingworth, W., \& Overstreet, K. (2013). Washington State cancer patients found to be at greater risk for bankruptcy than people without a cancer diagnosis. Health Affairs, 32(6), 1143-1152. doi:10.1377/hlthaff.2012.1263.

Ramsey, S.D., Bansal, A., Fedorenko, C.R., Blough, D.K. Overstreet, K.A., Shankaran, V., \& Newcomb, P. (2016). Health services and outcomes: financial insolvency as a risk factor for early mortality among patients with cancer. Journal of Clinical 
Oncology. Published online before print January 25, 2016, doi:

10.1200/JCO.2015.64.6620.

Randall, A.K., \& Bodenmann, G. (2009). The role of stress on close relationships and marital satisfaction. Clinical Psychology Review, 29(2), 105-115. doi: 10.1016/j.cpr.2008.10.004.

Raykov, T., \& Marcuulides, G.A. (2006). A First Course in Structural Equation Modeling. Mahwah, NJ: Lawrence Erlbaum Associates.

Regan, T. W., Lambert, S.D., Girgis, A., Kelly, B., Kayser, K., \& Turner, J. (2012). Do Couple-based Interventions make a difference for couples affected by cancer?. A Systematic Review. BMC Cancer, 12. doi: http://www.biomedcentral.com/14712407/12/279.

Regan, T, Lambert, S.D., \& Kelly, B. (2013). Uptake and attrition in couple-based interventions for cancer: perspectives from the literature. Psycho-Oncology, 22(12), 2639-2647. doi: 10.1002/pon.3342.

Regan, T.W., Lambert, S.D., Kelly, B., Falconier, M., Kissane, D., \& Levesque, J.V. (2015a). Couples coping with cancer: Exploration of theoretical framewoks from dyadic studies. Psycho-Oncology, 24(12).1605-1617. doi: 10.1002/pon.3854.

Regan, T.W., Lambert, S.D., Kelly, B , McElduff, P, Girgis, A;, Kayser, K; \& Turner, J. (2014). Cross-sectional relationship between dyadic coping and anxiety, depression, and relationship satisfaction for patients with prostate cancer and their spouses. Patient Education and Counselling, 96(1), 120-127. doi: 10.1016/j.pec.2014.04.010. 
Regan, T., Levesque, J.V., Lambert, S.D., \& Kelly, B. (2015b) A qualitative investigation of health care professionals', patients' and partners' views on psychosocial issues and related interventions for couples coping with cancer. PLOS ONE 10(7): e0133837. doi:10.1371/journal.pone.0133837.

Reichers, E. A. (2004). Including partners into the diagnosis of prostate cancer: A review of the literature to provide a model of care. Urology Nursing, 24(1), 22-38.

Reidy, M., \& Denieffe, S. (2014). Breast cancer in younger women from diverse cultural backgrounds. British Journal of Nursing, 23(4), S19-22, S24-S26. doi:

10.12968/bjon.2014.23.sup2.s19.

Reis, N., Beji, N. K., \& Coskun, A. (2010). Quality of life and sexual functioning in gynecological cancer patients: results from quantitative and qualitative data. European Journal of Oncology Nursing, 14(2), 137-146. doi: 10.1016/j.ejon.2009.09.004.

Revenson, T. A. (1994). Social support and marital coping with chronic illness. Annals of Behavioral Medicine, 16(2), 122-130.

Revenson, T. A. (2003). Scenes from a marriage: Examining supportand marital coping with chronic illness. In J. Suls \& K. Wallston (Eds.), Social psychological foundations of health and illness (pp. 530-559). Oxford, England: Blackwell Publishing.

Revenson, T. A., Abraido-Lanza, A. F., Majerovitz, S. D., \& Jordan, C. (2005). Couples coping with chronic illness: What's gender got to do with it? In T. A. Revenson, K. Kayser, \& G. Bodenmann (Eds.), Couples coping with stress: Emerging 
perspectives on dyadic coping (pp. 121-136). Washington, DC: American

Psychological Association

Revenson, T. A., \& DeLongis, A. (2011). Couples coping with Chronic Illness. In S.

Folkman (Ed.), The Oxford Handbook of Stress, Health and Coping New York, NY: Oxford University Press.

Revenson, T. A., Kayser, K., \& Bodenmann, G. (2005). Couples Coping with Stress:

Emerging Perspectives on Dyadic Coping. Washington D.C.: American

Psychological Association.

Revenson, T., \& Lepore, S.J. (2012). Coping in Social Context. In A.Baum,.T.A.

Revenson \& J.Singer (Eds.). Handbook of Health Psychology. Second Edition (pp.

193-217), New York, NY: Taylor \& Francis Group.

Revenson, T.A., \& Pranikoff, J.R.(2005). A contextual approach to treatment decision making among breast cancer survivors. Health Psychology, 24(4 Suppl),S93-S98. http://dx.doi.org/10.1037/0278-6133.24.4.S93.

Reynolds, J. S., \& Perrin, N.A. (2004). Mismatches in social support and psychosocial adjustment to breast cancer. Health Psychology, 23(4), 425-430.

http://dx.doi.org/10.1037/0278-6133.23.4.425

Ries, L.A.G., Young, J.L., Keel, G.E., Eisner, M.P., Lin, Y.D., \& Horner, M-J.(2007). SEER Survival Monograph: Cancer Survival Among Adults: U.S. SEER Program, 1988-2001, Patient and Tumor Characteristics. National Cancer Institute, SEER Program, NIH. 
Rivers, D., August, E.M., Sehovic, I., Lee Green, B., \& Quinn, G.P. (2013). A systematic review of the factors influencing African Americans' participation in cancer clinical trials. Contemporary Clinical Trials, 35 (2), 13-32. doi: 10.1016/j.cct.2013.03.007.

Robbins, M.L., López, A.M., Weihs, K.L., \& Mehl, M.R. (2014). Cancer conversations in context: Naturalistic observation of couples coping with breast cancer. Journal of Family Psychoogy, 28(3), 380-390. doi: 10.1037/a0036458.

Roberts, K. J., Lepore, S. J., \& Helgeson, V. S. (2006). Social-cognitive correlates of adjustment to prostate cancer. Psycho-Oncology, 15(3), 183-192. doi: 10.1002/pon.934.

Rodin, G., Walsh, A., Zimmerman, C., Gagliese, L., Jones, J., Sheperd, F.A., et al. (2007). The contribution of attachment security and social support to depressive symptoms in patients with metastatic cancer. Psycho-Oncology, 16(12), 1080-1091. doi:10.1002/pon.1186.

Rogers, S.J. (2004). Dollars, dependency, and divorce: Four perspectives on the role of wives' income. Journal of Marriage and Family, 66(1), :59-74. doi: 10.1111/j.17413737.2004.00005.x

Rogers, L. E., \& Escudero, V. (2004). Relational Communication: An Interactional Perspective to the Study of Process and Form. Mahwah NJ: Lawrence Erlbaun Associates.

Rogoff, B. (1998). Cognition as a collaborative process, in D. Kuhn and R. S. Siegler (Eds.), Handbook of Child Psychology, Vol2, 5th edition. New York: John Wiley, pp. 679-744. 
Rogoff, B. (2003). The cultural nature of human development. Oxford: Oxford University Press.

Rohrbaugh, M.J., Cranford, J.A., Shoham, V., Nicklas, J.M., Sonnega, J. \& Coyne, J.C. (2002). Couples coping with congestive heart failure: Role and gender differences in psychological distress. Journal of Family Psychology, 16(1), 3-13. http://dx.doi.org/10.1037/0893-3200.16.1.3,

Rolland, J. S. (1994). In Sickness and in health: The impact of illness on couple's relationships. Journal of Marital and Family Therapy, 20(4), 327-347. DOI: 10.1111/j.1752-0606.1994.tb00125.x.

Romero, C., Linsday, J. E., Dalton, W. T., Nelson, D. V., \& Friedman, L. C. (2008). Husbands' perceptions of wives' adjustment to breast cancer: The impact on wives mood. Psycho Oncology, 17(3), 237-243. doi: 10.1002/pon.1224.

Rosenberg, S.M., Tamimi, R.M., Gelber, S., Ruddy, K.J., Kereakoglow, S, Borges, V.F., Corne, S.E., Schapira, L., Winer, E., \& Partridge, A.H. (2013). Body image in recently diagnosed young women with early breast cancer. Psycho-Oncology, 22(8), 1849-1855. doi: 10.1002/pon.3221.

Rothermund, K., \& Brandstadter, J. (2003). Coping with deficits and losses in later life: From compesatory action to accomodation. Psychology \& Aging, 18, 896-905. http://dx.doi.org/10.1037/0882-7974.18.4.896.

Rottmann, N., Hansen, D. G., Larsen, P. V., Nicolaisen, A., Flyger, H., Johansen, C., \& Hagedoorn, M. (2015). Dyadic Coping within couples dealing with breast cancer: A longitudinal, population-based study. Health Psychology, 34(5), 486-495. 10.1037/hea0000218. 
Rowland, J.H., \& Massie, M.J. (2010). Breast Cancer. In J.C. Holland, W.S. Breitbart, P.B. Jacobsen, M.S. Lederberg, M.J. Loscalzo, \& R. McCorkle (eds.). PsychoOncology (177-186). .New York, NY: Oxford University Press

Ruddy, K.J., Greaney, M.L., Sprunck-Harrild, K., Meyer ,M.E., Emmons, K.M., \& Partridge, A.H.(2013). Young women with breast cancer: A focus group study of unmet needs. Journal of Adolescent and Young Adult Oncology, 2(4), 153-160. doi:10.1089/jayao.2013.0014.

Ruddy, K.J. \& Partridge, A.H. (2012). Fertility (male and female) and Menopause. Journal of Clinical Oncology. Published online before print September 24, 2012, doi: 10.1200/JCO.2012.42.1966.

Ruffieux, M. Nussbeck, F. N. \& Bodenmann, G. (2014). Long-term prediction of relationship satisfaction and stability by stress, coping, communication, and wellbeing. Journal of Divorce \& Remarriage, 55, 485- 501. doi:10.1080/10502556.2014.931767.

Safarinejad, M.R., Shafiei, N,, Safarinejad, S. (2013). Quality of life and sexual functioning in young women with early-stage breast cancer 1 year after lumpectomy. Psycho-Oncology, 22(6), 1242-1248. doi: 10.1002/pon.3130.

Saita, E. (2009). Psico-Oncologia: Una Prospettiva Relazionale. Milano: Edizioni Unicopli.

Saita, E. (2011). Pensare alla Salute e alla Malattia. Legami tra Mente, Corpo e Contesto di Appartenenza. Milano: EDUCatt. 
Saita, E., Acquati, C., \& Kayser, K. (2015). Coping with early stage breast cancer:

Examining the influence of personality traits and interpersonal closeness. Frontiers in Psychology: Psychology for Clinical Settings, doi: 10.3389/fpsyg.2015.00088.

Saita. E., Cigoli, V., \& Margola, D. (2009). L'intervento con il gruppo: Una metodologia innovativa di cura. In E. Saita (ed). Psico-Oncologia: Una Prospettiva Relazionale.(pp. 214-235). Milano: Edizioni Unicopli.

Salsman, J., Pearman, T., \& Cella, D. (2013). Quality of Life. In B.I. Carr \& J. Steel (eds.) Psychological Aspects of Cancer. A Guide to Emotional Consequences of Cancer, Their Causes and Their Management (pp. 255-278). New York: Springer

Salsman, J.M., Sofia F. Garcia, S.F., Yanez, B., Stacy D. Sanford, S.D., Mallory A. Snyder, M.A., \& Victorson, D. (2014). Physical, emotional, and social health differences between post-treatment young adults with cancer and matched healthy controls. Cancer, 120(15), 2247-2254. doi: 10.1002/cncr.28739.

Sanders, S. L., Bantum, E. O., Owen, J. E., Thornton, A. A., \& Stanton, A. L. (2010). Supportive care needs in patients with lung cancer. Psycho-Oncology, 19(5), 480489. doi: 10.1002/pon.1577.

Sanders, S., Pedro, L.W., Bantum, E.O., \& Galbraith, M.E. (2006). Couples surviving prostate cancer: long-term intimacy needs and concerns following treatment. Clinical Journal of Oncology Nursing, 10(4), 503-508. doi: 10.1188/06.cjon.503508.

Sandgren, A. K., Mullens, A.B., Erickson, S.C., Romanek, K.M, \& McCaul, K.D. (2004). Confidant and breast cancer patient reports of quality of life. Quality of Life Research, 13(1), 155-160. 
Sanford, S.D., Zhao, F., Salsman, J.M., Victor T. Chang, V.T., Wagner, L.I., \& Fisch, M.J. (2014). Symptom burden among young adults with breast or colorectal cancer. Cancer, 120(15), 2255-2263. doi: 10.1002/cncr.28297.

Sassler, S. (2010). Partnering across the life course: Sex, relationships, and mate selection. Journal of Marriage and Family, 72, 557-575. doi: 10.1111/j.17413737.2010.00718.x.

Scabini, E., \& Cigoli, V. (2000). Il famigliare. Legami, simboli e trnsizioni. Milano: Raffaello Cortina.

Scabini, E., \& Iafrate, R. (2003). Psicologia del legami familiari. Bologna: Il Mulino. Scabini, E., \& Rossi, G. (2012). Family transitions and families in transitions. Milano: Vita\&Pensiero.

Schaer, M., Bodenmann, G., \& Klink, T. (2008). Balancing Work and Relationship: Couples Coping Enhancement Training (CCET) in the Workplace. Applied Psychology, 57(s1), 71-89. doi:10.1111/j.1464-0597.2008.00355.x.

Schang, C. A., Ganz, P.A., Polinsky, M.L., Fred, C., Hirji, K., \& Petersen, L. (1993). Characteristics of women at risk for psychological distress in the year after breast cancer. Journal of Clinical Oncology, 11(4), 783-793.

Schmitz, G. S. (1998). Cardiac patients and their spouses: Towards a perspective of dyadic coping. In R. Schwarzer (Ed.). Advances in Health Psychology Research. Berlin, Germany: Free University. CD-ROM Volume.

Schneewind, K. A., \& Kruse, J. (2002). Die Paarklimaskalen(PKS). Manual [Atmosphere of couple relation Scale. Manual]. Bern, Switzerland: Huber 
Schneewind. K.A., \& Gerhard, A.-K. (2002). Relationship personality, conflict resolution, and marital satisfaction in the first 5 years of marriage. Family Relations, 51(1), 63-71.doi: 10.1111/j.1741-3729.2002.00063.x

Schokker, M. C., Stuive, I., Bouma, J., Keers, J. C., Links, T. P., Wolffen-Buttel, B. H. R., Sanderman, R., \& Hagedoorn, M. (2010). Support behavior and relationship satisfaction in couples dealing with diabetes: Main and moderating effects. Journal of Family Psychology, 24,578-586. doi:10.1037/a0021009.

Schover, L. R., Montague, D.K., \& Lakin, M.M. (1997). Sexual problems. In S. H. V.T. DeVita, \& S.A. Rosenberg (Ed.), Cancer: principles and practice of oncology (5th Edition ed., pp. 2857-2872). Philadelphia: Lippincott-Raven.

Schover, L.R., van der Kaaij, M., van Dorst, E., Creutzberg, C., Huyghe, E., \& Kiserud, C.(2014). Sexual dysfunction and infertility as late effects of cancer treatment. European Journal of Cancer - Supplements, 12(1):41-53. doi:

10.1016/j.ejcsup.2014.03.004.

Schroevers, M.J., Ranchor, A.V., \& Sanderman, R. (2004). The role of age at the onset of cancer in relation to survivors' long-term adjustment: A controlled comparison over an eight-year period. Psycho-Oncology, 13(10), 740-752. doi: 10.1002/pon.780.

Schulz, U., \& Schwarzer, R. (2004). Partnerschaftliche Bewältigung einer Krebserkrankung [Dyadic coping with cancer]. In P. Buchwald, S. E. Hobfoll \& C. Schwarzer (Eds.), Stress gemeinsam bewältigen (pp. 121-138). Göttingen, Germany: Hogrefe.

Schwarzer, C., \& Busch, B. (2005). Dyadic coping with retirement in elderly couples. International Association of Applied Psychology Newsletter, 17, 13-23. 
Scott, J. L., Halford, W. K., \& Ward, B. G. (2004). United we stand? The effects of a couple-coping intervention on adjustment to early stage breast or gynaecological cancer. Journal of Consulting and Clinical Psychology, 72(6), 1122-1135. doi: 10.1037/0022-006x.72.6.1122.

Scott, J. L., \& Kayser, K. (2009a). A review of couple-based interventions for enhancing women's sexual adjustment and body image after cancer. Cancer, 15(1), 48-56. doi: 10.1097/PPO.0b013e31819585df.

Scott, J. L., \& Kayser, K. (2009b). Promuovere il coping nei confronti del cancro: Un approccio centrato sulla coppia. In E. Saita (Ed.), Psico-Oncologia: Una Prospettiva Relazionale (pp. 155-180). Milan: Unicopli.

Segrin, C., \& Badger, T.A. (2014). Psychological and physical distress are interdependent in breast cancer survivors and their partners. Psychology, Health \& Medicine, 19(6), doi:10.1080/13548506.2013.871304.

Segrin, C., Badger, T., Meek, P., Lopez, A. M., Bonham, E.,\& Sieger, A. (2005). Dyadic interdependence on affect and quality of life trajectories among women with breast cancer and their partners. Journal of Social and Personal Relationships, 22(5), 673689. doi: $10.1177 / 0265407505056443$.

Selye, H. (1956). The stress of life. New York: McGraw-Hill.

Shahi, V., Lapid, M. I., Kung, S., Atherton, P. J., Sloan, J. A., Clark, M. M., \& Rummans, T. A. (2014). Do age and quality of life of patients with cancer influence quality of life of the caregiver?. Journal of Geriatric Oncology, 5(3), 331-336. 10.1016/j.jgo.2014.03.003. 
Sharp, L., Carsin, A.E., \& Timmons, A. (2012). Associations between cancer-related financial stress and strain and psychological well-being among individuals living with cancer. Psycho-oncology, 22(4), 745-755. doi: 10.1002/pon.3055.

Shi, L., Lebrun, L.A., Zhu, J, \& Tsai, J. (2011). Cancer screening among Racial/Ethnic and insurance groups in the United States: A comparison of disparities in 2000 and 2008. Journal of Health Care for the Poor and Underserved, 22(3), 945-961. doi: 10.1353/hpu.2011.0079.

Shiota, M.N., \& Levenson, RW.(2007). Birds of a feather don't always fly farthest: similarity in Big Five personality predicts more negative marital satisfaction trajectories in long-term marriages. Psychology and Aging, 22(4),666-765. doi: 10.1037/0882-7974.22.4.666.

Shortt, J. W., Capaldi, D. M., Kim, H. K., \& Tiberio, S. S. (2013). The interplay between interpersonal stress and psychological intimate partner violence over time for young at-risk couples. Journal of Youth and Adolescence, 42, 619-632.doi: 10.1007/s 10964-013-9911-y.

Shrout, P.E., \& Bolger, N. (2002). Mediation in experimental and non experimental studies: New procedures and recommendations. Psychological Methods, 7(4), 422445. doi: 10.1037//1082-989X.7.4.422.

Siegel, D.L. (2012). The Developing Mind, Second Edition: How relationships and the brain interact to shape who we are. New York, NY: Guilford Press.

Siegel, R., Miller, K., \& Jemal, A. (2015). Cancer Statistics, 2015. CA: Cancer Journal for Clinicians, 65 (1), 5-29. doi: 10.3322/caac.21254. 
Siegel, R., Naishadham, D., \& Jemal, A. (2013). Cancer statistics, 2013. CA: A Cancer Journal for Clinicians, 63(1), 11-30. doi: 10.3322/caac.21166.

Simeone, S., Savini, S., Torino, F., Vellone, E., \& Alvaro, R. (2014). Mutuality in caregiving: a literature review. Professioni Infermieristiche, 67(3), 155-165. doi: 10.7429/pi.2014.673155.

Sjolander, C., Rolander, B., Järhult, J., Mårtensson, J., \& Ahlstrom, G. (2012). Healthrelated quality of life in family members of patients with an advanced cancer diagnosis: a one-year prospective study. Health and Quality of Life Outcomes, 10(1). doi: 10.1186/1477-7525-10-89.

Skerrett, K. (1998). Couple adjustment to the experience of breast cancer. Families Systems and Health, 16(3), 281-298. http://dx.doi.org/10.1037/h0089855.

Skerrett, K., \& Fergus, K. (2015). Couple resilience. New York, NY: Springer

Smith, S.R., \& Hamon, R.R. (2012). Exploring Family Theories (Third Edition). New York, NY: Oxford University Press

Snippe, E., Maters, G. A., Wempe, J.B., Hagedoorn, M., Sanderman, R. (2012). Discrepancies between patients' and partners' perceptions of unsupportive behavior in chronic obstructive pulmonary disease. Journal of Family Psychology, 26(3), 464-469. doi: 10.1037/a0028333.

So, W.K., Choi, K.C., Chan, C.W., \& Chair SY. (2011). Age-related differences in the quality of life of Chinese women undergoing adjuvant therapy for breast cancer. Research in Gerontological Nursing, 4(1), 19-26. doi:10.3928/1940492120101201-01. 
So, W.K., Marsh, G., Ling, W.M., Leung, F.Y., Lo, J.C., et al. (2009). The symptom cluster of fatigue, pain, anxiety, and depression and the effect on the quality of life of women receiving treatment for breast cancer: a multicenter study. Oncology Nursing Forum, 36(4), E205-E214. doi:10.1188/09.ONF.E205-E214.

Solano, L. (2001). Tra mente e corpo. Milano: Cortina.

Song, J.I., Shin, D.W., Choi, J.Y., Kang, J., Baik, Y.J., Mo, H., Park, M.H., Choi, S.E., Kwak, J.H., \& Kim, E.J.(2011). Quality of life and mental health in family caregivers of patients with terminal cancer. Supportive Care in Cancer, 19(10), 1519-1526. doi:10.1007/s00520-010-0977-8.

Song, L., Bensen, J.T., Zimmer, C., Sleath, B., Blackard, B., Elizabeth Fontham, E., Su, L.J., Christine S. Brennan, C.S., Mohler, J.L., \& Mishel, M. (2013). Patient-health care provider communication among patients with newly diagnosed prostate cancer: Findings from a population-based survey. Patient Education and Counseling, 91(1): 79-84. doi: 10.1016/j.pec.2012.12.002.

Song, L., Hamilton, J.B., \& Moore, A.D. (2012). Patient-healthcare provider communication: perspectives of African American cancer patients. Health Psychology, 31(5), 539-547. doi: 10.1037/a0025334.

Song, L., Northouse, L.L., Braun, T.M., Zhang, L., Cimprich, B., Ronis, D.L., \& Mood, D.W. (2011). Assessing longitudinal quality of life in prostate cancer patients and their spouses: A multilevel modeling approach. Quality of Life Research, 20(3), 371-381. doi: 10.1007/s11136-010-9753-y. 
Song, L., Northouse, L.L., Zhang, L., Braun, T.M., Cimprich, B., Ronis, D.L. et al, (2012). Study of dyadic communication in couples managing prostate cancer: a longitudinal perspective. Psycho-Oncology, 21(1), 72-81. doi: 10.1002/pon.1861

Sormanti, M., \& Kayser K. (2000). Partner support and relationship changes during lifethreatening illness: women's perspectives. Journal of Psychosocial Oncology, 18, 45-66. doi:10.1300/J077v18n03_04.

Spencer, R. (2005). Understanding the mentoring process between adolescents and adults. Youth \& Society, 37(3), 287-315. doi: 10.1177/0743558405278263.

Stanton, A. L., Danoff-Burg, S., Huggins, M.E. (2002). The first year after breast cancer diagnosis: Hope and coping strategies as predictors of adjustment. PsychoOncology, 11(2), 93-102. doi: 10.1002/pon.574.

Stanton, A. L., Revenson, T. A., \& Tennen, H. (2007). Health psychology: Adjustment to chronic disease. Annual Review of Psychology, 58, 565-592. doi:

10.1146/annurev.psych.58.110405.085615.

Stava, C.J., Lopez, A., \& Vassilopoulou-Sellin, R. (2006). Health profiles of younger and older breast cancer survivors. Cancer, 107(8),1752-1759. doi: 10.1002/cncr.22200.

Stenberg, U., Ruland, C.M., \& Miaskowski, C. (2010). Review of the literature on the effects of caring for a patient with cancer. Psycho-Oncology, 19(10), 1013-1025. doi: $10.1002 /$ pon. 1670 .

Sterba, K. R., DeVellis, R.F., Lewis, M.A., DeVellis, B., Jordan, J., \& Baucom, D.H. (2008). Effect of couple illness perception congruence on psychological adjustment in women with rheumatoid arthritis. Health Psychology, 27(2), 221-229. doi: 10.1037/0278-6133.27.2.221. 
Sullivan, H. S. (1940). Conceptions of modern psychology. New York: NY: William Allison White Psychiatric Foundation.

Sullivan, H. S. (1953). The interpersonal theory of psychiatry. New York, NY: Norton.

Sullivan, K.T., Pasch, L.A., Johnson, M.D., \& Bradbury, T.N. (2010). Social support, problem solving, and the longitudinal course of newlywed marriage. Journal of Personality and Social Psychology, 98(4), 631-644. doi: 10.1037/a0017578.

Suls, J., \& Fletcher, B. (1985). The relative efficacy of avoidant and nonavoidant coping strategies: a meta-analysis. Health Psychology, 4(3), 249-288.

http://dx.doi.org/10.1037/0278-6133.4.3.249.

Suls, J., Green, P., Rose, G., Lounsbury, P., \& Gordon, E. . (1997). Hiding worries from one's spouse: Associations between coping via protective buffering and distress in male post-myocardial infarction patients and their wives. Journal of Behavioral Medicine, 20(4), 333-349.

Surbone, A., Zwitter, M., Rajer, M., \& Stiefel, R. (2013). New challenges in communication with cancer patients. New York, NY: Springer.

Tabachnick, B.G., \& Fidell, L.S. (2007). Using Multivariate Statistics. Fifth Edition. Boston, MA: Pearson Allyn \& Bacon.

Taylor, C.L., Badr, H., Lee, J.H. et al. (2008). Lung cancer patients and their spouses: psychological and relationship functioning within 1 month of treatment initiation. Annals of Behavioral Medicine, 36(2):129-140. doi: 10.1007/s12160-008-9062-7.

Tedeschi, R. G., \& Calhoun, L. G. (1995). Trauma \& Transformation: Growing in the Aftermath of Suffering. Thousand Oaks, CA: Sage. 
Tedeschi, R. G. \& Calhoun, L. G. (1996). The Posttraumatic Growth Inventory:

Measuring the Positive Legacy of Trauma. Journal of Traumatic Stress, 9(3), 455471.

Tedeschi, R. G. \& Calhoun, L. G. (1998). Posttraumatic Growth: Positive Changes in the Aftermath of Crisis. Mahwah, NJ: Lawrence Earlbaum Associates.

Tedeschi, R. G., \& Calhoun, L. G. (2004). Posttraumatic growth: Conceptual foundations and empirical evidence. Psychological Inquiry An International Journal for the Advancement of Psychological Theory, 15(1), 1-18. doi:10.1207/s15327965pli1501_01.

Thaler-DeMers, D. (2002). Sexuality, fertility issues, and cancer. Illness, Crisis, \& Loss, 10(1), 27-41. doi: 10.1177/1054137302010001004

Thewes, B., Bell, M. L., Butow, P., Beith, J., Boyle, F., Friedlander, M., \& McLachlan, S. A. (2013). Psychological morbidity and stress but not social factors influence level of fear of cancer recurrence in young women with early breast cancer: Results of a cross-sectional study. Psycho-Oncology, 22(12), 2797-2806. 10.1002/pon.3348.

Thewes, B., Meiser, A., Taylor, K.A., Phillips, S. Pendlebury, A., Capp, A., Dailey, D., Goldstein, D., Baber, R., \& Friedlander, M.L. (2005). Fertility- and menopauserelated information needs of younger women with a diagnosis of early breast cancer. Journal of Clinical Oncology, 23(22), 5155-5165.

Thomson, E. ,Winkler-Dworak, M., \& Kennedy, S. (2013). The standard family life course: An assessment of variability in life course pathways. In:Evans, A. \& Baxter, 
J. (eds.). Negotiating the Life Course: Stability and Change in Life Pathways.

Dordrecht: Springer: 35-52.doi:10.1007/978-90-481-8912-0_3.

Thornton, A. A., \& Perez, M. A. (2006). Posttraumatic growth in prostate cancer survivors and their partners. Psycho-Oncology, 15(4), 285-296. doi: 10.1002/pon.953.

Thornton, A.A., Perez, M.A., \& Meyerowitz, B,E. (2004). Patient and partner quality of life and psychosocial adjustment following radical prostatectomy. Journal of Clinical Psychology in Medical Settings, 11(1), 15-30.

Traa, M.J., Braeken, J., De Vries, J., Roukema, J.A., Gerrit D., Slooter, G.D., Crolla. R., Monique P. M. Borremans, M.P.M., \& Den Oudsten, B.L. (2015a). Sexual, marital, and general life functioning in couples coping with colorectal cancer: a dyadic study across time. Psycho-Oncology, 24(9), 1181-1188. doi: 10.1002/pon.3801.

Traa, M.J., Braeken, J., De Vries, J., Roukema, J.A., Orsini, R.G., \& Den Oudsten, B.L.(2014). Evaluating quality of life and response shift from a couple-based perspective: a study among patients with colorectal cancer and their partners. Quality of Life Research, 24(6), 1431-1441. doi: 10.1007/s11136-014-0872-8. Epub 2014 Nov 28.

Traa, M.J., De Vries, J., Bodenmann, G., \& Den Oudsten, B.L. (2015b). Dyadic coping and relationship functioning in couples coping with cancer: A systematic review. British Journal of Health Psychology, 20(1), 85-114. doi: 10.1111/bjhp.12094.

Traeger, L. Greer, J. A., Fernandez-Robles, C., Temel, J.S., \& Pirl, W.F. (2012). Evidence-based treatment of anxiety in patients with cancer. Journal of Clinical Oncology, 30(11), 1197-1205. doi: 10.1200/JCO.2011.39.5632. 
Tran, T.V. (2009). Developing cross-cultural measurement. NY: Oxford University Press.

Trudeau-Hern, S., \& Daneshpour, M. (2012). Cancer's impact on spousal caregiver health: A qualitative analysis in grounded theory. Contemporary Family Therapy, 34(4), 534-554.doi: 10.1007/s.10591-012-9211-9.

Tuinman, M.A., Van Nuenen, F,M., Hagedoorn, M., \& Hoekstra-Weebers, J.E.. (2015). Distress, problems and referral wish of cancer patients: differences according to relationship status and life phase. Psycho-Oncology. 24(6):699-704. doi: 10.1002/pon.3712.

Upchurch, R., Malcarne, V. L., Hansdottir, I., Greenbergs, H. L., McKinney, A., Agee, G. H., et al. (2003).Coping patterns of couples predict quality of life outcomes in patients with systemic sclerosis and their spouses. Unpublished manuscript

Ussher, J., Perz, J., Gilbert, E., Hawkins, Y., \& Wong, W. (2012). Sexuality and Intimacy in the Context of Cancer. In R. Mohan (Ed.), Topics in Cancer Survivorship, InTech. Available online at http://cdn.intechopen.com/pdfs-wm/26737.pdf.

Ussher,J.M., Perz, J., Gilbert, E. \& Australian Cancer and Sexuality Study Team (2015). Perceived causes and consequences of sexual changes after cancer for women and men: a mixed method study. BMC Cancer, 15, 268. doi: 10.1186/s12885-015-12438.

Vadaparampil, S.T., Hutchins, N.M., \& Quinn, G.P. (2013). Reproductive health in the adolescent and young adult cancer patient: an innovative training program for oncology nurses. Journal of Cancer Education, 28(1), 197-208. doi: 10.1007/s13187-012-0435-z. 
Vaillant, G. (1977). Adaptation to life: How the best and the brightest came of age.

Boston: Little Brown

Vaillant, G. (1993). The wisdom of the Ego. Cambridge, MA: Harvard University Press

Valdivieso, M., Kujawa, A.M., Jones, T., \& Baker, L.H. (2012). Cancer survivors in the United States: A review of the literature and a call to action. International Journal of Medical Sciences, 9(2), 163-173.doi:10.7150/ijms.3827.

van Steenbergen, E.F., Kluwer, E.S., \& Karney, B.R. (2011). Workload and the trajectory of marital satisfaction in newlyweds: job satisfaction, gender, and parental status as moderators. Journal of Family Psychology, 25(3):345-55. doi: 10.1037/a0023653.

Vanlemmens, L., Christophe, V., Fournier, E., Dauchy, S., Boinon, D., Toudic-Emily, F., Duffor, B., Machavoine, J-L., Reich, M., Bonneterre, J., Kaci, F.A., \& Antoine, P. (2012a). The quality of life of young women with nonmetastatic braest cancer and their partners': Specific needs require development of scientific questionnaires for each of them. The Breast Journal, 18(2), 182-184. doi: 10.1111/j.15244741.2011.01218.x.

Vanlemmens, L., Fournier, E., Boinon, D., Machavoine, J.L., \& Christophe, V. (2012b). Quality of life of young women with early breast cancer and their partners: Specific needs result in the necessity of development of specific questionnaires for the patients and the partner. Bulletin du Cancer, 99(6), 685-691. doi: 10.1684/bdc.2012.1598.

Varner, A. (2015). Caregivers of Cancer Patients. In G. Crist, C. Messner, \& L. Behar (eds.) Handbook of Oncology Social Work. Psychosocial Care for People with Cancer (pp. 385-390). New York, NY: Oxford University Press. 
Vedes, A., Nussbeck, F., \& Bodenmann, G (2015). A life cycle perspective on stress, dyadic coping and couples' well-being. Paper presented at the 9yh Workshopkongress für Klinische Psychologie und Psychoterapie/33. Symposim der Fachgruppe Klinische Psychologie und Psychotherapie der DGPs,Dresden, Germany

Vedes, A., Nussbeck, F., Bodenmann, G., Lind, W., \& Randall, C. (2013). The role of we-ness in mediating the association between dyadic coping and relationship satisfaction (manuscript re-submitted to the Journal of Social and Personal Relationships). Paper presented at the Sixth International Meeting on Stress \& Coping. Couples Coping with Cancer-Related Stress: Translating Research to Practice, June 21-22, Louisville, KY

Vedes, A.; Nussbeck, F.W., Bodenmann, G., Lind, W., \& Ferreira, A. (2013).

Psychometric properties and validity of the Dyadic Coping Inventory in Portuguese. Swiss Journal of Psychology, 72(3), 149-157. http://dx.doi.org/10.1024/14210185/a000108

Ventura, E.E., Ganz, P.A., Bower, J.E., Abascal, L., Petersen, L., Stanton, A.L., \& Crespi, C.M. (2013). Barriers to physical activity and healthy eating in young breast cancer survivors: modifiable risk factors and associations with body mass index.

Breast Cancer Research and Treatment, 142(2), 423-433. doi: 10.1007/s10549-0132749-x

Verhaeghen, P., \& Hertzog, C. (2014). The Oxford Handbook of Emotion, Social Cognition, and Problem Solving in Adulthood. New York, NY: Oxford University Press. 
Verhofstadt, L.L., Ickes, W., \& Buysse, A. (2010). Empathic accuracy and support provision in marriage. In K. Sullivan and J. Davila (Eds.), Support processes in intimate relationships (pp. 71-88). New York, NY: Oxford University Press.

Veroff, J. (1999). Marital commitment in the early years of marriage. In W.H. Jones \& J.M. Adams (Eds.), Handbook of Interpersonal Commitment and Relationship Stability. (pp. 149-162). New York: Plenum.

Vilchinsky, N., Liat Haze-Filderman,L.H., Morton Leibowitz, M., Reges, O., Khaskia, A., \& Mosseri, M. (2010). Spousal support and cardiac patients' distress: The moderating role of attachment orientation. Journal of Family Psychology, 24(4), 508-512. . http://dx.doi.org/10.1037/a0020009.

von Bertalanffy, L. (1969). General System Theory. New York, NY: George Braziller. von Bertalanffy, L. (1975). Perspectives on General Systems Theory - Scientific and Philosophical Studies. (E. Taschdigian \& M. von Bertalanffy, Eds.) New York: Braziller.

Vygotsky, L. S. (1987). Thinking and Speech. In R.W. Rieber \& A.S. Carton (Eds.) The collected works of L. S. Vygotsky, Vol. 1, Problems of general psychology (N. Minick, Trans.) (239-285). New York: Plenum Press.

Wadhwa, D., Burman, D., Swami, N., Rodin, G., Lo, C., \& Zimmermann, C. (2013). Quality of life and mental health in caregivers of outpatients with advanced cancer. Psycho-Oncology, 22(2), 403-410. doi: 10.1002/pon.2104.

Wadsworth, M.E., Gudmundsen, G. R., Raviv, T., Ahlkvist, J.A., McIntosh, D.N., Kline, G.H., et al. (2004). Coping with terrorism: Age and gender differences in effortful 
and involuntary responses to September 11th. Applied Developmental Science, 8(3), 143-157. doi:10.1207/s1532480xads0803_4.

Wagner, C.D., Tanmoy Das, L., Bigatti, S.M., \& Storniolo, A.M. (2011). Characterizing burden, caregiving benefits, and psychological distress of husbands of breast cancer patients during treatment and beyond. Cancer Nursing, 34(4), E21-E30. doi: 10.1097/NCC.0b013e31820251f5.

Walen, H. R., \& Lachman, M.E. (2000). Social support and strain from partner, family, and friends: Costs and benefits for men and women in adulthood. Journal of Social and Personal Relationships, 17(1), 5-30. doi: 10.1177/0265407500171001.

Walker, M. (2005). Critical thinking: Challenging developmental myths, stigmas, and stereotypes. In D. Comstock (Ed.), Diversity and development: Critical contexts that shape our lives and relationships (pp. 47-67). Belmont, CA: Brooks Cole.

Walker, L. M., \& Robinson, J. W. (2010). The unique needs of couples experiencing androgen deprivation therapy for prostate cancer. Journal of Sex \& Marital Therapy, 36(2), 154-165. doi: 10.1080/00926230903554552

Walsh, S. R., Manuel, J.C., \& Avis, N.E. (2005). The impact of breast cancer on younger women's relationships with their partner and children. Families, Systems, \& Health, 23(1), 80-93. http://dx.doi.org/10.1037/1091-7527.23.1.80.

Webster, K., Cella, D., Yost, K. (2003). The Functional Assessment of Chronic Illness Therapy (FACIT) Measurement System: Properties, applications, and interpretation. Health \&Quality of Life Outcomes, 1, 79. doi:10.1186/1477-7525-179. 
Webster, K., Odom, L., Peterman, A., Lent, L., \& Cella, D. (1999). The Functional Assessment of Chronic Illness Therapy (FACIT) measurement system: Validation of version 4 of the core questionnaire. Quality of Life Research, 8(7), 604. doi: $10.1186 / 1477-7525-1-79$.

Weihs, K., Fisher, L., \& Baird, M. . (2002). Families and the management of chronic disease: Report for the Committee on Health and Behavior. Research Practice and Policy; Institute of Medicine, National Academy of Sciences. Families, Systems and Health, 20(1), 7-46. doi: 10.1037/h0089481.

Weiss, T. (2002). Posttraumatic growth in women with breast cancer and their husbands: An intersubjective validation study. Journal of Psychosocial Oncology, 20(2), 6580. doi:10.1300/j077v20n02_04.

Weiss, T. (2004). Correlates of posttraumatic growth in husbands of breast cancer survivors. Psycho-Oncology, 13(4), 260-268. doi: 10.1002/pon.735.

Wen, K.Y., Fang, C.Y., \& Ma, G.X. (2014). Breast cancer experience and survivorship among Asian Americans: A systematic review. Journal of Cancer Survivorship, 8(1):94-107. doi: 10.1007/s11764-013-0320-8.

Wenzel, L., Dogan-Ates, A., Habbal, R., Berkowitz, R., Goldstein, D.P., Bernstein,M., Kluhsman, B.C., Osann, K., Newlands, E., Seckl,M.J., Hancock, B. \& and Cella, D. (2005). Defining and measuring reproductive concerns of female cancer survivors. Journal of the National Cancer Institute. Monographs, 34, 94-98. doi: 10.1093/jncimonographs/lgi017. 
Wenzel, L., Glanz, K., \& Lerman, C. (2002). Stress, coping, and health behavior. In B. K. R. K. Glanz, \& F.M. Lewis (Ed.), Health behavior and health education: Theory, research, and practice. San Francisco: CA: Jossey-Bass.

Wenzel, L.B., Fairclough, D.L., Brady, M.J., Cella, D., Garrett, K.M., Klushman, B.C., Crane, L.A.,\& Marcus, A. (1999). Age-related differences in the quality of life of breast carcinoma patients after treatment. Cancer, 86(9), 1768-1774. doi:

10.1002/(sici)1097-0142(19991101)86:9<1768::aid-cncr19>3.0.co;2-o.

Whitchurch, G. G. \& Constantine, L.L. (1993). Systems Theory. In P. G. Boss, Doherty, W.J., LaRossa, R., Schumm, W.R., \& Steinmetz, S.K. (Ed.), Sourcebook of Family Theories and Methods. A Contextual Approach (pp. 325-352). New York, NY: Plenum Press.

White, J.M. (1991). Dynamics of Family Development: A theoretical perspective. New York, NY: Guildford.

White, J. M., \& Klein, D. M. (2002). Family Theories (2nd Edition). Thousand Oaks, CA: Sage Publications, Inc.

White, J. M., \& Klein, D. M. (2008). Family Theories (3rd Edition). Thousand Oaks, CA: Sage.

White, J. M., Klein, D. M. \& Martin, T.F.(2015). Family Theories (4nd Edition). Thousand Oaks, CA: Sage Publications, Inc.

Williams, A.L., \& McCorkle, R. (2011). Cancer family caregivers during the palliative, hospice, and bereavement phases: a review of the descriptive psychosocial literature. Palliative \& Supportive Care, 9(3):315-325. doi:

10.1017/S1478951511000265. 
Williamson,H.C., Karney, B.R., \& Bradbury, T.N.(2013). Financial strain and stressful events predict newlyweds' negative communication independent of relationship satisfaction. Journal of Family Psychology, 27(1), 65-67. doi: 10.1037/a003110465.

Wimberly, S.R., Carver, C.S., Laurenceau, J.P., Harris, S.D., \& Antoni, M.H.(2005). Perceived partner reactions to diagnosis and treatment of breast cancer: impact on psychosocial and psychosexual adjustment. Journal of Consulting Clinical Psychology, 73(2), 300-11.doi: 10.1037/0022-006x.73.2.300.

Winnicott, D. W. (1960). The Theory of Parent-Infant Relationships. The Maturation Process and the Facilitating Environment. New York: International Universities Press.

Winstead-Fry, P., \& Schultz, A. (1997). Psychometric assessment of the Functional Assessment of Cancer Therapy-General (FACT-G) scale in a rural sample. Cancer, 79(12), 2446-2452. doi: 10.1002/(sici)1097-0142(19970615)79:12<2446::aidcncr23>3.0.co;2-q.

Wittenborn, A. K., Dolbin-MacNab, M. L., \& Keiley, M. K. (2013). Dyadic research in marriage and family therapy: Methodological considerations. Journal of Marital and Family Therapy, 39(1), 5-16. doi: 10.1111/j.1752-0606.2012.00306.x.

Wittmann, D., Skolarus, T.A., \& Monti, J.E. (2015). Are we targeting the right outcome for sexual health after prostate cancer treatment? European Urology, 68(4), 550551. doi: 10.1016/j.eururo.2015.05.044.

Woloski-Wruble, A. C., Ganz, F.D., Jiang, Y., Qiang, W.M., \& Kadmon, I. (2012). Israeli and Chinese partners of women with breast cancer: A cross-cultural view of marital issues. Psycho-Oncology, 21(3), 324-331. doi: 10.1002/pon.1899. 
Wong-Kim, E.C., \& Bloom, J.R. (2005). Depression experienced by young women newly diagnosed with breast cancer. Psycho-Oncology, 14(7),564-573. doi: 10.1002/pon.873

World Health Organization (1993). Study protocol for the World Health Organization project to develop a quality of life assessment instrument (WHOQOL). Quality of Life Research, 2(2), 153-159.

Woszidlo, A., \& Segrin, C. (2013). Negative affectivity and educational attainment as predictors of newlyweds' problem solving communication and marital quality. Journal of Psychology, 147(1), 49-73. doi: 10.1080/00223980.2012.674069.

Wunderer, E., \& Schneewind, K. A. (2008). The relationship between marital standards, dyadic coping and marital satisfaction. European Journal of Social Psychology, 38, 462-476. doi: 10.1002/ejsp.405.

Würz, J., Brähler, E., Kapellen, T., Galler, A., Kiess, W. (2006). Dyadisches Coping bei Eltern von Kindern und Jugendlichen mit Diabetes mellitus Typ 1. Kinder und Jugendmedizin, 2, 81-92.

Wyatt, G., Beckrow, K.C., Gardiner, J, \& Pathak, D.(2008). Predictors of postsurgical subacute emotional and physical well-being among women with breast cancer. Cancer Nursing, 31(2):E28-E39. doi: 10.1097/01.NCC.0000305705.91787.55.

Yanez, B., Thompson, E.H., \& Stanton, A.L. (2011). Quality of life among Latina breast cancer patients: A systematic review of the literature. Journal of Cancer Survivorship, 5(2), 191-207. doi: 10.1007/s11764-011-0171-0. 
Yoo, G.J., Levine, E.G., \& Pasick, R. (2014). Breast cancer and coping among women of color: A systematic review of the literature. Supportive Care in Cancer, 22(3), 811824. doi: 10.1007/s00520-013-2057-3.

Yoshimoto, S. M., Ghorbani, S., Baer, J.M., Cheng, K.W., Banthia, R., Malcarne, V.L., \& Varni, J.W. (2006). Religious coping and problem-solving by couples faced with prostate cancer. European Journal of Cancer Care, 15(5), 481-488. doi:

10.1111/j.1365-2354.2006.00700.x.

Yu, Y., \& Sherman, K.A. (2015). Communication avoidance, coping and psychological distress of women with breast cancer. Journal of Behavioral Medicine, 38(3), 565577. doi: 10.1007/s10865-015-9636-3.

Yun, Y.H., Kim, S.H., Lee. K.M., Park, S.M., \& Kim, Y.M. (2007). Age, sex and comorbidities were considered in comparing reference data for health-related quality of life in the general and cancer populations. Journal of Clinical Epistemology, 60(11), 1164-1175. doi: http://dx.doi.org/10.1016/j.jclinepi.2006.12.014.

Zafar, S. Y., Abernethy, A. P. (2013). Financial toxicity, part I: A new name for a growing problem. Cancer Network, 27(2), 80-81.

Zafar, S. Y., Peppercorn, J. M., Schrag, D., Taylor, D. H., Goetzinger, A. M., Zhong, X., \& Abernethy, A. P. (2013). The financial toxicity of cancer treatment: A pilot study assessing out-of-pocket expenses and the insured cancer patient's experience. The Oncologist, 18(1), 381-390. doi: 10.1634/theoncologist.2012-0279.

Zani, B., \& Cicognani, E. (2002). Le vie del benessere. Eventi di vita e strategie di coping. Roma: Carocci Editore. 
Zebrack., B.J., Mills, J., \& Weitzman, T.S. (2007). Health and supportive care needs of young adult cancer patients and survivors. Journal of Cancer Survivorship, 1(2), 173-145. doi: 10.1007/s11764-007-0015-0.

Zenger, M., Lehmann-Laue, A., Stolzenburg, J. U., Schwalenberg, T., Ried, A., \& Hinz, A. (2010). The relationship of quality of life and distress in prostate cancer patients compared to the general population. Psychosocial Medicine, 30.doi:10.3205/psm000064.

Zhao, X., Lynch, J.G., \& Chen, Q. (2010). Reconsidering Baron and Kenny: Myths and truths about mediation analysis. Journal of Consumer Research, 37(2), 197-206. . doi: $10.1086 / 651257$.

Zimmermann, T. (2015). Intimate relationships affected by breast cancer: Interventions for couples. Breast Care, 10(2),102-108. doi: 10.1159/000381966.

Zimmermann, T., Heinrichs, N., \& Baucom, D.H.(2007). "Does one size fit all?" moderators in psychosocial interventions for breast cancer patients: A metaanalysis. Annals of Behavioral Medicine, 34(3), 225-239.

Zimmermann, T., Heinrichs, N., \& Scott, J. (2006). CanCOPE «Schritt für Schritt» : Die Effektivität eines partnerschaftlichen Unterstützungsprogramms bei Frauen mit Brust- oder gynäkologischen Krebserkrankungen. Verhaltenstherapie, 16(4), 247255. doi: 10.1159/000096122.

Zimmermann, T., Scott, J. L., \& Heinrichs, N. (2010). Individual and dyadic predictors of body image in women with breast cancer. Psycho-Oncology, 19(10), 1061-1068. doi: 10.1002/pon.1660 
Zwahlen, D., Hagenbuch, N., Carley, M. I., Jenewein, J., \& Buchi, S. (2010).

Posttraumatic growth in cancer patients and partners: Effects of role, gender and the dyad on couples' posttraumatic growth experience. Psycho-oncology, 19(1), 12-20. doi: 10.1002/pon.1486. 


\section{APPENDIX A}

\section{SURVEY - PATIENT}

\section{Coping with Breast Cancer: Patient Questionnaire}

This questionnaire is about your experience with breast cancer. It will help us to assess how you and your partner are dealing with the illness and treatment. All answers are completely confidential.

\section{PART 1. WELL-BEING}

Below is a list of statements that other people with your illness have said are important. Please circle or mark one number per line to indicate your response as it applies to the past 7 days.

\section{PHYSICAL WELL-BEING}

\begin{tabular}{|c|c|c|c|c|c|c|}
\hline & & $\begin{array}{l}\text { Not } \\
\text { at all }\end{array}$ & $\begin{array}{c}\text { A little } \\
\text { bit }\end{array}$ & Somewhat & $\begin{array}{l}\text { Quite } \\
\text { a bit }\end{array}$ & $\begin{array}{l}\text { Very } \\
\text { much }\end{array}$ \\
\hline 1 & I have a lack of energy & 0 & 1 & 2 & 3 & 4 \\
\hline 2 & I have nausea & 0 & 1 & 2 & 3 & 4 \\
\hline 3 & $\begin{array}{l}\text { Because of my physical } \\
\text { condition, I have trouble meeting } \\
\text { the needs of my family }\end{array}$ & 0 & 1 & 2 & 3 & 4 \\
\hline 4 & I have pain & 0 & 1 & 2 & 3 & 4 \\
\hline 5 & $\begin{array}{l}\text { I am bothered by side effects of } \\
\text { treatment }\end{array}$ & 0 & 1 & 2 & 3 & 4 \\
\hline 6 & I feel ill & 0 & 1 & 2 & 3 & 4 \\
\hline 7 & I am forced to spend time in bed & 0 & 1 & 2 & 3 & 4 \\
\hline
\end{tabular}




\begin{tabular}{|c|c|c|c|c|c|c|}
\hline & & $\begin{array}{l}\text { Not at } \\
\text { all }\end{array}$ & $\begin{array}{l}\text { A little } \\
\text { bit }\end{array}$ & Somewhat & $\begin{array}{l}\text { Quite } \\
\text { a bit }\end{array}$ & $\begin{array}{l}\text { Very } \\
\text { much }\end{array}$ \\
\hline 8 & I feel close to my friends & 0 & 1 & 2 & 3 & 4 \\
\hline 9 & $\begin{array}{l}\text { I get emotional support from my } \\
\text { family }\end{array}$ & 0 & 1 & 2 & 3 & 4 \\
\hline 10 & I get support from my friends & 0 & 1 & 2 & 3 & 4 \\
\hline 11 & $\begin{array}{l}\text { My family has accepted my } \\
\text { illness }\end{array}$ & 0 & 1 & 2 & 3 & 4 \\
\hline 12 & $\begin{array}{l}\text { I am satisfied with family } \\
\text { communication about my illness. }\end{array}$ & 0 & 1 & 2 & 3 & 4 \\
\hline 13 & $\begin{array}{l}\text { I feel close to my partner (or the } \\
\text { person who is my main } \\
\text { support) } \\
\text { Regardless of your current level } \\
\text { of sexual activity, please answer } \\
\text { the following question. If you } \\
\text { prefer not to answer it, please } \\
\text { mark this box and go to the next } \\
\text { section. }\end{array}$ & 0 & 1 & 2 & 3 & 4 \\
\hline 14 & I am satisfied with my sex life & 0 & 1 & 2 & 3 & 4 \\
\hline
\end{tabular}

\section{EMOTIONAL WELL-BEING}

\begin{tabular}{|c|c|c|c|c|c|c|}
\hline & & $\begin{array}{l}\text { Not at } \\
\text { all }\end{array}$ & $\begin{array}{c}A \\
\text { little } \\
\text { bit }\end{array}$ & Somewhat & $\begin{array}{l}\text { Quite } \\
\text { a bit }\end{array}$ & $\begin{array}{l}\text { Very } \\
\text { much }\end{array}$ \\
\hline 15 & I feel sad & 0 & 1 & 2 & 3 & 4 \\
\hline 16 & $\begin{array}{l}\text { I am satisfied with how I am } \\
\text { coping with my illness }\end{array}$ & 0 & 1 & 2 & 3 & 4 \\
\hline 17 & $\begin{array}{l}\text { I am losing hope in the fight } \\
\text { against my illness }\end{array}$ & 0 & 1 & 2 & 3 & 4 \\
\hline 18 & I feel nervous & 0 & 1 & 2 & 3 & 4 \\
\hline 19 & I worry about dying & 0 & 1 & 2 & 3 & 4 \\
\hline 20 & $\begin{array}{l}\text { I worry that my condition will } \\
\text { get worse }\end{array}$ & 0 & 1 & 2 & 3 & 4 \\
\hline
\end{tabular}

\section{FUNCTIONAL WELL-BEING}

\begin{tabular}{|c|c|c|c|c|c|c|}
\hline & & $\begin{array}{c}\text { Not at } \\
\text { all }\end{array}$ & $\begin{array}{c}\text { A } \\
\text { little } \\
\text { bit }\end{array}$ & Somewhat & $\begin{array}{c}\text { Quite } \\
\text { a bit }\end{array}$ & $\begin{array}{l}\text { Very } \\
\text { much }\end{array}$ \\
\hline 21 & $\begin{array}{l}\text { I am able to work (include work } \\
\text { at home) }\end{array}$ & 0 & 1 & 2 & 3 & 4 \\
\hline 22 & $\begin{array}{l}\text { My work (include work at home) } \\
\text { is fulfilling }\end{array}$ & 0 & 1 & 2 & 3 & 4 \\
\hline 23 & I am able to enjoy life & 0 & 1 & 2 & 3 & 4 \\
\hline 24 & I have accepted my illness & 0 & 1 & 2 & 3 & 4 \\
\hline 25 & I am sleeping well & 0 & 1 & 2 & 3 & 4 \\
\hline
\end{tabular}




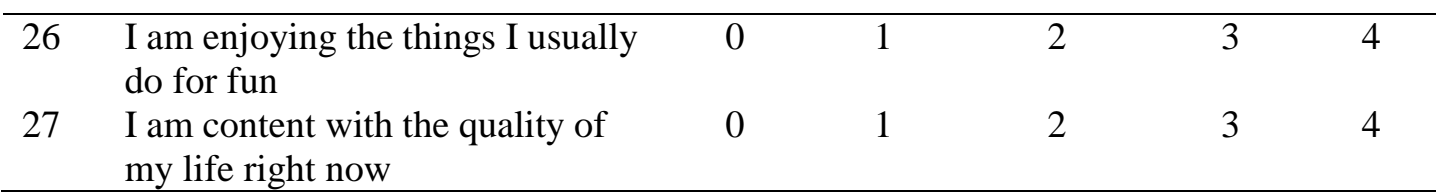

\section{ADDITIONAL CONCERNS}

\begin{tabular}{|c|c|c|c|c|c|c|}
\hline & & $\begin{array}{l}\text { Not at } \\
\text { all }\end{array}$ & $\begin{array}{c}\text { little } \\
\text { bit }\end{array}$ & Somewhat & $\begin{array}{l}\text { Quite } \\
\text { a bit }\end{array}$ & $\begin{array}{l}\text { Very } \\
\text { much }\end{array}$ \\
\hline 28 & I have been short of breath & 0 & 1 & 2 & 3 & 4 \\
\hline 29 & $\begin{array}{l}\text { I am self-conscious about the } \\
\text { way I dress }\end{array}$ & 0 & 1 & 2 & 3 & 4 \\
\hline 30 & $\begin{array}{l}\text { One or both of my arms are } \\
\text { swollen or tender }\end{array}$ & 0 & 1 & 2 & 3 & 4 \\
\hline 31 & I feel sexually attractive & 0 & 1 & 2 & 3 & 4 \\
\hline 32 & I am bothered by hair loss & 0 & 1 & 2 & 3 & 4 \\
\hline 33 & $\begin{array}{l}\text { I worry that other members of } \\
\text { my family might } \\
\text { someday get the same illness I } \\
\text { have }\end{array}$ & 0 & 1 & 2 & 3 & 4 \\
\hline 34 & $\begin{array}{l}\text { I worry about the effect of stress } \\
\text { on my illness }\end{array}$ & 0 & 1 & 2 & 3 & 4 \\
\hline 35 & $\begin{array}{l}\text { I am bothered by a change in } \\
\text { weight }\end{array}$ & 0 & 1 & 2 & 3 & 4 \\
\hline 36 & I am able to feel like a woman & 0 & 1 & 2 & 3 & 4 \\
\hline 37 & $\begin{array}{l}\text { I have certain parts of my body } \\
\text { where I experience pain }\end{array}$ & 0 & 1 & 2 & 3 & 4 \\
\hline
\end{tabular}

PART II. PERSONAL RELATIONSHIPS

We would like you to tell us about your relationship with your spouse or partner. By partner we mean a person with whom you live or with whom you have a steady relationship.

If married, how many years?

What is your spouse's age?

If not married, how long have you known your partner?

What is your partner's age?

Are you currently living with your partner?

YES

NO 


\section{MUTUALITY}

In this section we would like to explore certain aspects of your relationship with your spouse or partner. Using the scale below, please circle the appropriate number to tell us your best estimate of how often you and your spouse./partner experience each of the following.

When we talk about things that matter to my spouse/partner, I am likely to...

\begin{tabular}{lcccccc}
\hline & Never & Rarely & Occasionally & $\begin{array}{c}\text { More } \\
\text { often } \\
\text { than not }\end{array}$ & $\begin{array}{c}\text { Most of } \\
\text { the time }\end{array}$ & $\begin{array}{c}\text { All } \\
\text { the } \\
\text { time }\end{array}$ \\
\hline Be receptive & 1 & 2 & 3 & 4 & 5 & 6 \\
Get impatient & 1 & 2 & 3 & 4 & 5 & 6 \\
Try to understand & 1 & 2 & 3 & 4 & 5 & 6 \\
$\begin{array}{l}\text { Get bored } \\
\text { Feel moved }\end{array}$ & 1 & 2 & 3 & 4 & 5 & 6 \\
$\begin{array}{l}\text { Avoid being honest } \\
\text { Be open-minded }\end{array}$ & 1 & 2 & 3 & 4 & 5 & 6 \\
$\begin{array}{l}\text { Get discouraged } \\
\text { Get involved }\end{array}$ & 1 & 2 & 3 & 4 & 5 & 6 \\
$\begin{array}{l}\text { Have difficulty } \\
\text { listening }\end{array}$ & 1 & 2 & 3 & 4 & 5 & 6 \\
$\begin{array}{l}\text { Feel energized by our } \\
\text { conversation }\end{array}$ & 1 & 2 & 3 & 4 & 5 & 6 \\
\hline
\end{tabular}

When we talk about things that matter to me, my spouse/partner is likely to...

\begin{tabular}{|c|c|c|c|c|c|c|}
\hline & Never & Rarely & Occasionally & $\begin{array}{l}\text { More } \\
\text { than } \\
\text { often }\end{array}$ & $\begin{array}{l}\text { Most of } \\
\text { the time }\end{array}$ & $\begin{array}{c}\text { All } \\
\text { the } \\
\text { time }\end{array}$ \\
\hline Pick up on my feelings & 1 & 2 & 3 & 4 & 5 & 6 \\
\hline $\begin{array}{l}\text { Feel like we are not } \\
\text { getting anywhere }\end{array}$ & 1 & 2 & 3 & 4 & 5 & 6 \\
\hline Show an interest & 1 & 2 & 3 & 4 & 5 & 6 \\
\hline Get frustrated & 1 & 2 & 3 & 4 & 5 & 6 \\
\hline $\begin{array}{l}\text { Share similar } \\
\text { experiences }\end{array}$ & 1 & 2 & 3 & 4 & 5 & 6 \\
\hline Keep feelings inside & 1 & 2 & 3 & 4 & 5 & 6 \\
\hline $\begin{array}{l}\text { Respect my point of } \\
\text { view }\end{array}$ & 1 & 2 & 3 & 4 & 5 & 6 \\
\hline Change the subject & 1 & 2 & 3 & 4 & 5 & 6 \\
\hline $\begin{array}{l}\text { See the humor in } \\
\text { things }\end{array}$ & 1 & 2 & 3 & 4 & 5 & 6 \\
\hline Feel down & 1 & 2 & 3 & 4 & 5 & 6 \\
\hline $\begin{array}{l}\text { Express an opinion } \\
\text { clearly }\end{array}$ & 1 & 2 & 3 & 4 & 5 & 6 \\
\hline
\end{tabular}




\section{PART III. COPING}

\section{Section 1}

This section is about what you do when you are felling stressed. Please respond by circling the appropriate number.

\begin{tabular}{|c|c|c|c|c|c|c|c|}
\hline & & Never & Rarely & Sometimes & Often & $\begin{array}{c}\text { Very } \\
\text { Often } \\
\end{array}$ & Always \\
\hline 1 & $\begin{array}{l}\text { I let my partner know that I } \\
\text { appreciate his/her support, } \\
\text { advice, or help }\end{array}$ & 1 & 2 & 3 & 4 & 5 & 6 \\
\hline 2 & $\begin{array}{l}\text { I ask my partner to do things } \\
\text { for me when I have too } \\
\text { much to do }\end{array}$ & 1 & 2 & 3 & 4 & 5 & 6 \\
\hline 3 & $\begin{array}{l}\text { I show my partner through } \\
\text { my behavior that I am not } \\
\text { doing well or that I have } \\
\text { problems }\end{array}$ & 1 & 2 & 3 & 4 & 5 & 6 \\
\hline 4 & $\begin{array}{l}\text { I tell my partner openly how } \\
\text { I feel and that I would } \\
\text { appreciate his/her support }\end{array}$ & 1 & 2 & 3 & 4 & 5 & 6 \\
\hline 5 & $\begin{array}{l}\text { I try to hide my stress from } \\
\text { my partner so that she/he } \\
\text { does not notice it }\end{array}$ & 1 & 2 & 3 & 4 & 5 & 6 \\
\hline
\end{tabular}

\section{Section 2}

This section is about what your partner does when you are feeling stressed

\begin{tabular}{|c|c|c|c|c|c|c|c|}
\hline & & Never & Rarely & Sometimes & Often & $\begin{array}{l}\text { Very } \\
\text { Often }\end{array}$ & Always \\
\hline 6 & $\begin{array}{l}\text { My partner gives me feeling } \\
\text { that he/she understands me }\end{array}$ & 1 & 2 & 3 & 4 & 5 & 6 \\
\hline 7 & $\begin{array}{l}\text { My partner expresses that } \\
\text { he/she is no my side }\end{array}$ & 1 & 2 & 3 & 4 & 5 & 6 \\
\hline 8 & $\begin{array}{l}\text { My partner makes fun of me } \\
\text { when I am feeling stressed }\end{array}$ & 1 & 2 & 3 & 4 & 5 & 6 \\
\hline 9 & $\begin{array}{l}\text { My partner helps me see the } \\
\text { stressful situation in a } \\
\text { different light }\end{array}$ & 1 & 2 & 3 & 4 & 5 & 6 \\
\hline 10 & $\begin{array}{l}\text { My partner listens to me and } \\
\text { gives me the opportunity to } \\
\text { communicate the entire } \\
\text { situation }\end{array}$ & 1 & 2 & 3 & 4 & 5 & 6 \\
\hline 11 & $\begin{array}{l}\text { My partner lets me know } \\
\text { that he/she does not want to } \\
\text { be bothered with my } \\
\text { problems }\end{array}$ & 1 & 2 & 3 & 4 & 5 & 6 \\
\hline 12 & $\begin{array}{l}\text { My partner takes me in } \\
\text { his/her arms and is tender } \\
\text { toward me }\end{array}$ & 1 & 2 & 3 & 4 & 5 & 6 \\
\hline
\end{tabular}




\begin{tabular}{|c|c|c|c|c|c|c|c|}
\hline 13 & $\begin{array}{l}\text { My partner consoles me to } \\
\text { make me feel better }\end{array}$ & 1 & 2 & 3 & 4 & 5 & 6 \\
\hline 14 & $\begin{array}{l}\text { My partner tells me that my } \\
\text { way of stress bothers } \\
\text { him/her }\end{array}$ & 1 & 2 & 3 & 4 & 5 & 6 \\
\hline 15 & $\begin{array}{l}\text { My partner takes on things } \\
\text { that I normally do in order } \\
\text { to help me out }\end{array}$ & 1 & 2 & 3 & 4 & 5 & 6 \\
\hline 16 & $\begin{array}{l}\text { Although my partner makes } \\
\text { time for me, his/her } \\
\text { thoughts are somewhere else }\end{array}$ & 1 & 2 & 3 & 4 & 5 & 6 \\
\hline 17 & $\begin{array}{l}\text { My partner shows me that } \\
\text { he/she is standing by me }\end{array}$ & 1 & 2 & 3 & 4 & 5 & 6 \\
\hline 18 & $\begin{array}{l}\text { When I am too busy my } \\
\text { partner helps me out }\end{array}$ & 1 & 2 & 3 & 4 & 5 & 6 \\
\hline 19 & $\begin{array}{l}\text { My partner praises me when } \\
\text { he/she realizes I have } \\
\text { handled stress well }\end{array}$ & 1 & 2 & 3 & 4 & 5 & 6 \\
\hline 20 & $\begin{array}{l}\text { My partner finds it okay } \\
\text { when I want to be alone }\end{array}$ & 1 & 2 & 3 & 4 & 5 & 6 \\
\hline 21 & $\begin{array}{l}\text { My partner helps me to } \\
\text { think through the situation } \\
\text { so that I can understand } \\
\text { better what is going on. }\end{array}$ & 1 & 2 & 3 & 4 & 5 & 6 \\
\hline 22 & $\begin{array}{l}\text { My partner helps me out and } \\
\text { takes on tasks when I ask } \\
\text { him /her to do so }\end{array}$ & 1 & 2 & 3 & 4 & 5 & 6 \\
\hline 23 & $\begin{array}{l}\text { My partner helps me to } \\
\text { relax by giving me a } \\
\text { massage or something like } \\
\text { that }\end{array}$ & 1 & 2 & 3 & 4 & 5 & 6 \\
\hline 24 & $\begin{array}{l}\text { I am satisfied with the } \\
\text { support I receive from my } \\
\text { partner }\end{array}$ & 1 & 2 & 3 & 4 & 5 & 6 \\
\hline 25 & $\begin{array}{l}\text { I found the support that I } \\
\text { receive from my partner is } \\
\text { helpful and effective }\end{array}$ & 1 & 2 & 3 & 4 & 5 & 6 \\
\hline
\end{tabular}

\section{Section 3}

This section is about what you do when your partner is feeling stressed

\begin{tabular}{|c|c|c|c|c|c|c|c|}
\hline & & Never & Rarely & Sometimes & Often & $\begin{array}{l}\text { Very } \\
\text { Often }\end{array}$ & Always \\
\hline 26 & $\begin{array}{l}\text { I communicate } \\
\text { understanding to my parent }\end{array}$ & 1 & 2 & 3 & 4 & 5 & 6 \\
\hline 27 & $\begin{array}{l}\text { I express to my partner that } \\
\text { I am on his/her side }\end{array}$ & 1 & 2 & 3 & 4 & 5 & 6 \\
\hline 28 & $\begin{array}{l}\text { I make fun of my partner's } \\
\text { stress }\end{array}$ & 1 & 2 & 3 & 4 & 5 & 6 \\
\hline 29 & $\begin{array}{l}\text { I tell my partner that his/her } \\
\text { stress is not that bad and }\end{array}$ & 1 & 2 & 3 & 4 & 5 & 6 \\
\hline
\end{tabular}




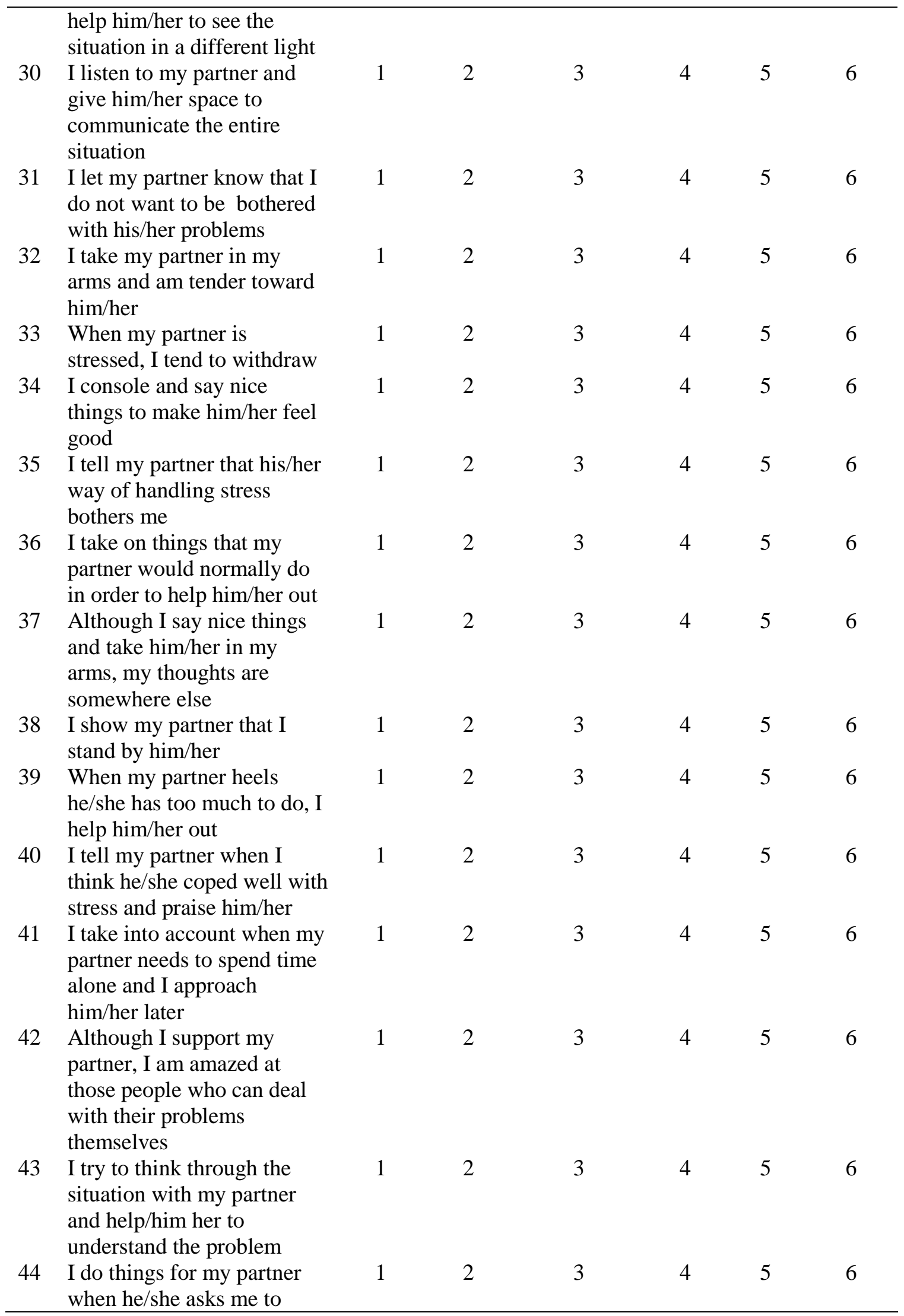




\begin{tabular}{|c|c|c|c|c|c|c|c|}
\hline 45 & $\begin{array}{l}\text { I help my partner to relax by } \\
\text { giving him/her a massage } \\
\text { or something like that }\end{array}$ & 1 & 2 & 3 & 4 & 5 & 6 \\
\hline 46 & $\begin{array}{l}\text { When my partner is } \\
\text { stressed, I tend to get out of } \\
\text { his/her way }\end{array}$ & 1 & 2 & 3 & 4 & 5 & 6 \\
\hline 47 & $\begin{array}{l}\text { My partner is satisfied with } \\
\text { the support I give to him/her }\end{array}$ & 1 & 2 & 3 & 4 & 5 & 6 \\
\hline 48 & $\begin{array}{l}\text { The support I give to my } \\
\text { partner is helpful and } \\
\text { effective }\end{array}$ & 1 & 2 & 3 & 4 & 5 & 6 \\
\hline
\end{tabular}

\section{Section 4}

This section is about what you and your partner do when you are both feeling stressed

\begin{tabular}{|c|c|c|c|c|c|c|c|}
\hline & & Never & Rarely & Sometimes & Often & $\begin{array}{l}\text { Very } \\
\text { Often }\end{array}$ & Always \\
\hline 49 & $\begin{array}{l}\text { We are supportive of each } \\
\text { other and help one another } \\
\text { out }\end{array}$ & 1 & 2 & 3 & 4 & 5 & 6 \\
\hline 50 & $\begin{array}{l}\text { We divide tasks fairly so } \\
\text { that both of us have an } \\
\text { equal amount of work to do }\end{array}$ & 1 & 2 & 3 & 4 & 5 & 6 \\
\hline 51 & $\begin{array}{l}\text { As a couple, we generally } \\
\text { solve our problems on our } \\
\text { own }\end{array}$ & 1 & 2 & 3 & 4 & 5 & 6 \\
\hline 52 & $\begin{array}{l}\text { We have a good laugh } \\
\text { together about it and take it } \\
\text { lightly }\end{array}$ & 1 & 2 & 3 & 4 & 5 & 6 \\
\hline 53 & $\begin{array}{l}\text { We engage in a serious } \\
\text { discussion about the } \\
\text { problem and think through } \\
\text { what has to be done }\end{array}$ & 1 & 2 & 3 & 4 & 5 & 6 \\
\hline 54 & $\begin{array}{l}\text { We meditate or pray } \\
\text { together }\end{array}$ & 1 & 2 & 3 & 4 & 5 & 6 \\
\hline 55 & $\begin{array}{l}\text { We help one another to put } \\
\text { the problem in perspective } \\
\text { and see it in a new light }\end{array}$ & 1 & 2 & 3 & 4 & 5 & 6 \\
\hline 56 & $\begin{array}{l}\text { We help each other relax } \\
\text { with such things like } \\
\text { massage, taking a bath } \\
\text { together, or listening to } \\
\text { music }\end{array}$ & 1 & 2 & 3 & 4 & 5 & 6 \\
\hline 57 & $\begin{array}{l}\text { We sit down to talk } \\
\text { together and share our } \\
\text { feelings }\end{array}$ & 1 & 2 & 3 & 4 & 5 & 6 \\
\hline 58 & $\begin{array}{l}\text { When we are both stressed, } \\
\text { we withdraw and avoid } \\
\text { each other }\end{array}$ & 1 & 2 & 3 & 4 & 5 & 6 \\
\hline 59 & $\begin{array}{l}\text { We caress one another and } \\
\text { make love }\end{array}$ & 1 & 2 & 3 & 4 & 5 & 6 \\
\hline
\end{tabular}




\begin{tabular}{|c|c|c|c|c|c|c|c|}
\hline 60 & $\begin{array}{l}\text { I am satisfied with the way } \\
\text { my partner and I deal with } \\
\text { stress together }\end{array}$ & 1 & 2 & 3 & 4 & 5 & 6 \\
\hline 61 & $\begin{array}{l}\text { I found as a couple, the } \\
\text { way we deal with stress } \\
\text { together is effective }\end{array}$ & 1 & 2 & 3 & 4 & 5 & 6 \\
\hline
\end{tabular}

\section{PART IV. COMMUNICATION}

Next to each statement below, circle the number of the response that best describes the way you feel about the statement

\begin{tabular}{|c|c|c|c|c|c|c|}
\hline & & $\begin{array}{l}\text { Definitely } \\
\text { False }\end{array}$ & $\begin{array}{l}\text { Usually } \\
\text { False }\end{array}$ & $\begin{array}{l}\text { Neither } \\
\text { True or } \\
\text { False }\end{array}$ & $\begin{array}{l}\text { Usually } \\
\text { True }\end{array}$ & $\begin{array}{l}\text { Definitely } \\
\text { True }\end{array}$ \\
\hline 1 & $\begin{array}{l}\text { It is very easy for me to } \\
\text { express all my true } \\
\text { feelings to my partner }\end{array}$ & 1 & 2 & 3 & 4 & 5 \\
\hline 2 & $\begin{array}{l}\text { When we are having a } \\
\text { problem, my partner } \\
\text { often gives me the silent } \\
\text { treatment }\end{array}$ & 1 & 2 & 3 & 4 & 5 \\
\hline 3 & $\begin{array}{l}\text { My partner sometimes } \\
\text { makes comments which } \\
\text { put me down }\end{array}$ & 1 & 2 & 3 & 4 & 5 \\
\hline 4 & $\begin{array}{l}\text { I am sometimes afraid to } \\
\text { ask my partner for what } \\
\text { I want }\end{array}$ & 1 & 2 & 3 & 4 & 5 \\
\hline 5 & $\begin{array}{l}\text { I wish my partner were } \\
\text { more willing to share } \\
\text { his/her feelings with me }\end{array}$ & 1 & 2 & 3 & 4 & 5 \\
\hline 6 & $\begin{array}{l}\text { Sometimes I have } \\
\text { trouble believing } \\
\text { everything my partner } \\
\text { tells me }\end{array}$ & 1 & 2 & 3 & 4 & 5 \\
\hline 7 & $\begin{array}{l}\text { I often do not tell my } \\
\text { partner what I am } \\
\text { feeling because he/she } \\
\text { should already know }\end{array}$ & 1 & 2 & 3 & 4 & 5 \\
\hline 8 & $\begin{array}{l}\text { I am very satisfied with } \\
\text { how my partner and I } \\
\text { talk with each other }\end{array}$ & 1 & 2 & 3 & 4 & 5 \\
\hline 9 & $\begin{array}{l}\text { I do not always share } \\
\text { negative feelings I have } \\
\text { about my partner } \\
\text { because I am afraid } \\
\text { he/she will be angry }\end{array}$ & 1 & 2 & 3 & 4 & 5 \\
\hline 10 & $\begin{array}{l}\text { My partner is always a } \\
\text { good listener }\end{array}$ & 1 & 2 & 3 & 4 & 5 \\
\hline
\end{tabular}




\section{BACKGROUND INFORMATION}

Please provide the following information about yourself. Remember that this and all other information will be kept strictly confidential

Age:

Number of Children:

Ethnic Background:

Asian

Caucasian

Native American/Indian

Afro-American

$\square$ Latino

$\square$ Other:

Education:

Less than high school

High school graduate

$\square$ High school plus some college

$\square$ College graduate

$\square$ College plus some graduate hours

$\square$ Master's degree

$\square \quad \mathrm{PhD}$. MD, JD

$\square$ Other:

Occupation:
Unskilled labor
Managerial
$\square$ Homemaker/parent
$\square \quad$ Skilled labor
$\square$ Professional
$\square$ Other:

Income:
Less than $\$ 10,000$
$\$ 10,000$ - \$29,999
$\$ 30,000$ - \$24,999
$\$ 50,000$ - \$69,999
$\$ 70,000$ - $\$ 89,999$
$\$ 90,000+$ 
Religious affiliation:

Catholic

$\square$ Protestant

$\square$ Jewish

$\square$ Atheist/Agnostic

$\square$ Other:

Date of Diagnosis:

Please list any medications that you are currently taking:

\section{Health History:}

Has anyone in your family received treatment for mental illness?

$\square$ Yes

$\square$ No

If yes, how is the person related to you?

Parent

$\square$ Sibling

$\square$ Grandparent

$\square \quad$ Other (please specify):

Have you ever received treatment for depression?

$\square$ Yes

$\square$ No

If yes, was it before or after your cancer diagnosis

$\square$ Before

After

Thank you very much for completing the questionnaire.

Please remember to return your questionnaires in the separate envelopes 


\section{SURVEY - PARTNER}

\section{Coping with Breast Cancer: Partner Questionnaire}

This questionnaire is about your experience with breast cancer. It will help us to assess how you and your partner are dealing with the illness and treatment. All answers are completely confidential.

\section{PART 1. WELL-BEING}

Please indicate how often you have felt concerned or worried by circling the number which indicates how true each statement has been for you during the past 2 weeks.

How often during the past 2 weeks have you...

\begin{tabular}{|c|c|c|c|c|c|c|c|c|}
\hline & & $\begin{array}{l}\text { All of } \\
\text { the } \\
\text { time }\end{array}$ & $\begin{array}{l}\text { Most of } \\
\text { the } \\
\text { time }\end{array}$ & $\begin{array}{l}\text { A good } \\
\text { bit of the } \\
\text { time }\end{array}$ & $\begin{array}{l}\text { Some } \\
\text { of the } \\
\text { time }\end{array}$ & $\begin{array}{l}\text { A } \\
\text { little } \\
\text { of the } \\
\text { time }\end{array}$ & $\begin{array}{l}\text { Hardly } \\
\text { any of } \\
\text { the } \\
\text { time }\end{array}$ & $\begin{array}{l}\text { None } \\
\text { of the } \\
\text { time }\end{array}$ \\
\hline 1 & $\begin{array}{l}\text { Felt concerned or } \\
\text { worried? }\end{array}$ & 1 & 2 & 3 & 4 & 5 & 6 & 7 \\
\hline 2 & $\begin{array}{l}\text { Felt questioning or } \\
\text { uninformed? }\end{array}$ & 1 & 2 & 3 & 4 & 5 & 6 & 7 \\
\hline 3 & Felt tense or upset? & 1 & 2 & 3 & 4 & 5 & 6 & 7 \\
\hline 4 & Felt sad or upset? & 1 & 2 & 3 & 4 & 5 & 6 & 7 \\
\hline 5 & $\begin{array}{l}\text { Felt nervous or } \\
\text { anxious? }\end{array}$ & 1 & 2 & 3 & 4 & 5 & 6 & 7 \\
\hline 6 & Felt overprotective? & 1 & 2 & 3 & 4 & 5 & 6 & 7 \\
\hline 7 & $\begin{array}{l}\text { Had trouble getting a } \\
\text { good night's sleep, or } \\
\text { found yourself } \\
\text { watching your partner } \\
\text { while he is sleeping? }\end{array}$ & 1 & 2 & 3 & 4 & 5 & 6 & 7 \\
\hline 8 & $\begin{array}{l}\text { Felt disappointed or } \\
\text { discouraged? }\end{array}$ & 1 & 2 & 3 & 4 & 5 & 6 & 7 \\
\hline 9 & $\begin{array}{l}\text { Felt frustrated or } \\
\text { angry? }\end{array}$ & 1 & 2 & 3 & 4 & 5 & 6 & 7 \\
\hline 10 & Felt tearful or crying? & 1 & 2 & 3 & 4 & 5 & 6 & 7 \\
\hline 11 & $\begin{array}{l}\text { Felt isolated, alone or } \\
\text { lonely? }\end{array}$ & 1 & 2 & 3 & 4 & 5 & 6 & 7 \\
\hline 12 & $\begin{array}{l}\text { Felt helpless or } \\
\text { insecure? }\end{array}$ & 1 & 2 & 3 & 4 & 5 & 6 & 7 \\
\hline 13 & $\begin{array}{l}\text { Happy, satisfied or } \\
\text { pleased with your } \\
\text { personal life? }\end{array}$ & 1 & 2 & 3 & 4 & 5 & 6 & 7 \\
\hline 14 & $\begin{array}{l}\text { Felt apprehensive or } \\
\text { frightened? }\end{array}$ & 1 & 2 & 3 & 4 & 5 & 6 & 7 \\
\hline
\end{tabular}




\section{IMPACT OF ILLNESS}

The following items ask about how much your partner's illness and/or its treatment interfere with different aspects of your life. Please circle the one number that best describes your current life situation. If an item is not applicable, please circle the number 1 to indicate that this aspect of your life is not affected very much. Please do not leave any item unanswered.

How much does your partner's illness and its treatment interfere with your...

\begin{tabular}{|c|c|c|c|c|c|c|c|c|c|}
\hline 1 & HEALTH & & & & & & & & \\
\hline & Not Very Much & 1 & 2 & 3 & 4 & 5 & 6 & 7 & Very Much \\
\hline 2 & DIET & & & & & & & & Very Much \\
\hline & Not Very Much & 1 & 2 & 3 & 4 & 5 & 6 & 7 & \\
\hline 3 & WORK & & & & & & & & \\
\hline & Not Very Much & 1 & 2 & 3 & 4 & 5 & 6 & 7 & Very Much \\
\hline 4 & ACTIVE & & & & & & & & \\
\hline & RECREATION & 1 & 2 & 3 & 4 & 5 & 6 & 7 & Very Much \\
\hline & Not Very Much & & & & & & & & \\
\hline 5 & PASSIVE & & & & & & & & \\
\hline & RECREATION & & & & & & & & \\
\hline & Not Very Much & 1 & 2 & 3 & 4 & 5 & 6 & 7 & Very Much \\
\hline 6 & FINANCIAL & & & & & & & & \\
\hline & SITUATION & & & & & & & & \\
\hline & Not Very Much & 1 & 2 & 3 & 4 & 5 & 6 & 7 & Very Much \\
\hline 7 & RELATIONSHIP & & & & & & & & \\
\hline & WITH YOUR & & & & & & & & \\
\hline & SPOUSE OR & & & & & & & & \\
\hline & PARTNER & 1 & 2 & 3 & 4 & 5 & 6 & 7 & Very Much \\
\hline & Not Very Much & & & & & & & & \\
\hline 8 & SEX LIFE & & & & & & & & \\
\hline & Not Very Much & 1 & 2 & 3 & 4 & 5 & 6 & 7 & Very Much \\
\hline 9 & FAMILY & & & & & & & & \\
\hline & RELATIONS & 1 & 2 & 3 & 4 & 5 & 6 & 7 & Very Much \\
\hline & Not Very Much & & & & & & & & \\
\hline 10 & OTHER SOCIAL & & & & & & & & \\
\hline & RELATIONS & & & & & & & & \\
\hline & Not Very Much & 1 & 2 & 3 & 4 & 5 & 6 & 7 & Very Much \\
\hline 11 & SELF- & & & & & & & & \\
\hline & EXPRESSION/SELF- & & & & & & & & \\
\hline & IMPROVEMENT & & & & & & & & \\
\hline & Not Very Much & 1 & 2 & 3 & 4 & 5 & 6 & 7 & Very Much \\
\hline 12 & RELIGIOUS & & & & & & & & \\
\hline & EXPRESSION & & & & & & & & \\
\hline & Not Very Much & 1 & 2 & 3 & 4 & 5 & 6 & 7 & Very Much \\
\hline 13 & COMMUNITY & & & & & & & & \\
\hline & INVOLVEMENT & & & & & & & & \\
\hline & Not Very Much & 1 & 2 & 3 & 4 & 5 & 6 & 7 & Very Much \\
\hline
\end{tabular}




\section{PART II. PERSONAL RELATIONSHIPS}

We would like you to tell us about your relationship with your spouse or partner. By partner we mean a person with whom you live or with whom you have a steady relationship.

If married, how many years?

What is your spouse's age?

If not married, how long have you known your partner?

What is your partner's age?

Are you currently living with your partner?

YES

NO

\section{MUTUALITY}

In this section we would like to explore certain aspects of your relationship with your spouse or partner. Using the scale below, please circle the appropriate number to tell us your best estimate of how often you and your spouse/partner experience each of the following.

When we talk about things that matter to my spouse/partner, I am likely to...

\begin{tabular}{lcccccc}
\hline & Never & Rarely & Occasionally & $\begin{array}{l}\text { More } \\
\text { often } \\
\text { than not }\end{array}$ & $\begin{array}{l}\text { Most } \\
\text { of the } \\
\text { time }\end{array}$ & $\begin{array}{l}\text { All } \\
\text { the } \\
\text { time }\end{array}$ \\
\hline Be receptive & 1 & 2 & 3 & 4 & 5 & 6 \\
$\begin{array}{l}\text { Get impatient } \\
\text { Try to understand }\end{array}$ & 1 & 2 & 3 & 4 & 5 & 6 \\
$\begin{array}{l}\text { Get bored } \\
\text { Feel moved }\end{array}$ & 1 & 2 & 3 & 4 & 5 & 6 \\
$\begin{array}{l}\text { Avoid being honest } \\
\text { Be open-minded }\end{array}$ & 1 & 2 & 3 & 4 & 5 & 6 \\
$\begin{array}{l}\text { Get discouraged } \\
\text { Get involved }\end{array}$ & 1 & 2 & 3 & 4 & 5 & 6 \\
$\begin{array}{l}\text { Have difficulty } \\
\text { listening }\end{array}$ & 1 & 2 & 3 & 4 & 5 & 6 \\
$\begin{array}{l}\text { Feel energized by our } \\
\text { conversation }\end{array}$ & 1 & 2 & 3 & 4 & 5 & 6 \\
\hline
\end{tabular}

When we talk about things that matter to me, my spouse/partner is likely to...

\begin{tabular}{|c|c|c|c|c|c|c|}
\hline & Never & Rarely & Occasionally & $\begin{array}{l}\text { More } \\
\text { than } \\
\text { often }\end{array}$ & $\begin{array}{c}\text { Most } \\
\text { of the } \\
\text { time }\end{array}$ & $\begin{array}{c}\text { All } \\
\text { the } \\
\text { time }\end{array}$ \\
\hline Pick up on my feelings & 1 & 2 & 3 & 4 & 5 & 6 \\
\hline $\begin{array}{l}\text { Feel like we are not } \\
\text { getting anywhere }\end{array}$ & 1 & 2 & 3 & 4 & 5 & 6 \\
\hline Show an interest & 1 & 2 & 3 & 4 & 5 & 6 \\
\hline Get frustrated & 1 & 2 & 3 & 4 & 5 & 6 \\
\hline $\begin{array}{l}\text { Share similar } \\
\text { experiences }\end{array}$ & 1 & 2 & 3 & 4 & 5 & 6 \\
\hline Keep feelings inside & 1 & 2 & 3 & 4 & 5 & 6 \\
\hline
\end{tabular}




\begin{tabular}{lllllll}
\hline $\begin{array}{l}\text { Respect my point of } \\
\text { view }\end{array}$ & 1 & 2 & 3 & 4 & 5 & 6 \\
$\begin{array}{l}\text { Change the subject } \\
\text { See the humor in things }\end{array}$ & 1 & 2 & 3 & 4 & 5 & 6 \\
$\begin{array}{l}\text { Feel down } \\
\begin{array}{l}\text { Express an opinion } \\
\text { clearly }\end{array}\end{array}$ & 1 & 2 & 3 & 4 & 5 & 6 \\
\hline
\end{tabular}

\section{PART III. COPING}

\section{Section 1}

This section is about what you do when you are felling stressed. Please respond by circling the appropriate number.

\begin{tabular}{llccccc}
\hline & Never & Rarely & Sometimes & Often & $\begin{array}{c}\text { Very } \\
\text { Often }\end{array}$ & Always \\
\hline $1 \quad \begin{array}{l}\text { I let my partner know } \\
\text { that I appreciate his/her } \\
\text { support, advice, or help }\end{array}$ & 1 & 2 & 3 & 4 & 5 & 6 \\
2 & 1 & 2 & 3 & 4 & 5 & 6 \\
$\begin{array}{l}\text { I ask my partner to do } \\
\text { things for me when I } \\
\text { have too much to do }\end{array}$ & 1 & 2 & 3 & 4 & 5 & 6 \\
$3 \begin{array}{l}\text { I show my partner } \\
\text { through my behavior } \\
\text { that I am not doing well } \\
\text { or that I have problems }\end{array}$ & 1 & 2 & 3 & 4 & 5 & 6 \\
$4 \begin{array}{l}\text { I tell my partner openly } \\
\text { how I feel and that I } \\
\text { would appreciate his/her } \\
\text { support }\end{array}$ & 1 & 2 & 3 & 4 & 5 & 6 \\
$5 \begin{array}{l}\text { I try to hide my stress } \\
\text { from my partner so that } \\
\text { she/he does not notice it }\end{array}$ & 1 & & & & \\
\hline
\end{tabular}

\section{Section 2}

This section is about what your partner does when you are feeling stressed

\begin{tabular}{llccccc}
\hline & Never & Rarely & Sometimes & Often & $\begin{array}{c}\text { Very } \\
\text { Often }\end{array}$ & Always \\
\hline 6 & 1 & 2 & 3 & 4 & 5 & 6 \\
\hline $\begin{array}{l}\text { My partner gives me } \\
\text { feeling that he/she } \\
\text { understands me }\end{array}$ & 1 & 2 & 3 & 4 & 5 & 6 \\
$\begin{array}{l}\text { My partner expresses that } \\
\text { he/she is no my side } \\
\text { My partner makes fun of } \\
\text { me when I am feeling } \\
\text { stressed }\end{array}$ & 1 & 2 & 3 & 4 & 5 & 6 \\
\hline
\end{tabular}




\begin{tabular}{|c|c|c|c|c|c|c|c|}
\hline 9 & $\begin{array}{l}\text { My partner helps me see } \\
\text { the stressful situation in a } \\
\text { different light }\end{array}$ & 1 & 2 & 3 & 4 & 5 & 6 \\
\hline 10 & $\begin{array}{l}\text { My partner listens to me } \\
\text { and gives me the } \\
\text { opportunity to } \\
\text { communicate the entire } \\
\text { situation }\end{array}$ & 1 & 2 & 3 & 4 & 5 & 6 \\
\hline 11 & $\begin{array}{l}\text { My partner lets me know } \\
\text { that he/she does not want } \\
\text { to be bothered with my } \\
\text { problems }\end{array}$ & 1 & 2 & 3 & 4 & 5 & 6 \\
\hline 12 & $\begin{array}{l}\text { My partner takes me in } \\
\text { his/her arms and is tender } \\
\text { toward me }\end{array}$ & 1 & 2 & 3 & 4 & 5 & 6 \\
\hline 13 & $\begin{array}{l}\text { My partner consoles me } \\
\text { to make me feel better }\end{array}$ & 1 & 2 & 3 & 4 & 5 & 6 \\
\hline 14 & $\begin{array}{l}\text { My partner tells me that } \\
\text { my way of stress bothers } \\
\text { him/her }\end{array}$ & 1 & 2 & 3 & 4 & 5 & 6 \\
\hline 15 & $\begin{array}{l}\text { My partner takes on } \\
\text { things that I normally do } \\
\text { in order to help me out }\end{array}$ & 1 & 2 & 3 & 4 & 5 & 6 \\
\hline 16 & $\begin{array}{l}\text { Although my partner } \\
\text { makes time for me, } \\
\text { his/her thoughts are } \\
\text { somewhere else }\end{array}$ & 1 & 2 & 3 & 4 & 5 & 6 \\
\hline 17 & $\begin{array}{l}\text { My partner shows me } \\
\text { that he/she is standing by } \\
\text { me }\end{array}$ & 1 & 2 & 3 & 4 & 5 & 6 \\
\hline 18 & $\begin{array}{l}\text { When I am too busy my } \\
\text { partner helps me out }\end{array}$ & 1 & 2 & 3 & 4 & 5 & 6 \\
\hline 19 & $\begin{array}{l}\text { My partner praises me } \\
\text { when he/she realizes I } \\
\text { have handled stress well }\end{array}$ & 1 & 2 & 3 & 4 & 5 & 6 \\
\hline 20 & $\begin{array}{l}\text { My partner finds it okay } \\
\text { when I want to be alone }\end{array}$ & 1 & 2 & 3 & 4 & 5 & 6 \\
\hline 21 & $\begin{array}{l}\text { My partner helps me to } \\
\text { think through the } \\
\text { situation so that I can } \\
\text { understand better what is } \\
\text { going on. }\end{array}$ & 1 & 2 & 3 & 4 & 5 & 6 \\
\hline 22 & $\begin{array}{l}\text { My partner helps me out } \\
\text { and takes on tasks when I } \\
\text { ask him /her to do so }\end{array}$ & 1 & 2 & 3 & 4 & 5 & 6 \\
\hline 23 & $\begin{array}{l}\text { My partner helps me to } \\
\text { relax by giving me a } \\
\text { massage or something } \\
\text { like that }\end{array}$ & 1 & 2 & 3 & 4 & 5 & 6 \\
\hline
\end{tabular}




\begin{tabular}{|c|c|c|c|c|c|c|c|}
\hline 24 & $\begin{array}{l}\text { I am satisfied with the } \\
\text { support I receive from } \\
\text { my partner }\end{array}$ & 1 & 2 & 3 & 4 & 5 & 6 \\
\hline 25 & $\begin{array}{l}\text { I found the support that I } \\
\text { receive from my partner } \\
\text { is helpful and effective }\end{array}$ & 1 & 2 & 3 & 4 & 5 & 6 \\
\hline
\end{tabular}

\section{Section 3}

This section is about what you do when your partner is feeling stressed

\begin{tabular}{|c|c|c|c|c|c|c|c|}
\hline & & Never & Rarely & Sometimes & Often & $\begin{array}{l}\text { Very } \\
\text { Often }\end{array}$ & Always \\
\hline 26 & $\begin{array}{l}\text { I communicate } \\
\text { understanding to my } \\
\text { parent }\end{array}$ & 1 & 2 & 3 & 4 & 5 & 6 \\
\hline 27 & $\begin{array}{l}\text { I express to my partner } \\
\text { that I am on his/her side }\end{array}$ & 1 & 2 & 3 & 4 & 5 & 6 \\
\hline 28 & $\begin{array}{l}\text { I make fun of my } \\
\text { partner's stress }\end{array}$ & 1 & 2 & 3 & 4 & 5 & 6 \\
\hline 29 & $\begin{array}{l}\text { I tell my partner that } \\
\text { his/her stress is not that } \\
\text { bad and help him/her to } \\
\text { see the situation in a } \\
\text { different light }\end{array}$ & 1 & 2 & 3 & 4 & 5 & 6 \\
\hline 30 & $\begin{array}{l}\text { I listen to my partner and } \\
\text { give him/her space to } \\
\text { communicate the entire } \\
\text { situation }\end{array}$ & 1 & 2 & 3 & 4 & 5 & 6 \\
\hline 31 & $\begin{array}{l}\text { I let my partner know } \\
\text { that I do not want to be } \\
\text { bothered with his/her } \\
\text { problems }\end{array}$ & 1 & 2 & 3 & 4 & 5 & 6 \\
\hline 32 & $\begin{array}{l}\text { I take my partner in my } \\
\text { arms and am tender } \\
\text { toward him/her }\end{array}$ & 1 & 2 & 3 & 4 & 5 & 6 \\
\hline 33 & $\begin{array}{l}\text { When my partner is } \\
\text { stressed, I tend to } \\
\text { withdraw }\end{array}$ & 1 & 2 & 3 & 4 & 5 & 6 \\
\hline 34 & $\begin{array}{l}\text { I console and say nice } \\
\text { things to make him/her } \\
\text { feel good }\end{array}$ & 1 & 2 & 3 & 4 & 5 & 6 \\
\hline 35 & $\begin{array}{l}\text { I tell my partner that } \\
\text { his/her way of handling } \\
\text { stress bothers me }\end{array}$ & 1 & 2 & 3 & 4 & 5 & 6 \\
\hline 36 & $\begin{array}{l}\text { I take on things that my } \\
\text { partner would normally } \\
\text { do in order to help } \\
\text { him/her out }\end{array}$ & 1 & 2 & 3 & 4 & 5 & 6 \\
\hline 37 & $\begin{array}{l}\text { Although I say nice } \\
\text { things and take him/her }\end{array}$ & 1 & 2 & 3 & 4 & 5 & 6 \\
\hline
\end{tabular}




\begin{tabular}{|c|c|c|c|c|c|c|c|}
\hline & $\begin{array}{l}\text { in my arms, my thoughts } \\
\text { are somewhere else }\end{array}$ & & & & & & \\
\hline 38 & $\begin{array}{l}\text { I show my partner that I } \\
\text { stand by him/her }\end{array}$ & 1 & 2 & 3 & 4 & 5 & 6 \\
\hline 39 & $\begin{array}{l}\text { When my partner heels } \\
\text { he/she has too much to } \\
\text { do, I help him/her out }\end{array}$ & 1 & 2 & 3 & 4 & 5 & 6 \\
\hline 40 & $\begin{array}{l}\text { I tell my partner when I } \\
\text { think he/she coped well } \\
\text { with stress and praise } \\
\text { him/her }\end{array}$ & 1 & 2 & 3 & 4 & 5 & 6 \\
\hline 41 & $\begin{array}{l}\text { I take into account when } \\
\text { my partner needs to } \\
\text { spend time alone and I } \\
\text { approach him/her later }\end{array}$ & 1 & 2 & 3 & 4 & 5 & 6 \\
\hline 42 & $\begin{array}{l}\text { Although I support my } \\
\text { partner, I am amazed at } \\
\text { those people who can } \\
\text { deal with their problems } \\
\text { themselves }\end{array}$ & 1 & 2 & 3 & 4 & 5 & 6 \\
\hline 43 & $\begin{array}{l}\text { I try to think through the } \\
\text { situation with my partner } \\
\text { and help/him her to } \\
\text { understand the problem }\end{array}$ & 1 & 2 & 3 & 4 & 5 & 6 \\
\hline 44 & $\begin{array}{l}\text { I do things for my partner } \\
\text { when he/she asks me to }\end{array}$ & 1 & 2 & 3 & 4 & 5 & 6 \\
\hline 45 & $\begin{array}{l}\text { I help my partner to relax } \\
\text { by giving him/her a } \\
\text { massage or something } \\
\text { like that }\end{array}$ & 1 & 2 & 3 & 4 & 5 & 6 \\
\hline 46 & $\begin{array}{l}\text { When my partner is } \\
\text { stressed, I tend to get out } \\
\text { of his/her way }\end{array}$ & 1 & 2 & 3 & 4 & 5 & 6 \\
\hline 47 & $\begin{array}{l}\text { My partner is satisfied } \\
\text { with the support I give to } \\
\text { him/her }\end{array}$ & 1 & 2 & 3 & 4 & 5 & 6 \\
\hline 48 & $\begin{array}{l}\text { The support I give to my } \\
\text { partner is helpful and } \\
\text { effective }\end{array}$ & 1 & 2 & 3 & 4 & 5 & 6 \\
\hline
\end{tabular}




\section{Section 4}

This section is about what you and your partner do when you are both feeling stressed

\begin{tabular}{|c|c|c|c|c|c|c|c|}
\hline & & Never & Rarely & Sometimes & Often & $\begin{array}{l}\text { Very } \\
\text { Often }\end{array}$ & Always \\
\hline 49 & $\begin{array}{l}\text { We are supportive of each } \\
\text { other and help one } \\
\text { another out }\end{array}$ & 1 & 2 & 3 & 4 & 5 & 6 \\
\hline 50 & $\begin{array}{l}\text { We divide tasks fairly so } \\
\text { that both of us have an } \\
\text { equal amount of work to } \\
\text { do }\end{array}$ & 1 & 2 & 3 & 4 & 5 & 6 \\
\hline 51 & $\begin{array}{l}\text { As a couple, we generally } \\
\text { solve our problems on our } \\
\text { own }\end{array}$ & 1 & 2 & 3 & 4 & 5 & 6 \\
\hline 52 & $\begin{array}{l}\text { We have a good laugh } \\
\text { together about it and take } \\
\text { it lightly }\end{array}$ & 1 & 2 & 3 & 4 & 5 & 6 \\
\hline 53 & $\begin{array}{l}\text { We engage in a serious } \\
\text { discussion about the } \\
\text { problem and think } \\
\text { through what has to be } \\
\text { done }\end{array}$ & 1 & 2 & 3 & 4 & 5 & 6 \\
\hline 54 & $\begin{array}{l}\text { We meditate or pray } \\
\text { together }\end{array}$ & 1 & 2 & 3 & 4 & 5 & 6 \\
\hline 55 & $\begin{array}{l}\text { We help one another to } \\
\text { put the problem in } \\
\text { perspective and see it in a } \\
\text { new light }\end{array}$ & 1 & 2 & 3 & 4 & 5 & 6 \\
\hline 56 & $\begin{array}{l}\text { We help each other relax } \\
\text { with such things like } \\
\text { massage, taking a bath } \\
\text { together, or listening to } \\
\text { music }\end{array}$ & 1 & 2 & 3 & 4 & 5 & 6 \\
\hline 57 & $\begin{array}{l}\text { We sit down to talk } \\
\text { together and share our } \\
\text { feelings }\end{array}$ & 1 & 2 & 3 & 4 & 5 & 6 \\
\hline 58 & $\begin{array}{l}\text { When we are both } \\
\text { stressed, we withdraw } \\
\text { and avoid each other }\end{array}$ & 1 & 2 & 3 & 4 & 5 & 6 \\
\hline 59 & $\begin{array}{l}\text { We caress one another } \\
\text { and make love }\end{array}$ & 1 & 2 & 3 & 4 & 5 & 6 \\
\hline 60 & $\begin{array}{l}\text { I am satisfied with the } \\
\text { way my partner and I deal } \\
\text { with stress together }\end{array}$ & 1 & 2 & 3 & 4 & 5 & 6 \\
\hline 61 & $\begin{array}{l}\text { I found as a couple, the } \\
\text { way we deal with stress } \\
\text { together is effective }\end{array}$ & 1 & 2 & 3 & 4 & 5 & 6 \\
\hline
\end{tabular}


PART IV. COMMUNICATION

Next to each statement below, circle the number of the response that best describes the way you feel about the statement

\begin{tabular}{|c|c|c|c|c|c|c|}
\hline & & $\begin{array}{l}\text { Definitely } \\
\text { False }\end{array}$ & $\begin{array}{l}\text { Usually } \\
\text { False }\end{array}$ & $\begin{array}{l}\text { Neither } \\
\text { True or } \\
\text { False }\end{array}$ & $\begin{array}{l}\text { Usually } \\
\text { True }\end{array}$ & $\begin{array}{l}\text { Definitely } \\
\text { True }\end{array}$ \\
\hline 1 & $\begin{array}{l}\text { It is very easy for me to express } \\
\text { all my true feelings to my } \\
\text { partner }\end{array}$ & 1 & 2 & 3 & 4 & 5 \\
\hline 2 & $\begin{array}{l}\text { When we are having a problem, } \\
\text { my partner often gives me the } \\
\text { silent treatment }\end{array}$ & 1 & 2 & 3 & 4 & 5 \\
\hline 3 & $\begin{array}{l}\text { My partner sometimes makes } \\
\text { comments which put me down }\end{array}$ & 1 & 2 & 3 & 4 & 5 \\
\hline 4 & $\begin{array}{l}\text { I am sometimes afraid to ask } \\
\text { my partner for what I want }\end{array}$ & 1 & 2 & 3 & 4 & 5 \\
\hline 5 & $\begin{array}{l}\text { I wish my partner were more } \\
\text { willing to share his/her feelings } \\
\text { with me }\end{array}$ & 1 & 2 & 3 & 4 & 5 \\
\hline 6 & $\begin{array}{l}\text { Sometimes I have trouble } \\
\text { believing everything my partner } \\
\text { tells me }\end{array}$ & 1 & 2 & 3 & 4 & 5 \\
\hline 7 & $\begin{array}{l}\text { I often do not tell my partner } \\
\text { what I am feeling because } \\
\text { he/she should already know }\end{array}$ & 1 & 2 & 3 & 4 & 5 \\
\hline 8 & $\begin{array}{l}\text { I am very satisfied with how } \\
\text { my partner and I talk with each } \\
\text { other }\end{array}$ & 1 & 2 & 3 & 4 & 5 \\
\hline 9 & $\begin{array}{l}\text { I do not always share negative } \\
\text { feelings I have about my } \\
\text { partner because I am afraid } \\
\text { he/she will be angry }\end{array}$ & 1 & 2 & 3 & 4 & 5 \\
\hline 10 & $\begin{array}{l}\text { My partner is always a good } \\
\text { listener }\end{array}$ & 1 & 2 & 3 & 4 & 5 \\
\hline
\end{tabular}

\section{BACKGROUND INFORMATION}

Please provide the following information about yourself. Remember that this and all other information will be kept strictly confidential

Age:

Number of Children:

Ethnic Background:

Asian

Caucasian

Native American/Indian

Afro-American

Latino

Other: 
Education:

Less than high school

$\square \quad$ High school graduate

$\square \quad$ High school plus some college

$\square$ College graduate

$\square \quad$ College plus some graduate hours

$\square$ Master's degree

$\square$ PhD. MD, JD

$\square$ Other:

Occupation:

- Unskilled labor

$\square$ Managerial

$\square$ Homemaker/parent

$\square$ Skilled labor

$\square$ Professional

$\square$ Other:

Income:

Less than $\$ 10,000$

$\square \quad \$ 10,000$ - \$29,999

$\square \quad \$ 30,000-\$ 24,999$

$\square \quad \$ 50,000-\$ 69,999$

$\square \quad \$ 70,000-\$ 89,999$

$\square \$ 90,000+$

Religious affiliation:

Catholic

$\square$ Protestant

$\square$ Jewish

$\square$ Atheist/Agnostic

$\square$ Other: 


\section{Health History:}

Has anyone in your family received treatment for mental illness?

Yes

$\square \quad$ No

If yes, how is the person related to you?

- Parent

$\square$ Sibling

$\square$ Grandparent

$\square \quad$ Other (please specify):

Have you ever received treatment for depression?

$\square$ Yes

$\square \quad$ No

Thank you very much for completing the questionnaire. Please remember to return your questionnaires in the separate envelopes 


\section{univenasiry of LOUISVILLE}

Human Subjects Protection Program Office

MedCenter One - Suite 200

501 E. Broadway

Louisville, KY 40202-1798

Office: 502.852 .5188 Fax: 502.852 .2164

DATE:

June 19, 2015

TO:

Karen Kayser

FROM:

The University of Louisville Institutional Review Board

IRB NUMBER:

15.0571

STUDY TITLE:

Younger Couples Coping with Breast Cancer: Examining the Relationship between Mutuality, Dyadic Coping, and Quality of Life of Patients and

REFERENCE \#: Partners

IRB STAFF CONTACT:

$$
365202
$$

Jackie Powell, CIP

$852-4101$

This study was reviewed on 06/18/2015 by the Chair of the Institutional Review Board and approved through the Expedited Review Procedure, according to 45 CFR 46.110(b), since this study falls under Category 5: Research involving materials (data, documents, records, or specimens) that have been collected, or will be collected solely for non-research purposes (such as medical treatment or diagnosis).

This study was also approved through 45 CFR 46.116 (D), which means that it has been granted a waiver of informed consent because it meets the following criteria:

- The research involves no more than minimal risk to the subjects.

- The waiver or alteration will not adversely affect the rights and welfare of the subjects.

- The research could not practicably be carried out without the waiver or alteration.

- Whenever appropriate, the subjects will be provided with the additional pertinent information after participation.

The following items have been approved:

\begin{tabular}{|l|l|l|l|}
\hline Submission Components \\
\hline Study Protocol and Survey & Version 1.0 & $06 / 16 / 2015$ & Approved \\
\hline Data File Overview & Version 1.0 & $06 / 16 / 2015$ & Approved \\
\hline
\end{tabular}

This study now has final IRB approval from 06/18/2015 through 06/17/2016. 


\section{Bivariate Analyses: Patients}

Table 1B. Correlations of the Major Study Variables Patients $(n=86)$

\begin{tabular}{|c|c|c|c|c|c|c|c|c|c|c|c|c|c|c|}
\hline Variables & 12 & 3 & 4 & 5 & 6 & 7 & 8 & 9 & 10 & 11 & 12 & 13 & 14 & 15 \\
\hline 1. Stress Communication & $.54 * * *$ & $.59 * * *$ & -.14 & $-.36 * *$ & $.65 * * *$ & $.36 * *$ & $.33 * *$ & -.14 & $.32 * *$ & -.19 & -.02 & -.04 & .02 & -.03 \\
\hline 2. Common Dyadic Coping & & $.90 * * *$ & $-.43 * * *$ & $-.57 * *$ & $.87 * * *$ & $.42 * * *$ & $.39 * * *$ & .06 & $.26 *$ & -.08 & .06 & .09 & .07 & .10 \\
\hline 3. Positive Dyadic Coping & & & $-.47 * * *$ & $-.58 * * *$ & $.95 * * *$ & $.51 * * *$ & $.47 * * *$ & -.08 & $.30 * *$ & -.17 & -.03 & -.01 & -.04 & -.01 \\
\hline 4. Hostile Dyadic Coping & & & & $.34 * *$ & $-.28 * *$ & $-.44 * * *$ & $-.39 * * *$ & -.17 & $-.35 * *$ & -.06 & -.11 & $-.32 *$ & $-.22 *$ & $-.27 * *$ \\
\hline 5. Avoidance of Dyadic Coping & & & & & $-.48 * * *$ & $-.37 * * *$ & $-.45 * * *$ & -.01 & $-.27 *$ & .04 & -.13 & -.10 & -.01 & -.09 \\
\hline 6. Total Dyadic Coping & & & & & & $.41 * * *$ & $.40 * * *$ & -.12 & $.23 *$ & $-.21 *$ & -.09 & -.08 & -.11 & -.09 \\
\hline 7. Mutuality & & & & & & & $.70 * * *$ & .04 & $.62 * * *$ & .01 & $.24 *$ & $.30 * *$ & $.12 *$ & $.27 *$ \\
\hline 8. Communication & & & & & & & & -.02 & $.49 * * *$ & -.03 & .10 & .14 & .11 & .15 \\
\hline 9. Physical Well-being & & & & & & & & & .18 & $.33 * *$ & $.59 * * *$ & $.73 * * *$ & $.60 * * *$ & $.75 * * *$ \\
\hline 10. Social Well-being & & & & & & & & & & .16 & $.38 * * *$ & $.56 * * *$ & $.29 * *$ & $.53 * * *$ \\
\hline 11. Emotional Well-being & & & & & & & & & & & $.48 * * *$ & $.62 * * *$ & $.35 * *$ & $.59 * * *$ \\
\hline 12. Functional Well-being & & & & & & & & & & & & $.88 * * *$ & $.49 * * *$ & $.85 * * *$ \\
\hline 13. FACT-G & & & & & & & & & & & & & $.60 * * *$ & $.76 * * *$ \\
\hline 14. Breast Cancer Symptoms & & & & & & & & & & & & & & $.97 * * *$ \\
\hline 15. FACT-B & & & & & & & & & & & & & & \\
\hline
\end{tabular}


Table 2B. Correlations Socio-Demographic Characteristics and Major Study Variables Patients ( $\mathrm{n}=86$ )

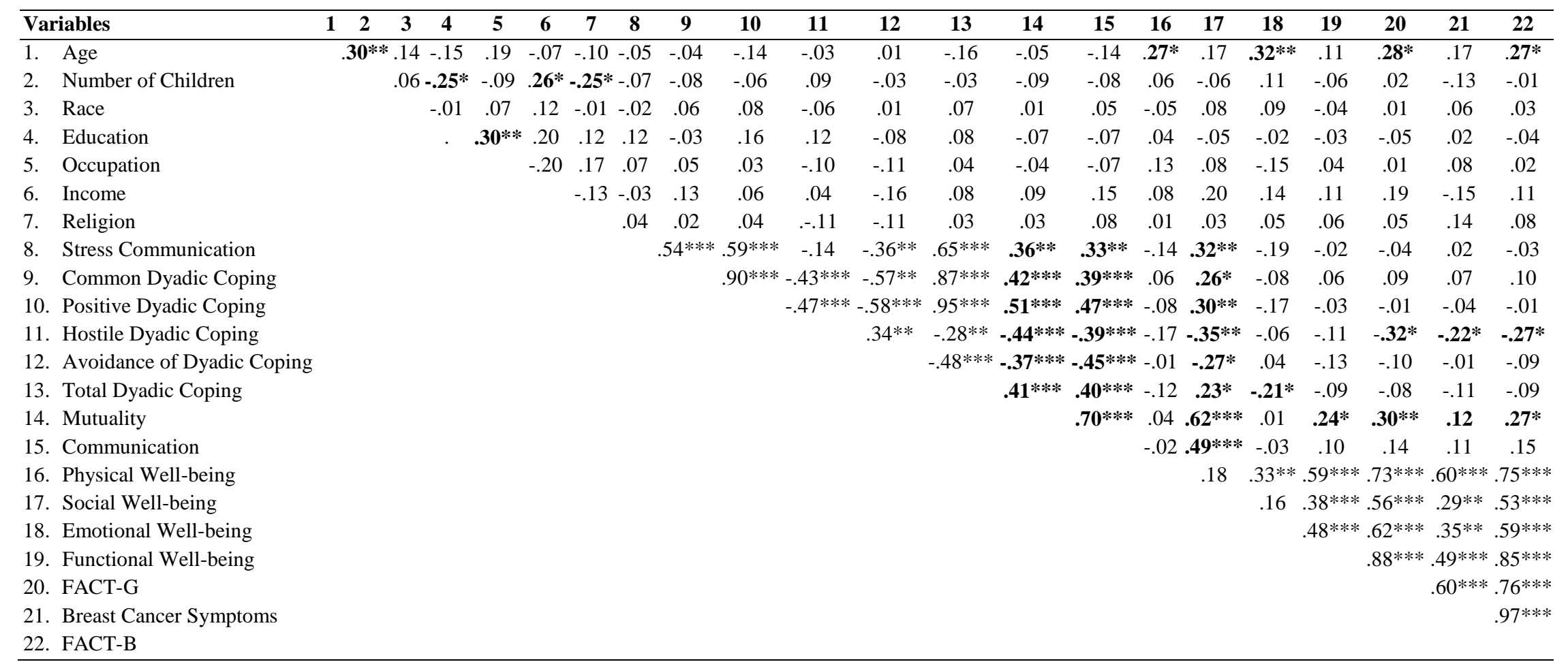


Table 3B. Correlations Relational Variables and Major Study Variables Patients $(n=86)$

\begin{tabular}{|c|c|c|c|c|c|c|c|c|c|c|c|c|c|c|c|c|}
\hline Variable & 12 & 3 & 4 & 5 & 6 & 7 & 8 & 9 & 10 & 11 & 12 & 13 & 14 & 15 & 16 & 17 \\
\hline 1. Marital Status & $.27 *$ & .07 & -.09 & -.06 & -.11 & -.10 & -.05 & -.04 & .14 & -.06 & .14 & .11 & -.05 & .05 & -.06 & .01 \\
\hline 2. Length of Relationship & & -.02 & -.03 & -.11 & -.07 & -.07 & -.13 & -.01 & -.04 & $.25 *$ & $.21 *$ & $.28 *$ & .18 & $.31 * *$ & .12 & $.28 *$ \\
\hline 3. Stress Communication & & & $.54 * * *$ & $.59 * * *$ & -.14 & $-.36 * *$ & $.65 * * *$ & $.36 * *$ & $.33 * *$ & -.14 & $.32 * *$ & -.19 & -.02 & -.04 & .02 & -.03 \\
\hline 4. Common Dyadic Coping & & & & $.90 * * *$ & $-.43 * * *$ & $-.57 * *$ & $.87 * * *$ & $.42 * * *$ & $.39 * * *$ & .06 & $.26^{*}$ & -.08 & .06 & .09 & .07 & .10 \\
\hline 5. Positive Dyadic Coping & & & & & $-.47 * * *$ & $-.58 * * *$ & $.95 * * *$ & $.51 * * *$ & $.47 * * *$ & -.08 & $.30 * *$ & -.17 & -.03 & -.01 & -.04 & -.01 \\
\hline 6. Hostile Dyadic Coping & & & & & & $.34 * *$ & $-.28 * *$ & $-.44 * * *$ & $-.39 * * *$ & -.17 & $-.35 * *$ & -.06 & 11 & $-.22 *$ & $-.32 * *$ & $-.27 *$ \\
\hline 7. Avoidance of Dyadic Coping & & & & & & & $-.48 * * *$ & $-.37 * * *$ & $-.45 * * *$ & -.01 & $-.27 *$ & .04 & -.13 & -.10 & -.01 & -.09 \\
\hline 8. Total Dyadic Coping & & & & & & & & $.41 * * *$ & $.40 * * *$ & -.12 & $.23 *$ & $-.21 *$ & -.09 & -.08 & -.11 & -.09 \\
\hline 9. Mutuality & & & & & & & & & $.70 * * *$ & .04 & $.62 * * *$ & .01 & $.24^{*}$ & $.30 * *$ & .12 & $.28 *$ \\
\hline 10. Communication & & & & & & & & & & -.02 & $.49 * * *$ & -.03 & .10 & .14 & .11 & .15 \\
\hline 11. Physical Well-being & & & & & & & & & & & .18 & $.33 * *$ & $.59 * * *$ & $.73 * * *$ & $.60 * * *$ & $.75 * * *$ \\
\hline 12. Social Well-being & & & & & & & & & & & & .16 & $.38 * * *$ & $.56 * * *$ & $.29 * *$ & $.53 * * *$ \\
\hline 13. Emotional Well-being & & & & & & & & & & & & & $.48 * * *$ & $.62 * * *$ & $.35^{* *}$ & $.59 * * *$ \\
\hline 14. Functional Well-being & & & & & & & & & & & & & & $.88 * * *$ & $.49 * * *$ & $.85 * * *$ \\
\hline 15. FACT-G & & & & & & & & & & & & & & & $.60 * * *$ & $.97 * * *$ \\
\hline 16. Breast Cancer Symptoms & & & & & & & & & & & & & & & & $.76 * * *$ \\
\hline 17. FACT-B & & & & & & & & & & & & & & & & \\
\hline
\end{tabular}


Table 4B. Correlations Clinical Variables and Major Study Variables Patients $(n=86)$

\begin{tabular}{|c|c|c|c|c|c|c|c|c|c|c|c|c|c|c|c|c|c|}
\hline Variable & 1 & 2 & 3 & 4 & 5 & 6 & 7 & 8 & 9 & 10 & 11 & 12 & 13 & 14 & 15 & 16 & 17 \\
\hline 1. Medications & & $.36^{* *}$ & .14 & -.03 & .04 & $.26^{*}$ & .21 & .09 & -.01 & -.17 & $-.32^{* *}$ & -.05 & -.06 & $-.24^{*}$ & $-.26^{*}$ &.$- .36^{* *}$ & $-.31 *$ \\
\hline 2. Chemotherapy & & & -.01 & -.09 & -.06 & .16 & .01 & -.06 & .03 & .05 & $-.33 * *$ & -.02 & -.13 & $-.22 *$ & $-.26 *$ & $-.32 * *$ & $-.30 * *$ \\
\hline 3. Stress Communication & & & & $.54 * * *$ & $.59 * * *$ & -.14 & $-.36 * *$ & $.65^{* * *}$ & $.36 * *$ & $.33 * *$ & -.14 & $.32 * *$ & -.19 & -.02 & -.04 & .02 & -.03 \\
\hline 4. Common Dyadic Coping & & & & & $.90 * * *$ & $-.43 * * *$ & $-.57 * *$ & $.87 * * *$ & $.42 * * *$ & $.39 * * *$ & .06 & $.26^{*}$ & -.08 & .06 & .09 & .07 & .10 \\
\hline 5. Positive Dyadic Coping & . & & & & & $-.47 * * *$ & $-.58 * * *$ & $.95 * * *$ & $.51 * * *$ & $.47 * * *$ & -.08 & $.30 * *$ & -.17 & -.03 & -.01 & -.04 & -.01 \\
\hline 6. Hostile Dyadic Coping & & & & & & & $.34 * *$ & $-.28 * *$ & $-.44 * * *$ & $-.39 * * *$ & -.17 & $-.35 * *$ & -.06 & -.11 & $-.22 *$ & $-.33^{*}$ & $-.27 *$ \\
\hline 7. Avoidance of Dyadic Coping & & & & & & & & $-.48 * * *$ & $-.37 * * *$ & $-.45 * * *$ & -.01 & $-.27 *$ & .04 & -.13 & -.10 & -.01 & -.09 \\
\hline 8. Total Dyadic Coping & & & & & & & & & $.41 * * *$ & $.40 * * *$ & -.12 & $.23^{*}$ & $-.21 *$ & -.09 & -.08 & -.11 & -.09 \\
\hline 9. Mutuality & & & & & & & & & & $.70 * * *$ & .04 & $.62 * * *$ & .01 & $.24 *$ & $.30 * *$ & .12 & $.28 *$ \\
\hline 10. Communication & & & & & & & & & & & -.02 & $.49 * * *$ & -.03 & .10 & .14 & .11 & .15 \\
\hline 11. Physical Well-being & & & & & & & & & & & & .18 & $.33 * *$ & $.59 * * *$ & $.73 * * *$ & $.60 * * *$ & $.75 * * *$ \\
\hline 12. Social Well-being & & & & & & & & & & & & & .16 & $.38 * * *$ & $.56 * * *$ & $.29 * *$ & $.53 * * *$ \\
\hline 13. Emotional Well-being & & & & & & & & & & & & & & $.48 * * *$ & $.62 * * *$ & $.35^{* *}$ & $.59 * * *$ \\
\hline 14. Functional Well-being & & & & & & & & & & & & & & & $.88 * * *$ & $.49 * * *$ & $.85 * * *$ \\
\hline 15.FACT-G & & & & & & & & & & & & & & & & $.60 * * *$ & $.97 * * *$ \\
\hline 16. Breast Cancer Symptoms & & & & & & & & & & & & & & & & & $.76^{* * *}$ \\
\hline 17.FACT-B & & & & & & & & & & & & & & & & & \\
\hline
\end{tabular}


Table 5B. Correlations Mental Health and Major Study Variables Patients $(n=86)$

\begin{tabular}{|c|c|c|c|c|c|c|c|c|c|c|c|c|c|c|c|c|c|c|c|}
\hline Variable & 1 & 2 & 3 & 4 & 5 & 6 & 7 & 8 & 9 & 10 & 11 & 12 & 13 & 14 & 15 & 16 & 17 & 18 & 19 \\
\hline 1. Mental Health Treatment & & $-.78 * * *$ & $.35^{*}$ & $-.37 * *$ & .20 & -.12 & -.06 & .04 & .06 & -.03 & .01 & -.06 & -.05 & .02 & .06 & .03 & .02 & -.06 & -.01 \\
\hline $\begin{array}{l}\text { 2. Relationship to person who } \\
\text { received treatment }\end{array}$ & & & $-.26 *$ & $.29 *$ & -.09 & .04 & .01 & .02 & .07 & .02 & -.08 & .01 & -.09 & -.09 & -.15 & -.15 & -.17 & .02 & -.12 \\
\hline 3. Treatment for Depression & & & & $-.88 * * *$ & .11 & -.16 &.-.10 & .01 & .08 & -.10 & -.16 & -.15 & -.08 & .11 & -.09 & $-.27 *$ & $-.22 *$ & -.05 & -.18 \\
\hline 4. Timing of Diagnosis & & & & & -.09 & .23 & .21 & -.08 & -.09 & .20 & .15 & .09 & .90 & .09 & .07 & .18 & .16 & .02 & .13 \\
\hline 5. Stress Communication & & & & & . & $.54 * * *$ & $.59 * * *$ & -.14 & $-.36 * *$ & $.65 * * *$ & $.36^{* *}$ & $.33 * *$ & -.14 & $.32 * *$ & -.19 & -.02 & -.04 & .02 & -.03 \\
\hline 6. Common Dyadic Coping & & & & & & & $.90 * * *$ & $-.43^{* * *}$ & $-.57 * *$ & $.87 * * *$ & $.42 * * *$ & $.39 * * *$ & .06 & $.26^{*}$ & -.08 & .06 & .09 & .07 & .10 \\
\hline 7. Positive Dyadic Coping & & & & & & & & $-.47 * * *$ & $-.58 * * *$ & $.95 * * *$ & $.51 * * *$ & $.47 * * *$ & -.08 & $.30 * *$ & -.17 & -.03 & -.01 & -.04 & -.01 \\
\hline 8. Hostile Dyadic Coping & & & & & & & & & $.34 * *$ & $-.28 * *$ & $-.44 * * *$ & $-.39 * * *$ & -.17 & $-.35 * *$ & -.06 & -.11 & $-.22 *$ & $-.32 * *$ & $-.27 *$ \\
\hline 9. Avoidance of Dyadic Coping & & & & & & & & & & $-.48 * * *$ & $-.37 * * *$ & $-.45 * * *$ & -.01 & $-.27 *$ & .04 & -.13 & -.10 & -.01 & -.09 \\
\hline 10.Total Dyadic Coping & & & & & & & & & & & $.41 * * *$ & $.40 * * *$ & -.12 & $.23 *$ & $-.21 *$ & -.09 & -.08 & -.11 & -.09 \\
\hline 11.Mutuality & & & & & & & & & & & & $.70 * * *$ & .04 & $.62 * * *$ & .01 & $.24 *$ & $.30 * *$ & .12 & $.28 *$ \\
\hline 12.Communication & & & & & & & & & & & & & -.02 & $.49 * * *$ & -.03 & .10 & .14 & .11 & .15 \\
\hline 13.Physical Well-being & & & & & & & & & & & & & & .18 & $.33^{* *}$ & $.59 * * *$ & $.73 * * *$ & $.60 * * *$ & $.75^{* * *}$ \\
\hline 14.Social Well-being & & & & & & & & & & & & & & & .16 & $.38 * * *$ & $.56^{* * *}$ & $.29 * *$ & $.53 * * *$ \\
\hline 15.Emotional Well-being & & & & & & & & & & & & & & & & $.48 * * *$ & $.62 * * *$ & $.35 * *$ & $.59 * * *$ \\
\hline 16.Functional Well-being & & & & & & & & & & & & & & & & & $.88 * * *$ & $.49 * * *$ & $.85^{* * *}$ \\
\hline 17.FACT-G & & & & & & & & & & & & & & & & & & $.60 * * *$ & $.97 * * *$ \\
\hline 18. Breast Cancer Symptoms & & & & & & & & & & & & & & & & & & & $.76^{* * *}$ \\
\hline 19.FACT-B & & & & & & & & & & & & & & & & & & & \\
\hline
\end{tabular}




\section{Bivariate Analyses: Partners}

Table 6B. Correlations of the Major Study Variables Partners $(n=86)$

\begin{tabular}{|c|c|c|c|c|c|c|c|c|c|c|}
\hline Variable & 1 & 2 & 3 & 4 & 5 & 6 & 7 & 8 & 9 & 10 \\
\hline 1. Stress Communication & & $.44 * * *$ & $.43 * * *$ & -.02 & -.17 & $.60 * * *$ & $.30 * *$ & $.21 *$ & -.02 & -.07 \\
\hline 2. Common Dyadic Coping & & & $.93 * * *$ & $-.41 * * *$ & $-.45 * * *$ & $.90 * * *$. & $.75 * * *$ & $.67 * * *$ & .06 & -.09 \\
\hline 3. Positive Dyadic Coping & & & & $-.45 * * *$ & $-.54 * * *$ & $.94 * * *$. & $.76 * * *$ & $.65 * * *$ & .09 & -.09 \\
\hline 4. Hostile Dyadic Coping & & & & & $.49 * * *$ & $-.22 *$ & $-.57 * * *$ & $-.54 * * *$ & $-.31 * *$ & $.37 * * *$ \\
\hline 5. Avoidance of Dyadic Coping & & & & & & $-.36 * *$ & $-.58 * * *$ & $-.63 * * *$ & $-.21 *$ & .08 \\
\hline 6. Total Dyadic Coping & & & & & & & $.65 * * *$ & $.53 * * *$ & .01 & -.02 \\
\hline 7. Mutuality & & & & & & & & $.75 * * *$ & .10 &.-18 \\
\hline 8. Communication & & & & & & & & & .14 & -.11 \\
\hline 9. Emotional Wellbeing & & & & & & & & & & $-.45 * * *$ \\
\hline 10. Illness Intrusiveness & & & & & & & & & & \\
\hline
\end{tabular}


Table 7B. Correlations Socio-Demographic Characteristics and Major Study Variables Partners (n=86)

\begin{tabular}{|c|c|c|c|c|c|c|c|c|c|c|c|c|c|c|c|c|}
\hline Variable & 2 & 3 & 4 & 5 & 6 & 7 & 8 & 9 & 10 & 11 & 12 & 13 & 14 & 15 & 16 & 17 \\
\hline 1. Age & $.35 * *$ & -.11 & .12 & -.12 & -.04 & -.10 & .10 & .13 & .17 & $-.24 *$ & -.08 & .14 & .03 & -.02 & $.34 * *$ & $-.42 * * *$ \\
\hline 2. Number of Children & & $-.34 * *$ & -.07 & -.02 & $.22 *$ & $-.24 *$ & .06 & .11 & .12 & -.17 & -.15 & .05 & .18 & .15 & .13 & -.20 \\
\hline 3. Race & & & .03 & -.04 & -.18 & .05 & -.09 & -.13 & -.14 & .01 & .01 & -.15 & -.04 & -.10 & -.01 & -.01 \\
\hline 4. Education & & & & .13 & .19 & $.37 * *$ & $.21 *$ & .17 & .16 & .06 & -.10 & .19 & .08 & .16 & .09 & .03 \\
\hline 5. Occupation & & & & & -.01 & .06 & -.07 & -.08 & -.02 & .06 & -.17 & -.04 & -.05 & -.04 & .05 & $.24 *$ \\
\hline 6. Income & & & & & & -.04 & -.08 & -.04 & -.04 & -.04 & .03 & -.08 & -.04 & .06 & .08 & .06 \\
\hline 7. Religion & & & & & & & -.08 & -.15 & -.12 & .08 & -.06 & -.14 & -.16 & -.13 & .09 & -.03 \\
\hline 8. Stress Communication & & & & & & & & $.44 * * *$ & $.43 * * *$ & -.02 & -.17 & $.60 * * *$ & $.30 * *$ & $.21 *$ & -.02 & -.07 \\
\hline $\begin{array}{l}\text { 9. Common Dyadic } \\
\text { Coping }\end{array}$ & & & & & & & & & $.93 * * *$ & $-.41 * * *$ & $-.45 * * *$ & $.90 * * *$. & $.75 * * *$ & $.67 * * *$ & .06 & -.09 \\
\hline $\begin{array}{l}\text { 10. Positive Dyadic } \\
\text { Coping }\end{array}$ & & & & & & & & & & $-.45 * * *$ & $-.54 * * *$ & $.94 * * *$ & $.76 * * *$ & $.65 * * *$ & .09 & -.09 \\
\hline 11. Hostile Dyadic Coping & & & & & & & & & & & $.49 * * *$ & $-.22 *$ & $-.57 * * *$ & $-.54 * * *$ & $-.31 * *$ & $.37 * * *$ \\
\hline $\begin{array}{l}\text { 12. Avoidance of Dyadic } \\
\text { Coping }\end{array}$ & & & & & & & & & & & & $-.36^{* *}$ & $-.58 * * *$ & $-.63 * * *$ & $-.21 *$ & .08 \\
\hline 13. Total Dyadic Coping & & & & & & & & & & & & & $.65 * * *$ & $.53 * * *$ & .01 & -.02 \\
\hline 14. Mutuality & & & & & & & & & & & & & & $.75 * * *$ & .10 &.-18 \\
\hline 15. Communication & & & & & & & & & & & & & & & .14 & -.11 \\
\hline 16. Emotional Well-being & & & & & & & & & & & & & & & & $-.45 * * *$ \\
\hline 17. Illness Intrusiveness & & & & & & & & & & & & & & & & \\
\hline
\end{tabular}


Table 8B. Correlations Relational Variables and Major Study Variables Partners ( $n=86)$

\begin{tabular}{|c|c|c|c|c|c|c|c|c|c|c|c|c|}
\hline Variable & 1 & 2 & 3 & 4 & 5 & 6 & 7 & 8 & 9 & 10 & 11 & 12 \\
\hline 1. Marital Status & & $.27 *$ & -.08 & -.01 & .01 & .06 & .02 & .01 & -.04 & .03 & -.03 & .14 \\
\hline 2. Length of Relationship & & & .06 & .16 & .18 & $-.27 *$ & -.13 & .13 & .04 & .02 & $.26^{*}$ & $-.24 *$ \\
\hline 3. Stress Communication & & & & $.44 * * *$ & $.43 * * *$ & -.02 & -.17 & $.60 * * *$ & $.30 * *$ & $.21 *$ & -.02 & -.07 \\
\hline 4. Common Dyadic Coping & & & & & $.93 * * *$. & $-.41 * * *$ & $-.45 * * *$ & $.90 * * *$ & $.75 * * *$ & $.67 * * *$ & .06 & -.09 \\
\hline 5. Positive Dyadic Coping & & & & & & $-.45 * * *$ & $-.54 * * *$ & $.94 * * *$ & $.76^{* * *}$ & $.65^{* * *}$ & .09 & -.09 \\
\hline 6. Hostile Dyadic Coping & & & & & & & $.49 * * *$ & $-.22 *$ & $-.57 * * *$ & $-.54 * * *$ & $-.31 * *$ & $.37 * * *$ \\
\hline 7. Avoidance of Dyadic Coping & & & & & & & & $-.36^{* *}$ & $-.58 * * *$ & $-.63^{* * *}$ & $-.21 *$ & .08 \\
\hline 8. Total Dyadic Coping & & & & & & & & & $.65 * * *$ & $.53 * * *$ & .01 & -.02 \\
\hline 9. Mutuality & & & & & & & & & & $.75 * * *$ & .10 &.-.18 \\
\hline 10. Communication & & & & & & & & & & & .14 & -.11 \\
\hline 11.Emotional Well-being & & & & & & & & & & & & $-.45 * * *$ \\
\hline 12. Illness Intrusiveness & & & & & & & & & & & & \\
\hline
\end{tabular}


Table 9B. Correlations Mental Health and Major Study Variables Partners $(n=86)$

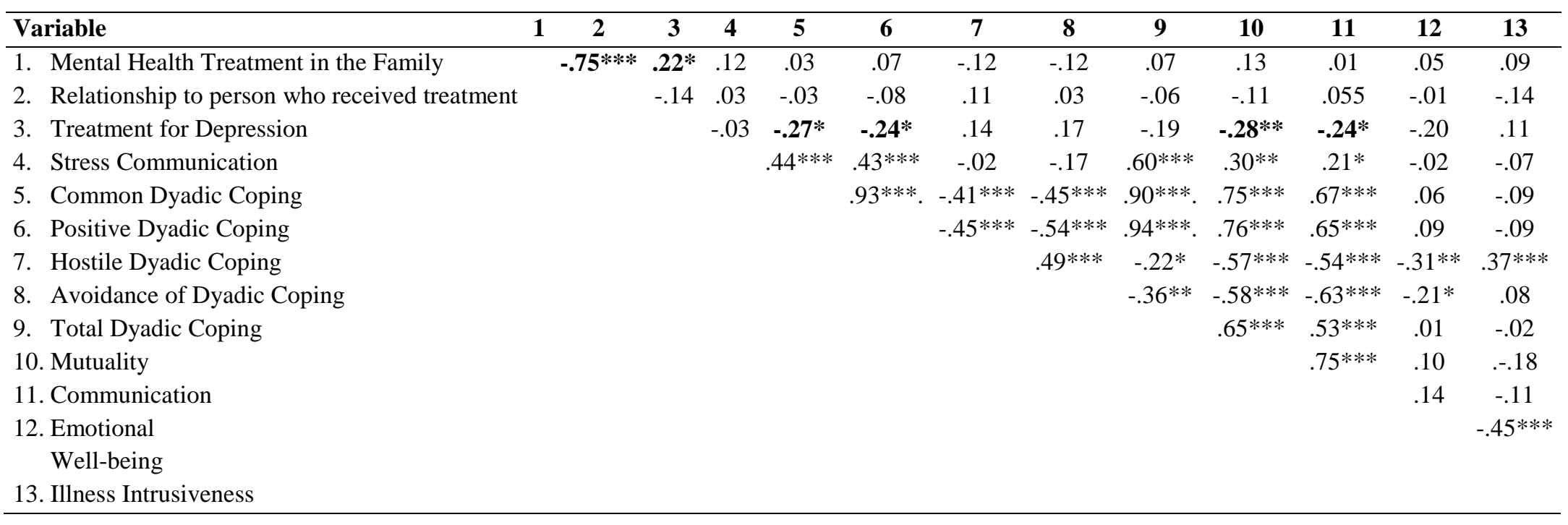




\section{APIM Model Calculations}

\section{Stress Communication}

Test of distinguishability:

Deviance of indistinguishable model $=402.538$, \# parameters $=5$

Deviance of distinguishable model $=358.818$, \# parameters $=9$

$\chi_{(4)}^{2}=43.72 \mathrm{p}<.001$

Pseudo R-squared:

Patient pseudo R-squared $=1-(0.437613 / 0.527515)=1704254855$

Caregivers pseudo R-squared $=1-(.508335 / .539096)=0.057060338$

Deviance test:

$\chi^{2}(4)=21.04, p<.001$

\section{Common Dyadic Coping}

Test of distinguishability:

Deviance of indistinguishable model $=241.457 \#$ parameters $=5$

Deviance of distinguishable model $=234.271$, \# parameters $=9$

$\chi^{2}(4)=7.186, p>.05$

Pseudo R-squared:

Patients pseudo R-squared $=1-(.245377 / .550773)=0.5544861495=55 \%$

Caregivers pseudo R-squared $=1-(.239807 / .393337)=0.3903268698=39 \%$

Deviance test:

$\chi^{2}(4)=99.42, \mathrm{p}<.001$

\section{Positive Dyadic Coping}

\section{Test of distinguishability:}

Deviance of indistinguishable model $=256.956$, \# parameters $=5$

Deviance of distinguishable model $=242.877$, \# parameters $=9$

$\chi^{2}(4)=14.08, \mathrm{p}<.01$

Pseudo R-squared:

Patients pseudo R-squared $=1-(.226486 / .642313)=0.64738=64.7 \%$

Caregivers pseudo R-squared $=1-(.270412 / .453944)=0.404305=40.3 \%$

Deviance test:

$\chi^{2}(4)=124.1, \mathrm{p}<0.001$

\section{Hostile Dyadic Coping}

\section{Test of distinguishability:}

Deviance of indistinguishable model $=247.313$, \# parameters $=5$

Deviance of distinguishable model $=230.365$, \# parameters $=9$

$\chi^{2}(4)=16.95, p<.01$

Pseudo R-Squared:

Patients pseudo R-squared $=1-(.297522 / .448406)=0.336489$

Caregivers pseudo R-squared $=1-(.168289 / .234436)=0.282153765$

Deviance test:

$\chi^{2}(4)=61.81 \mathrm{p}<0.001$ 


\section{Avoidance of Dyadic Coping}

Test of distinguishability:

Deviance of indistinguishable model $=375.213$, \# parameters $=5$

Deviance of distinguishable model $=364.694$, \# parameters $=9$

$\chi_{(4)}^{2}=10.51, \mathrm{p}<.05$

Pseudo R-Squared:

Patients pseudo R-squared $=1-(.650141 / .856634)=0.240516043$

Caregivers pseudo R-squared $=1-(.366211 / .503035)=0.2719969783$

Deviance test:

$\chi^{2}(4)=47.116 \mathrm{p}<0.001$

\section{Actor Partner Model by Age Group Calculation}

\section{Stress Communication Younger Couples}

Test of distinguishability:

Deviance of indistinguishable model $=156.22$, \# parameters $=5$

Deviance of distinguishable model $=135.369$, \# parameters $=9$

$\chi^{2}(4)=20.851, p<.001$

Pseudo $\mathbf{R}^{2}$ Calculation

Patients pseudo R-squared $=1-(.471552 / .632620)=0.25460466$

Caregivers pseudo R-squared $=1-(.355794 / .476539)=.2533790519$

Deviance test:

$\chi^{2}(4)=19.531, p<.001$

\section{Stress Communication Older Couples}

Test of distinguishability:

Deviance of indistinguishable model $=240.768 \#$ parameters $=5$

Deviance of distinguishable model $=213.372$, \# parameters $=9$

$\chi_{(4)}^{2}=27.39, \mathrm{p}<.001$

Pseudo R $\mathbf{R}^{2}$

Patients pseudo R-squared $=1-(.402495 / .451273)=0.1080897$

Caregivers pseudo R-squared $=1-(.562281 / .575937)=0.0237109267$

Deviance test:

$\chi_{(4)}^{2}=6.958, \mathrm{p}>.05$

\section{Common Dyadic Coping Younger Couples}

Test of distinguishability:

Deviance of indistinguishable model $=83.347$, \# parameters $=5$

Deviance of distinguishable model $=73.796$, \# parameters $=9$

$\chi_{(4)}^{2}=9.55, \mathrm{p}<.05$

Pseudo $\mathbf{R}^{2}$ :

Patients pseudo R-squared $=1-(.249183 / .557419)=.55297$

Caregivers pseudo R-squared $=1-(.135984 / .263804)=.484526$

Deviance test:

$\chi^{2}(4)=44.794, \mathrm{p}<.001$ 


\section{Common Dyadic Coping Older Couples}

Test of distinguishability:

Deviance of indistinguishable model $=155.017$, \# parameters $=5$

Deviance of distinguishable model $=-148.796$, \# parameters $=9$

$\chi_{(4)}^{2}=6.221, \mathrm{p}>.05$

Pseudo $\mathbf{R}^{2}$ :

Patients pseudo R-squared $=1-(.230922 / .538032)=.5707795$

Caregivers pseudo R-squared $=1-(.300280 / .475479)=.3684684287$

Deviance test:

$\chi_{(4)}^{2}=57.812 ., \mathrm{p}<.001$

\section{Positive Dyadic Coping Younger Couples}

Test of distinguishability:

Deviance of indistinguishable model $=79.412$, \# parameters $=5$

Deviance of distinguishable model $=67.421$, \# parameters $=9$

$\mathrm{X}^{2}(4)=11.99 \mathrm{p}<.05$

Pseudo $R^{2}$ :

Patients pseudo R-squared $=1-(.197209 / .654071)=.6984899193$

Caregivers pseudo R-squared $=1-(.139497 / .306525)=.549082457$

Deviance Test

$\chi^{2}(4)=67.408, p<.001$

\section{Positive Dyadic Coping Older Couples}

\section{Calculations:}

\section{Test of distinguishability:}

Deviance of indistinguishable model $=168.725$, \# parameters $=5$

Deviance of distinguishable model $=160.283$, \# parameters $=9$

$\mathrm{X}^{2}(4)=8.442, \mathrm{p}>.05$

Pseudo $\mathbf{R}^{2}$ :

Patient pseudo R-squared $=1-(.245146 / .633831)=.613231$

Caregiver pseudo R-squared $=1-(.339489 / .539157)=.62966$

Deviance Test:

$\chi^{2}(4)=66.025, \mathrm{p}<.001$

\section{Hostile Dyadic Coping Younger Couples}

\section{Test of distinguishability:}

Deviance of indistinguishable model $=92.049$, \# parameters $=5$

Deviance of distinguishable model $=73.654$, \# parameters $=9$

$\chi_{(4)}^{2}=18.395 \mathrm{p}<.01$

Pseudo R-squared:

Patients pseudo R-squared $=1-(.296508 / .734593)=.5963642$

Men pseudo R-squared $=1-(.095965 / .200454)=5212617359$

Deviance test:

$\chi_{(4)}^{2}=57.949, \mathrm{p}<.001$ 


\section{Hostile Dyadic Coping Older Couples}

\section{Test of distinguishability:}

Deviance of indistinguishable model $=104.922$, \# parameters $=5$

Deviance of distinguishable model $=108.124$, \# parameters $=9$

$\chi^{2}(4)=3.20, p>.05$

Pseudo $\mathbf{R}^{2}$ :

Patients pseudo R-squared $=1-(.171794 / .210745)=.1848252628$

Caregivers pseudo R-squared $=1-(.156147 / .215852)=.276601$

Deviance test:

$\chi_{(4)}^{2}=24.718, \mathrm{p}<.001$

\section{Avoidance of Dyadic Coping Younger Couples}

Test of distinguishability:

Deviance of indistinguishable model $=149.713$, \# parameters $=5$

Deviance of distinguishable model $=137.927$, \# parameters $=9$

$\chi^{2}(4)=11.786 \mathrm{p}<.05$

Pseudo R $\mathbf{R}^{2}$ :

Patient pseudo R-squared $=1-(.689037 / .978048)=.295497767$

Partners/ Caregivers pseudo R-squared $=1-(.258508 / .554739)=.5340006742$

Deviance test:

$\chi^{2}(4)=38.944, \mathrm{p}<.001$

\section{Avoidance of Dyadic Coping Older Couples}

Test of distinguishability:

Deviance of indistinguishable model $=215.286$, \# parameters $=5$

Deviance of distinguishable model $=210.482$, \# parameters $=9$

$\chi_{(4)}^{2}=4.804, \mathrm{p}>.05$

Pseudo $\mathbf{R}^{2}$ Calculation:

Patients pseudo R-squared $=1-(.580291 / .746721)=.2228811$

Partners pseudo R-squared $=1-(.370646 / .454526)=.1845438$

Deviance test:

$\chi^{2}(4)=19.879, \mathrm{p}<.001$ 


\title{
CURRICULUM VITAE
}

\author{
Chiara Acquati
}

Kent School of Social Work

2800 Shannon Drive

Patterson Hall, R. 203

University of Louisville

Louisville, KY, 40220

Louisville, KY, 40292

chiara.acquati@louisville.edu

857-756-8542

\section{EDUCATION}

Ph.D. Kent School of Social Work, University of Louisville, 2016

Dissertation: A Comparison of Younger and Older Couples Coping with Breast

Cancer: Examining the Relationship among Mutuality, Dyadic Coping, and

Quality of Life of Patients and Partners

Committee: Drs. Karen Kayser (chair), Wanda Collins, Eli Karam, Kristen Mark, Heehyul Moon

MSW Graduate School of Social Work, Boston College, Chestnut Hill, 2011

M.A. Department of Psychology, Catholic University of the Sacred Heart, Milan, 2007

B.A. Department of Psychology, Catholic University of the Sacred Heart, Milan, 2005

\section{PUBLICATIONS}

\section{Peer Reviewed Journal Articles}

2015 Zebrack, B., Kayser, K., Savas, S.A, Sundstrom, L., Henrickson, C, Acquati, C. \& Tamas, R. (2015). Psychosocial Distress Screening Implementation in Cancer Care: An Analysis of Adherence, Responsiveness, and Acceptability. Journal of Clinical Oncology, Apr 1, 1165-1170 doi:

http://jco.ascopubs.org/content/early/2015/02/18/JCO.2014.57.4020.abstract

Saita, E., Acquati, C., \& Kayser, K. Coping with Early Stage Breast Cancer: Examining the Influence of Personality Traits and Interpersonal Closeness.

Frontiers in Psychology: Psychology for Clinical Settings, doi: 10.3389/fpsyg.2015.00088

Saita, E., De Luca, L., \& Acquati, C. What is hope for breast cancer patients? A qualitative study. Mediterranean Journal of Clinical Psychology, 3(1), doi: 
http://cab.unime.it/journals/index.php/MJCP/issue/view/89/showToc

2014 Saita, E., Molgora, S., \& Acquati, C. Development and Evaluation of the Cancer Dyads Group Intervention: Preliminary Findings. Journal of Psychosocial Oncology, 32(6):647-64. doi:10.1080/07347332.2014.955242

2012 Kayser, K., Acquati, C., \& V. Tran, T. No Patients Left Behind: A Systematic Review of the Cultural Equivalence of Distress Screening Instruments. Journal of Psychosocial Oncology, 30(6), 679-693. doi:10.1080/07347332.2012.721489

\section{Submitted}

Acquati, C. \& Kayser, K. Psychosocial Predictors of Distress among Lung and Breast Cancer Patients. Frontiers in Psychology: Psychology for Clinical Settings

Saita, E., Acquati, C., Fenaroli. V., Zuliani, C., \& Bonanno, G. A Confirmatory Factor Analysis of the Perceived Ability to Cope with Trauma (PACT) Scale in an Italian Sample. TPM - Testing, Psychometrics, Methodology in Applied Psychology

\section{In Preparation}

Acquati, C., Zebrack, B., Faul, A., Meeske, K.A., Embry, L., Aguilar, C., Block, R., \& Hayes-Lattin, B. Sexual Functioning in Young Adult Cancer Patients: A 2Year Longitudinal Analysis. Target Journal: JAMA Pediatrics

Kayser, K. Acquati, C., Reese, J., Mark, K., Karam, E. \& Wittman, D. The Impact of Colorectal Cancer on the Couple Relationship: A Systematic Review. Target Journal: Supportive Care in Cancer

\section{Book Chapters}

2011 Kayser, K., Acquati, C., Saita, E. \& Costa, G. Coping Diadico e Relazione di Caregiving nella sfida alla malattia oncologica (Dyadic Coping and Caregiving while Facing Cancer). In E. Saita, Pensare alla salute e alla malattia: legami tra mente, corpo e contest di appartenenza. Percorsi di ricerca e di intervento. (89116)(Thinking of Health and Illness: Connections between Mind, Body and Cultural Context. Research and Intervention Pathways.). Milano: Educatt.

Kayser, K., Saita, E., \& Acquati, C. Famiglie, fonti di stress e capacita’ di fronteggiare gli eventi. Il caso della famiglia Americana (Families, Stress and Coping. The case of the American Family). In E. Saita, Pensare alla salute e alla malattia: legami tra mente, corpo e contest di appartenenza. Percorsi di ricerca e di intervento (69-88) (Thinking of Health and Illness: Connections between Mind, Body and Cultural Context. Research and Intervention Pathways). Milano: Educatt.

Kayser, K., Acquati, C. \& Saita, E. Coping e adattamento famigliare: Il ruolo dei fattori culturali (Coping and Family Adjustment: the Role of Cultural Factors). In E. Saita, Pensare alla salute e alla malattia: legami tra mente, corpo e contest di appartenenza. Percorsi di ricerca e di intervento. (117-134)(Thinking of Health and Illness: Connections between Mind, Body and Cultural Context. Research and Intervention Pathways). Milano: Educatt. 
2016 Saita, E., Zanini, S., Minetti, E., \& Acquati, C. Best Practices to Promote Patient and Donor Engagement to Care in Living Donor Transplant. In G. Graffigna (ed.) Promoting Patient Engagement and Participation for Effective Healthcare Reform, IGI-Global.

\section{In Preparation}

Acquati, C. \& Saita, E. Il coping diadico: La gestione degli eventi stressanti a livello di coppia. (Dyadic Coping: The Relational Management of Stressful Events)

Saita, E., Molgora, S., \& Acquati, C. The idiographic science perspective applied to the treatment of younger women with BRCA mutation

\section{FELLOWSHIPS \& AWARDS}

2015 American Cancer Society Doctoral Training Grant in Oncology Social Work $(\$ 20,000)$

2014 Faculty Favorite - University of Louisville Delphi Center for Teaching and Learning

2013 American Cancer Society Doctoral Training Grant in Oncology Social Work $(\$ 40,000)$

2012 Oncofertility Conference Award, Northwestern University (\$500)

AOSW Conference Scholarship, Association of Oncology Social Work (\$500)

ExCEL in Social Work Scholarship (\$500)

2011 Graduate Research Assistantship, University of Louisville School of Graduate and Interdisciplinary Studies

Academic Excellence Award, Graduate School of Social Work, Boston College

2009 Boston College Fellowship in Social Work

2008 The Researcher Fund Grant $(\$ 15,000)$

Enrico Legler Study Award, Legler Family Foundation $(\$ 2,000)$

\section{CONFERENCE ACTIVITY}

\section{Papers}

2017 Kayser, K., Wittman, D., Reese, J., Acquati, C., Hendren, S., \& Mark, K Couples' Coping with Impaired Sexual Functioning during and after Treatment for Rectal Cancer. $7^{\text {th }}$ Dyadic Coping Conference. January 17, Milan, Italy (accepted)

2016 Fenaroli, V., Molgora, S., Acquati, C., \& Saita, E. The Role of Dyadic Coping in the Transition to Parenthood. $8^{\text {th }}$ Congress of the European Society of Family Relations, August 31-September 3, Dortmund, Germany. (submitted) 
Acquati, C., Kayser, K., Moon, H., Mark, K., Karam. E., \& Collins, W. Older Couples Coping with Early-Stage Breast Cancer: A Cross-Sectional Examination of Mutuality, Dyadic Coping and Quality of Life. Gerontological Society of America Annual Scientific Meeting, November 16-20, New Orleans, LA (submitted)

2014 Zebrack, B., Kayser, K., Acquati, C., Sundstrom, L., Savas, S.A., McCoy, M. \& Tamas, R. Testing Adherence and Feasibility of Distress Screening Protocols: Implementing Best Practices in Psychosocial Care. 11th American Psychosocial Oncology Society (APOS) Conference, February 13-15 2014, Tampa

Zebrack, Z., Henrickson, C., Kayser, K., \& Acquati, C. Distress Screening Implementation: What to Do with the Data? 30th Association of Oncology Social Work (AOSW) Conference, May 28-30, Baltimore, MD

2013 Kayser, K., \& Acquati, C. Testing the Implementation of a Distress Screening Protocol: A Pilot Study. 29th Association of Oncology Social Work (AOSW) Conference, June 5-7, San Diego, CA

Acquati, C. Moving Towards the Future: Fresh Perspectives in Couples Coping with Cancer Research. Sixth International Meeting on Stress \& Coping. Couples Coping with Cancer-Related Stress: Translating Research to Practice, June 21-22, Louisville, KY

Acquati, C., Zebrack, B., Faul, A., Meeske, K.A., Embry, L., Aguilar, C., Block, R., Hayes-Lattin, B. \& Cole, S. Sex: A Lost Frontier for Adolescent and Young Adult (AYA) Cancer Patients. International Psycho-Oncology Society (IPOS) 15th World Congress, November 4-8, Rotterdam, The Netherlands.

2011 Kayser, K., Saita, E., \& Acquati, C. Women Coping with Breast Cancer: The Impact of Personality and Close Relationships on Coping Strategies. Society for Social Work and Research (SSWR) 15th Conference, January 12-16, Tampa, FL

Kayser, K., \& Acquati, C. Moderating Factors in the Relational Outcomes of Breast Cancer Patients in the Partners in Coping Program. International PsychoOncology Society (IPOS) 13th World Congress, 18-20 October, Antalya, Turkey.

2010 Kayser, K., Saita, E., \& Acquati, C. Women's Adaptation to Early Stage Breast Cancer: The Influence of Personality Traits and Interpersonal Closeness on the Style of Coping with Cancer. International Meeting on Stress and Coping in Close Relationships, February 18-19, Zurich, Switzerland.

\section{Posters}

2015 Valenti, F., Baratelli, G.M., Molgora, S., Acquati, C., Saita, E., \& Grassi, M.M. Il Fallimento della Diagnosi Precoce. Analisi dell'Esperienza di Donne Trattate per Tumore alla Mammella Localmente Avanzato. AIS - Attualità in Senologia, 4-6 November, Florence, Italy

Acquati, C. \& Kayser, K. Are We Responding to the Distress of Cancer Patients? An Examination of Psychosocial Response after Distress Screening. APOS-IPOS World Congress of Psycho-Oncology, 28 July - 1 August, Washington, DC 
Saita E., Acquati C., Molgora S., Valenti F., Grassi M. The Failure of Early Detection: The Experience of Women with Advanced Breast Cancer. APOS-IPOS World Congress of Psycho-Oncology, 28 July - 1 August, Washington, DC

Saita, E., Acquati, C. \& Irtelli, F. The Cancer Dyads Group Intervention to Promote Breast Cancer Patients' Adjustment, Coping and Perceived Social Support. APOS-IPOS World Congress of Psycho-Oncology, 28 July - 1 August, Washington, DC

2014 Saita, E., Jankovic, M., Rossetti, A., Zuliani, C. \& Acquati, C. Pictures of Me: The Framed Portrait Experience as a Psychological Intervention for Adolescent and Young Adult (AYA) Cancer Survivors. 11th American Psychosocial Oncology Society (APOS) Conference, February 13-15, Tampa, FL

Acquati, C., Zebrack, B., Faul, A., Meeske, K.A., Embry, L., Aguilar, C., Block, R., Hayes-Lattin, B. \& Cole, S. Sex - A Lost Frontier: Sexual Functioning in Adolescent and Young Adult (AYA) Cancer Patients in the Two Years Following Diagnosis. Critical Mass, November, Denver, CO

2013 Acquati, C., Saita, E., Kayser, K., Zuliani, C. \& Zanchi, D. Breast Cancer PreScreening Anxiety: Identifying Psychological Vulnerability among Italian Women. 10th American Psychosocial Oncology Society (APOS) Conference, 1416 February, Huntington Beach, CA

2012 Acquati, C. \& Kayser, K. Reproductive Distress in Female Cancer Survivors: A Systematic Review of Current Issues and Future Directions. 9th American Psychosocial Oncology Society (APOS) Conference, 23-25 February, Miami, FL

Kayser, K., Acquati, C., Gragg, H., Williams, K., \& Tamas, R. Psychological and Clinical Predictors of Self-Reported Distress among Breast Cancer Patients: An Exploratory Analysis of Distress Screening Scores. Research!Louisville, 18-20 September, Louisville, KY

Acquati, C., \& Kayser, K. Psychosocial Care for Young Adult Cancer Patients \& Survivors: From the Identification of Needs to the Development of Interventions for Cancer-Related Infertility \& Reproductive Health. Oncofertility Conference: Dialogues in Oncofertility, 27-28 September, Chicago, IL

2009 Saita, E., Negrini, L., \& Acquati, C. Il diario diadico di pazienti oncologici e dei loro caregiver: valutazione delle dinamiche relazionali (The dyadic diary of cancer patients and their caregivers: an evaluation of the relational dynamics.) 23rd Annual Conference of the European Health Psychology Society, 23 - 26 September, Pisa, Italy.

2008 Saita, E., Cigoli, V., Brognoli, S., \& Acquati, C. Il diario diadico come strumento di valutazione di un intervento clinico di gruppo rivolto a pazienti oncologici e caregiver (The dyadic diary as an evaluation tool of a group clinical intervention for cancer patients and their caregivers). National Congress of Health Psychology, October 1-4, Rovigo, Italy. 


\section{RESEARCH EXPERIENCE}

\section{University of Louisville, Kent School of Social Work}

2011-2015 Grant writing, data collection (individual and dyadic surveys), data management, data analysis and dissemination of results.

Studies:

2015 Younger Couples Coping with Breast Cancer: Examining the Relationship between Mutuality, Dyadic Coping and Quality of Life of Patients and Partners

2014 Couples Coping with Impaired Sexual Function during and after Treatment for Rectal Cancer

2013 Testing the Fidelity and Feasibility of Distress Screening Implementation in Routine Clinical Care with Cancer Patients

2012 Screening for Cancer Patients' Psychosocial Distress: A Descriptive Analysis of Distress Thermometer Scores at the James Graham Brown Cancer Center

\section{Catholic University of the Sacred Heart, Department of Psychology}

2006-2009 Data collection of individual and dyadic surveys and interviews, qualitative and quantitative data analysis, dissemination of findings

Coordinator and supervisor of research assistants for the project "From the Psychological Assessment to the Supportive-Expressive Group Therapy for Women with Breast Cancer and Their Caregivers. A Multi-Method Design"

Conductor of the Cancer Dyads Group Intervention for breast cancer patients and caregivers

Provided individual and couple therapy to breast cancer patients and caregivers

Liaison between the Oncology Team of the E. Bassini Hospital and the Catholic University of the Sacred Heart Department of Psychology

\section{TEACHING EXPERIENCE}

\section{University of Louisville, Kent School of Social Work, Sole Instructor}

Psychosocial Practice in Oncology I: Interpersonal Practice (fall 2013)

Psychosocial Practice in Oncology II: Community Approaches to Promote Health Equity (spring 2014)

Mental Health Specialization Integrative Seminar (fall 2014/spring 2015)

Practicum Faculty Liaison for Mental Health and Psychosocial Oncology MSSW Students (fall 2014/spring 2015/fall 2015/spring 2016) 


\section{University of Louisville, Kent School of Social Work, Guest Lecturer}

Psychosocial Practice in Oncology I: Interpersonal Practice (fall 2014)

\section{University of Louisville, Kent School of Social Work, Teaching Assistant}

Advanced Social Work Practice (fall 2012)

\section{Catholic University of the Sacred Heart, Teaching Assistant}

Families, Stress and Coping (spring 2008)

Health Psychology (fall 2007, fall 2008)

\section{CLINICAL EXPERIENCE}

2010-2011 Massachusetts General Hospital \& Yawkey Center for Outpatient Care. Clinical Social Work Intern

Provided individual counseling to pregnant women in Inpatient and Outpatient settings

Provided couple support and counseling for infertility, prematurity, genetic diagnosis, stillbirth, loss and previous cancer history

Facilitated family support and counseling for high-risk pregnancy, long-term hospitalization, NICU and SCN admission

Provided psychosocial assessments, diagnosis and treatment

2009-2010 Deutsches Altenheim, German Centre for Extended Care, MA. Clinical Social Work Intern

Provided psychosocial assessments, diagnosis, treatment and discharge planning Provided individual counseling to older adults in long term and transitional care

Facilitated support groups for male caregivers of cancer patients

Facilitated family support and counseling for palliative care and death and dying

Developed educational materials about end-of-life decision making for family members

2008-2009 Catholic University of the Sacred Heart, Department of Psychology, Milan.Post-Master Internship

Provided individual and couple therapy to breast cancer patients, partners and caregivers

Managed crisis intervention involving patients, families and health related issues

Implemented psychosocial patient/family care plan through collaboration with several health professionals

Provided psychological support to breast cancer patients during their medical appointments with Massimo Grassi, MD 


\section{PROFESSIONAL SERVICE}

\section{Peer Review}

Reviewer: Journal of Psychosocial Oncology, 2015- present

Reviewer: Psycho-Oncology, 2014-present

Reviewer: Supportive Care in Cancer, 2012-present

Abstract Reviewer: American Psychosocial Oncology Society Annual Conference, 2016

Abstract Reviewer: World Congress of Psycho-Oncology, 2015

Abstract Reviewer: Sixth International Meeting on Stress \& Coping. Couples Coping with Cancer-Related Stress, 2013

\section{To Profession}

Research Committee, American Psychosocial Oncology Society, 2015-present

Mentoring Program Task Force, American Psychosocial Oncology Society, 2014present

Association of Oncology Social Work (AOSW), Annual Meeting of the Social Work Research Group (SWORG) Committee, Louisville, 2011- 2015

Sixth International Meeting on Stress \& Coping. Couples Coping with CancerRelated Stress 2013: Networking events for graduate students

\section{To Community}

Psychosocial Oncology Development Committee, James Graham Brown Cancer Center, 2011- present

Volunteer, Gilda's Club Louisville, 2014-present

Volunteer, S. Leopoldo Mandic Hospital Children's Unit, Merate, 2004-2005

Volunteer, Italian Association for Organ Donation, 2002-present

Volunteer, “S. Luigi” Church Youth Organization, Verderio, 1998-2002

\section{ADDITIONAL TRAINING \& SKILLS}

\section{Research}

2015 Dyadic Data Analysis Workshop; Michigan State University, July 13-17

2014 Hierarchical Linear Modeling: University of Pittsburgh School of Education, May $19-23$

Structural Equation Modeling: Advanced Research Design \& Analysis II (fall 2013); Advanced Statistical Computer Application in Criminal Justice (fall 2014), University of Louisville 
2013 Achieving Better Insights from Psycho-Oncology Research: The Analysis of Longitudinal Data. International Psycho-Oncology Society 15th World Congress 6-8 November, Rotterdam, The Netherlands

2011 Biosocial Methods for Social Work Research. SSWR Summer Institute Workshop, University of Pittsburgh School of Social Work, July 26-28.

Statistical Packages: SPSS, R, LISREL, STATA, MLwiN

\section{Teaching}

2015 Delphi Center for Teaching \& Learning - Reading Circle: Teaching for Critical Thinking: Tools and Techniques to Help Students Question Their Assumptions, October 9-30

i2a Institute, University of Louisville, Delphi Center for Teaching \& Learning, May 18-19

2014 iCOPE Interdisciplinary Curriculum for Oncology Palliative Care Education, September 15

2013 Graduate Teaching Assistant Academy, University of Louisville School of Interdisciplinary and Graduate Studies \& Delphi Center for Teaching \& Learning (fall 2012/spring 2013)

Delphi U: Principles of Online Course Design, University of Louisville Delphi Center for Teaching \& Learning, June 2013

Delphi U Level II, University of Louisville Delphi Center for Teaching \& Learning, June 2013

\section{PROFESSIONAL ASSOCIATIONS}

American Psychosocial Oncology Society (APOS) - Member in Training; since 2012

Association of Oncology Social Work (AOSW) - Student Member, since 2012

International Psycho-Oncology Society (IPOS) - Member in Training; since 2011

Society for Adolescent and Young Adult Oncology (SAYAO) - Charter Member, since 2012

Society for Social Work and Research (SSWR) - Student Member, since 2010

\section{LANGUAGES}

Italian: Native reading, speaking and writing

English: Excellent reading, speaking and writing 